\title{
Novel Micro-Aggregation Techniques for Secure Statistical Databases
}

\author{
By \\ Ebaa Fayyoumi \\ A thesis submitted to \\ the Faculty of Graduate Studies and Research \\ in partial fulfilment of \\ the requirements for the degree of \\ Doctor of Philosophy \\ Ottawa-Carleton Institute for Computer Science \\ School of Computer Science \\ Carleton University \\ Ottawa, Ontario \\ September 2008 \\ (c) Copyright \\ 2008, Ebaa Fayyoumi
}




$\begin{array}{ll}\begin{array}{l}\text { Library and } \\ \text { Archives Canada }\end{array} & \begin{array}{l}\text { Bibliothèque et } \\ \text { Archives Canada }\end{array} \\ \begin{array}{l}\text { Published Heritage } \\ \text { Branch }\end{array} & \begin{array}{l}\text { Direction du } \\ \text { Patrimoine de l'édition }\end{array} \\ \begin{array}{l}\text { 395 Wellington Street } \\ \text { Ottawa ON K1A ON4 } \\ \text { Canada }\end{array} & \begin{array}{l}\text { 395, rue Wellington } \\ \text { Ottawa ON K1A 0N4 } \\ \text { Canada }\end{array}\end{array}$

Yourfile Votre référence

ISBN: 978-0-494-43893-0

Our file Notre référence

ISBN: 978-0-494-43893-0

NOTICE:

The author has granted a nonexclusive license allowing Library and Archives Canada to reproduce, publish, archive, preserve, conserve, communicate to the public by telecommunication or on the Internet, loan, distribute and sell theses worldwide, for commercial or noncommercial purposes, in microform, paper, electronic and/or any other formats.

The author retains copyright ownership and moral rights in this thesis. Neither the thesis nor substantial extracts from it may be printed or otherwise reproduced without the author's permission.
AVIS:

L'auteur a accordé une licence non exclusive permettant à la Bibliothèque et Archives Canada de reproduire, publier, archiver, sauvegarder, conserver, transmettre au public par télécommunication ou par l'Internet, prêter, distribuer et vendre des thèses partout dans le monde, à des fins commerciales ou autres, sur support microforme, papier, électronique et/ou autres formats.

L'auteur conserve la propriété du droit d'auteur et des droits moraux qui protège cette thèse. $\mathrm{Ni}$ la thèse ni des extraits substantiels de celle-ci ne doivent être imprimés ou autrement reproduits sans son autorisation.
In compliance with the Canadian

Privacy Act some supporting forms may have been removed from this thesis.

While these forms may be included in the document page count, their removal does not represent any loss of content from the thesis.
Conformément à la loi canadienne sur la protection de la vie privée, quelques formulaires secondaires ont été enlevés de cette thèse.

Bien que ces formulaires aient inclus dans la pagination, il n'y aura aucun contenu manquant.

\section{Canada}


Dedicated with all my love

to

my loving husband, Ismail,

and to

my wonderful daughter, Zeina.

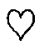


"To be sure, this word information in communication theory relates not so much to what you do say, as to what you could say. That is, information is a measure of one's freedom of choice when one selects a message."

(C. E. SHANNON and W. WEAVER [142]) 


\begin{abstract}
A Micro-Aggregation Technique $(M A T)$ is a Statistical Disclosure Control scheme that is used to protect a $S D B$. The aim of this Doctoral Thesis and our research endeavor is to study the Micro-Aggregation Problem and to design and implement novel $M A T s$ that could prevent the disclosure of confidential information, and simultaneously not significantly harm the utility of the data being provided to the user. The research undertaken enhances the general performance of MATs by either minimizing the computation, or by minimizing the value of the Information Loss $(I L)$, the Disclosure Risk $(D R)$, or a composite measure of the latter two indices, the Scoring Index $(S I)$.
\end{abstract}

This Thesis describes four new methodologies, which are our primary contributions:

1. We have considered an existing $M A T$ algorithm, namely the so-called $k$-Ward algorithm and optimized it for large data sets. This has been done by taking advantage of the distinct properties of the distance matrix and/or utilizing the principle of recursion.

2. We have merged the rich fields of Learning Automata $(L A)$ and $M A T s$ to present a novel Fixed-Structure $L A$ to micro-aggregate a micro-data file. The proposed algorithm has been shown to be superior to the state-of-the-art methods.

3. We have suggested how a neural network philosophy can lead to an enhanced $M A T$. To achieve this we have investigated the effect of replacing the Euclidean distance, which is used to measure the similarity between the individual records in the micro-data file, by the association and the interaction rules that govern the neural network.

4. We have proposed a methodology to use the theory of causal networks and dependency to improve any $M A T$. The results of such a preprocessing phase 
assist in solving a very difficult problem, namely that of determining the number and identity of the variables to be used in any micro-aggregation process.

The Thesis also lists various open problems and avenues for future research. 


\section{Acknowledgements}

This Thesis is the end of my long journey towards obtaining my Ph.D. degree in Computer Science. This could not have happened without the support of many people. The Thesis will not be complete without an acknowledgment of my gratitude.

First, I thank God who helped me through all the tests that came my way during the past five years. You have certainly enriched my life. May your name be exalted, honored, and glorified.

I am deeply indebted to my Supervisor, Professor John Oommen, who traveled with me on this long journey. I could not have wished for a better supervisor. It is difficult to overstate my gratitude to him. He was always there to listen to me, and to offer me sound advice. He taught me that there were different ways to approach a research problem, and instilled in me the persistence needed to accomplish the goal. He has been both a friend and mentor. He taught me how to write academic papers, had confidence in me when I doubted myself, and was able to draw out my research strengths. He was always there to meet me and to discuss my ideas. He was also very through in preparing the corresponding papers and chapters. Without his enthusiasm, his inspiration, and his tireless efforts to explain things clearly and simply, I could have lost my way, and would not have finished this dissertation.

Besides my advisor, I am also grateful to my Thesis Committee for their evaluation and for their constructive comments. I am thankful that in the midst of all their activity, they took time to provide feedback as members of the Committee. I specially thank Dr. Shirley Mills for her friendship and encouragement, and for permitting me to attend her courses on Data Mining and Categorical Data Analysis. I also thank Dr. Herna Viktor and Dr. Anil Maheshwari for their insightful comments.

I am extremely grateful to Dr. Josep Domingo-Ferrer for all his support and advice, and for providing me with the real-life data sets. I am also thankful to him, Dr. Josep Mateo-Sanz, and Dr. Francese Sebe for responding to my e-mails and answering my queries. Without their help, I would have been hindered by many 
hurdles.

I am indebted to Dr. J. P. Corriveau, the Graduate Director in the School of Computer Science, at Carleton University, for looking after the issues that relate to my financial support.

I am grateful to the staff at the School of Computer Science for helping me in numerous ways. In particular, I mention Linda Pfeiffer, Claire Ryan, Sharmila Namasivayampillai, and Gerardo Reynaga. I would also like to thank the members of Dr. Oommen's previous and present research group, with a special reference to Dragos, Khalid, Denis, and my friend, Cesar.

Where would I be without my family? It is hard for me to find words to express my gratitude to my parents, Naser Eddin Fayyoumi and Heyam Saeidan. They gave me life in the first place, raised me with both care and sincerity, and taught me the fundamental values of life itself. Without your gentle love, endless support, and encouragement, this Thesis would not have materialized. My aim is to attain the goals that you set for me: "Be a useful person and contribute to socicty". I would like to thank my sisters Enas, Alaa, Eman, Enal, and Atheel for encouraging me to continue my studies, believing in me, and taking care of our parents, while I was immersed in my research.

My literary skills are far too weak to adequately express my appreciation to my husband, Dr. Ismail Al-Fasfous. His dedication, love, and persistent confidence in me has taken the load off my shoulders. He has always encouraged me to accomplish my tasks in a professional and commendable manner. I appreciate his constant patience, understanding, endless support, and encouragement in every situation. I am honored to know him and humbled to share life with him. His love and kindness are foundation stones for all that I am and currently have. Now that my Thesis is completed, I am looking forward to spending more time with him.

Finally, there is one extraordinary person who deserves a very sincere acknowledgment, which is my daughter, Zeina Al-Fasfous. I owe her so much! She has been my 
source of strength and love. I could not have survived the pressures of life without her. I am glad that she is growing up, and I hope that she will be proud of me when she understands what these last years have entailed. Thank you, pretty girl, for your love, and hugs, and for motivating me to keep reaching for excellence. Thank you for everything that you are, and for everything that you will become.

The financial support for my Doctoral studies was partially provided by The Hashemite University in Zarqa, Jordan. This assistance was crucial to the success of my research, and, I gratefully acknowledge it. I also thank the administration of the Hashemite University for releasing me from my academic responsibilities while I undertook this major endeavor! 


\section{Contents}

1 Introduction 1

1.1 Introduction . . . . . . . . . . . . . . . . . 1

1.2 Motivations and Objectives .................. 5

1.2 .1 Motivations ................. 6

1.2 .2 Objectives ....................... 7

1.3 Organization of the Thesis ................... 9

2 Literature Review $\quad 12$

2.1 Introduction . . . . . . . . . . . . . . . . 12

2.1.1 Application Domain . . . . . . . . . . . . . . 15

2.2 Types of Statistical Data . . . . . . . . . . . . . 16

2.3 Variables Types . . . . . . . . . . . . . . . . 18

2.4 Micro-data Performance Measures . . . . . . . . . . . . . . . . . . . . 21

2.4.1 Micro-data Disclosure Risk . . . . . . . . . . . . . . 21 
2.4 .2 Micro-data Information Loss . . . . . . . . . . . . . 26

2.4 .3 Trade-off between DR and IL . . . . . . . . . . 32

2.5 Micro-data Protection . . . . . . . . . . . . . . 34

2.5.1 Perturbative Masking Methods . . . . . . . . . . . 37

2.5.2 Non-Perturbative Masking Methods . . . . . . . . . . . 42

2.6 Micro-Aggregation Techniques $(M A T s) \ldots \ldots \ldots \ldots$

2.6 .1 Introduction $\ldots \ldots \ldots \ldots \ldots \ldots \ldots \ldots$

2.6.2 Micro-Aggregation Problem $(M A P) \ldots \ldots \ldots 4$

2.6 .3 State-of-Art: MATs . . . . . . . . . . . . . . . . 48

2.6.3.1 Uni-Variate MATs . . . . . . . . . . . . . 49

2.6.3.2 Multi-Variate MATs . . . . . . . . . . . 56

2.6 .4 Categorical MATs . . . . . . . . . . . . . . 65 65

2.6 .5 Additional Applications . . . . . . . . . . . 65

2.6.6 Properties and Characteristics of the MAT $\ldots \ldots \ldots 67$

2.6.6.1 Variants of the Methods . . . . . . . . . . . 67

2.6.6.2 Inference Control in Data Mining Versus MATs . . 71

2.6.6.3 Clustering Techniques Versus MATs . . . . . . 72

3 Enhancing the Heuristic $k$-Ward MAT $\quad 73$

3.1 Introduction . . . . . . . . . . . . . . 73 
3.1.1 Contribution of the Chapter . . . . . . . . . . . 74

3.2 Ward's Method . . . . . . . . . . . . . . . . 76

$3.3 k$-Ward Micro-Aggregation Technique . . . . . . . . . . 78

3.4 Optimized $k$-Ward Micro-aggregation Technique . . . . . . . . 79

3.4.1 Optimizing Distance-Based Computations . . . . . . . . 80

3.4.2 Recursive $k$-Ward Optimization . . . . . . . . . 87

3.5 Experimental Results . . . . . . . . . . . . . . . . 9 91

3.5 .1 Data Sets . . . . . . . . . . . . . . . 9 91

3.5.2 Results for Uni-variate $\mathrm{MATs}_{\mathrm{N}} \ldots \ldots \ldots \ldots$

3.5.3 Dynamic threshold . . . . . . . . . . . . . . . 95

3.5.4 Results for Multi-variate MATs . . . . . . . . . . . . . 98

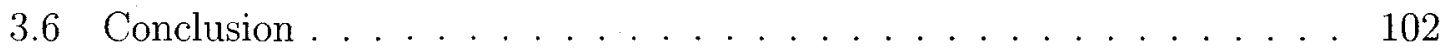

4 A Fixed Structure LA-Based MAT 104

4.1 Introduction . . . . . . . . . . . . . . . . . . . 104

4.1.1 Contribution of the Chapter . . . . . . . . . . . 105

4.2 Learning Automata $(L A) \ldots \ldots \ldots \ldots \ldots \ldots$

4.2.1 Similarities Between the MAP and the EPP . . . . . . 109

4.2 .2 Fundamentals . . . . . . . . . . . . . . . . . 109

4.2 .3 Object Migrating Automaton $(O M A) \ldots \ldots \ldots \ldots$ 
4.2.4 Restrictions of the $O M A$ to the $M A P \ldots \ldots 113$

4.3 Object Migrating Micro-aggregated Automaton $(O M M A) \ldots \ldots \ldots \ldots \ldots$

4.3.1 Formal Properties of The OMMA . . . . . . . . . 128

4.4 Comparing the $M D A V$ and $O M M A$ Methods . . . . . . . . . . 129

4.5 Experimental Results . . . . . . . . . . . . . . . . 134

4.5.1 Data Sets ........................ 134

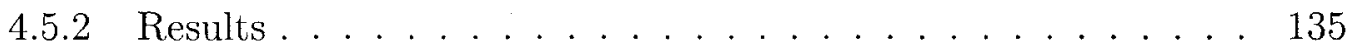

4.6 Conclusions . . . . . . . . . . . . . . . . . . . 149

5 Using Neural Methods to Solve the MAP 150

5.1 Introduction . . . . . . . . . . . . . . . 150

5.1.1 Contribution of the Chapter . . . . . . . . . . . . 151

5.2 Associative Clustering Neural Network $(A C N N)$. . . . . . . . 153

5.3 Interactive-Associative Micro-Aggregation Technique $(I A M A T) \ldots \ldots \ldots \ldots$. . . . . . . . . . . . . . . . . . .

5.3.1 Inadequacy of Using the $A C N N$ Directly . . . . . . . . . 157

5.3.1.1 Feature Values ............... 159

5.3.1.2 Ineffectiveness of Sigmoidal Mappings . . . . . . 159

5.3.1.3 Transitive-Closure-like Properties ... . . . . . . 160

5.3.1.4 One-shot Training . . . . . . . . . . . 161 
5.3 .2 Design of the $I A M A T \ldots \ldots \ldots \ldots 16 \ldots$

5.4 Experimental Results . . . . . . . . . . . . . . . 165

5.4 Data Sets ......................... 165

5.4 Results...................... 166

5.5 Conclusions . . . . . . . . . . . . . . . . 177

6 Utilizing Dependence-Based Information to Enhance MATs 179

6.1 Introduction . . . . . . . . . . . . . . . . 179

6.1.1 Contribution of the Chapter .............. . . 180

6.2 Enhancing Micro-Aggregation with Dependence $(E M A D) \ldots \ldots \ldots \ldots \ldots 2$

6.3 Experimental Results . . . . . . . . . . . . . . . . 191

6.3.1 Data Sets . . . . . . . . . . . . . . . . 191

6.3 .2 Results....................... 193

6.4 Conclusions ......................... 216

7 Conclusion and Future Research $\quad 217$

7.1 Overall Contributions . . . . . . . . . . . . . . . 217

7.2 Enhancing the Heuristic $k$-Ward MAT . . . . . . . . . . 218

7.2 .1 Contributions . . . . . . . . . . . . . 218

7.2 .2 Future work . . . . . . . . . . . . . . . . . 219 
7.3 Using Fixed Structure LA to Solve the MAP . . . . . . . . 220

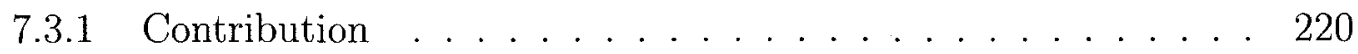

7.3 .2 Future Work . . . . . . . . . . . . . . . . . . . 221

7.4 Using NN Methods to Solve the MAP. . . . . . . . . . . . . 221

7.4 .1 Contribution . . . . . . . . . . . . . . 221

7.4 .2 Future Work . . . . . . . . . . . . . . . . . . 222

7.5 Utilizing Dependence-Based Information for MATs . . . . . . . 223

7.5 .1 Contribution ................... 223

7.5 .2 Future Work . . . . . . . . . . . . . . . . . 223

7.6 Future Research Avenues . . . . . . . . . . . . . . . . . . . . 224

$\begin{array}{ll}\text { Bibliography } & 228\end{array}$ 


\section{List of Tables}

2.1 Perturbative methods for various data types. . . . . . . . .

2.2 Non-Perturbative methods for various data types. . . . . . . . . .

2.3 The classification of the numerical uni-variate micro-aggregation methods. . . . . . . . . . . . . . . . . . . . . 57

2.4 The classification of the numerical multi-variate micro-aggregation meth-

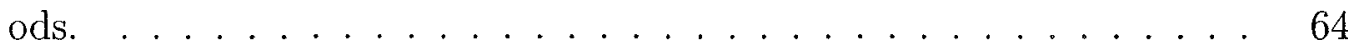

3.1 Statistical Summary for Tarragona data set . . . . . . . . . . . 92

3.2 Statistical Summary for Census data set $\ldots \ldots \ldots \ldots$

3.3 Comparison between the original $k$-Ward_MAT, $k W$, and the optimized $k W D$ on the Tarragona and the Census data sets. In this case we used the value of $k=3$.

3.4 Comparison between the original $k$-Ward_MAT, $k W$, and the optimized $k W R$ using a fixed threshold on the Tarragona data set. In this case we used the value of $k=3 \ldots \ldots \ldots \ldots \ldots$

3.5 Comparison between the original $k$-Ward_MAT, $k W$, and the optimized $k W R$ using a fixed threshold on the Census data set. In this case we used the value of $k=3 \ldots \ldots \ldots \ldots \ldots$ 
3.6 Comparison between the original $k$-Ward_MAT, $k W$, and the optimized $k W R$ using a dynamic threshold on the Tarragona data set. In this case we used the value of $k=3 \ldots \ldots \ldots \ldots$

3.7 Comparison between the original $k$-Ward_MAT, $k W$, and the optimized $k W R$ using a dynamic threshold on the Census data set. In this case we used the value of $k=3 \ldots \ldots \ldots \ldots \ldots \ldots$

3.8 Comparison between the original $k$-Ward_MAT, $k W$, and the optimized $k W D R$ which is a simultaneous combination of both $k W D$ and $k W R$ on the Tarragona data set. In this case we used the value of $k=3$ and a fixed threshold. . . . . . . . . . . . . .

3.9 Comparison between the original $k$-Ward_MAT, $k W$, and the optimized $k W D R$ which is a simultaneous combination of both $k W D$ and $k W R$ on the Census data set. In this case we used the value of $k=3$ and a dynamic threshold . . . . . . . . . . . . . .

3.10 Results for multi-variate $M A T s$ for the Tarragona data set obtained by projecting using the $F P C$ and the $Z S$ indices, In the table we compare the original $k$-Ward_MAT, $k W$, and the optimized $k W D, k W R$ using either a fixed threshold, $(k W R-F)$, or a dynamic threshold, $(k W R-$ $D$ ), and $k W D R$ which is a simultaneous combination of both $k W D$ and $k W R-D$, by setting $k=3 \ldots \ldots \ldots \ldots \ldots$

3.11 Results for multi-variate MATs for the Census data set obtained by projecting using the $F P C$ and the $Z S$ indices, In the table we compare the original $k$-Ward_MAT, $k W$, and the optimized $k W D, k W R$ using either a fixed threshold, $(k W R-F)$, or a dynamic threshold, $(k W R-$ $D)$, and $k W D R$ which is a simultaneous combination of both $k W D$ and $k W R-D$, by setting $k=3$.

4.1 Information loss measures. . . . . . . . . . . . . . . . . . 131 
4.2 Comparison of the percentage of the $I L$ between the $M D A V$ and the $O M M A$ on the Tarragona and Census data sets (uni-variate Methods). In this case the value of $k$ was set to be $k=3$. The column "Converge" indicates the index of the cycle in the learning phase when the value of the total $S S E$ does not change. . . . . . . . . . . . . 136

4.3 Comparison of the percentage of the $I L$ and the computation time between the $M D A V$ and the $O M M A$ on the real-life data sets (Tarragona and Census), and simulated data sets (Uniform and Normal distributions) for multi-variate methods. . . . . . . . . . . . . 137

4.4 Scoring the $M D A V$ and $O M M A$ with respect to the $G_{I L}$ and $G_{D R}$ by computing the index $S I$, for $k=3,4$, and 5 by using Census data set and the simulated Uniform and Normal distributed data sets. . . . .

4.5 Comparison of the percentage of the $I L$ and the computation time between the $M D A V$ and the $O M M A$ on simulated uniform and normal data sets for multi-variate methods. This table investigates the scalability with respect to data size. . . . . . . . . . . . . . . .

4.6 Comparison of the percentage of the $I L$ and the computation time between the $M D A V$ and the $O M M A$ on simulated uniform and normal data sets for multi-variate methods. This table investigates the scalability with respect to the data dimensionality. . . . . . . . . .

4.7 Comparison of the percentage of the $I L$ and the computation time between the $M D A V$ and the $O M M A$ on simulated uniform and normal data sets for multi-variate methods. This table investigates the scalability with respect to the number of records per group. . . . . . . 
5.1 Comparison of the percentage of the $I L$ and the computational time between the MDAV and the IAMAT on the real-life data sets (Tarragona and Census), and simulated data sets (Uniform and Normal distributions) for multi-variate methods. . . . . . . . . . 167

5.2 Scoring the $M D A V$ and $I A M A T$ with respect to the $G_{I L}$ and $G_{D R}$ by computing the index $S I$, for $k=3,4$, and 5 by using the Census data set and the simulated Uniform and Normal distributed data sets. 168

5.3 Comparison of the percentage of the $I L$ and the computation time between the $M D A V$ and the IAMAT on simulated data involving the uniform and normal distributions. The results demonstrate the scalability of the IAMAT with respect to the size of the data set

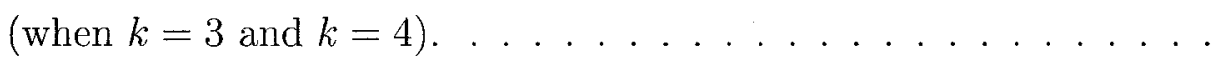

5.4 Comparison of the percentage of the $I L$ and the computation time between the $M D A V$ and the IAMAT on simulated data involving uniform and normal distributions. The results demonstrate the scalability of the IAMAT with respect to the dimensionality of the micro-data file.174

5.5 Comparison of the percentage of the $I L$ and the computation time between the $M D A V$ and the IAMAT on simulated data involving uniform and normal distributions. The results demonstrate the scalability of the IAMAT with respect to the number of records per group. 177

6.1 The characteristics of various data sets. . . . . . . . . . . . 191

6.2 The probability values used in generating the corresponding random variables when the corresponding probabilities for the sibling nodes in the structural dependence tree are related. . . . . . . . . . 202 
6.3 The probability values used in generating the corresponding random variables when the corresponding probabilities for the sibling nodes in the structural dependence tree are unrelated. . . . . . . . . . 202

6.4 The value of the $I L$ obtained by using the $M D A V$ multi-variate $M A T$ after projecting various data sets into the specific number of variables. 206

6.5 The value of the $I L$ obtained by using the $M D A V$ multi-variate $M A T$ after projecting various data sets using 3 variables by using the $E M I M$ metric to calculate the edge weights in the connected undirected graph. 208

6.6 The value of the $I L$ obtained by using the $M D A V$ multi-variate $M A T$ after projecting various data sets using 4 variables by using the $E M I M$ metric to calculate the edge weights in the connected undirected graph. 209

6.7 The value of the $I L$ obtained by using the $M D A V$ multi-variate $M A T$ after projecting various data sets using 3 variables by using the $\chi^{2}$ metric to calculate the edge weights in the connected undirected graph. 212

6.8 The value of the $I L$ obtained by using the $M D A V$ multi-variate $M A T$ after projecting various data sets into 3 variables assuming normality. 214

6.9 Characteristics of the EMIM, $\chi^{2}$ and Correlation metrics in calculating the edges weights of the connected undirected graph. . . . . . 216 


\section{List of Figures}

2.1 Fixed-size groups versus variable-sized groups. . . . . . . . . . . 54

2.2 Grouping with the maximum distance criterion. . . . . . . . .

3.1 Comparison between $k$-Ward_MAT and the Optimized version $k W D$ in computing the distance matrix using the stored matrix approach. In the initialization step, (a) $k$-Ward_MAT computes all values that lie above the diagonal, while (b) $k W D$ computes only the diagonal which is above the main diagonal. At each basic step, (while merging groups $G_{i}$ with $G_{i+1}$, (c) $k$-Ward_MAT computes all values that lie on the column corresponding to $G_{i, i+1}$ and the row $G_{i, i+1}$ above the diagonal, while (d) $k W D$ computes at most two values $\left(G_{i, i+1}, G_{i-1}\right)$

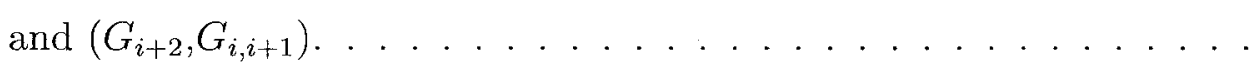

3.2 Components of the similarity distance matrix for a micro-data file that have to be computed for algorithm $k W D \ldots \ldots \ldots \ldots$. . . . .

3.3 Comparison between $k$-Ward_MAT, $k W D, k W R$, and $k W D R$ using the Tarragona Data set. In this case we used the value of $k=3 \ldots 100$

4.1 The $O M M A$ process used to generate the micro-aggregated file. . . 116 
4.2 The automaton has been rewarded, since both $R_{i}$ and $R_{j}$ are similar and located in the same group. . . . . . . . . . . . . . . 121

4.3 The automaton has been penalized, since $R_{i}$ and $R_{j}$ are similar but located in distinct groups. Neither of them is at the boundary state. .

4.4 (A) The automaton has been penalized, since $R_{i}$ and $R_{j}$ are similar but located in distinct groups. $R_{j}$ is at the boundary state, while $R_{i}$ is not at the boundary state. After searching for the most suitable record that leads to the minimum value of the $S S E$, we have two choices for the scenario when $k=3$. In the first case (see (B) above), we perform a physical swap between $\left(R_{u}, R_{j}\right)$. In the second case, (in (C)), no record-swap leads to a smaller value of the $S S E$, and thus, the tuple $<R_{i}, R_{j}>$ is deleted from the similarity list. . . . . . .

4.5 (A) The automaton has been penalized, since $R_{i}$ and $R_{j}$ are similar but located in distinct groups. However, both of them are in a boundary state. After searching for the most suitable record that leads to the minimum value of the $S S E$, we encounter four swapping options for the scenario when $k=3$ - which, in turn, depend on the best candidate record. For example, a physical swap between $\left(R_{i}, R_{x}\right)$ is done when $R_{x}$ is the best candidate (as in case (B)). Similarly, a physical swap between $\left(R_{j}, R_{v}\right)$ is done, when $R_{v}$ is the best candidate, (as in $(\mathrm{C})$ ).

4.6 The effect of invoking the $M D A V$ and $O M M A$ on the $G_{I L}$ and $G_{D R}$ indices when $k=3,4$ and 5 for Census data set. . . . . . . . .

4.7 The improvement of the $O M M A$ in reducing the percentage value of the $I L$ as a function of the cardinality of the data set using uniformly distributed data when $k=3 \ldots \ldots \ldots \ldots$. . . . . . . . 143 
4.8 The improvement of the $O M M A$ in reducing the percentage value of the $I L$ as a function of the cardinality of the data set using normally distributed data when $k=3 \ldots \ldots \ldots \ldots \ldots$. . . . . . . 143

4.9 The improvement of the $O M M A$ in reducing the percentage value of the $I L$ as a function of the dimension of the data set using uniformly distributed data when $k=3$ and $n=3,000 \ldots \ldots \ldots 146$

4.10 The improvement of the $O M M A$ in reducing the percentage value of the $I L$ as a function of the dimension of the data set using normally distributed data when $k=3$ and $n=3,000 \ldots \ldots \ldots 146$

4.11 The improvement of the OMMA in reducing the percentage value of the $I L$ as a function of the number of records per group using uniformly distributed data when $n=2,400$ and $d=16 \ldots \ldots \ldots \ldots$

4.12 The improvement of the $O M M A$ in reducing the percentage value of the $I L$ as a function of the number of records per group using normally distributed data when $n=2,400$ and $d=16 \ldots \ldots \ldots \ldots 147$

5.1 The structure of ACNN $\ldots \ldots \ldots \ldots \ldots \ldots$

5.2 The effect of invoking the $M D A V$ and IAMAT on the $G_{I L}$ and $G_{D R}$ indices when $k=3,4$ and 5 for Census data set. . . . . . . 170

5.3 The improvement of the IAMAT in reducing the percentage value of the $I L$ as a function of the cardinality of the data set using normally distributed data when $k=3 \ldots \ldots \ldots \ldots \ldots$

5.4 The improvement of the IAMAT in reducing the percentage value of the $I L$ as a function of the cardinality of the data set using uniformly distributed data when $k=3 \ldots \ldots \ldots \ldots \ldots$ 
5.5 The improvement of the IAMAT in reducing the percentage value of the $I L$ as a function of the dimension of the data set using normally distributed data when $k=3$ and $n=10,000 \ldots \ldots \ldots \ldots$

5.6 The improvement of the IAMAT in reducing the percentage value of the $I L$ as a function of the dimension of the data set using uniformly distributed data when $k=3$ and $n=10,000 \ldots \ldots \ldots \ldots$

5.7 The improvement of the IAMAT in reducing the percentage value of the $I L$ as a function of the number of records per group using normally distributed data when $n=10,000$ and $d=10 \ldots \ldots 176$

5.8 The improvement of the IAMAT in reducing the percentage value of the $I L$ as a function of the number of records per group using uniformly distributed data when $n=10,000$ and $d=10 \ldots \ldots \ldots$

6.1 Equivalent procedures for finding the Maximum Likelihood Estimate of the tree-based dependence from the samples. . . . . . . . . 186

6.2 The fully-connected undirected graph represents the dependence between six random variables. . . . . . . . . . . . . . 188

6.3 An example of a dependence tree used to micro-aggregate the data file containing 6 variables. . . . . . . . . . . . . . . . 190

6.4 The true structures for the simulated data sets . . . . . . . . . . 191

6.5 The best dependence tree for the simulated data sets obtained by using the $E M I M$ metric. . . . . . . . . . . . . . . . 196

6.6 The "inferred" dependence tree for the Sim_1 binary data set as the number of samples increases. The width parameter was set to 100 . .

6.7 The "inferred" dependence tree for the Sim_2 binary data set as the number of samples increases. The width parameter was set to 100 . . 
6.8 The "inferred" dependence tree for the Sim_3 binary data set as the number of samples increases. The width parameter was set to 100. .

6.9 The best dependence tree for the Tarragona data Set obtained by using the EMIM metric with various values of the width parameter. . . . 200

6.10 The best dependence tree for the Census data set obtained by using the EMIM metric with various values of the width parameter. . . . 200

6.11 The best dependence tree for a binomial data sets with 5,000 records and 6 variables. . . . . . . . . . . . . . . . . . . . . 203

6.12 The "inferred" dependence tree for the binary data set as the number of samples increases. In this case, the probabilities between the sibling random variables are related. . . . . . . . . . . . . . . . 203

6.13 The "inferred" dependence tree for the binary data set as the number of samples increases. In this case, the probabilities between the sibling random variables are unrelated. . . . . . . . . . . . . . 203

6.14 The convergence of the corresponding metric for the Set-Up4 data sets by using (a) the EMIM metric to calculate the edges weights. In (a) the probabilities between the siblings are related, and in (b) these probabilities between the siblings are unrelated. . . . . . . . 204

6.15 The best dependence tree for the Tarragona data set obtained by using the $\chi^{2}$ metric with various values of the width parameter. . . . . . . 211

6.16 The best dependence tree for the Census data set obtained by using the $\chi^{2}$ metric with various values of the width parameter. . . . . . .

6.17 The best dependence tree for the real and simulated data sets assuming normality. . . . . . . . . . . . . . . . . . . . 215 


\section{List of Algorithms}

1 Ward Method $[139] \ldots \ldots \ldots \ldots \ldots \ldots$

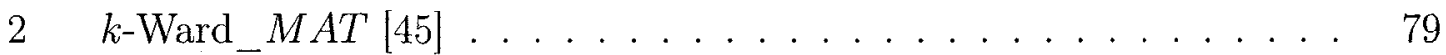

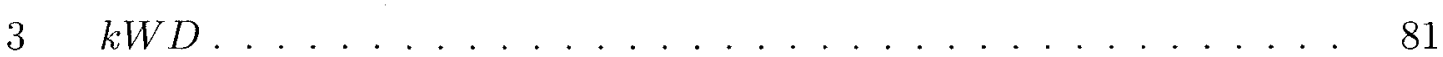

$4 \quad k W R \ldots \ldots \ldots \ldots \ldots \ldots \ldots \ldots \ldots \ldots \ldots$

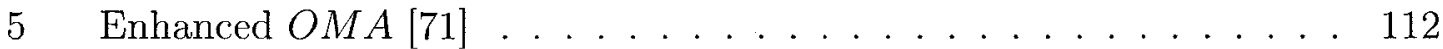

6 Learning Phase in the $O M M A \ldots \ldots \ldots \ldots . \ldots \ldots$

7 Procedure Reward Record . . . . . . . . . . . . . . . . . . . . 123

8 Procedure Penalize_Two_Unbound_Records . . . . . . . . . . . . 123

9 Procedure Penalize_Bound_Unbound_Records . . . . . . . . . . . 124

10 Procedure Penalize_Two_Bound_Records . . . . . . . . . . . . 126

11 Associative Cluster Neural Network (ACNN) . . . . . . . . . . . 158

12 Interactive-Associative Micro-Aggregation Technique (IAMAT) . . 163

$13 E M A D \ldots \ldots \ldots \ldots \ldots \ldots \ldots \ldots \ldots$ 


\title{
List of Abbreviations
}

\author{
ACA : Adaptive Clustering Algorithm \\ $A C N N \quad$ : Associative Cluster Neural Network \\ AI : Artificial Intelligent \\ ASR : Association Similarity Rule \\ DR : Disclosure Risk \\ $E M A D$ : Enhancing Micro-Aggregation with Dependence \\ EMIM : Expected Mutual Information Measure \\ EPP : Equi-Partitioning Problem \\ FSSA : Fixed Structure Stochastic Automata \\ $G_{D R} \quad$ : The overall Global DR \\ $G_{I L} \quad:$ The overall Global IL \\ IAMAT : Interactive-Associative Micro-Aggregation Technique \\ ID : Interval Disclosure \\ IL : Information Loss \\ $k W \quad: k$-Ward MAT \\ $k W D \quad: \quad k$-Ward Diagonal \\ $k W D R \quad$ : Recursive $k$-Ward Diagonal \\ $k W R \quad:$ Recursive $k$-Ward \\ $k W R-D$ : Recursive $k$-Ward Dynamic threshold \\ $k W R-F \quad$ : Recursive $k$-Ward Fixed threshold \\ $L A \quad$ : Learning Automata
}




$\begin{array}{ll}M A P & : \text { Micro-Aggregation Problem } \\ M A T & : \text { Micro-Aggregation Technique } \\ M D & : \text { Maximum-Distance } \\ M D A V & : \text { Maximum Distance Average Vector } \\ M L & : \text { Maximum Likelihood } \\ M S T & : \text { Maximum Spanning Tree } \\ N N & : \text { Neural Network } \\ O M A & : \text { Object Migrating Automaton } \\ O M M A & : \text { Object Migrating Micro-aggregated Automaton } \\ P C A & : \text { Principal Component Analysis } \\ P R A M & : \text { Post-RAndomization Method } \\ R L D & : \text { Record Linkage Disclosure } \\ S D B & : \text { Statistical DataBases } \\ S D C & : \text { Statistical Disclosure Control } \\ S I & : \text { Score Index } \\ S S A & : \text { Sum of Squares Among the groups } \\ S S E & : \text { Sum of Squares Error } \\ S S T & : \text { Total Sum of Squares error } \\ V-M D A V & : \text { Variable-size Maximum Distance to Average Vector } \\ V S S A & : \text { Variable Structure Stochastic Automata } \\ Z S & : \text { Z-Scores }\end{array}$




\section{Chapter 1}

\section{Introduction}

\subsection{Introduction}

The development of empirical research and the growing capacity of modern computer systems have led to Statistical DataBases ( $S D B s$ ) that contain a large amount of information, some of which may be sensitive. $S D B s$ are used to produce statistical summaries about certain groups, such as the sum, count, average, min and max [30]. However, it is essential that these statistical summaries do not disclose the content of any single individual record. This requirement is often difficult to satisfy, because the user may ask many different "legal" queries and deduce confidential information from the responses obtained ${ }^{1}$. For example, comparing the mean salary of two groups differing only by a single record will compromise the salary value of the individual whose record is in one group but not in the other $[2,4,12,30,69,80,86,123]$.

The problem of enhancing the security in $S D B s$ has received a lot of attention in

\footnotetext{
${ }^{1} S D B s$ can be exactly or partially compromised if there is an authorized user who constructs one or more queries using a priori information, which, in turn, can be used to infer the value of a certain field. The most effective methods for compromising $S D B s$ are listed as follows: Generating a query set whose cardinality is unity, using an individual tracker, utilizing a general tracker, or presenting queries that possess the "overlap" property. More details of these mechanisms are given in $|12,30,69|$.
} 
recent years. This is because of the fact that government agencies and private organizations increasingly face the problem of protecting confidential information contained in their databases. Census bureaus have successfully dealt with this problem by removing information from the databases that can easily identify an individual, i.e. Social Insurance Number. They release statistical summaries drawn from a small sample of the entire population $[35,101,97]$.

Generally speaking, the problem at hand concentrates on guaranteeing the security of $S D B s$, and on obtaining useful, unbiased statistical information. Several avenues to achieve this have been reported in the literature, each of them having their own properties.

One approach to solve this problem is to restrict access to the data. INGRES [69], for example, distinguishes between two types of statistical queries: Those that apply to the whole relation and those that apply to a subset of any relation. The first type of query is equivalent to one with a non-characteristic formula, which will be answered normally. As opposed to this, the second type of query is modified with relevant access rules in such a way that a potential intruder only gets Read Access to a subset of rows in the relation and, thus, obtains an answer which approximates the true one. This, of-course, prevents compromising the database, but it is very restrictive for most situations.

Another approach to resolve this is to provide the system with a wide range of powerful techniques, each of which yields inference protection against intruders. The families of these techniques are listed and classified in the literature [16, 86], and a more detailed survey is found in Chapter 2. However, it is generally assumed that they can be classified into three groups: Query set restriction, various perturbationbased approaches, and conceptual schema approaches. These are briefly detailed in the following paragraphs.

\section{Query Set Restriction Approaches}

These techniques protect against inference by restricting and not releasing the 
statistical summaries that could reveal pieces of confidential information about single individuals in the $S D B$. The set of released data is a subset of the set which satisfies the restrictions that have been applied on the size, type of the query result and/or the percentage of the overlap between the result of the current query and the previously submitted queries. Although this approach prevents an exact compromise, it eliminates the usefulness of SDBs. Query set size control, Query set overlap control Auditing, Partitioning and Cell suppression are examples of methods within this category $[16,69]$.

\section{Perturbation-Based Approaches}

These techniques provide inference protection by introducing a modification to the information in an unpredictable way. The information can be modified before answering the queries or after processing them, but rather, before releasing the result to the user. Data perturbation is the name of methods in the first scenario where a proxy database is created from the original database and all the statistical queries are calculated against the proxy database. Methods within the second sub-class are called output perturbation methods, and they do not need a proxy database because all the statistical queries are calculated against the real database, after which noise is added to the result. Data perturbation avoids the inference and always provides consistent results. On the contrary, output perturbation could provide the user with inconsistent results. Data swapping, Fixed data perturbation, Micro-aggregation, Random sample queries, Varying output perturbing, and Rounding are examples of methods within this category $[16,69]$.

\section{Conceptual Approaches}

Methods of this sub-class address the inference problem by altering the conceptual level schema on which the $S D B$ is built. It concentrates on re-designing the schema of the relations. Although the benefit of such solutions are significant with respect to security, the implementation is very expensive because these approaches require major changes to the structure of the database model, as well as the additional time required for uploading sessions to populate the 
tables. The Conceptual Model and Lattice Model techniques are examples of methods within this category $[16,69]$.

The quality of the information released to the user in the presence of an inference protection technique is measured using different criteria such as security, robustness, bias, precision, and consistency $[2,16]$. Security is the fundamental measurement of performance that avoids both partial and exact compromises. Robustness concerns the assumptions made by the system about the supplementary knowledge of the $S D B s$. As opposed to these, the difference between bias and precision is that the bias represents the variance between the perturbed value returned to the user and the true value, while precision refers to the variance in a system's bias, which must be within a specified confidence interval. Finally, consistency represents the ability of a perturbation-based system to respond to the queries without contradiction ${ }^{2}$ or paradox ${ }^{3}$.

As previously mentioned, many researchers have proposed an ensemble of methods for preventing a breach of the security in $S D B s$, while others have constructed models for studying this problem. The research within the scope of this Doctoral Thesis deals with the Micro-Aggregation Technique (MAT) which is considered as one of the perturbative methods. In spite of a few reported weakness described later in the Thesis, MATs have recently emerged as being some of the most promising protection methods [100]. It is used to protect micro-data files by storing the individual records in groups possessing a minimum size constraint. Whenever the query is submitted, it is addressed to a group containing the record, but never to a specific record within the group. This prevents a user from isolating a record with overlapping queries. An $M A T$ possesses many attractive features such as its robust performance, its consistent responses, and ease of implementation. It is important to mention that the $M A T$ offers more protection to outliers than other SDC techniques, and the choice of its unique parameter (which we shall specify later) is less influential than others. The

\footnotetext{
${ }^{2} \mathrm{~A}$ contradiction can occur when the query responses are perturbed. In such a case, there is a possibility that comparing responses of two queries will reveal arithmetic inconsistencies.

${ }^{3} \mathrm{~A}$ paradox refers to responses that violate the properties of statistical queries.
} 
clear connection between the properties of an $M A T$ and the anonymization of SDBs increases the importance of the former $[40,53,57,94,131,133,134]$.

The primary goal of this Thesis is to report a set of newly proposed strategies that enhance the general performance of MATs. These enhancements are obtained by either minimizing the required computational time to micro-aggregate all the microunits in the micro-data file, or by minimizing the value of the Information Loss, $(I L)$, the Disclosure Risk, $(D R)$, or a criterion which is a composite index based on the $I L$ and $D R$. Our research will involve concentrating on all these aspects from a variety of perspectives.

This chapter provides an introduction to this Doctoral Thesis, and illustrates its main motivations and objectives. The chapter is organized as follows: Section 1.2 presents the main motivations and objectives of the work. Section 1.3 gives the organization of the Thesis, and catalogues the main contributions of each chapter.

\subsection{Motivations and Objectives}

As mentioned earlier, this Thesis focuses on MATs that are a family of techniques that modify the micro-data file in order to guarantee the factual anonymity of the data. At the same time, the intention is that there should not be a huge reduction in the information content of the data so that the user is able to get useful, un-biased statistical summaries. Micro-aggregation is a clustering problem with cardinality constraints that originated in the area of Statistical Disclosure Control $(S D C)$ for micro-data. In other words, an $M A T$ is typically implemented by clustering the micro-individual records into groups (where each group satisfies certain group-size constraints) based on the similarity between them, and then replaces the individual values by the aggregated value. 
There are numerous micro-aggregation algorithms; they can be classified as follows: uni-variate vs. multi-variate, heuristic vs. optimal, and fixed-size vs. dataoriented. A detailed description and survey of these methods can be found in Chapter 2. In this Thesis, our aim will be to enhance existing algorithms that belong to the heuristic, uni-variate, data-oriented type, and to develop new algorithms that belong to multi-variate, heuristic, with fixed-size or data-oriented type. The reason for seeking such solutions will be explained presently, and in more detail in Chapter 2. In this regard we mention that although an optimal algorithm for the uni-variate case was proposed in [74], unfortunately, for the multi-variate case, the problem is NP-hard [102, 103].

The main advantage of having several heuristic algorithms with fixed or dataoriented group size constraints is that there are some situations in which the microaggregation of key attributes is insufficient to preserve confidentiality. Therefore, re-computing the micro-aggregated sets using other heuristic algorithms or changing a security parameter (such as the group size constraint), could also lead to potentially superior solutions [42].

\subsubsection{Motivations}

A significant amount of work has been done to devise and optimize $M A T s$, most of which involve invoking clustering as a foundational tool. The methods available for clustering data are numerous and a list would probably number in the hundreds. Rather than re-visit the problem by merely considering new clustering methods, our aim is to see if we can optimize MATs by utilizing some fundamentally new tools. In order to motivate the Thesis, we list these below:

- We would like to see if we can incorporate a recursive strategy in developing MATs. To the best of our knowledge, we are not aware of MATs that have been developed for large data sets, and that are entirely constructed by first invoking $M A T s$ on small-size data sets whose results are subsequently merged 
to yield the final comprehensive result.

- A second motivation for this Thesis is to see if we can devise a set of algorithms for the Micro-Aggregation problem (MAP) that use Learning Automata, $(L A)$. As far as we know, there is no existing $M A T$ for which the basic computational tool is a fixed or variable structure $L A$. Indeed, our aim is to find a strategy by which we can merge the rich fields of $M A T$ and $L A$.

- The third motivation focuses on devising multi-variate $M A T s$ that are based on a neural-based clustering method. Indeed, we are not aware of any Neural Network $(N N)$ technique that has been explicitly used as a fundamental tool to solve the MAP. We would like to investigate whether the concept of homogeneity (similarity) between micro-records can be measured by incorporating the phenomena of Association and the Interaction. Again, to the best of our knowledge, no MAT has been described so as to utilize these criteria.

- The last motivation of this Thesis is to investigate whether it is possible to introduce into the existing $M A T$ solutions, schemes that determine the dependency between the random variables (rather than the records). As far as we know, dependence information has not been used in devising $M A T s$, and it is our hope that our research will lead to methods that utilize such strategies.

This summarizes the motivation for our Thesis.

\subsubsection{Objectives}

With the motivations previously listed serving as a beacon to develop new MATs, the objectives of the Thesis are the following:

1. First of all, we would like to develop fast recursive mechanisms that invoke MATs for large-size data sets. In particular, we would like to optimize the $k$ Ward micro-aggregation algorithm with respect to the computational time for 
both uni-variate and projected multi-variate micro-data sets. Our intention is to increase the speed of the micro-aggregation without sacrificing the ultimate utility of the data. We would like to demonstrate that it is possible to take advantage of the distinct properties of the distance matrix and the principle of recursion, to substantially reduce the effective computational burden of the scheme.

2. We would like to demonstrate how the principles behind the families of fixedstructure stochastic $L A$ can be used to enhance MATs. Integrating these families would lead to novel contributions in both these domains. The results we present prove that this can be achieved for uni-variate and multi-variate microdata sets for fixed-size constraints.

3. We would like to use $N N$ techniques, such as those previously used for associative clustering, to enhance $M A T s$. In particular, our objective is to demonstrate the applicability of the association similarity rule (instead of the Euclidean distance) to yield micro-aggregation. In this regard, we have utilized the Associative Clustering Neural Network learning scheme to effectively solve the MAP.

4. We would like to demonstrate that while developing MATs, it is expedient to incorporate information about the structure and dependency between the random variables in the micro-data file. We have demonstrated that including such information will enhance the process of determining how many variables and which variables should be used in the micro-aggregation process. The question of knowing the most suitable metric (with respect to the required computational time and the accuracy) for estimating the dependence model has also been addressed. 


\subsection{Organization of the Thesis}

In this section, we present the overall organization of the Doctoral Thesis and the content/contributions of each chapter.

\section{- Chapter 2: Literature Review}

This chapter presents the background material needed for the Thesis. We first introduce the concept of $S D C$, and then describe its application domain and the various data types that are currently used in $S D B s$. We then proceed to focus our scope by concentrating on the family of micro-data types in $S D B s$. At this point, we emphasize the importance of the $I L$ and $D R$ measures, and survey the various ways of resolving the conflicting goals that they represent. Additionally, we also summarize the perturbative and non-perturbative $S D C$ methods for micro-data protection. Since our research focuses on the $M A T$, we formally state the $M A P$ and attempt to survey it in a comprehensive manner. Indeed we believe that this chapter represents a complete overview of the stateof-the-art techniques.

\section{- Chapter 3: Enhancing the Heuristic $k$-Ward MAT}

This chapter shows how we can optimize the $k$-Ward method by applying some matrix-based and recursive optimization techniques, thus rendering the $k$-Ward method to be pragmatic. These optimizations involve (a) minimizing the computations done in evaluating the between-class distance matrix, and (b) recursively partitioning the data set before invoking a $k$-Ward strategy, thus enforcing that the latter is invoked on "primitive" small-size sub-groups that terminate the recursion. The proposed optimized versions have been rigorously tested on two reference data sets, and the results have been compared with the standard benchmark methods [45]. The main contribution of this chapter is that we have clearly demonstrated that our new optimized versions minimize the computational time required to micro-aggregate all the individual micro-units in the micro-data file, rendering it a viable option. The computational advantage sometimes exceeds $80 \%$ if one of the optimization approaches is applied by itself, 
and more than $90 \%$ if both the above enhancements are invoked simultaneously. The various optimization strategies are applied to uni-variate data sets, and also on multi-variate data sets projected using the first principle component or the sum of $Z$-scores. The work done in this chapter was published in [65].

\section{- Chapter 4: Using Fixed Structure LA to Solve the MAP}

This chapter presents an interesting new method for a fixed-size, multi-variate, micro-aggregation in the Euclidean space. As mentioned earlier, the very concept of using $L A$ for micro-aggregation is a novel contribution to the $S D C$ field. The method is based on the concept of specifying various levels of uncertainties for pairs of individuals belonging to the same group. To accomplish this, our new $L A$, the Object Migrating Micro-aggregation Automaton (OMMA), increases or decreases these uncertainty levels depending on whether a known similar pair is "correctly" or "incorrectly" clustered by the algorithm. The experimental results presented in this chapter show that this technique is a promising scheme for the $M A P$, since the improvement in the data utility is as high as $10 \%$ and $8 \%$ when compared to the Maximum Distance Average Vector $(M D A V)$ and Maximum Spanning Tree $(M S T)$ schemes, respectively. Besides this, our solution also offers a minimum score value when compared to the $M D A V$ method by using a score index $(S I)$, which is a composite measure incorporating both the $I L$ and the $D R$. The work done in this chapter was published in [64].

\section{- Chapter 5: Using NN Methods to Solve the MAP}

This chapter presents a new practical method, based on the theory of $N N s$, to solve the MAP. The novelty of this chapter is its concentration on introducing a new metric for measuring the similarity. This new metric preserves the meaning of the closeness between the records so as to coalesce them into groups, and to also maximize the actual Interaction among the records inside each group. In general, incorporating the concepts of the Interaction among the records and their mutual Association, has been shown to be advantageous to solve the MAP because they have the effect of minimizing the $I L$. The experimental results presented in this chapter show that this technique is a promising scheme for the 
$M A P$, since the improvement in the data utility compared to the $M D A V$ is as high as $8 \%$ and $14 \%$ for real and simulated data sets, respectively. Again, the method also yields a superior $S I$ value when compared to the $M D A V$. The work done in this chapter was published in [107].

\section{- Chapter 6: Utilizing Dependence-Based Information for MATs}

This chapter shows how the presence and structure of dependency between a set of random variables in the micro-data file is valuable information that can be used as a fundamental indicator before invoking any $M A T$. We present a new automated scheme as a pre-processing stage to determine the number and the identity of the variables that are to be used in the micro-aggregation process. This is achieved by constructing a completely connected undirected graph whose nodes represent the random variables in the micro-data file, whose edges represent the statistically dependencies, and whose edge weights are computed by using either an information theoretic, $\chi^{2}$, or correlation-based measured. The advantages and disadvantages of these respective measures have been investigated. The experimental results presented in this chapter show that such a methodology involving projecting the multi-variate data sets reduces the solution space, which further directly reduces the computation time required to search the entire space combinatorially. Additionally, this methodology leads to a solution whose $I L$ values are close to the minimum value of the $I L$ obtained by exhaustively searching over the entire search space. The work done in this chapter was published in $[106,108]$.

\section{- Chapter 7: Conclusion and Future Research}

This chapter summarizes the work done in the Thesis, gives the final conclusions, and presents suggestions for future research that can be pursued in the area of $S D C$, and in particular, to the $M A P$. 


\section{Chapter 2}

\section{Literature Review}

\subsection{Introduction}

A lot of attention has recently been dedicated to the problem of maintaining the confidentiality of statistical databases through the application of statistical tools, so as to limit the identification of information on individuals and enterprises and simultaneously maximize the data utility. Statistical Disclosure Control/Limitation, $(S D C)$, is a field in statistics and computer science (and in particular, in data mining) that has attracted much attention in recent years. Decision-makers are increasingly demanding more detailed statistical information. Indeed, investigators at universities and research centers have the capacity to perform complex statistical analysis with information obtained from various sources of micro-data. Clearly, the requirement that statistical offices publish more detailed information is growing.

There is, however, another side to this extensive use of data. Inasmuch as more confidential data is at risk, statistical offices are required by law or by established policies to protect the confidentiality of information provided to them by the respondents. This confidentiality is also vital when it concerns guaranteeing the future co-operation of respondents. 
Generally speaking, two broad approaches are used to preserve the confidentiality of statistical information $[2,12,36,37,69,30,142]$ :

- Access Control. Disclosure can be controlled by restricting the access to data in different ways. In this case, access may be granted only to well-defined group members, subject to well-defined conditions and/or in well-defined secure locations.

- $S D C$ Techniques. The released data may be modified to reduce the risk of disclosure.

It is important to mention here that these two approaches are complementary, and are often utilized simultaneously. An agency might choose to release the data either with little modification and under very strict access arrangements, or with considerable modification and subject to much looser arrangements.

The main responsibility of statistical offices is to produce and disseminate pieces of statistical information in a form that is suitable to the policy makers, researchers and the general public. The information released using an $S D C$ scheme may have some degree of data modification, where the modifications fall within a range of two extreme models [20, 53]:

- Data Encryption. If the released data is encrypted, it can be protected very securely. However, if the user is not able to decrypt it, the released data is, obviously, useless $[37,69,81,89,30]$.

- No Modification. If the original data is released without modification, it is clear that its usefulness/accuracy is maximal. But in this case, the confidentiality is not protected against any type of disclosure $[2,37,69,89]$.

The important question that thus arises is one of knowing how the information available can be perturbed or modificd in such a way that the released data can be 
statistically useful, and simultaneously not risk the privacy of the entities involved. Thus, the main goal of SDC is to modify the original data so that it can be "published" without compromising the inherent confidentiality contained in the information. The challenge for an $S D C$ scheme is to achieve this modification with a minimum loss of the details and accuracy sought by the user of the database. Generally speaking, every $S D C$ method has two conflicting goals $[2,102,141,142]$ :

1. Minimize Disclosure Risk $(D R)$ : This index is the risk to the confidentiality of the respondents that the data releaser (typically a statistical agency) would experience as a consequence of releasing the data.

2. Minimize Information Loss $(I L)$ : This index quantifies the value of the released data to legitimate data users.

Clearly, the ultimate goal of $S D C$ techniques lies not only in reducing $D R$, the disclosure risk, but also in increasing the utility of the data to the user. Thus, although deleting (or hiding) all the data and releasing no information would eliminate any statistical disclosure, clearly, such a solution is inadequate, because the analytic value of the data will be absent, and vice versa. The conflict between these two criteria is obvious, and our goal is to optimize them, so as to reach a happy medium or an equilibrium point.

A formal optimization specification for the $S D C$ can be stated in terms of Problem $S D C$ as follows [81, 142].

Problem SDC: An optimal $S D C$ solution aims to: Minimize IL[ $\int$ (Data) $]$, subject to DR $\left[\int(\right.$ Data $\left.)\right]<\epsilon$, where:

Data : The original data.

$\int$ (Data) : The released data after the use of the SDC techniques represented by $\int$. 


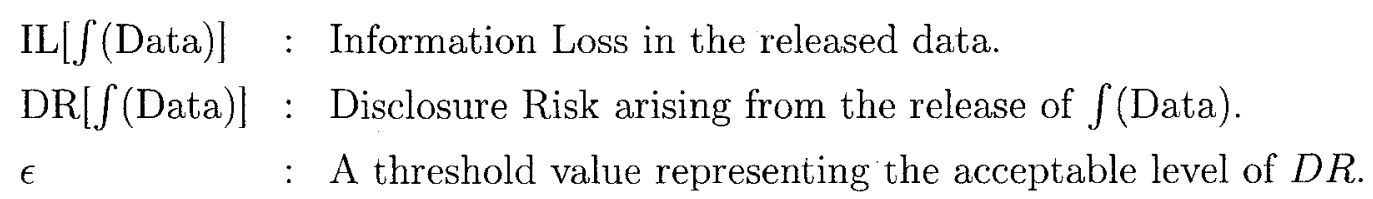

It should be emphasized that, in many applications, it is very difficult (and sometimes impossible) to obtain suitable overall measures for $D R$ and $I L$ that are useful to achieve a formal optimization. This arises not only from the complexity of the problem itself, but also because of the difficulty in making assumptions about the nature of the disclosure threats and the user's information requirements. Additionally, it is clear that the decision about what data can be released depends also on the subjective experience and personal judgments related to the nature of the data [142].

\subsubsection{Application Domain}

There are several areas of application for $S D C$ techniques. These include (but are not limited to) the following $[5,20]$ :

\section{- Official Statistics}

Most countries are legislated by law to compel statistical agencies to guarantee the confidentiality of statistical information whenever they release data collected from citizens or companies. This has been the rationale for enhanced research in $S D C$ in many countries, including those from the European Union (i.e., the $C A S C$ project) and the $U S A$.

\section{- Health Information}

This is one of the most sensitive and important areas demanding privacy. For example, in the USA and in most western countries, privacy restrictions of health-related organizations require the strict and direct regulation of protected health information for use in medical research. 
- E-commerce

Electronic commerce transactions automatically lead to the automated collection of large amounts of consumer data. This information, which can give a competitive edge, is very useful to companies who receive it. Again, it is imperative that such information transfer should not result in the public profiling of individuals. It is, thus, subject to regulation.

\subsection{Types of Statistical Data}

The state-of-the-art in $S D C$ has evolved to such a level that, at the present, it has spawned studies in involving at least three sub-disciplines ${ }^{1}$ :

- Tabular Data

Historically, tabular data are the most common types of data sorted in official databases. Tabular data can be obtained from micro-data by a process called static "aggregation" $[28,102,142]$. The two formats commonly used to display aggregated data $[5,20,102]$ are:

- Frequency tables. These count the number of respondents with specific characteristics.

- Magnitude tables. These are analogous to frequency tables in that they are defined by cross-classification of categorical variables. However, the cells contain aggregate values, of some quantity of interest, for the corresponding respondents.

Tabular data protection is the oldest and most established field within SDC. The goal here is to publish tables in such a way that no confidential information on specific individuals (whose information is present in the tables) can be

\footnotetext{
${ }^{1}$ Distinctions are often made between the ways in which data is released and the "technical" details of the data types. Thus, the proposed methodological approaches differ based on the type of the data involved $[5,89]$.
} 
inferred. In other words, the tables have to be protected against the exact disclosure of an individual attribute ${ }^{2}$, especially when the cells of the table contain information on only one or a very few respondents. A relevant software package in this case is $\tau$-ARGUS [78], and more specific information on the latter can be found in $[101]$.

\section{- Dynamic Databases}

In the case of dynamic databases, the user submits statistical queries (i.e., queries about the average, median) to the database. The most challenging issue for dynamic statistical databases is that the aggregated information obtained by an attacker as a result of successive continuous queries should not permit him to infer any confidential information on specific individuals $[69,102,30]$. One possible strategy is to perturb the answers to the queries $[35,80]$. However, if the perturbed results are not acceptable and exact answers are required, the system refuses to respond to these queries. A second solution, based on query restriction, can be found in [2]. Finally, a third strategy is to provide the user with an accurate interval answer $[5,20]$.

- Micro-data

Data stored and provided in tables do not provide a particularly wide scope for further statistical analysis and investigation. Formally, it is only possible to perform analysis for summaries containing the pertinent information. Consequently, there is considerable demand from researchers for the release of individual data records. It is, thus, only a more recent phenomenon that data collectors have been persuaded to publish micro-data. Therefore, the protection of micro-data is the "youngest" subdiscipline, and this field is experiencing a continuous evolution $[5,20,28,102]$.

Micro-data consist of a series of records, each containing information on an individual unit such as a person, a firm, or an institution. Micro-data in their

\footnotetext{
${ }^{2}$ Three common strategies used to protect against the exact disclosure for tabular data are the socalled "table redesign" scheme [141], the scheme which involves the suppression of individual values [141], and the computationally efficient method involving resampling [38, 142].
} 
simplest form may be represented as a single data matrix, where a row corresponds to the units and the columns to the variables [142].

When it concerns the protection of micro-data files, one has to take into consideration the fact that the information is typically obtained from surveys involving social and business data [67,72]. In both cases, micro-data are released based on the appropriate rules of research, which is, in turn, based on the re-identification risk for the particular micro-data set. In summary, these rules determine how much the information content of a micro-data file has to be reduced or modified so as to be considered safe [101]. For social surveys, these kinds of modifications belong to the class known as "non-perturbative" DSC measures; for the business micro-data they belong to "perturbative" $D S C$ measures $^{3}$ [72].

Generally speaking, there is a dependency between the variables or records. Such dependencies can be of a logical nature or a statistical nature. In this research, we will not take into account such dependency issues, even though sometimes ignoring such dependencies may lead to an underestimation of the disclosure risk. For example, in a record with "Disease = Cancer of the Womb" and "Sex = missing ", it is clear that the attribute for gender satisfies "Sex = Female".

The software package $\mu$-ARGUS can be used to protect micro-data by offering several approaches, as given in [77]. This research document, in subsequent section, will cover the current state-of the art when it concerns the use of $S D C$ methods for micro-data.

\subsection{Variables Types}

As mentioned in Section 2.2, this study focuses on processing micro-data. A microdata set can be viewed as a file with $n$ records or data vectors, each containing

\footnotetext{
${ }^{3}$ Applying non-perturbative techniques on business data tends to yield either an unacceptably high disclosure risk associated with the released data, or a large reduction in the information that renders the analysis meaningless or impossible [72].
} 
data about individuals or respondents, who, in turn, can be persons, companies, etc. Each individual is assigned a data vector formed by a number of variables/attributes $[45,60,142]$.

A variable is an object that is associated with a set of values called a domain. Sometimes, a variable in a data set corresponds to the response to a question in a survey. Some variables are, in turn, derived from other variables through a computational mechanism. Variables play an important role in $S D C$ analysis and in the kind of disclosure control techniques that they admit [142]. Variables in the original unprotected micro-data file can be classified in six categories (which are not necessarily disjoint) $[45,60,142]$ :

- Categorical Variable

A categorical variable is a variable which can take values over a finite set, and for which standard arithmetic operations are undefined. Two main types of categorical attributes can be distinguished [60]:

- Ordinal

An ordinal variable takes values in an ordered range of categories. Thus, global relational operators, such as max and min operations are meaningful, and can be used by $S D C$ techniques. Weekdays, Months, etc, are examples of ordinal variables.

- Nominal

A nominal variable takes values in an unordered range of categories. The only meaningful operator in this case is the comparison of Equality. The variables Eye-Color and Sex are examples of nominal attributes.

In some cases, an additional structure on the domain of the categorical variable can be imposed. Categories sometimes have a hierarchical structure, thus leading to so-called hierarchical variables. Such a hierarchical variable has several partitions defined in its domain. Usually, the least detailed partition is at the highest level, and the most detailed one is at the lowest level. All the remaining 
partitions are at levels between these two extremes [142].

Generally speaking, categorical variables play an important role in identifying the corresponding respondent in the micro-data files [142].

- Continuous Variable

A variable is considered continuous if it is numerical (and real-valued) and if it permits arithmetic operations. When we design methods to protect continuous data, it is imperative that we optimize the use of these arithmetic operations. But, simultaneously, we have to be aware of every combination of numerical values in the original data set to preserve its uniqueness, i.e., if it, indeed, is to be unique. Salary and Age are examples of continuous variables [60].

- Identifying or Key Variable

An identifying or key variable is an attribute that unambiguously identifies the respondent. Examples of such variables are the Social_Security_Number, Passport_Number, Employee_Number, etc. Clearly, all these key variables should be excluded from the micro-data file before being released to prevent confidential information from being linked to specific respondents. Generally speaking, variable of this type can lead to a direct disclosure $[45,60,142]$.

- Quasi-identifying Variables

Quasi-identifiers constitute a set of variables in the micro-data file that, in combination, can be linked with any external information. These permit the attacker to re-identify the respondent to whom the records in the micro-data file refer to [142]. Variables of this kind could lead to indirect disclosures [45]. The problem here is that it is not possible to eliminate quasi-identifiers because any variable in the micro-data file can potentially be a quasi-identifier. Indeed, there is no "God-given" procedure which can assist in deciding whether a variable belongs in this class or not.

- Sensitive Variable

A sensitive variable is an attribute that is found in the released data, but which is, typically, unknown to an intruder before the data is released. This attribute 
contains non-confidential information that could enable the accurate estimation of confidential information. Examples of such sensitive variables are Religion, Health_Condition, etc $[20,60,89,142]$.

- Safe Variable

Attributes of this class contain non-confidential information pertaining to the respondent. Additionally, such a variable does not add any "critical" information to the confidential data. Examples of such a variable could be Town, Country, Nationality, etc. In protecting a data set, we should also consider the fact that an attribute of this kind could, without our knowledge, also be a quasi-identifier $[20,60,89,142]$.

\subsection{Micro-data Performance Measures}

As mentioned in Section 2.1, the purpose of $S D C$ can be given quite informally by stating that, given the original micro-data set, $V$, the goal is to release a protected micro-data set, $V^{\prime}$, in such a way that $[102,60]$ :

- Disclosure Risk, (i.e., the risk that a user or an intruder can use $V^{\prime}$ to determine confidential sensitive attributes on a specific individual among those in $V$ ), is low.

- User Analysis on $V^{\prime}$ and on $V$ yield the same or at least similar results.

\subsubsection{Micro-data Disclosure Risk}

Micro-data $S D C$ attempts to protect the privacy of individual data in the released data set $V^{\prime}$. The issue of disclosure is far from being trivial. It would occur if the released data enabled the intruder to identify or determine the value of the sensitive confidential information with a fair degree of certainty $[89,142]$. We refer to this 
kind of disclosure as "Predictive Disclosure", because it concerns the prediction of the unknown (or unspecified) value of a variable. If the predictive disclosure can be made with certainty, it is referred to as being a "deterministic" predictive disclosure. This is contrasted with a "probabilistic" predictive disclosure, which involves knowing the unknown value within a probabilistic framework. The deterministic type of predictive disclosure can further be classified as being either "exact" or "approximate". In the exact case, the intruder predicts the exact value of the confidential information, while in the approximate case the intruder is able to deduce an interval for the confidential value $[123,142]$. Other common types of disclosure are listed below $[89,117]$ :

- True Disclosure. This occurs when the exact or approximate value of the sensitive data represents the real status of the attribute at the time of dissemination.

- Apparent Disclosure. This occurs when the exact or approximate value of the sensitive data represents a reasonable, but not the exact, value of the attribute at the time of dissemination.

- Positive Disclosure. This occurs when the information learned pertains directly to the identified unit.

- Negative Disclosure. This occurs when the information learned docs not pertain directly to the identified unit but to its compliments.

Since $D R$ can be defined as the risk caused by using the protected data set $V^{\prime}$ to deduce the confidential information on an individual found in the original data set $V$, it can be viewed from two different perspectives [23]:

- Attribute Disclosure

This occurs when the attribute of an individual can be determined more accurately when the user has access to a related statistic, as opposed to the case when the user does not have this access. In other words, attribute disclosure occurs when the respondent can be associated with either the exact attribute value 
in the disseminated data or an estimated (approximated) attribute value based on the disseminated data [23]. In this case, a rank-based interval technique can be used to measure the attribute disclosure. Each attribute in the protected data set, $V^{\prime}$, is ranked independently. A rank interval is defined around the value that the attribute takes in each record, $r$. The ranks of values within the interval for an attribute for records around $r$ should differ by less than a certain percentage of the total number of records, and the rank in the center of the interval should correspond to the value of the attribute in record $r$. If this is true, the proportion of original values that fall into the interval centered around their corresponding protected values is a measure of $D R$. For more information about this metric, the reader is referred to $[23,44,48,53]$. Further details about this technique will be included in Chapters 4 and 5 .

\section{- Identity Disclosure}

This occurs when a record in the protected data set can be linked with a respondent's identity. In other words, the identity disclosure permits a respondent's identity to be associated with the disseminated data records. This association is assumed to be exact $[23,44,48]$. Identity disclosure can be further classified as into two categories.

\section{- Uniqueness}

The risk of identity disclosure can be measured as the probability that rare combinations of attribute values in the set $V^{\prime}$ are also rare in the set $V$. Such a quantifying approach is used in the context of non-perturbative $S D C$ methods $[23,44,48]$. Here, we further distinguish two kind of measures: File-level risk measures and record-level risk measures. In the case of file-level risk measures, $D R$ is defined as the probability that a sample that is unique is also unique to the entire population. Generally, the size of the sample is much smaller than the size of the population. If this was not the case, an intruder would be able to locate the unique value in the relcased sample, which, in turn, would lcad to the identification of the individual respondent in the original population [53]. A record-level risk 
measure is defined as the probability that a particular sample record is re-identified. The author of [63] integrates two methods for measuring $D R$ at the record-level with file level risk measures. More details of these $D R$ measures can be found in $[23,44,48,72,77]$.

\section{- Re-identification}

The concept of re-identification quantifies the estimate to the number of re-identifications that can be obtained by an experienced intruder. Reidentification methods are schemes used to measure the $D R$, and they provide more unified approaches to this problem than the uniqueness methods. This is because a re-identification method can be applied to both perturbative and non-perturbative $S D C$. In this case, a record linkage technique is used to measure the identity disclosure in $S D C$. It consists of linking each record, $a$, in the set $V^{\prime}$, to a record, $b$, in the set $V$. The pair $(a, b)$ is a match if the record $b$ turns out to be the record $a$. It is important to mention that measuring the risk of identity disclosure also assumes that the intruder has an external data set that shares some attributes with the protected data set and that also contains some identifier attributes. Thus, the intruder has to link the protected data set with the external data set using the shared attributes. The number of matches will then represent the number of protected records whose corresponding respondent values can be re-identified by the intruder. The main types of record linkage, which are typically used to measure the identity disclosure in $S D C$, are discussed below [23, 44, 48].

\section{* Distance-based Records Linkage}

Distance-based record linkage measures the scenario when each record $a$ in the set $V^{\prime}$ is linked to its nearest record $b$ in the set $V$. In such a case we require a definition of a distance function for expressing nearness between records, and there also is a need to standardize attributes (so as to avoid scaling problems) and to assign each attribute a. weight on the record-level distance. The linkage computation then proceeds by evaluating the distances between records in the sets $V$ 
and $V^{\prime}$. To do this, the distance to every record in the set $V$ is first computed. Thereafter, the "nearest" and "second nearest" records in $V$ are considered. A record in the set $V^{\prime}$ is labeled as being "linked" when the nearest record in the set $V$ has the same record number (or index), while a record in $V^{\prime}$ is labeled "linked to second nearest" when the second nearest record in the set $V$ has the same record number (or index). In all other cases, the record in $V^{\prime}$ is labeled as being "not linked". The main advantages of using a distance measure for record linkages are simplicity for the implementer and intuitiveness for the user. The main difficulty appears in determining an appropriate distance for categorical attributes, and for masking methods such as those which use local recording when the masked protected file contains new labels with respect to $V$. Finally, this technique is used to measure the $D R$ of the micro-aggregation in the context of the Euclidean distance $[23,44,48,53]$. The authors of [132] explore the use of the Mahalanobis distance, which leads to a better estimation of the true dissimilarity, and thus to a higher level of protection against attackers. This techniques will also be discussed in more details in Chapters 4 and 5.

* Probabilistic Record Linkage

Probabilistic record linkage aims at linking pairs of records $(a, b)$ in the sets $V^{\prime}$ and $V$, respectively. The user needs to provide as input two probability values: The maximum acceptable probability of linking a pair that is an unmatched pair, $(L T)$, and the maximum acceptable probability of not linking a pair that is a match, $(N L T)$. For each pair, the user computes the corresponding indices. Then, $L T$ and $N L T$ in the index range are used to label the pair as being either linked, clerical, or non-linked as follows: If the index is above $L T$, the pair is linked; if it is below $N L T$, the pair is non-linked; if it is between $L T$ and $N L T$, the pair is clerical, which is the case when the pair cannot be automatically classified but requires human inspection. 
The measure for $D R$ is then defined as the number of matches over the number of records in the set $V^{\prime}$. Although the probabilistic record linkage scheme is more sophisticated than the distance-based one, it does not, on the other hand, require a re-scaling or a weighting of the attributes $[23,44,48,53,146]$, and is, thus, often a preferable option.

* Other Record Linkage Methods

Recently, the use of other record linkage methods has also been considered for assessing $D R$. While, previously, the record linkage methods assumed that the two files contained shared variables, other methods that relaxed this condition have been recently developed. Some methods use the correlations between the variables, or auxiliary population files, to improve the linkage accuracy. Alternative methods use Markov Random Files, or graph partitioning algorithms so as to increase the likelihood of a set of linkages between corresponding records in a group of files. It should be mentioned that these most recent methods are often used for extracting and linking information from a group of webpages [144].

\subsubsection{Micro-data Information Loss}

The degradation of the data, of course, reduces the ability of data users to conduct the analysis they need for legitimate purposes. These effects fall into two categories $[21,72]$ :

- Reduction of analytical completeness

Some control methods, (typically those involving the recoding of taxonomic schemes into coarser categorizations), that could have been conducted with non-recoded data are not achievable.

- Loss of analytical validity

The loss of analytical validity is harder to define, but in some ways more critical 
because of its insidious nature. Technically, loss of validity can be said to occur when a disclosure control method has changed a data set to the point where a user arrives at a conclusion different from what a similar analysis on the original data set would have led to.

The loss in the content of information depends on the usage of data. Data is usually used in various areas, and it is very difficult to identify all its uses at the time of dissemination. Additionally, assuming all data uses can be identified, issuing several versions of the same original micro-data may result in unexpected disclosure. Thus, it is more reasonable to measure the amount of the $I L$ in a generic way, which estimates how much the data is harmed by using any $S D C$ method [21, 55, 93]. Generally speaking, the $I L$ is very small, if the analytical structure of the micro-data file, after the masking is very similar (nearly identical) to its structure before the masking. In fact, preserving the structure of the micro-data file is very important to ensure that the new masked file will be analytically valid and interesting $[44,48,93]$.

It is important to give a clear definition for both the terms "analytically valid" and "analytically interesting" [21, 55, 93].

- Analytically Valid. The micro-data file is analytically valid if, with respect to the original file, it approximately preserves the following:

1. The means and covariances on a small set of sub-domains.

2. The marginal values for a few tabulations of the data.

3. At least one distributional characteristic.

- Analytically Interesting. The micro-data file is analytically valid if at least six attributes on the important sub-domains, which can be validly analyzed, are provided.

It is difficult to come up with a precise description of analytical validity and analytical interest without determining where the data will be used. But, several 
complementary ways are highlighted to ensure the preservation of the structure of the original data $[44,48,55,93]$ :

1. Compare the original micro-data with the masked micro-data. The more similar the $S D C$ method is to the "identity" function, the less impact there is on the information content.

2. Compare some statistics computed on the original and masked micro-data. Small differences between the statistics yield to little loss in the information content.

3. Analyze the behavior of the particular $S D C$ method used to measure its influence on the structure of the original micro-data.

An automatic data modification procedure can be used to measure the amount of the $I L$. Theoretically, if the original micro-data file is considered as a message to be transmitted, and the masked micro-data file as the transmitted message, and the modification that separates them as a noisy channel, then the idea is to use information theoretic concepts to quantify the $I L$ quickly follows. Although this idea is possible to some extent, it is not practical because it is time-consuming [142].

Generally speaking, a data protector who protects the micro-data file usually knows how to protect the data and how to optimize the loss in the information content. Thus, a formal measure for the $I L$ is used in order to compare the different possibilities of getting a safe micro-data file [142]. It is important to highlight the formal measures used to estimate the $I L$, which can be classified into two categories based on the type of the used data $[21,44,48,55,93]$ :

\section{Information Loss Measure for Continuous Data}

There is no single quantitative measure that reflects the difference between the original and the masked continuous micro-data file. Therefore, the following tools are used to characterize the information contained in the micro-data file 
$[21,44,48,55,93]$. It is most likely that these various tools are used to measure the loss in the data utility, (i.e., the loss of the accuracy sought by database users), through the discrepancies between the matrices $X, V, R, R F, C$, and $F$ obtained from the original data and the corresponding $X^{\prime}, V^{\prime}, R^{\prime}, R F^{\prime}, C^{\prime}$, and $F^{\prime}$ obtained from the protected micro-data file, where:

$$
\begin{aligned}
X \text { and } X^{\prime}: & \text { are the matrix representing the original and protected } \\
& \text { micro-file, respectively, which consists of } n \text { records } \\
& \text { and } p \text { attributes. } \\
V \text { and } V^{\prime}: & \text { are the covariance matrices of } X \text { and } X^{\prime} \text {, respectively. } \\
R \text { and } R^{\prime}: & \text { are the correlation matrices of } X \text { and } X^{\prime} \text {, respectively. } \\
R F \text { and } R F^{\prime}: & \text { are the correlation matrices between the } p \text { factors } \\
& P C_{1}, \ldots, P C_{p} \text { obtained through principle components } \\
& \text { analysis from } X \text { and } X^{\prime} \text {, respectively. } \\
: & \text { are the commonalities between each of the } p \text { variables } \\
& \text { and the first principle component } P C \text { for } X \\
& \text { and } X^{\prime}, \text { respectively. } \\
: & \text { are the factor score coefficient matrices, which contain } \\
& \text { the factors that should multiply each variable in } X \text { and } \\
& X^{\prime}, \text { respectively, to obtain its projection on each. } \\
& \text { principle component. }
\end{aligned}
$$

Matrix discrepancy can be measured in at least three ways $[21,44,48,55,93]$.

(a) Mean square error

Sum of squared component differences between pairs of matrices, divided by the number of cells in either matrix.

(b) Mean absolute error

Sum of absolute component differences between pairs of matrices, divided by the number of cells in either matrix.

(c) Mean variation

Sum of absolute percent variation of components in the matrix computed 
on protected micro-data with respect to components in the matrix of the original micro-data, divided by the number of cells in either matrix. This approach is not affected by scale changes of the attributes. In other words, in this case, there is no need to standardize the attributes.

Measuring the $I L$ using any (or all) of the various tools, which have been discussed above, has a major disadvantage: The measure is only defined as a function of the protected masked micro-data, which does not depend on the original micro-data. The authors of [55] measure the information loss for a variable, $V$, as a function of three elements: The condition probability, the original micro-data $i$, and the masked category $j$. Thus, the per-record $I L$ when $V=i$ is masked as $V^{\prime}=j$ can be defined as:

$$
P\left(P_{V, V^{\prime}}, i, j\right)=-\log P\left(V^{\prime}=j \mid V=i\right) .
$$

Measuring the $I L$ for the whole micro-data file is then defined as:

$$
P\left(P_{V, V^{\prime}}, O, M\right)=\sum_{r \varepsilon M} P\left(P_{V, V^{\prime}}, i_{r}, j_{r}\right)
$$

where $i_{r}$ is the value taken by the variable $V$ in record $r$ of the original microdata file, $O$, and $j_{r}$ is the value taken by the variable $V^{\prime}$ in record $r$ of the masked micro-data file, $M$ [93].

Finally, it is worth mentioning that the quantity $I L$ is to be traded-off with the $D R$. Since $D R$ is bounded, there is no risk higher than 100 percent. On the other hand, the upper bound does not practically exist, but it should be enforced for $I L$ measures [21]. The authors of [93] have shown a way to obtain probabilistic information loss measures for assessing the impact of $S D C$ methods on continuous micro-data sets. Being probabilistic, a value between 0 and 1 makes the protection easier, by finding an optimal balance between $I L$ and $D R$.

For more information about the various tools that are used to estimate the amount of the $I L$, and on measuring the matrix discrepancy, the reader is 
referred to $[21,44,48,55,93]$. The question of estimating the general $I L$ will be explained in more detail in Chapters 4 and 5 .

\section{Information Loss Measure for Categorical Data.}

Applying the above measures on categorical data is not possible. For categorical data, three kinds of $I L$ measures have been proposed in the literature [21, 44, $48,55]$ :

\section{(a) Direct Comparison of Categorical values}

The comparison of the original and the protected matrices requires a clear definition of the distance for categorical attributes. The distance is measured by considering the distance between pairs of categorical values, when comparing a record in the original micro-data file and its corresponding record in the masked version $|21,55|$.

Measuring the distance for nominal attributes only uses the comparison of equality. But for the ordinal attributes, the distance is measured by the total number of categories between them, measured over the cardinality of the range of the ordinal attribute. More information on this issue can be found in $[21,55]$.

(b) Comparison of Contingency Tables

An alternative strategy to the direct comparison of the values in the case of categorical variables is to compare their contingency tables. Contingency tables are compared for a file before and after applying the masking method. The number of differences between both contingency tables quantifies the amount of the $I L$. Usually, the number of cells in the contingency table depends on the number of categories in the variable; thus, a normalized version exists, which is exactly the number of differences between the two contingency tables divided by the number of cells in all the tables considered. For more information about such a metric, the reader is referred to $[21,44,55]$.

(c) Entropy-Based Measures

Entropy is essentially an information theoretic measure, but it can be used 
in $S D C$ if the masking process is modeled as the noise that would be added to the original micro-data in the event of its being transmitted over a noise channel $[21,83]$ : The use of entropy-based methods is limited to PRAM, because of the issues involving generalized noise addition, local suppression, and global recoding methods [48].

Let $V$ be a variable in the original micro-data file and $V^{\prime}$ be the corresponding variable in the masked file. The condition uncertainty of $V$ given $V^{\prime}$ can be deduced from the Markov matrix in PRAM as follows [21, 48]:

$$
H\left(V \mid V^{\prime}=j\right)=-\sum_{i=1}^{n} P\left(v=i \mid V^{\prime}=j\right) \log P\left(V=i \mid V^{\prime}=j\right) .
$$

Thus, the loss in the information content can be calculated by accumulating the above expression for all individuals in the masked micro-data file, $M$ as $[21,48]$ :

$$
I L\left(P_{V, V^{\prime}}, M\right)=\sum_{r \varepsilon M} H\left(V \mid V^{\prime}=j_{r}\right)
$$

\subsubsection{Trade-off between DR and IL}

Government agencies collect various types of data, but due to access restrictions, micro-data is usually accessed using a secure computer system. Of course, this access restriction affects public policy decisions made by any agency that has access to the non-confidential statistical summaries. This requirement to access the original microdata should not only satisfy the confidentiality requirements, but also the sufficient utility [82].

There is a very broad choice of methods for continuous micro-data protection (see Section 2.1). Most of these methods are parametric (i.e., in the micro-aggregation technique one parameter is the minimal number of records per group). Therefore, the user has to go through two choices rather than a single choice: A primary choice to select the masked method and a secondary choice to set an appropriate value for the parameters of the selected method [93]. 
The optimal way to choose the appropriate method and parameter should yield to an optimal trade-off between $I L$ and $D R$. This brings us to the need to combine the measures of $I L$ with those related to $D R$. Generally speaking, there are two approaches to do this [93]:

\section{Explicit}

This method adapts a score for the method performance rating. Typically, this method combines the average values of the $I L$ and $D R$. Using a score defines a new optimization problem by selecting a masking method and setting the appropriate values for its parameters [93].

\section{Implicit}

Methods of this family assume that there is no specific score that can do justice to all methods, for all data uses, and for all disclosure scenarios. Thus, it is the responsibility of the data protector to separately compute several $I L$ and $D R$ measures, and to choose the most appropriate method based on a combination of the most relevant measures for specific data use/disclosure cases [93].

It is important to present a general principle that shows how to balance the trade-off between $I L$ and $D R$. There are various strategies to obtain and reach the equilibrium point between them [27]. If $R$ represents the disclosure risk and $U$ the utility, one strategy involves computing $R-U$ for each method, $S$, and parameter, $P$. The score is computed as follows: $\operatorname{Score}(O, M)=\frac{I L(O, M)+D R(O, M)}{2}$, where $M$ is the protected file obtained after applying method $S$ with parameterization $P$ to the original file. Otherwise, the $U-R$ map, which is a graphical representation tool, is used. According to [27] "in its most basic form, $R-U$ map is the set of paired values $(R, U)$ of $D R$ and data utility that correspond to many strategies for data release". Such $(U, R)$ pairs are plotted on a two dimensional graph, so that the user can easily understand the influence of a masked method and/or the parameter choice [27].

A strategy that compromises between preserving the confidentiality requirements and maximizing the data utility was introduced by Kim et al. in [82]. The basic idea 
here is to mask the multi-variate normal quantitative data using an additive noise approach and then, when it is necessary, use a re-identification/swapping approach to assure confidentiality. The most important advantage of this strategy is that of obtaining exact sub-population estimates, because this involves applying a controlled distortion that renders the estimates from the masked file nearly identical to those from the original file. This method has been extended to mask non-normal multivariate data by involving transformations that preserve the essential characteristics of the data such as its mean, variance, and correlations [129].

The authors of [124] state that they can improve the performance of any specific $S D C$ method by involving a post-masking optimization technique. The latter strategy preserves the moments of the first and second order by minimizing the $I L$ between the original and the masked micro-data file. Simultaneously, it does not increase the $D R$ because it always prevents the optimized masked file from being very close to the original file.

In conclusion, consideration must also be given to the conceptual framework, since one must decide whether to publish the information after using one of the $S D C$ techniques or not. This decision is a crucial one, because we have to estimate the loss associated with the possibility of disclosure and, simultaneously, the loss attained by not publishing the data. A Bayesian model can be used to make this critical decision. The final decision of whether to publish the data or not is determined by evaluating whether the risk of publication exceeds the loss of non-publication [150].

\subsection{Micro-data Protection}

Micro-data protection methods usually generate a new released version of the statistical data by either of the following schemes [20]:

- Generating synthetic data

Using synthetic (or simulated) data was proposed decades ago as a method to 
guard against $D R$ [26]. Instead of releasing the real data, the scheme suggests generating a set of synthetic micro-data from a model fitted to the real data. The idea is to randomly generate data with the constraint that certain statistical or internal relationships of the original micro-data are preserved [142]. It would appear as if the synthetic data would have the advantage of circumventing the re-identification problem, because the published records are "invented" and are not derived from the real data. This is, however, not the case, because the synthetic data usually over-fits the original data, which may, in turn, be disclosed in the same way as the original data $[20,29,144]$. On the other hand, an obvious problem associated with using synthetic data is its data utility [47]. The only preserved statistical properties are those that are explicitly selected by the data protector $[20,47,142]$. More information about these methods can be found in [26].

- Masking original data

Masking methods modify the original data in a special way. These modifications seek to preserve the confidential information, while providing valid and interesting analytical statistical information. SDC methods for micro-data are usually known as "masking" methods. Generally speaking, masking methods can be classified based on two different points of view $[20,55,142]$ :

\section{- Operational principle}

Masking methods that work on the basis of an operational principle can, in turn, be further classified into two categories depending on their effect on the original data $[20,142,53,55]$ :

* Perturbative

The confidentiality of the statistical data is preserved by distorting the micro-data. This distortion may add new and unique combinations to the released version of the data, or it could delete some unique combinations of scores. All perturbative methods should provide the data set with analytical results similar to the results that would be obtained from the original data set $[20,25,55]$. 


\section{* Non-perturbative}

These methods produce partial reduction of the details in the original data, instead of harming and altering the original data $[20,24,55]$. This can be achieved by making sure that every unsafe combination must not have a frequency below a predefined threshold value [142].

\section{- The data type on which they are used}

This is an alternate classification of the masking methods based on the type of data that are used. This classification divides the masking methods into two parts: (i) Continuous, in which case the $S D C$ methods are applied on continuous variables, and (ii) Categorical, which is the case when the $S D C$ methods are applied on categorical variables $[20,55]$.

\section{- Generating hybrid micro-data}

The third possibility to build a hybrid data set as a mixture of the masked original values and synthetic data set is to combine a masking method with Cholesky's decompositions [47]. A hybrid micro-data file offers numerous advantages when compared to the traditional micro-data files: (i) It overcomes the lack of analytical validity by preserving both the uni-variate and multivariate statistical properties of the original micro-data file, such as their means, covariances, and correlations. Besides this, it also preserves a fair amount of record-level similarity between the released and original files, which, in turn, allows sub-domain analysis [47]. (ii) The method is very fast, because its running time is linear in the number of records. (iii) It overcomes the identification problem by publishing the simulated data and not the original real data.

In this research, we will focus on masking methods, and an overview of the state-ofthe-art of these methods will be covered in detail in Sections 2.5.1 and 2.5.2. 


\subsubsection{Perturbative Masking Methods}

Perturbative methods release the entire original data. But, instead of releasing the exact value of the attributes, a perturbed value is released for each attribute. It is important to mention that not all the perturbative methods are designed for continuous data. Table 2.1 indicates whether the perturbative methods are suitable for continuous and/or categorical data $[25,55,76]$.

Table 2.1: Perturbative methods for various data types.

\begin{tabular}{|c|c|c|}
\hline Perturbative Methods & \multicolumn{2}{|c|}{ Data types } \\
\cline { 2 - 3 } & Continuous & Categorical \\
\hline \hline Additive Noise & $\mathrm{X}$ & \\
\hline Micro-Aggregation & $\mathrm{X}$ & $\mathrm{X}$ \\
\hline PRAM & & $\mathrm{X}$ \\
\hline Data Swapping & $\mathrm{X}$ & $\mathrm{X}$ \\
\hline Rounding & $\mathrm{X}$ & \\
\hline Resampling & $\mathrm{X}$ & \\
\hline Lossy Compression & $\mathrm{X}$ & \\
\hline \hline
\end{tabular}

The techniques which are known as additive noise, micro-aggregation, data swapping, and post-randomization are special cases of matrix masking. The released set $V^{\prime}$ can be computed from the set $V$ as follows:

$$
V^{\prime}=A V B+C
$$

where $A$ is a record-transforming mask, $B$ is a variable-transforming mask, and $C$ is a displacing mask (noise) $[25,55,102]$. We detail these methods below.

- Additive Noise

Masking with additive noise is one of the data perturbative techniques that statistically seeks to protect confidential micro-data by adding random noise to confidential numerical attributes, where the noise possesses the same correlation structure as the original data $[13,55,102]$. Note that this technique 
does not belong to one of the encryption techniques, where the data is initially modified by a secure encryption, then transmitted, and finally the received data is "decrypted" by the user.

The main additive noise algorithms reported in the literature are: Masking by adding uncorrelated noise, masking by adding correlated noise, masking by adding noise involving linear transformations, and masking by adding noise involving non-linear transformations. For more details on the specific algorithms for each of these topics, the reader is referred to $[13,19,76]$.

Most masking methods combine transformations by adding noise [50, 70]. White noise is most frequently used, even though it may be subject to the bias problem. Despite this bias problem, white noise is still built into the $\mu$-ARGUS software package $[48,55,77,102]$. Studies demonstrate that additive noise perturbative techniques exhibit bias when the results of a database query on perturbed data produces a significantly different "result" than the same query executed on the original data [72]. Five types of biases have been identified as [143]:

- Type $A$ bias occurs when the perturbation of a given attribute causes summary measures (i.e., mean value) of that individual attribute to change due to a change in variance.

- Type $B$ bias occurs when the perturbation changes the relationships (e.g., correlations) between the confidential attributes.

- Type $C$ bias occurs when the perturbation changes the relationship (again, e.g., correlations) between confidential and nonconfidential attributes.

- Type $D$ bias deals with the underlying distribution of the data in a database, specifically on whether or not the data has a multi-variate normal distribution.

- Type $D M$ bias could severely impact the ability of an organization to gain significant benefits of knowledge management/discovery from the disclosed data.

Finally, the nature of additive noise makes it unsuitable for categorical data. 
On the other hand, it is well suited for continuous data for the following reasons $[25,55,102]$ :

- There are no assumptions on the range of possible values for the attributes.

- The random noise, which is added to the original data, is typically continuous and has a mean of zero.

- Approximate matching is possible with external files, because it depends on the amount of noise that is added.

\section{- Micro-Aggregation}

Micro-aggregation is a perturbative technique applicable to quantitative variables [142]. The rationale behind this technique is that confidentiality rules allow publication of the original data, if records correspond to groups of $k$ or more individuals, where no individual dominates the group and $k$ is a threshold value [55]. The basic principle of micro-aggregation is as follows: Records are clustered into small aggregates or groups of size at least $k$. Rather than publishing the original variable for a given individual, the average of the values over the group to which the individual belongs is published [34, 45, 55, 102]. Micro-aggregation can be viewed as a technique like rounding or noise addition, although it has the property of preserving the grand total of the data involved [142]. In spite of keeping the total for a variable intact, it has a tendency to reduce the variances of the variables to which it is applied [142].

Generally speaking, generalizing uni-variate micro-aggregation to yield a multivariate micro-aggregation scheme can be done using several variants such as fixed vs. variable group size $[45,92,122]$, exact optimal vs. heuristic microaggregation [45, 92, 77, 103], and categorical micro-aggregation [131, 56, 60].

Finally, individual micro-aggregation, micro-aggregation using $z$-scores projection and principle component projection, and micro-aggregation on unprojected multi-variate data considering two variables at a time, three at a time, four at a time, or all variables simultaneously are built into the $\mu$-ARGUS Software $[48,76,77,102]$. 
In this research, we will focus on techniques within this family. They will, thus, be covered in greater detail in a subsequent section. Our primary results in this research endeavor deal with novel micro-aggregation strategies.

\section{- PRAM}

The Post-RAndomization Method $(P R A M)$ is a probabilistic, perturbative method that has been inspired by the so-called randomized response technique [142]. It is used to protect the categorical attributes in the micro-data file [25]. PRAM produces a new masked file by changing the scores of some variables for certain records in the original file, according to a prescribed probability mechanism (typically, a Markov matrix) [83]. The random procedure in PRAM methods is based on matching a record in the masked file with a known individual in the original file (which occurs with a very low probability) [73]. The Markov approach makes PRAM very general, because it consists of noise addition, data suppression, and data recording [25].

Generally speaking, although the $I L$ and $D R$ issues associated with a PRAM technique largely depend on the choice of the Markov matrix, (which is still an open research topic [145]), it has been implemented in the $\mu$-ARGUS Software package $[48,76,77,102]$. Finally, it is worth mentioning that the application of this method on continuous data is still open, since to-date, to the best of our knowledge, PRAM has only been applied on categorical data [25].

- Data Swapping and Rank Swapping

Data swapping is considered a special case of PRAM [142]. The basic idea behind this method is to apply a sequence of elementary swaps of confidential attributes between individual records in the micro-data files. Records are exchanged in such a way that lower-order frequency counts (or marginals) are maintained $[25,142]$. An elementary swap consists of two actions:

1. A random selection of two records, say $i$ and $j$, from the micro-data set.

2. An interchange of the values of the variable being swapped for records $i$ and $j$. 
The basic rationale for data swapping has also left a marked impact on subsequent methods, as reported in [25].

Another important variant of the data swapping strategy is Rank Swapping [44]. The latter method was originally proposed for ordinal categorical variables. But since then, it has also been used for other numerical variables [96]. To achieve this, first of all, values of an attribute are ranked in an ascending order, and then each ranked value is swapped with another ranked value that is randomly chosen within a restricted range (i.e., the rank of two swapped values cannot differ by more than $P \%$ of the total number of records, where $P$ is an input parameter). This algorithm is independently used for each original attribute in the original data set $[44,48,55]$. The authors [53] argue that a rank swapping method can be identified as being a method that yields a good trade-off between $D R$ and $I L$. Consequently, this scheme has also been implemented in the $\mu$-ARGUS Software package $[48,76,77,102]$.

\section{- Rounding}

The rounding method is used only for continuous data. It replaces the original value of a variable by the rounded value ${ }^{4}[55]$. For a given variable, rounded values are chosen from a set of rounding points defining a rounding set. This value can either be chosen deterministically or randomly. An example of a deterministic rounding procedure is to round a value to the nearest integer multiple of the rounding base. A stochastic random rounding procedure could round each value, with certain probability, to an integer multiple of the rounding base [142]. In the case of multi-variate micro-data, rounding is usually applied to one variable at a time (uni-variate rounding), although examples of multivariate rounding have been reported [55].

- Resampling

The resampling method was originally proposed to protect tabular data, but resampling can also be used to protect numerical micro-data $[43,27]$. The

\footnotetext{
${ }^{4}$ The rounded values are integer multiples based on a well-chosen rounding base [142].
} 
basic idea is taking $t$ independent samples $S_{1}, S_{2}, \ldots, S_{t}$ of size $n$ of the values of a variable $X$. Then, the values are independently ranked using the same ranking criterion. Finally, the masked variable $X^{\prime}$ as $x_{1}^{\prime}, x_{2}^{\prime}, \ldots, x_{n}^{\prime}$, (where $n$ is the number of records) is computed for each value, where $x_{i}^{\prime}$ is obtained by evaluating the average value of the $i^{\text {th }}$ ranked value in each sample $S_{1}, S_{2}, \ldots, S_{t}$ $[25,44,55,102]$. Resampling has been implemented in the $\mu$-ARGUS Software package, and it has been tested for $t=1$ and $t=3[48,77,102]$.

\section{- Lossy Compression}

Lossy compression is a new method that can be applied to continuous data[102]. The basic idea is to consider the micro-data file as an image (where records are rows, variables are columns, and the values themselves are pixels ${ }^{5}$ ). A lossy compression method is used on the micro-data file as follows: First of all, the original micro-data values have to be scaled to be integers into the interval of the gray-scale values, in order to produce a gray-scale image. Secondly, a compression algorithm (i.e., JPEG) is applied to yield a certain percentage, $P \%$, quality on the image. Finally, a masked file is generated by unscaling (using the inverse of the scale transformation used in the first step) the image $[25,44,48]$. Here, it is important to mention that in spite of requiring an appropriate mapping between the attribute ranges and the color scales, (which depends on the compression algorithm used [55]), lossy compression methods have been implemented in the $\mu$-ARGUS Software package. More information of such methods can be found in $[48,77,102]$.

\subsubsection{Non-Perturbative Masking Methods}

As mentioned in Section 2.5, non-perturbative methods do not destroy the original data, but reduce the detail in the original file. Some similar methods have been applied for categorical and continuous data, but others have only been suitable for categorical data. Table 2.2 lists the non-perturbative methods, and also indicates the

\footnotetext{
${ }^{5}$ Typically, for a black and white image, the value of the pixel is its gray-scale level [55].
} 
data for which each method is suitable [24]

Table 2.2: Non-Perturbative methods for various data types.

\begin{tabular}{|c|c|c|}
\hline \multirow{2}{*}{ Non-Perturbative Methods } & \multicolumn{2}{|c|}{ Data types } \\
\cline { 2 - 3 } & Continuous & Categorical \\
\hline \hline Sampling & & $\mathrm{X}$ \\
\hline Global Recoding & $\mathrm{X}$ & $\mathrm{X}$ \\
\hline Top and Bottom Coding & $\mathrm{X}$ & $\mathrm{X}$ \\
\hline Local Suppression & & $\mathrm{X}$ \\
\hline \hline
\end{tabular}

- Sampling

Masking with a sampling method requires publishing a sample (or a subset) of the original records in the micro-data file, instead of publishing the entire records in the original file [55]. A sampling method is suitable for categorical micro-data, but its adequacy for continuous data is less clear. The main reason behind this is that the sampling method leaves the continuous attribute unperturbed for all records in the sample $[24,55]$. This, of-course, can be easily identified, especially if the intruder matches the value of the attribute in the masked file with another value in an external administrative public file [144]. More information about the technical specifications of a real-world application of sampling can be found in [24].

- Global recoding

Global recoding schemes, which are intensively used by statistical offices, are more appropriate for categorical micro-data, because they help disguise records with unusual combinations of categorical attributes [24, 55]. In such a scheme, for a categorical attribute, several categories are combined to form a new category, and thus the resultant category should satisfy $\left|D\left(V_{i}^{\prime}\right)\right|<\left|D\left(V_{i}\right)\right|$, where |.| is the cardinality operator $[24,55,102]$. In other words, two or more categories of a variable in a micro-data file are combined into a single one, and the same coding is used for all the units. Of-course, reducing the number of 
categories of a variable implies a reduction of the information content of the variable concerned [142].

Global recoding has been used for continuous variables by replacing the continuous variable, $V_{i}$, with another discretized version of $V_{i}$. In other words, a potentially infinite range $D\left(V_{i}\right)$ is mapped into a finite range $D\left(V_{i}^{\prime}\right)[24,55]$. The reader has to remember that by discretization, one inherently obtains an unaffordable loss of information. Also, arithmetic operations that were straightforward on the original $V_{i}$ may be easily applied on the discretized version [102]. Implementing the process of global recording in computer software may either take place in an automatic way, where the choice of categories that are to be combined is determined according to an objective criterion, or in an interactive way, where the choice of categories to be combined is left to the user of the software [142]. This technique has also been implemented in the $\mu$-ARGUS package $[48,76,77,102]$.

- Top and bottom coding

Top-down coding is a special case of the global recoding technique. It is used on variables that can be ranked, such as continuous and ordinal categorical data $[24,44,48,102]$. While a top category covers the values of the variable above a certain upper threshold, $\theta_{U}$, a bottom category covers the values below another specified lower threshold, $\theta_{L}$. The critical practical problem encountered in this technique is that of determining the appropriate values for $\theta_{U}$ and $\theta_{L}$ [142]. This method has also been implemented in the $\mu$-ARGUS software package [77]

- Local suppression

Local suppression is a non-perturbative technique that involves deleting a value in a record and replacing it by a missing indicator $[24,55]$. This procedure is applied to a single record at a time, and it does not require the deletion of all the values of the same variable. Thus, local suppression is not harmless when it comes to imputing values for missing variables [142].

In practice, local suppression is combined with global recoding, (which is the 
primary $S D C$ technique for a micro-data file), so as to remove a few remaining instances of unsafe records [142].

Generally speaking, this techniques is more applicable for categorical data than for continuous data. This is because, sometimes, the continuous attribute is a component of a set of key attributes, which renders each combination of key values unique. Since the suppression of the values of a continuous attribute is meaningless, it is generally assumed that local suppression cannot be used for continuous variables [24].

\subsection{Micro-Aggregation Techniques (MATs)}

\subsubsection{Introduction}

At present, a large number of agencies have collected useful data suitable for research purposes. A decision to prohibit these useful pieces of information from being disseminated has sometimes been made in an attempt to preserve the confidential nature of the data. Therefore, as explained earlier, there is a tacit conflict between the principle of privacy and the need to discover new characteristics found in the data. As, in practice, neither principle can be totaly preserved, often, a trade-off between them is acceptable, and in order to access the confidential micro-data at lower costs, we have seen that it is expedient to apply an $S D C$ technique.

As explained earlier, various methods have been proposed and applied to ensure a good measure of confidentiality and data utility. Each method either provides a modified version of the entire data set, or the original exact data set where sensitive values of the variables are either erased or re-coded [115].

One of the most recent techniques proposed is the strategy called "Micro-aggregation". The latter comprises of a family of statistical disclosure limitation techniques used to protect micro-data files, each of which contains records on individual data subjects. 
These belong to the family of substitution/perturbation approaches $[10,28,45,76$, $92,115]$, where the individual values are replaced by values computed on small aggregates prior to publication. In other words, instead of releasing the actual values of the individual records, the system releases the mean of the "group" (or any other measure of central tendency, i.e., median, mode, weighted average [115]) to which the observation belongs. The confidentiality of the individual data subjects is protected by ensuring that each group has at least a minimum number of observations, $k$ [54]. If this were not the case, the average would not avoid disclosure because an individual contributing to the partition/ group, or an external individual, can guess the value of another respondent $[22,131]$. The objective of this technique is to group similar records together, so that the replacement of actual values by the means of their associated groups, results in minimizing the $I L[38,74,84]$.

Basically, MATs can be operationally defined with respect to two dominant successive phases $[54,131]$ :

\section{Partitioning}

The original micro-data file is partitioned into several disjointed clusters/groups so that all records in the same group are similar to each other and, simultaneously, dissimilar to the records in other groups. Additionally, each group is forced to contain at least $k$ records.

\section{Aggregation}

This phase computes a certain kind of prototype for each cluster/group, and it replaces the original values in the micro-units by the computed prototype. This phase usually depends on the type of the variable concerned.

The actual implementation of the $M A T$ requires a clustering method and an aggregation method. Such methods were originally used for numerical data types. In this Thesis, we will concentrate on classes of numerical MATs, and will define the Micro-Aggregation Problem, based on such numerical variables. 


\subsubsection{Micro-Aggregation Problem (MAP)}

The $M A P$, as formulated in $[22,45,74,84,92]$, can be stated as follows: A microdata set $\mathcal{U}=\left\{U_{1}, U_{2}, \ldots, U_{n}\right\}$ is specified in terms of the $n$ "individuals", namely the $U_{i}^{\prime} s$, each representing a data vector whose components are $p$ continuous variables. Each data vector can be viewed as $U_{i}=\left[u_{i 1}, u_{i 2}, \ldots, u_{i p}\right]^{T}$, where $u_{i j}$ specifies the value of the $j^{\text {th }}$ variable in the $i^{\text {th }}$ data vector, and it represents the $j^{\text {th }}$ dimension in the space. Associated with the problem is a positive integer, $k$, referred to as the security parameter. Micro-aggregation involves partitioning the $n$ data vectors into $m$ groups so as to obtain a $k$-partition $\mathbb{P}_{k}=\left\{G_{i} \mid 1 \leq i \leq m\right\}$, such that each group, $G_{i}$, of size, $n_{i}$, contains either $k$ data vectors (fixed-size case), or between $k$ and $2 k-1$ data vectors (data-oriented case). Each data vector is contained in exactly one group, implying that $\mathbb{U}=\bigcup_{i=1}^{m} G_{i}$ and $G_{i} \cap G_{j}=\emptyset$. The $j^{\text {th }}$ data vector in the $i^{\text {th }}$ group is denoted by $X_{i j}$ (where each $X_{i j}$ is an element of $\mathcal{U}$ ), while $\bar{X}_{i}$ is the average of the data vectors over the $i^{t h}$ group, and $\bar{X}$ is the average of the data vectors over the entire set of all the $n$ elements of $\mathcal{U}$. Thus, $\bar{X}_{i}=\frac{1}{n_{i}} \sum_{j=1}^{n_{i}} X_{i j}$, and $\bar{X}=\frac{1}{n} \sum_{i=1}^{n} X_{i}$.

The optimal $k$-partition, $\mathbb{P}_{k}^{*}$, is defined to be the one that maximizes the withingroup homogeneity ${ }^{6}$, since a larger value of this index implies a smaller information loss. The within-group similarity is defined as the Sum of Squares Error, $(S S E)$, computed on the basis of the Euclidean distances of each individual data vector $X_{i j}$ to the centroid $\bar{X}_{i}$ of the group to which it belongs. It is given by:

$$
S S E=\sum_{i=1}^{m} \sum_{j=1}^{n_{i}}\left(X_{i j}-\bar{X}_{i}\right)^{T}\left(X_{i j}-\bar{X}_{i}\right)
$$

Analogously, the between-groups similarity is defined as the Sum of Squares Among the groups, $(S S A)$, which is the squared deviations of the means from the total mean.

\footnotetext{
${ }^{6} \mathrm{~A}$ large number of measures which quantify the "group homogeneity" have been reported in the literature. There are usually based on several distance definitions, such as the Euclidean distance, the Minkowski distance, and the Chebyshev distance. The most common homogeneity measure for clustering is the within-group sum of squares, the SSE [52].
} 
It is given as:

$$
S S A=\sum_{i=1}^{m} n_{i}\left(\bar{X}_{i}-\bar{X}\right)^{T}\left(\bar{X}_{i}-\bar{X}\right)
$$

The Total Sum of Squares is denoted by $S S T=S S A+S S E$, or explicitly

$$
S S T=\sum_{i=1}^{m} \sum_{j=1}^{n_{i}}\left(X_{i j}-\bar{X}\right)^{T}\left(X_{i j}-\bar{X}\right)
$$

The Information Loss is quantified by a measure, $I L$ standardized in $[0,1]$ as:

$$
I L=\frac{S S E}{S S T}
$$

The Information contained in the micro-aggregated data is given by the ratio:

$$
\frac{S S A}{S S T}=1-I L
$$

It is important to highlight that the analysis of variance methods can be used as alternative methods to investigate the degree of information that is retained, or, equivalently, that is lost, by using the $M A T$. More details regarding this metric can be found in [115].

\subsubsection{State-of-Art: MATs}

The principle of the $M A T$ is based on the concepts related to the confidentiality of tabular data, where any cell of a table that contains data relative to less than three units, or which is dominated by a single unit, should not be disseminated so as to be consistent with a confidentiality rule. This principle is applied to a micro-data file by aggregating a fixed number of micro-units to avoid being dominated by any one of 
them. Therefore, an $M A T$ proposes that individual micro-units are replaced by the average of a subset of them $[31,32,142]$.

Basically, an $M A T$ relies on a clustering technique and an aggregation technique: $M A T s$ were originally used for numerical data [22, 131], and they can be further classified as Uni-variate methods or Multi-variate methods [28, 91].

\subsubsection{Uni-Variate MATs}

Practical heuristic-based $M A T s$ have been proposed in $[8,22,31,33,34]$. The partitioning mechanism advocated (for uni-dimensional data) in all these papers is the same: First of all, the elements are ranked in an ascending or descending order. Subsequently, groups of $k$ consecutive values assumed by the variable in question are replaced by their average. If the total number of elements $n$ is not a multiple of $k$, the last group will contain more than $k$ elements $[89,115]$. As pointed out in [45], one method to substantially reduce the information loss with such a philosophy is to assign the additional elements to the group containing the modal (or median value [46]) value of the data (rather than including them in the extreme sets).

Two main approaches are used to sort one-dimensional data $[31,45,89,91,115]$ :

\section{Single axis}

The micro-data set being micro-aggregated consists of a single variable or a number of variables. It is called a uni-variate micro-aggregation because the ranking of the micro-data file is based on its projection along a single chosen axis. This approach is recommended when there is a high correlation between the variables, because any single variable will reflect the characteristics of the data vector $[92,115]$. If a particular variable is chosen to rank all the micro-units, this variable must somehow reflect the size of the data vector [91]. The result usually depends on the variable chosen, because it typifies the underlying structure of the population, and, therefore, it could render any statistical inference to be ambiguous [115]. 
Since there is no guarantee that proximity on one variable implies proximity on others, a principle component has been used as a natural alternative to sort the data vector $[31,34,89,91]$. Generally speaking, a principle component analysis reduces the dimensionality of the problem from $P$ to usually 1,2 , or 3 dimensions, by taking into account all the variables of the initial set, and, simultaneously, preserving the underlying multi-dimensional correlation structure. Although it is true that such a scheme is sub-optimal, it can be seen that the resulting solution will not be far from the optimal one because it seeks to minimize the differences between the distances of the elements before and after the transformation process [115]. The rationale for this process is to preserve, as much as possible, the total variance of the original variables in the projected data [100].

Another alternative strategy, like the principle components analysis, takes all the variables into account, and is based on the sum of $z$-scores. Such a criterion gives equal importance to the variables by adding (across all variables) the standardized values for each observation $[31,34,89,91]$. In other words, the rationale for this process is to store the records so as to take into account the variances of all the variables [100]. Finally, it is important to highlight that there is no guarantee that the axis chosen will provide an optimal microaggregation, since these methods are very sensitive to the set of variables chosen [115]. Therefore, a single axis MAT suffers from two sources of $I L$ : The onedimensional data sorting loss and the associated micro-aggregation loss [91, 92]. The authors of [100] have recently replaced the use of projection methods in micro-aggregation by the Sugeno integral aggregation function to calculate the projected axis. They have reported that using any aggregation function reduces the execution time, and, in some sense, increases the data protection.

\section{Individual Ranking}

There are many cases where the variables are not highly correlated. This forces heterogencous values to become members of the same group if any method involving a single axis is used [115]. In order to avoid the $I L$ caused by sorting 
the micro-data file onto a single axis, the authors of $[31,32]$ propose that various uni-dimensional variables should be aggregated separately. This approach, which is known as blurring $[32,33,34]$, deals with multi-variate data sets by micro-aggregating the variables one variable at a time. Indeed, the variables are sequentially and independently micro-aggregated, and every aggregation involves a fixed size of contiguous values. Therefore, this method produces better outlying observations, while leaving the majority of the data structure intact $[31,33]$. The authors of [10] give a quantitative theoretical measure, which estimates the loss of the variance due to individual ranking micro-aggregation methods. Their results show that the expected total variance loss decreases with respect to the increase in the size of the sample under three different distributions using the moments and spacing approaches [115]. Generally speaking, although individual ranking owes its popularity to its simplicity and to the fact that it leads to a low information loss [46], it also leads to a high disclosure risk $^{7}$. It has a conceptual drawback, namely, that it does not partition the $n$ data vectors in the micro-data set on a data-vector basis. Rather, the partition is obtained for each variable in turn, in the micro-data set $[46,89,92]$.

The authors of $[31,46,85]$ highlight an avenue by which the individual ranking $M A T$ can be extended to permit the simultaneous micro-aggregation of several variables, so that a single partition for the entire data set is obtained. An alternative to the individual ranking scheme is the weighted moving average method that requires choosing a number of weights. For example, if we assume that a triple weight $(a, b, c)$ has been chosen, this means that the observations are first ranked independently, and instead of replacing the individual units by the average value, each value for the ranked variables in the observation is replaced by a new computed value comprising of $a \%$ of the previous value, $b \%$ of the current corresponding value, and $c \%$ of the next value in the observation sequence [115]. While this method does not lead to any aggregate being repeated

\footnotetext{
${ }^{7}$ In individual sorting, an intruder knows that the real value of a variable in a data vector in the $i^{\text {th }}$ group is between the average values of the $i-1^{\text {th }}$ group and $i+1^{t h}$ group. Consequently, if these two averages are close to each other, the intruder can determine a narrow real-valued interval to enable him to compromise the security of the system $[46,89,91]$.
} 
$k$ times in each group, it simultaneously suffers from the increment in the $I L$ due to the two missing weighted moving averages at the beginning and at the end. This is because the replacement of these observations, using repetitions of existing values, may lead to values which are far from the original data set [115].

Hanani, a formal algorithm to find the optimal solution for the $k$-partition problem to minimize the $I L$, was proposed by Defays and Nanopoulos in [34]. The paper incorporated the concept of determining a suitable set of hyperplanes separating the $n$ data vectors into a number of homogenous groups. It did this by enforcing the fixed size group and by deriving each partition from a simple ranking of units in terms of a uni-dimensional variable that groups contiguous units [115]. As pointed out in [34], the problem is quite complex, and its practical implementation is both difficult and complicated. For example, it is highly recommended to start the algorithm from different initial partitions in order to avoid falling into local minima [115].

Initial research in the field proposed "fixed" MATs, which required that the size of each partition group was a fixed constant, $k$. These, in turn, led to the Fixed-Size Micro-aggregation or the Classical Micro-aggregation [33, 34, 115] algorithm. Recent developments $[45,89,91,92,126]$ have concentrated on further reducing the information loss by using variable-sized data-dependent groups, which leads to families of Data-Oriented Micro-aggregation algorithms. The philosophy utilized is that groups need not consist of exactly $k$ data vectors, but of at least $k$ data vectors. They also preserve the natural data aggregate by allowing the group size to be between $k$ and $2 k-1$, depending on the structure of the data, so as to lead to more homogenous groups and to minimize the ultimate information loss [28, 45, 92]. Although methods yielding variable-sized groups are marginally more complex than those involving a fixed-size micro-aggregation, they are less likely to compromise the "privacy" of the micro-data sets as shown in [85].

Figure 2.1 illustrates the difference between fixed-size and variable-size $M A T s$, 
where Figure(a) shows nine micro-data units of two variables. If a fixed-size microaggregation with $k=3$ is used, as in Figure 2.1(b), a partition of three groups will be obtained, which seems to be unnatural to the distribution of the data. On the other hand, if a variable-size micro-aggregation is used, as in Figure 2.1(c), a partition of two homogeneous groups will be obtained. This definitely achieves a smaller $I L$ compared to the fixed-size micro-aggregation $[22,46,54,89,91,92,115]$.

Two alternative heuristic approaches that incorporate variable-size micro-aggregation have been presented in $[43,45,91]$ :

- Micro-aggregation based on a genetic algorithm.

- Micro-aggregation based on the Ward's algorithm.

The authors of [45] presented a genetic algorithm that appears as an alternative linear heuristic. It presents the $k$-partitions as a binary string (the "chromosomes"), and combines directed and random search strategies to attain a global optimum $[89,91]$. It starts from a random population of strings, evaluation, selection, crossover, and mutation operators are successively and iteratively applied to obtain a new superior generation that satisfies the convergence criterion. This method offers a good trade-off between the required speed and the information loss when the data set is very large or when the MAT has to be invoked online. But there does not appear to be a theoretical framework that allows optimal values to be found for the five input parameters: The probability of crossover, the probability of mutation, the population size, the maximum number of gencrations, and the convergence criterion used as a stopping iteration. Another weak point is that since this scheme is based on a heuristic random search, it provides solutions that are not reproducible. In other words, running a micro-aggregation based on a genetic algorithm twice on the same data set will probably yicld two different micro-aggregated files [45, 91, 92]. Unfortunately, the technique presented in $[45,91,92]$ is not easily adapted for the the multi-variate case. The main problem comes from the fact that a multi-dimensional space is only partially ordered. This renders the solution to the problem of properly representing 


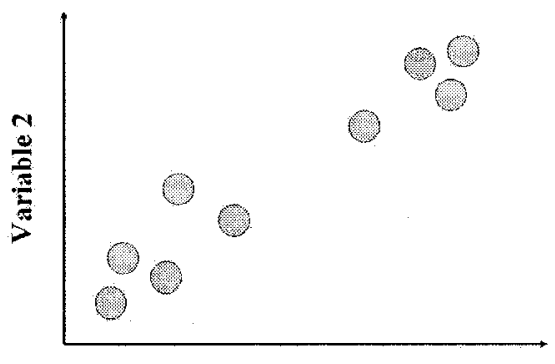

Variable 1

(a)

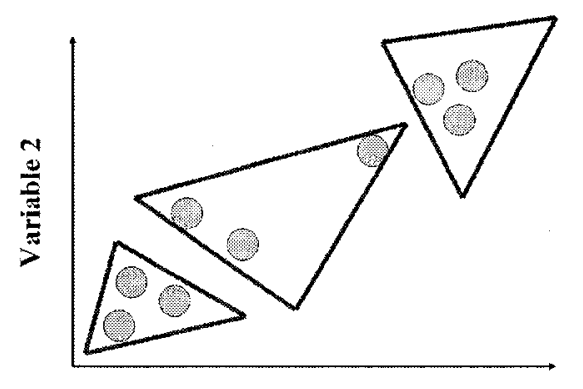

Variable 1

(b)

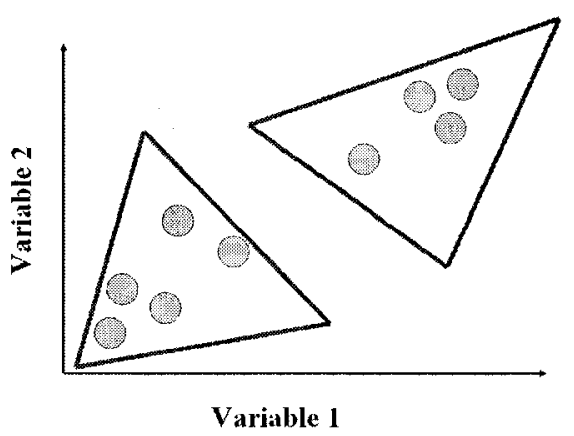

(c)

Figure 2.1: Fixed-size groups versus variable-sized groups. 
multi-variate $k$-partitions as binary strings, to be far from obvious $[45,89]$.

A hierarchical classification method can be used to obtain building blocks for the heuristic MAT so as to yield variable-sized groups. Ward's method, proposed in [139], is attractive because it is stepwise optimal: Two groups or data elements coalesced at each step are chosen so that the increase in the within-groups index, the SSE, caused by their union is minimal. However, Ward's method had to be adapted into the so-called $k$-Ward's method to render it applicable for micro-aggregation [43, 91]. The standard method merely involved building up a grouping hierarchy, whereas the ultimate goal is a $k$-partition of the initial data set. As well, the authors of [43] showed that a $k$-partition algorithm can be naturally turned into a truly multi-variate $M A T$. The main drawback of this method is its storage and running time complexities. Extending this result, a Secure- $k$ Ward scheme was proposed in [85] so as to enhance the individual's privacy. This was done by employing two phases, an intra-group optimization and an inter-group optimization, with the intention of minimizing the information loss and the overall mean deviation. Finally, it should also be mentioned that the scheme proposed in [85] is compatible with the previous $k$-Ward's method proposed in the literature.

The author of [121] showed that the width of the cluster is a suitable measure, especially for the highly skewed data. The total within-cluster width is measured by summing the gap between adjacent members of the clusters, which implies that the size of the cluster is controlled by a minimum number of adjacent gaps. Two direct methods based on the width of the cluster were proposed in $[121,122]$ to microaggregate uni-variate data. First of all, after the data is sorted, all the gaps between the sorted data are computed, as well as the midpoints of the gaps. After this, a binary sorting tree is constructed in which the gap midpoints serve as the internal nodes of the tree and the data values are the external leaves of the tree. Finally, both methods attempt to achieve the micro-aggregation. The first proposed method uses the modified quick sort algorithm, which operates by recursively partitioning its input, while the second method treats the micro-aggregation problem as an optimization problem, which requires an objective function to choose between various sets of 
micro-aggregations and a model with constraints that are included so as to define these possible sets of micro-aggregation. It is important to note that the adjacency concept is simple, natural and easy to implement in the uni-variate data. However, for bi-variate or multi-variate data, the adjacency is natural, the simplicity, and ease of implementation are lost due to not having well-defined multi-variate sorting techniques. Standard techniques from computational geometry have been adapted to the MAP [122], but we consider this to be outside the scope of this Thesis.

The exact solution for the uni-variate $M A P$ that appears in both the individual ranking and projected data approaches has recently been shown to be polynomially solvable as a shortest path problem [74]. Here, optimal partitions were shown to correspond to the shortest path on a graph. The strategy works as follows: First of all, the uni-variate data set is sorted in an ascending order. Thereafter, a graph is constructed in which each arc corresponds to a possible group that may be part of an optimal partition. Each arc is assigned a weight that is the error that would result if that group were to be a part of the ultimate partition. The micro-aggregated solution involves determining the set of arcs in the shortest paths of the graph. The complexity of the resulting algorithm is $O(n \log n)$, where $n$ is the number of records in the database. In spite of having a polynomial algorithm for the optimal univariate $M A P$ [74], heuristic algorithms are needed, because attaining the minimum information loss for the $M A P$ is NP-hard for the multi-variate case [84, 103].

Finally, Table 2.3 summarizes the main numerical uni-variate micro-aggregation methods and their classification.

\subsubsection{Multi-Variate MATs}

A large number of heuristic methods have been proposed for solving the multi-variate $M A P$. These methods can be further summarized as follows [28, 46]:

\section{Projecting multi-variate data onto a single axis}


Table 2.3: The classification of the numerical uni-variate micro-aggregation methods.

\begin{tabular}{|c|c|}
\hline Classification & Micro-Aggregation Methods \\
\hline \multirow{4}{*}{ Single axis } & Particular variable \\
\hline & Principal component \\
\hline & Sum $z$ score \\
\hline & Aggregate function \\
\hline \multirow[t]{2}{*}{ Individual methods } & Individual ranking \\
\hline & Weighted moving average \\
\hline \multirow{5}{*}{ Heuristic partitioning technique } & Hanani's algorithm -(variance) \\
\hline & $k$ Wards -(distance) \\
\hline & Genetic algorithm \\
\hline & $\begin{array}{l}\text { Minimize the total within cluster width } \\
\text { (Quick Sorting or optimization approach) }\end{array}$ \\
\hline & Shortest path -(Optimal) \\
\hline
\end{tabular}

Instead of using a multi-dimensional distance to sort the data vectors, all practical methods perform a straightforward uni-dimensional sorting by projecting the former onto a single axis (i.e., using the first principle component, the sum of $z$-scores or even a particular variable) $[91,92 \mid$. It is necessary to stress that, all the above described numerical uni-variate $M A T s$ can easily be extended to multi-variate $M A T s$ using any projection method.

\section{Unprojected multi-variate data}

A natural improvement of the above is to deal directly with multi-variate data without projection. Either the data vectors can be sorted based on comparing all the variables simultaneously or they can be independently sorted them using more than one (for example, two or three) variable at a time. A number of heuristic methods to micro-aggregate unprojected raw data utilize search techniques including hierarchical clustering, genetic algorithms, and tabu search $[45,92]$.

In spite of the simplicity of the uni-variate micro-aggregation and its low complexity, it is not very attractive because it either suffers from a high $I L$ due to the data transformation (projection on to single axis) or from high $D R$, (especially, for 
the individual ranking) $[46,45,89]$. The empirical results reported in most of the papers suggest that the multi-variate micro-aggregation on unprojected data offers a superior trade-off between the $D R$ and $I L$, especially when groups of three or four variables are micro-aggregated at a time, rather than all variables at the same time $[44,45,46]$.

The first algorithm to accomplish this was invented in 1998 by Domingo-Ferrer et al. $[91,45]$ and called the "Multi-variate fixed-size micro-aggregation" method. It micro-aggregates the multi-variate micro-data file based on the concept of the diameter distance of the data set as follows: It first searches for the two most distant records from the data set, say records $r$ and $s$. Using these, two clusters are created; the first one comprises of $r$ and its (k-1) nearest records, while the second comprises of $s$ and its (k-1) nearest records. If there are at least $2 k$ records that do not belong to the two clusters formed, the aggregated records are then computed for these two clusters, and they are removed from the original data set. This process is iteratively repeated until all the records in the original data set have been used in generating the new micro-aggregated file or, in other words, until the original data set is empty. But if there are between $k$ and $2 k-1$ records, which do not belong to the two clusters formed, we form a third cluster with those records and stop the process. In a case when we have less than $k$ records that do not belong to the two formed clusters $r$ and $s$, it is mandatory to add these remaining records to the closest cluster $[45,52,91]$.

The problem encountered is one of determining how to decide which are the most distant records. A truly multi-variate criterion has to be followed instead of projecting the data onto a single axis ${ }^{8}$. The most distant records are defined according to a truly multi-variate sorting criterion, which is the distance matrix, and this is referred to as the "Maximum-distance" $(M D)[45,89,91,92,115]$ scheme. One of the disadvantages of using this sorting critcrion is related to the storage capacity, since it requires quadratic time (quadratic in the size of the data set) [89]. Solanas et al. have

\footnotetext{
${ }^{8}$ If a single axis is used to make a decision of the two most distant records in the multi-variate fixed-size micro-aggregation algorithm, then this algorithm is equivalent to performing a uni-variate fixed micro-aggregation.
} 
recently shown that when the number of records is very large, the distance matrix can be efficiently stored by applying the blocking technique [127]. Another small disadvantage is that the grouping resulting from the $M D$ usually depends on the extreme data vector with which one starts. In other words, the efficiency of the solution depends on which extreme data vector is taken as the first data vector and which is taken as the last $[45,89,91,92,115]$. This concern is clearly illustrated in Figure 2.2. If $k$ is set to equal 3, Figure 2.2(a) represents a micro-data file consisting of 6 points with 2 variables. In this case it is obvious that the most distant records are $a$ and $e$. Starting from vector $a$ we will have two clusters, shown in Figure 2.2(b). But if we choose to start from vector $e$, we will have another different two clusters, as shown in Figure 2.2(c). Typically, the differences in the $I L$ that result from choosing either extreme vectors as the first or last are small $[45,89,91,92,115]$.

A natural way to obtain multi-variate data-oriented methods is to generalize some of the uni-variate data-oriented methods described in Section 2.6.3.1. The strongest advantage of a $k$-Ward $M A T$ is that it is easily adapted to the case of multi-variate unprojected data. The reason for this is that the $k$-Ward method was originally designed as a multi-variate clustering algorithm $[62,79,91,139]$. It is important to remind the reader that projecting the multi-variate data in order to know the first$k$ and the last- $k$ elements in the $k$-Wards algorithm, (as will be described in greater detail in Chapter 3), is not equivalent to a data-oriented uni-variate micro-aggregation scheme on projected data $[45,89,91,92,115]$.

In 2005, an enhanced version of the "multi-variate fixed-size micro-aggregation" method, called the Maximum Distance to Average Vector (MDAV) scheme, appeared in [60] and was implemented as a built-in technique in the $\mu$-ARGUS Software tool version 4.0 [77]. This modification is based on the concepts used to compute the micro-aggregated record, namely, it depends on computing the centroid of the data set, instead of computing its diameter. After this, a quick search for the most distant record from the centroid, say $r$, is done. Subsequently, a new search for the most distant record from the record $r$, say $s$, is accomplished. The next step consists of creating two clusters, the first one comprising of $r$ and its $k-1$ nearest records, while 


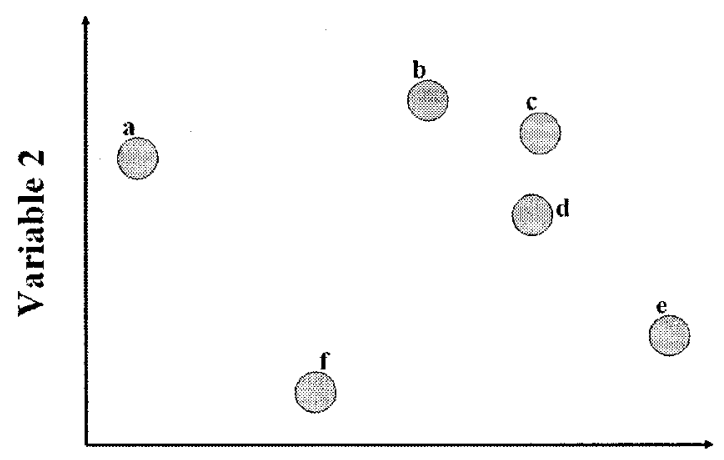

Variable 1

(a)

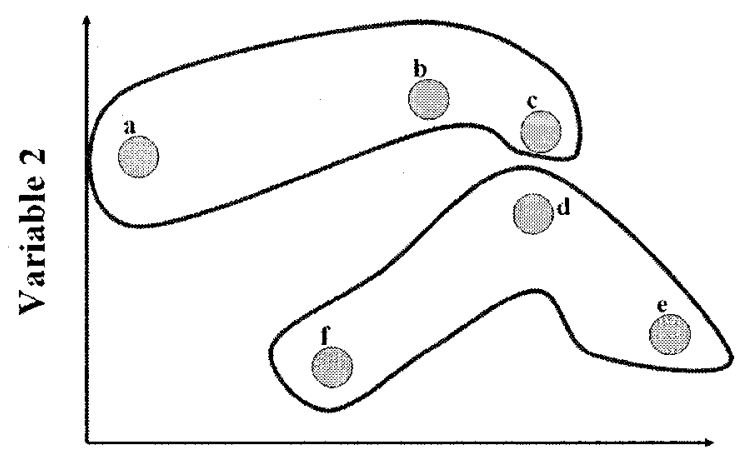

Variable 1

(b)

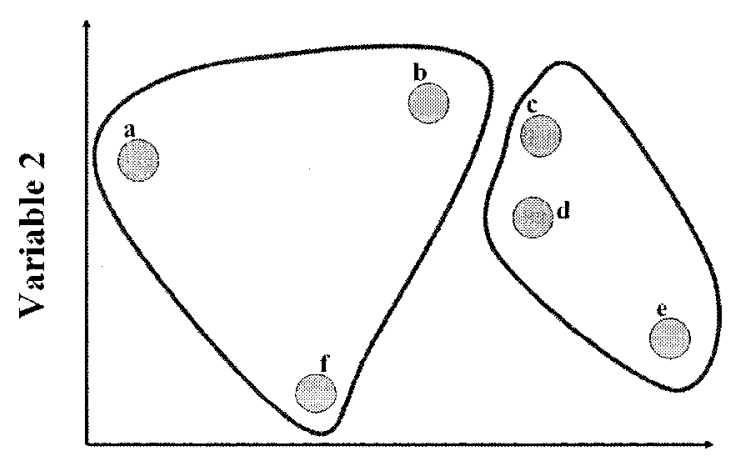

Variable 1

(c)

Figure 2.2: Grouping with the maximum distance criterion. 
the second comprises of $s$ with its nearest $k-1$ records. At the end of this stage, the two clusters are micro-aggregated and removed from the original data set. The latter steps are iteratively repeated until no more records remain in the original data set. The advantages of this new modified version of the $M D A V$ are the increased speed of the micro-aggregation and the resulting reduction of the $I L[52]$.

$V-M D A V$, which stands for Variable-size Maximum Distance to Average Vector, is the first heuristic method applicable for a multi-variate variable-size microaggregation [126]. It improves the well-known $M D A V$ method in terms of lowering the $S S E$ and, simultaneously, maintains an equivalent computational cost. $V-M D A V$ follows a strategy similar to the $M D A V$ method, by building a distance matrix and computing the global centroid. Thereafter, once the most distant record from the global centroid is found, a group of $k$ records is formed by selecting the $k-1$ records closest to the initial one. The next step is the most important one, where the $V-M D A V$ overcomes the fixed-size constraint of the previous heuristics by adapting the data set distribution and generating variable-size groups. This step is achieved by searching for the closest unsigned record in the last formed cluster, $e_{\text {min }}$, and computing its distance, $d_{i n}$. After this, we compute the minimum distance from the selected record $e_{\text {min }}$ to any of the remaining unsigned records, $d_{\text {out }}$. A decision of the inclusion of $e_{\min }$ into the cluster is made if, and only if, $d_{i n}<\gamma d_{\text {out }}$, where $\gamma$ is a gain factor; otherwise $e_{\min }$ will not join the cluster. The extension step is terminated when either the group size is equal to $2 k-1$ or when a decision of inclusion is not satisfied. The entire procedure is iteratively repeated until all the records in the original data set have been used in generating the new micro-aggregated file $[52,126]$.

The computation of the Minimum Spanning Tree (MST) leads to another multivariate $M A T$ [84]. This yields a new clustering algorithm obtained by splitting the minimum spanning tree using a constraint on the minimum group size. The $M S T$ can be considered as a potential strategy for any practical application, since it exploits the natural clustering effects in the data that are consistent with the objective of reducing the $I L$. 
Domingo-Ferrer et al. have recently reported a multi-variate micro-aggregation heuristic referred to as the " $\mu$-Approximate" algorithm, which involves an approximation bound for the $S S E[39,51]$. The $S S E$ of the micro-aggregated data is no longer greater than a multiple of the minimum $S S E_{o p t}$, which corresponds to the optimal multi-variate $M A P[102,103]$. Bounds have been proven for two different optimality criteria: An $\bigcirc\left(k^{2}\right)$ - approximation bound for minimum within-groups sums of Euclidean distances, and an $\bigcirc\left(k^{3}\right)$ - approximation bound for minimum within-groups sums of squares [51]. Their philosophy involves creating a directed forest where all the original records are vertices. Each vertex has at most one outgoing edge, i.e., $(u, v)$ is an edge between the two vertices $v$ and $u$ if $v$ is one of the $k-1$ nearest neighbors of $u$. The size of every tree in the forest should be between $k$ and $2 k-1$. When the algorithm terminates, the vertices in each tree are replaced by their aggregation [39]. The authors of [51] also explain how the value of $k$ can be determined.

As opposed to the above, the authors of [49] present a tighter approximation for the special case when $k=2$, which is called the "Polynomial-time 2-approximation" for multi-variate micro-aggregation. Their solution represents an interesting option to implement 2-anonymity, because it results in low $I L$ and thus high data utility in most scenarios except when internal intruders are likely, and when outliers are exist.

A more recent hybrid technique for multi-variate micro-aggregation combines a multi- variate heuristic yielding fixed-size groups and a genetic algorithm yielding variable-sized group [88]. It is well-known that fixed-size heuristics are fast and able to deal with large data sets. On the other hand, the genetic algorithm obtains very good results (i.e., optimal or near-optimal), although it can only cope with very small data sets. Consequently, this new mixture blends the advantages of both types of heuristics and avoids their shortcoming. The main idea behind this hybrid algorithm is to partition the data set into a number of groups by invoking any fixed-size multivariate micro-aggregation such as the $M D A V$, and then optimizing these partitions by means of the genetic algorithm [88]. 
An adaptive fuzzy $c$-means based micro-aggregation technique has recently been proposed to build a set of clusters in which elements can belong to more than one cluster at the same time [133]. In other words, the fuzzy $c$-mean leads to a set of nondisjoint clusters where each element has a partial membership index that expresses the degree of its membership to a particular cluster. Generally speaking, the membership degree is specified in the interval $[0,1]$, where 0 implies "no membership" and 1 means a "full membership". The most interesting part of this algorithm involves replacing the individual values by the centroid value of each cluster. This is achieved by selecting a cluster to which certain records belong and by further selecting the values of different variables in the same records. There are, thus, four different alternative methods for the aggregation stage $[57,134]$ :

- Selecting a cluster for a given record

The selection of a cluster is based on either a uniform probability distribution over the clusters with a non-zero membership or a probability distribution proportional to the membership degree.

- Selecting the values for different variables in the same records

Once the cluster is selected, either all variables are aggregated using the same cluster centroid or a cluster selection technique is applied for each variable of the same record.

The authors of $[57,134]$ report that the fuzzy $c$-means method performs better than an additive noise, data distortion scheme that uses a probability distribution followed by resampling. However, it performs worse than the classical micro-aggregation, although with respect to the $D R$ criterion, it is better. The reason behind this is that this method does not yield any clue about the masking method used nor about the clusters obtained by the method [57, 133]. Recently, an alternative fuzzy clustering method was proposed in [134], referred to as an entropy-based fuzzy $c$-means method. The only difference between the latter version and the previous one is the technique by which fuzziness is introduced. The fuzzy $c$-means strategy produces a fuzzy solution by involving the means, while the latter uses a term based on the entropy to force a 
fuzzy solution. It appears as if an entropy-based clustering algorithm is significantly better than the standard fuzzy $c$-mean based clustering algorithm, additionally it is more stable [134].

It is important to remark that the exact solution to the $M A P$ in the multi-variate case, (without projecting the data onto an "Euclidean" space of dimension of two or greater), is shown to be NP-hard $[102,103]$. This opens an avenue of research, namely to produce an approximation polynomial time technique that can out-perform all the other techniques. This problem remains open.

We conclude this section by presenting in Table 2.4 a summary of the main numerical multi-variate micro-aggregation methods and their classification.

Table 2.4: The classification of the numerical multi-variate micro-aggregation methods.

\begin{tabular}{|l|l|}
\hline \multicolumn{1}{|c|}{ Classification } & \multicolumn{1}{|c|}{ Micro-Aggregation Methods } \\
\hline \hline \multirow{4}{*}{$\begin{array}{l}\text { Projection onto single axis by } \\
\text { using principle component, } \\
\text { sum of } z \text {-scores, or even } \\
\text { particular variable }\end{array}$} & Individual ranking \\
\cline { 2 - 2 } & Weighted moving average \\
\cline { 2 - 2 } & Hanani's algorithm \\
\cline { 2 - 2 } & Genetic algorithm \\
\cline { 2 - 3 } & K Wards \\
\cline { 2 - 2 } & Shortest path \\
\hline \multirow{5}{*}{ Unprojected methods } & Multi-variate fixed-size micro-aggregation \\
\cline { 2 - 2 } & Minimum Spanning Tree (MST) \\
\cline { 2 - 2 } & Maximum Distance to Average Vector (MDAV) \\
\cline { 2 - 2 } & Variable-size Maximum Distance to Average Vector $(V-M D A V)$ \\
\cline { 2 - 2 } & Fuzzy C-mean \\
\cline { 2 - 2 } & $\mu$-Approximate Algorithm \\
\cline { 2 - 2 } & Hybrid Algorithm \\
\hline
\end{tabular}




\subsubsection{Categorical MATs}

Quantitative micro-aggregation has been a research topic for more than ten years, while qualitative micro-aggregation is quite new [54]. Although the actual implementation of micro-aggregation requires only a clustering method and an aggregation procedure, the reason behind this relative lag in the latter field is the difficulty of involving both these processes in the case of categorical data [60,131]. A fair bit of attention has, however, recently been given to extending the $M A T$ to categorical data types [56, 58, 115, 131].

The aggregation function for categorical data can be distinguished on the basis of the categorical data type. In other words, the main operator in this case is the plurality rule (mode, or the voting procedure) for the nominal scales, while for the ordinal scale the median is used for the aggregation step $[22,54,56,60]$.

The author of [33] was the first one to suggest a generic method based on the individual ranking technique for multi-variate data. The subsequent clustering method for categorical data appeared in [131], and this was, in turn, based on the $k$-modes algorithm inspired by the $k$-means algorithm for numerical data. More recently, the $M D A V$ scheme has also been extended for categorical data $|60|$.

As we will not be discussing categorical data in this Thesis, our review of this sub-area terminates here.

\subsubsection{Additional Applications}

Apart from being methods in their own right that permit micro-aggregation to be used as an $S D C$ technique to yield a good trade-off between the $I L$ and $D R$, the $M A T$ can also be used in other applications, such as in linear regression and $k$-Anonymity.

The authors of [66] enhanced the classical multiple linear regression model, which obtains the best linear unbiased estimates of the regression coefficients, to render it 
more secure. This was achieved by deriving the estimator from the grouped aggregated data, although partial aggregation usually results in the loss of efficiency for estimates of the regression coefficients and their residual error variance. But this loss can be minimized by generating groups that are homogeneous with respect to the independent variables being analyzed. In other words, this can be done by minimizing the within-group variation and by maximizing the between-group variation.

Micro-aggregation has also been found to be an interesting alternative to generalization or suppression for $k$-anonymity ${ }^{9}[5,39,60]$. A solution to $k$-anonymity is based on generalization and suppression techniques. The latter perform poorly in terms of data quality because they are not suitable for all types of attributes [39]. The authors of $[39,60]$ report that using micro-aggregation for $k$-anonymity circumvents most of the problems of generalization/suppression techniques because micro-aggregation is a unified approach, unlike the dual method that combines generalization and suppression. Although the optimal $M A P$ is $N P$-hard, many heuristic approaches lead to a solution that is close to the optimal one. Micro-aggregation does not complicate the data analysis by introducing new categories, or by suppressing certain data fields. Finally, micro-aggregation is also useful in protecting fields containing continuous data because it does not harm or destroy the underlying numerical semantics [60]. The author of [41] extends the use of micro-aggregation of all attributes for $k$-anonymity to implement the property of $P$-sensitive $k$-anonymity in a more unified and less disruptive way.

Micro-aggregation has also been reported to be relevant to the field of Artificial Intelligence $(A I)$, and has been used in some applications that require increasing the knowledge of a system for decision making and domain representation [59]. It can also be used in data mining or to compress the data set while minimizes the $I L[52]$.

\footnotetext{
${ }^{9} k$-anonymity is a useful concept to solve the tension between the data utility and respondent privacy in individual data. An anonymized data set is said to satisfy $k$-anonymity for $k>1$, if, for each combination of the values of a key identifier, at least $k$ records exist in the data set sharing that combination $[39,60]$.
} 


\subsubsection{Properties and Characteristics of the MAT}

\subsubsection{Variants of the Methods}

Micro-aggregation performs quite well with respect to the different existing criteria for $I L$ and $D R$. Moreover, the authors of $[53,57,131,133,134]$ show that it is the second-best method for numerical data, after the rank swapping method ${ }^{10}$. The authors of [94] show that the choice of parameter in the MAT has less influential than in rank swapping. Therefore, the behavior for micro-aggregation is more robust, even through medium and high values. It is important to mention that both $M A T$ and rank swapping offer more protection to outliers than other SDC techniques. It is necessary to note that there are some important issues that should be taken into consideration when micro-aggregating social or business data. These issues are discussed below.

- Selecting a segmentation of the set of the variables to apply the micro-aggregation It is important to determine the number of variables, (the best combination that leads to the minimum value of the $I L[33,34,90])$, that is to be used in the micro-aggregation process. Moreover, the initial vector of variables can be segmented into a number of variables, multi-variate or uni-variate, which we called segments. $[31,32,66,115,119,120]$. A set of $P$ variables can be treated as a single multi-variate variable, resulting in a single grouping, or as $P$ separate variables, resulting in $p$ groupings for each. It is well-known that dealing with all the variables simultaneously minimizes the loss in the information gained. It is reported in [54] that multi-variate micro-aggregation on unprojected data taking two or three variables at a time (rather than all variables) is the microaggregation algorithm offering the best trade-off between $I L$ and $D R$.

- Selecting the number of steps to be applied in the micro-aggregation

\footnotetext{
${ }^{10}$ Authors of $[32,33]$ show that some people reject data swapping on the basis of protecting individual data without preserving the moments of the initial distribution.
} 
It is important to determine the number of steps that are required to microaggregate all micro-units in the original file [66, 115]. For example, let us assume that 7 variables are used to micro-aggregate a micro-data file with 13 variables. The micro-aggregation process can be done either in a single step, by using all the variables together, in two steps, by using 3 variables in the first step and 4 variables in the second step, or in three steps, by using 3 variables in the first step and 2 variables in the second and the third steps. The authors of $[119,120]$ show that the micro-aggregation in more than one step leads to a lower $I L$, and, simultaneously, a higher $D R$ than the usual single-step micro-aggregation technique.

- Selecting the type of the variable to be applied in the micro-aggregation It is usually recommended that independent variables are chosen to microaggregate the micro-units, and it is preferable that the variable chosen is either discrete, real, or ordinal $[32,33,66]$. These types of variables do not take values over a very restricted set, and so they will minimize the probability of being compromised by intruders [14]. Generally speaking, the decision of determining which variables have to be involved in the micro-aggregation process is based on the previous knowledge about the characteristics of each variable $[119,120]$.

- Selecting the degree of aggregation

The number of records per group, which is usually represented by $k$, a predefined threshold, plays an important role in determining the loss in the data utility, as well as the loss in the confidentiality. In fact, increasing the degree of the aggregation will decrease the $I L$ and, simultaneously increase $D R[33,66]$. The minimum size of such groups usually depend on several factors: The rules adapted in some countries, the degree of confidentiality of a segment of the variable, and the values taken by the variables in a group [32]. It is worth mentioning that the decision of imposing a fixed size or a minimal size constraint has a clear effect on the homogeneity of the groups. Generally speaking, a minimal size constraint usually leads to more homogencous groups than the fixed size, and it is more difficult to be compromised $[28,45,85,92,115]$. In practice, 
the statistical law that defines the requirements in order to make the data set confidential, usually sets the value of $k$ to be either 3 or $4[46,115]$. If $k$ is greater than 4, it can be argued that basic analytic properties, such as these required for regression, can be seriously affected [140].

- Selecting the clustering procedure

The methods are mainly characterized by the way in which the homogeneity of the groups is measured [11,32,33]. The main difference between the MATs lies in the way clusters are built (by modifications of a standard technique, novel approaches using genetic algorithms with an appropriate fitness function, etc.) [131]. The authors of [66] have suggested having a built-in function of the researcher's cardinal preference with respect to the grouping criterion. It is worth mentioning that applying the grouping criterion on the projected data will increase $I L$ caused by the micro-aggregation itself $[11,45]$.

- Selecting the aggregation statistical procedure

The MATs aim to partition the whole micro-units into groups of either $k$, or at least $k$ micro-units group size. These micro-units are replaced by certain aggregation statistics, which are either the mean of the group or another measure of central tendency (i.e. median, mode, weighted average) $[31,32,115]$. This usually depends on the type of the variables whether it is quantitative or qualitative [131].

- Selecting the sorting procedures

The combination of fixed-size MATs with sorting algorithms could reveal some confidential information, although the sorting methods have to set up groups that are as homogeneous as possible in order not to lose too much information. Special attention should be paid to ranking the individuals in either an ascending or descending order [115]. This is especially important if some information about an individual is available through a non-confidential source (prior knowledge), because it is easy to gain added confidential information by using successive sorting instructions [66]. A general rule applicable for the sorting strategy used for single axis methods typically depends on the position of the 
majority of the outliers [115]. If the outliers of the variables are to the left of the distribution curve, an ascending order has to be used in ranking the values of the variable. But if they lie to the right side of the distribution curve, a descending order is used to rank the values of the variables.

- Measuring the homogeneity of a group

Since the $S S T$ is fixed for a given data set, one should attempt to find a grouping that minimizes the $S S E[31,32,33,91]$. Moreover, the concept of the underlying similarity in the definition of the groups can lead to various interesting variants of the basic methodology. The quantification of homogeneity in the multivariate data set is not easily defined, because it can be measured in various ways, for example, using the within-group variance, the entropy, or a measure based on any type of distance [31]. For the quantitative variables, the Euclidean distance or the variance will determine the formation of groups, while for the ordinal variable this information will be determined by the absolute value of the difference in rank or by the entropy concept [32].

At this juncture, we mention some of the drawbacks of $M A T s$, which can be listed as follows:

1. The information present in the groups obtained by an $M A T$ may sometimes obscure useful statistical information found in the database.

2. Adding or deleting certain records from different groups is expensive.

3. The bias introduced by using this technique decreases the variance with an amount equal to $\frac{1}{N} \sum_{j=1}^{m} \sum_{i=1}^{k_{j}} \delta_{i j}^{2}$, where $m$ represents the number of groups, $k_{j}$ represents the number of records in groups $j, \delta_{i j}$ is the distance between the centroid of the $j^{\text {th }}$ group and the $i^{\text {th }}$ record which belongs to that group, and $N$ represents the number of records in the micro-data file [104].

Finally, we conclude that there is no single method that out-performs the others [81], since there are no descriptive criteria defined to judge which is the best method 
with respect to the complementary objective ( $I L$ and $D R$ ), the accuracy, and the execution time. Moreover, the choice of an optimal method usually depends on the nature of the data (i.e., the type of the variables and the statistical relation between them) [11].

\subsubsection{Inference Control in Data Mining Versus MATs}

While MATs introduce intentional distortion to protect micro-data sets against disclosure, $A I$ techniques try to overcome the data distortion. Generally speaking, the goals of $A I$ and $M A T$ seem to be contradictory. This is because while MATs are used to hide information, $A I$ techniques are used to infer information. The power of any $A I$ technique is in its ability to reconstruct the original data from several distorted versions [137]. This can be achieved either by [59]:

- Building a model for a given variable so as to discover a relationship between a sensitive attribute and others. Thus, if the attribute is disclosure protected, the aim is to infer the original value.

- Releasing multiple protected versions of the same original data file will definitely increase the disclosure risk. In this case the users will try to reconstruct and find the original information out of $n$ different distorted versions of the same file [137], either by using re-identification procedures based on sharing a set of common variables or by processing non-common variables.

It should be mentioned that $A I$ techniques can be beneficial if the data mining tools are combined with the micro-aggregation software packages. The protection can be achieved in two stages: First of all, the original data is protected by using one of the $S D C$ techniques offered by the package. Secondly, the data mining tool will be used on both the original and the protected data sets to find models that lead to the original sensitive data. If the data mining scheme yields a good approximation, the data protector should return to the first stage and be enhanced. 
Privacy preserving data mining is a related field that possesses similar goals [131]. It involves a set of two or more parties that want to compute joint shared functions on their private inputs. These computations should be done in a secure way, so as to preserve both correctness and privacy [81]. From this perspective, it can be seen that while the $M A P$ is oriented to statistical databases, privacy preserving data mining is oriented to company proprietary information [131]. However, there seems to be large rewards in combining both of these strategies, and this is also another open area for research.

\subsubsection{Clustering Techniques Versus MATs}

The clustering problem and the $M A P$ are similar when it concerns finding an optimal partition of elements for homogeneous groups. But, the clustering problem differs from the classical $M A P$ in two aspects: First of all, in a clustering problem, the number of groups is given a priori, whereas for micro-aggregation it is not. Secondly, and more importantly, in a clustering problem there are no constraints on the cardinalities of the groups, whereas in the $M A P$, each group must contain no fewer than $k$ elements $[45,28,91,84,121]$. Many data clustering algorithms have been proposed in literature $[15,79,130,138]$. Clustering algorithms may be good candidates for $M A T$ if they can be adapted by efficiently enforcing the group size constraint [84]. 


\section{Chapter 3}

\section{Enhancing the Heuristic $k$-Ward MAT}

\subsection{Introduction}

We consider the problem of securing a statistical database by utilizing the well-known micro-aggregation strategy, and in particular, the $k$-Ward strategy introduced in [139] and utilized in [45]. The latter scheme, which demonstrates a good trade-off between $I L$, and $D R$, coalesces the sorted data attribute values into groups, and on being queried, reports the means of the corresponding groups. In this chapter ${ }^{1}$ We demonstrate that such a scheme, as reported in the literature, can be significantly improved on two fronts. First of all, we minimize the computations done in evaluating the between-class distance matrix, to require only a constant number of updating distance computations. Secondly, and more importantly, we propose that the data set be partitioned recursively before a $k$-Ward strategy is invoked, and that the latter

\footnotetext{
${ }^{1}$ A preliminary version of some of the results from this chapter appear in the Proceedings of ACISP'06, the Eleventh Australasian Conference on Information Security and Privacy, in Melbourne, Australia, in July 2006 [65]. The journal version of these results is currently under review.
} 
be invoked on the "primitive" sub-groups which terminate the recursion. Our experimental results, done on uni-variate and projected multi-variate data using two benchmark data sets, demonstrate a marked improvement. While the $I L$ is comparable to the $k$-Ward micro-aggregation technique proposed by Domingo-Ferrer et.al. [45], the computations required to achieve this loss is a fraction of the computations required in the latter - providing a computational advantage which sometimes exceeds $80 \%$ if one method is used by itself, and more than $90 \%$ if both enhancements are invoked simultaneously.

This chapter is organized as follows: The Ward algorithm and $k$-Ward_MAT are described comprehensively in Section 3.2 and Section 3.3, respectively. Section 3.4 presents the improved versions of the $k$-Ward $M A T$, which is followed by an algorithmic description of the two new modifications. Experimental results using the two usual reference benchmark data sets and the related discussions are provided in Section 3.5. Finally, Section 3.6 presents the overall conclusions of this work.

\subsubsection{Contribution of the Chapter}

As mentioned earlier the challenge in micro-aggregation is to modify the original data so that both the risk of disclosing confidential information and the loss of the data utility should be below a certain threshold determined by the data protector $[42,142]$. This means that the data protector is the one who determines the $M A T$ to be used based on the type of the data and its application [142].

The advantage of the shortest-path technique is that it leads to the optimal solution for uni-variate MATs from the perspective of the IL [74]. However, unfortunately, it is often not the optimal solution from the perspective of the $D R$ - since the $I L$ and $D R$ criteria are often conflicting. This is because the shortest-path method provides similar statistics to the original data, which, in turn, increases the likelihood of information being disclosed. The literature $[39,60]$ reports that this problem can be overcome by solely determining the appropriate value of the minimum group size 
parameter. This is achieved by ensuring $k$-anonymity, where each data subject is distinguishable from at least $k-1$ other data subjects. But the reader should observe that approximate heuristic uni-variate $M A T s$ which use a smaller value of $k$ usually provide more protection, in spite of forfeiting some information when compared to the optimal solution. This motivates the present research which seeks for a "fast" scheme which can be effective for both the $I L$ and the $D R$ criteria.

The $k$-Ward is one of the heuristic MATs, which demonstrates a good trade-off between the $I L$ and the $D R$, but it suffers from a fairly high computational complexity, as will be explained presently. The main contribution of this chapter is to render the k-Ward MAT a pragmatically applicable solution, and this is done by increasing the speed of the micro-aggregation process by incorporating two considerations, namely that of invoking recursive computations, (which is crucial for large-sized data sets), and by utilizing only critical values of the so-called distance matrix. This, in turn, is achieved by two enhancements to the $k$-Ward algorithm, namely those which we have called Recursive $k$-Ward $(k W R)$, and the $k$-Ward Diagonal $(k W D)$, respectively. This chapter demonstrates the power of these modifications in increasing the speed of the strategy while almost preserving the $I L$.

It should be mentioned that the strategy of using our recursive method can actually be applied to any known $M A T$. As a proof of concept, we initiate this research by demonstrating its power on uni-variate data. However, the power of such a method can be appeared also for multi-variate data sets by projecting them onto a specific axis in a transformed domain using, for example, a Principle Component Analysis, $(P C A)$, the sum of $Z$-Scores, $(Z S)$, or a particular variable ${ }^{2}$. To be objective and unbiased, though, it is fair to point out that these mechanisms cannot be applied without projecting the multi-variate data set onto a single axis. This is because there is no straightforward formal algorithm to sort multi-variate data without performing an appropriate projection $[45,46,89]$. Furthermore, these schemes critically depend

\footnotetext{
${ }^{2}$ This has also already been done for both the $P C A$ and $Z S$ projection methods and will be reported presently.
} 
on the fact that the data points have to be ordered ${ }^{3}$.

The advantage of these improved versions will be marked especially for "large" data sets. Since the distance matrix involving the set of data vectors cannot be computed and stored a prior $i^{4}$, these distances and the resulting matrix must be computed "on demand"- i.e., as and when they are needed. This, of course, demands a considerable computational overhead [91], which is significantly reduced by using the improved versions of the $k$-Ward's method.

The combination of invoking a recursive computation and requiring a small subset of the distance computations is novel - we are not aware of any comparable results. However, the remarkable reduction in computation (sometimes as much as $80 \%$ ) renders the contribution of the chapter significant.

\subsection{Ward's Method}

The Ward method is an agglomerative clustering method that creates hierarchical groups of mutually exclusive partitions. Each partition attempts to attract members that are maximally similar with respect to certain specified characteristics. Given $n$ vectors, the first partition consists of $m=n$ single-record groups. It then permits their reduction to $m-1$ disjoint partitions by considering the union of all possible $m(m-1) / 2$ pairs and selecting a union having a maximal value for the functional relation that reflects the criteria chosen by the user. This process can be repeated until the last partition consists of a set of groups with an acceptable criterion value

\footnotetext{
${ }^{3}$ The authors of [45] have presented a "multi-variate k-Ward" heuristic that does not rely on such a uni-variate projection. With little work, we believe that our recursive strategy can be rendered applicable to such a methodology. Although we are currently considering how our matrix optimizing operations can be applied to the scheme proposed in [45], it does not seem to be straightforward. This work is still open.

${ }^{4}$ Solanas et al. have recently proposed a strategy by which the distance matrix can be effectively stored when the number of records is very large, by applying the so-called blocking technique [127]. We are currently investigating how our methods can be further enhanced by incorporating such blocking techniques.
} 
[139]. The Ward method is formally described below.

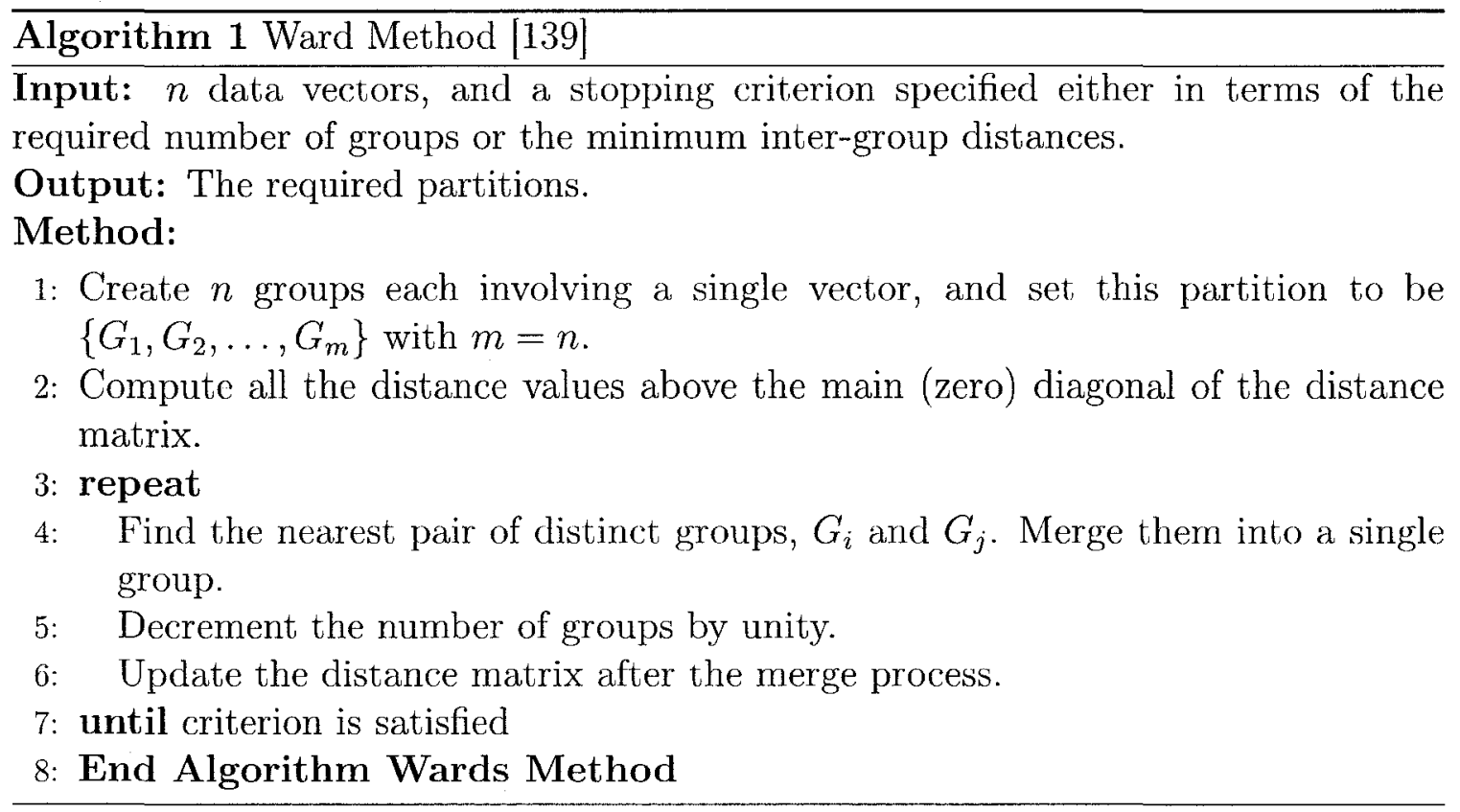

A crucial component of the Ward algorithm is the computation of the so-called distance matrix involving all the groups. Essentially, the latter matrix is a symmetric $m \times m$ matrix, where $m$ is the number of groups at any particular step. The intention is that by computing this matrix we will be able to coalesce two groups, $G_{i}$ and $G_{j}$, resulting in a partitioning with a smaller number of groups, without forfeiting the overall similarity index excessively. Unfortunately, the $k$-Ward algorithm, as it has been used and reported, computes the entire matrix involving all the inter-group distances, which renders the computation excessive. Our goal is to minimize these computations by utilizing the ordered nature of the uni-variate data when data is, in itself, uni-dimensional, and in the projected case when the data is multi-variate, as will be explained presently. 


\section{$3.3 k$-Ward Micro-Aggregation Technique}

Ward's agglomerative hierarchical clustering method, described above $[15,139]$, has been modified to provide an optimal $k$-partition solution by enforcing the group-size constraint $[43,45,91]$. Such a $k$-Ward_MAT can be applicable for quantitative data, and for qualitative data when an appropriate distance is defined. The authors of [45] proved that the $k$-Ward's algorithm terminates after a finite number of steps, and that the computational complexity is quadratic (i.e., $\mathrm{O}\left(n^{2}\right)$ ). The following definitions and results are needed to understand the concept of an optimal $k$-partition; the proofs of the respective assertions can be found in [45, 91, 92].

Proposition 1. An optimal solution to the k-partition problem of a data set exists such that each of its groups has a size greater than or equal to $k$ and less than $2 k$ [45].

Definition 1. For a given data set, a $k$-partition $P$ is said to be finer than another $k$-partition $P^{\prime}$ if every group in $P$ is contained by a group in $P^{\prime}[45,91]$.

Definition 2. For a given data set, a $k$-partition $P$ is said to be minimal with respect to the relationship "finer than" if there is no $k$-partition $P^{\prime} \neq P$ such that $P^{\prime}$ is finer than $P[45,91]$.

Proposition 2. For a given data set, a $k$-partition $P$ is minimal with respect to the relationship "finer than" if, and only if, it consists of groups with sizes $\geq k$ and $<2 k$ [45].

Based on the Ward algorithm and the above propositions, the authors of $[45,91]$ have developed the $k$-Ward_MAT algorithm for micro-aggregation. The essential qualifiers for this are the facts that the distance criterion is the $S S E$ and that each group should contain between $k$ and $2 k-1$ elements. The $k$-Ward_MAT algorithm is given below.

The formal proof of the convergence of the $k$-Ward_MAT algorithm can be found in [45]. The experimental results related to $k$-Ward_MAT, found in [45], are included in Section 3.5 of this chapter. 


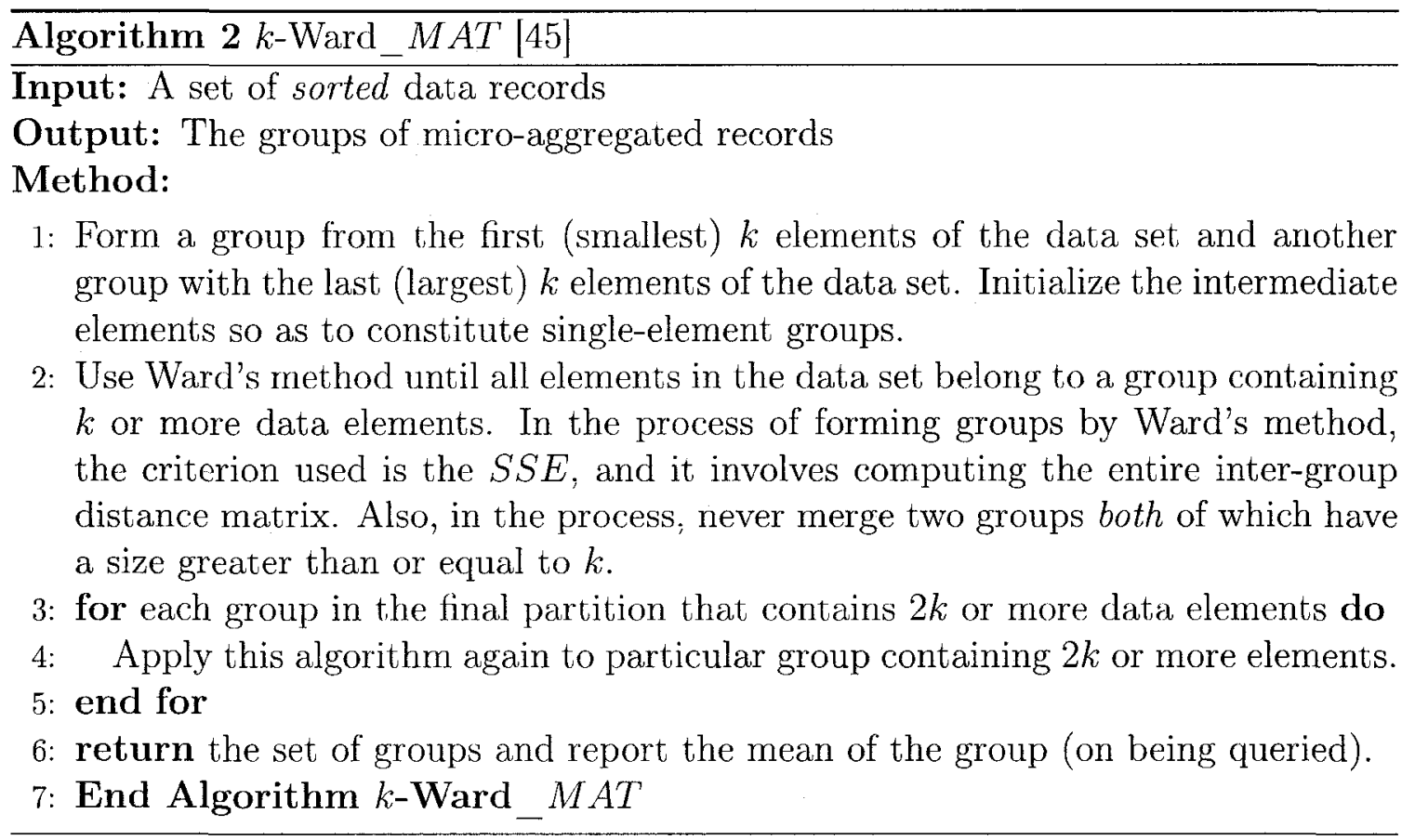

\subsection{Optimized $k$-Ward Micro-aggregation Technique}

The authors of [45] claim that the performance of $M A T$ depends on the distribution of the variables. However, it has been observed that no single method outperforms all other methods for all variables involving real-life data. This conclusion was made after testing all the competing methods for each variable and using the method which yielded the lowest $I L^{5}$. In this chapter, we restrict our study to the $k$-Ward scheme, because it demonstrates a good trade-off between the $I L$ and the $D R$. In addition to this, we also propose an extension of the scheme to multi-variate micro-aggregation after a projection-based preprocessing phase. This has already been achieved, and the results will be included in a later section. In this regard, we observe that there

\footnotetext{
${ }^{5} \mathrm{It}$ is well known that minimizing the two criteria, namely the $I L$ and the $D R$ can have contradictory implications. As researchers, we encounter a real paradox here. Such a study must assess how much information must be hidden to preserve privacy while, at the same time, how much must be retained to make the data usable. Such a discussion is, unfortunately, a study in its own right, and it is not included in this chapter. However, preliminary discussions can be found in $[27,102,124,120]$.
} 
is much room for improvement when it concerns the speed of micro-aggregation. Indeed, the current $k$-Ward's method suffers from two major disadvantages: First, that of the excessive computational burden encountered by processing all the data elements and, additionally, that of computing the "entire" distance matrix that contains the distances between every single pair of groups. Therefore, we propose two modifications by which we can enhance this method and reduce the required time needed to micro-aggregate the data set. These optimizations also lead to enhancements in the multi-variate case.

\subsubsection{Optimizing Distance-Based Computations}

The first modification we achieve is in the Ward method itself [139] and, more specifically, in the phase that computes the distance matrix. The latter is an $m \times m$ matrix, where $m$ represents the number of groups included at each step. It contains the distance values between the groups (recorded as $D_{i j}$ ) that, in turn, represents the $S S E$ obtained by potentially merging $G_{i}$ with $G_{j}$. This matrix is symmetric and has a zero diagonal.

The $k$-Ward's method requires a number of basic steps to generate a near optimal $k$-partition, where the best-case number of steps is $n(1-1 / k)$, and the worst-case number is $(n / k-1)(n / 2+k-2)$ [45]. At each basic step, the number of groups is reduced by unity. Currently, there are two different approaches to compute the value of the distance matrix, namely using either a stored matrix approach, or invoking a stored data approach. In the $k$-Ward method, which uses a stored matrix approach ${ }^{6}$, $\left(m^{2}-m\right) / 2$ values are computed at the initialization step, and $m-1$ values are recomputed at each basic step. But using our newly introduced enhanced version of $k$ Ward_MAT, the so-called $k$-Ward Diagonal, $k W D$, only $m-1$ values are computed in the initial step and at most 2 values are recomputed during a basic step. On the other hand, using a stored data approach ${ }^{7}$ for a $k$-Ward scheme requires no

\footnotetext{
${ }^{6}$ The distance matrix is computed, stored, and retrieved from storage as needed.

${ }^{7}$ The distance matrix is computed when needed rather than retrieved from storage.
} 
initialization step, and it computes $\left(m^{2}-m\right) / 2$ values at each basic step. As opposed to this, $k W D$ computes only $m-1$ values, which lie on the diagonal above the main zero diagonal.

In principle, $k W D$ behaves just like $k$-Ward_MAT, but the primary difference involves the way the Ward algorithm is invoked. While $k$-Ward_MAT invokes the Ward algorithm as in Section 3.2, $k W D$ avoids computing all the values above the main diagonal in order to find the nearest pair of distinct groups. Rather, it computes only the $S S E$ values that lie on the principal diagonal above the main zero diagonal. Consequently, the only change required lies in Step 2 of the Ward algorithm, as shown below.

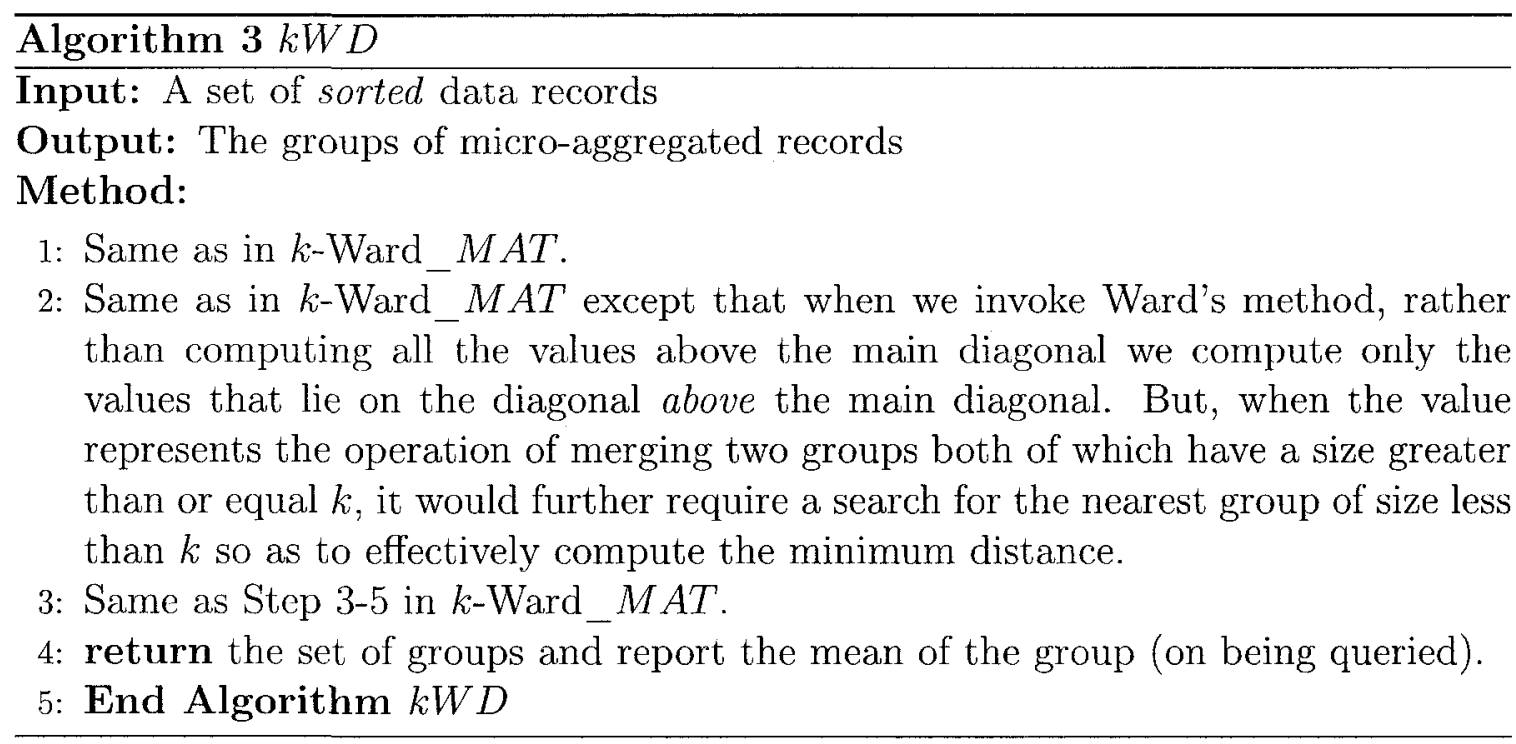

The rationale for optimizing the computation, as in the $k W D$, is based on the mathematical results given presently. Figure 3.1 visualizes the computation of the distance matrix using both the $k$-Ward and the $k W D$ schemes.

Since we are grouping an ordered data set, we present a result that states that the distance value of merging $G_{i}$ with $G_{i+j}$ is less than the distance value of merging $G_{i}$ with $G_{i+k}$, when $k>j$ with a very high probability. This is shown in Theorem 1 , which needs the result of Lemma 1 below. 


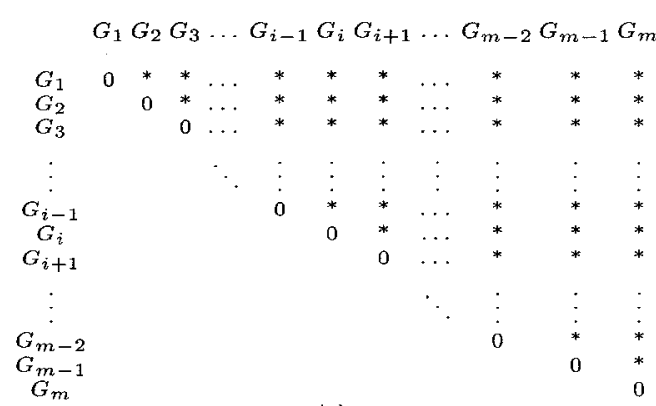

(a)

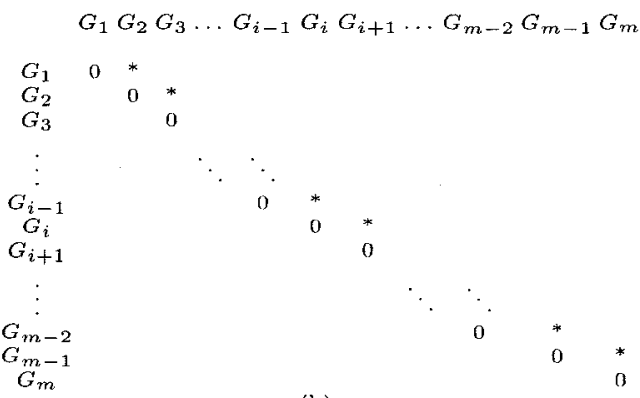

(b)

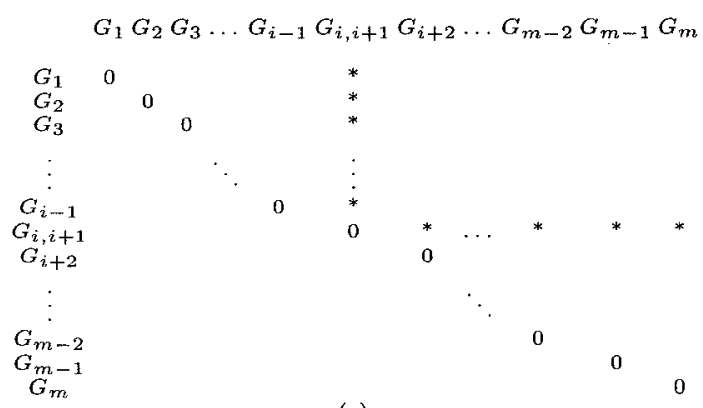

(c)

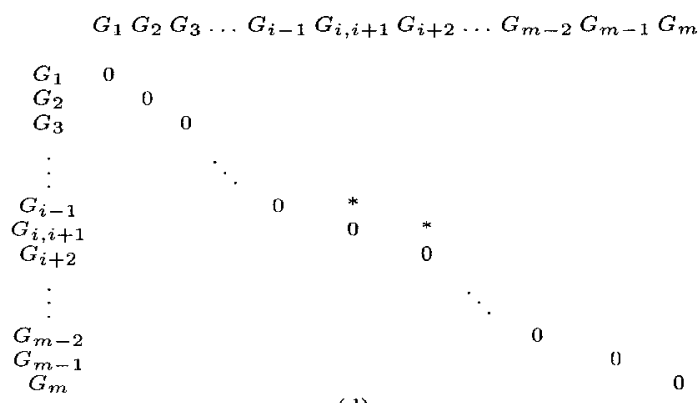

(d)

Figure 3.1: Comparison between $k$-Ward_MAT and the Optimized version $k W D$ in computing the distance matrix using the stored matrix approach. In the initialization step, (a) $k$-Ward_MAT computes all values that lie above the diagonal, while (b) $k W D$ computes only the diagonal which is above the main diagonal. At each basic step, (while merging groups $G_{i}$ with $G_{i+1}$, (c) $k$-Ward $M A T$ computes all values that lie on the column corresponding to $G_{i, i+1}$ and the row $G_{i, i+1}$ above the diagonal, while (d) $k W D$ computes at most two values $\left(G_{i, i+1}, G_{i-1}\right)$ and $\left(G_{i+2}, G_{i, i+1}\right)$.

Lemma 1. Let $\mathbb{P}_{k}^{*}=\left\{G_{1}, G_{2}, G_{3}, \ldots, G_{m}\right\}$ be an optimal partition. Then each partition $G_{i}=\left\{x_{i 1}, x_{i 2}, \ldots, x_{i n_{i}}\right\}$ satisfies this property $x_{i n_{i}} \leq x_{j 1}$, where $i<j$, $1 \leq i<m$ and $1<j \leq m$.

Proof.

Let us assume that $x_{i n_{i}}>x_{j 1}$ in $\mathbb{P}_{k}^{*}$ the optimal partition. Observe that having the optimal partition implies a minimum value of the $I L$, which is directly proportional to the $S S E$. If $x_{i n_{i}}>x_{j 1}$ for any $j>i$, the $S S E$ can be shown to have not attained the minimum value. This is due to the fact that if $\sum_{l=1}^{n_{i}}\left(x_{i l}-\bar{x}_{i}\right)^{2}$ is the 
contribution for $G_{i}$ and $\sum_{l=1}^{n_{j}}\left(x_{j l}-\bar{x}_{j}\right)^{2}$ is the contribution for $G_{j}$, these quantities are not minimized because $x_{j 1}-\bar{x}_{i}<x_{j 1}-\bar{x}_{j}$ as per our assumptions. Consequently, to further minimize the $S S E, x_{j 1}$ should be partitioned into $G_{i}$ and not into $G_{j}$. Thus, the $I L$ obtained if $x_{i n_{i}}>x_{j 1}$ is not the minimum value, which means that $\mathbb{P}_{k}^{*}$ is not an optimal partition. Hence the result.

Theorem 1. Consider the quantity $D\left(G_{i}, G_{j}\right)$, defined as $\frac{n_{i} n_{j}}{n_{i}+n_{j}}\left(\bar{x}_{i}-\bar{x}_{j}\right)^{2}$. Then, if the index $k>j$, and the size of the groups $G_{i+k}$ and $G_{i+j}$ satisfy $n_{i+k} \leq n$ and $n_{i+j} \leq n$ respectively, then

$$
\begin{cases}D\left(G_{i}, G_{i+k}\right) \geq D\left(G_{i}, G_{i+j}\right), & \text { Whenever } n_{i+k} \geq n_{i+j} \\ D\left(G_{i}, G_{i+k}\right) \geq D\left(G_{i}, G_{i+j}\right), & \text { Whenever } n_{i+k}<n_{i+j} \text { and if the groups } \\ & G_{i+j} \text { and } G_{i+k} \text { have different elements } \\ D\left(G_{i}, G_{i+k}\right)<D\left(G_{i}, G_{i+j}\right), & \text { Otherwise. }\end{cases}
$$

Proof.

First of all, observe that by definition, $\forall G_{i}, \bar{x}_{i}=\frac{1}{n_{i}} \sum_{j=1}^{n_{i}} x_{i j}$

Since all the elements in the data set are ranked in an ascending order, it implies that we have the ordering: $\bar{x}_{i} \leq \bar{x}_{i+j} \leq \bar{x}_{i+k}$, where $i=1,2, \ldots$, with $i+j$ and $i+k \leq n$. The distance between groups $G_{i}$ and $G_{j}$ is computed as $D\left(G_{i}, G_{j}\right)=$ $\frac{n_{i} n_{j}}{n_{i}+n_{j}}\left(\bar{x}_{i}-\bar{x}_{j}\right)^{2}[45]$.

The latter is nothing more than the squared difference between the means of the groups weighted by a scalar factor, namely, $\frac{n_{i} n_{j}}{n_{i}+n_{j}}$. For $i=1,2, \ldots$, with $i+j \leq n$ and $i+k \leq n$, we now consider the various cases when (3.1) is true, knowing that (3.2) is always true.

$$
\begin{aligned}
& \left(\bar{x}_{i}-\bar{x}_{i+j}\right)^{2} \leq\left(\bar{x}_{i}-\bar{x}_{i+k}\right)^{2} \\
& \bar{x}_{i} \leq \bar{x}_{i+j} \leq \bar{x}_{i+k}
\end{aligned}
$$


which is equivalent to determining when (3.3) holds:

$$
\frac{n_{i} n_{i+j}}{n_{i}+n_{i+j}}\left(\bar{x}_{i}-\bar{x}_{i+j}\right)^{2} \leq \frac{n_{i} n_{i+k}}{n_{i}+n_{i+k}}\left(\bar{x}_{i}-\bar{x}_{i+k}\right)^{2} .
$$

We consider the three pertinent mutually exclusive and exhaustive cases.

- Case $\left(n_{i+j}=n_{i+k}\right)$

If $n_{i+j}=n_{i+k}$, the multiplying terms $\frac{n_{i} n_{i+j}}{n_{i}+n_{i+j}}=\frac{n_{i} n_{i+k}}{n_{i}+n_{i+k}}$. Since the $\vec{x}_{i}$ s are sorted by virtue of (3.2), a simple algebraic manipulation shows that the $R H S$ of (3.3) is greater than its $L H S$, and (3.3) follows.

- Case $\left(n_{i+j}<n_{i+k}\right)$

The difference between $n_{i+k}$ and $n_{i+j}$ is bounded by $[1, k-1]$ based on Proposition 1 in [45], and so, in the worst case, the difference cannot exceed $k-1$. Thus (3.3) implies:

$$
\begin{aligned}
& \frac{n_{i} n_{i+j}}{n_{i}+n_{i+j}}\left(\bar{x}_{i}-\bar{x}_{i+j}\right)^{2} \leq \frac{n_{i} n_{i+k}}{n_{i}+n_{i+k}}\left(\bar{x}_{i}-\bar{x}_{i+k}\right)^{2} \\
& \Rightarrow \frac{n_{i} n_{i+j}}{n_{i}+n_{i+j}}\left(\bar{x}_{i}-\bar{x}_{i+j}\right)^{2} \leq \frac{n_{i}\left(n_{i+j}+k-1\right)}{n_{i}+\left(n_{i+j}+k-1\right)}\left(\bar{x}_{i}-\bar{x}_{i+k}\right)^{2} \\
& \text { But } \frac{\left(\bar{x}_{i}-\bar{x}_{i+k}\right)^{2}}{\left(\bar{x}_{i}-\bar{x}_{i+j}\right)^{2}} \geq 1 \text {, and } \\
& \frac{\left(n_{i}+n_{i+j}+k-1\right) n_{i+j}}{\left(n_{i}+n_{i+j}+k-1\right) n_{i+j}+(k-1) n_{i}}<1 \quad \quad \forall k>1 \\
& \text { Thus } \frac{\left(n_{i}+n_{i+j}+k-1\right) n_{i+j}}{\left(n_{i}+n_{i+j}+k-1\right) n_{i+j}+(k-1) n_{i}}<\frac{\left(\bar{x}_{i}-\bar{x}_{i+k}\right)^{2}}{\left(\bar{x}_{i}-\bar{x}_{i+j}\right)^{2}} \text {, and so (3.3) follows. }
\end{aligned}
$$

- Case $\left(n_{i+j}>n_{i+k}\right)$

As in the above case, we know that $\frac{\left(\bar{x}_{i}-\bar{x}_{i+k}\right)^{2}}{\left(\bar{x}_{i}-\bar{x}_{i+j}\right)^{2}} \geq 1$. Again, the maximum difference between $n_{i+j}$ and $n_{i+k}$ is $k-1$. Since we are grouping data which is ordered, we have:

$$
\frac{\left(n_{i}+n_{i+j}+k-1\right) n_{i+j}+(k-1) n_{i}}{\left(n_{i}+n_{i+j}+k-1\right) n_{i+j}}>1
$$

The value of $\frac{\left(\bar{x}_{i}-\bar{x}_{i+k}\right)^{2}}{\left(\bar{x}_{i}-\bar{x}_{i+j}\right)^{2}}$ is almost always greater than $\frac{\left(n_{i}+n_{i+j}+k-1\right) n_{i+j}+(k-1) n_{i}}{\left(n_{i}+n_{i+j}+k-1\right) n_{i+j}}$ except in the single case when $\bar{x}_{i+j}=\bar{x}_{i+k}$ as shown in Lemma 1. This case occurs only when both $G_{i+j}$ and $G_{i+k}$ have elements with a single repetitive entry (with different frequencies). More precisely $G_{i+j}$ and $G_{i+k}$ contain the same quantity, $v$, with group sizes equal to $n_{i+j}$ and $n_{i+k}$, respectively. Thus $\bar{x}_{i+j}=\bar{x}_{i+k}$, and the magnitude of the quantity $\frac{\left(n_{i}+n_{i+j}+k-1\right) n_{i+j}+(k-1) n_{i}}{\left(n_{i}+n_{i+j}+k-1\right) n_{i+j}}$ 


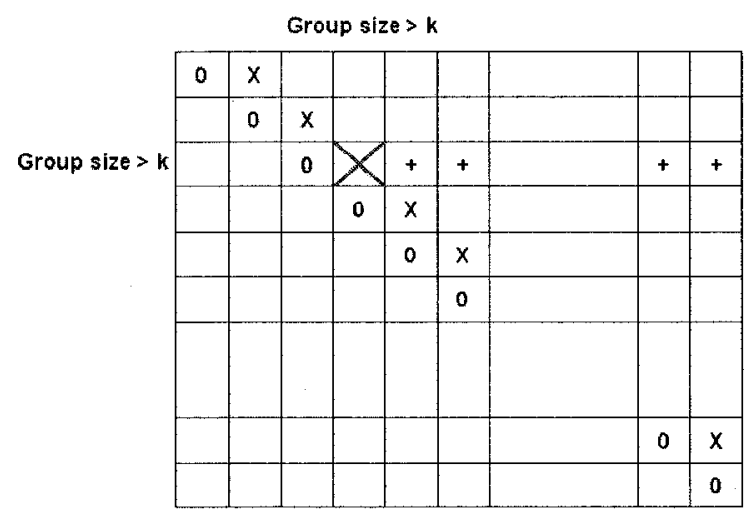

Figure 3.2: Components of the similarity distance matrix for a micro-data file that have to be computed for algorithm $k W D$

will play the predominant role in determining the value of the minimum distance in the distance matrix. In this case $D\left(G_{i}, G_{i+k}\right) \leq D\left(G_{i}, G_{i+j}\right)$, which specifies the only case where (3.3) does not follow.

The theorem follows.

Theorem 1 can be used to reduce the computational time required for determining the nearest pair of distinct groups to be merged in Step 2 in the $k$-Ward_MAT algorithm. As shown above, the condition $D\left(G_{i}, G_{i+k}\right) \geq D\left(G_{i}, G_{i+j}\right)$ is almost always true, except in a single case where both groups $G_{i+j}$ and $G_{i+k}$ have elements with a single repetitive entry - which is an event occurring with a very small probability. Thus, if this event is ignored, it is obvious that computing the distances between $G_{i}$ and $G_{i+1}$ for all $i \leq n-1$ is sufficient to determine the minimum distance value in the distance matrix. It turns out that, due to the ordering of the elements (and the sets), the values of these distances lie on the principal diagonal above the main (zero) diagonal. This is, indeed, what the $k W D$ algorithm achieves at Step 2, thus significantly reducing the computational time.

Theorem 2. Algorithm KWD produces the same output as the $k$-Ward strategy. 
Proof.

Before proceed, we observe that by virtue of the previous result, algorithm $k W D$ will be considered correct if it correctly achieves the following steps:

1. Computing the values, which lie on the diagonal above the main zero diagonal. As argued above, this is adequate when it concerns determining the minimum distance between groups in the similarity distance matrix.

2. Ensuring that none of the values which lie on the diagonal above the main zero diagonal, represent the merging of two groups both of which have a size greater than or equal to $k$.

In the case when both the merged groups have a cardinality less than $k$, the first point above follows directly as a consequence of Lemma 1 and Theorem 1. This is because the values which lie on the diagonal above the principal diagonal, truly evaluate the minimum distance values among the entire sct of values above the main zero diagonal. More formally, this is true as a consequence of the fact that $D\left(G_{i}, G_{i+k}\right) \geq D\left(G_{i}, G_{i+j}\right)$ for $i+j \leq m, i+k \leq m$, and $k>j$.

The argument becomes marginally more complex when the groups involved have cardinalities greater than $k$, which represents the second condition. In this case, if the distance value represents the event associated with such a merge, the $k$-Ward_MAT will search for the next (or sccond) minimum distance between two groups along the same row, and will then merge them. The next minimum distance value is the nearest (nonadjacent) neighbor on the same row, whose cardinality is less than $k$. Observe that this is analogous to Step 2 of algorithm $k W D$, where for every group (say, with row-index $i$ ). which has a cardinality greater than or cqual to $k$, the latter algorithm seeks out a group (with column index, $j$ ) whose cardinality is less than $k$. Thus, to determine the group with the associated column, all we need to do is to traverse the row from index $i+1$ till we find such a group. This is, of-course, true by virtue of the non-increasing sequence of distances along the row, (namely, those indicated by the ' + ' symbol in the associated row as in Figure 3.2). During the traversal, we either 
encounter a group of size less than $k$, or result in the conclusion that no such group exists. In the first scenario, the computed distance value will be the entry used for the minimum distance candidates. As opposed to this, in the second scenario, the minimum value will be one of the remaining values which lie on the diagonal above the zero diagonal (see Figure 3.2). Since these distances are computed in the body of the loop, the theorem follows.

\subsubsection{Recursive $k$-Ward Optimization}

Having discussed how the $k$-Ward computation can be improved for a single partitioning, we now propose a recursive and superior mechanism, referred to as Recursive $k$-Ward Micro-aggregation, $k W R$, for further minimizing the computations for the entire data set. Our strategy is the following: Rather than process all the data using a $k$-Ward method, we propose that the data be recursively subdivided into smaller subsets. We emphasize that the smaller subsets need not be obtained as the result of invoking a $k$-Ward algorithm on the original data. This strategy leads to a sequence of so-called divide- $k$-Ward-coalesce steps, which are invoked recursively to ultimately yield the desired micro-aggregated records. This recursive subdivision can be "arbitrary" (i.e., based on any meaningful criterion), and it does not need to utilize any underlying clustering methodology. Furthermore, we propose that each subset be micro-aggregated independently. Finally, the micro-aggregated records are combined in order to obtain the entire set of records appropriately grouped.

The algorithm that implements the $k W R$ can be formalized as follows. Let the input data set be given by InSet and the output micro-aggregated records be OutSet. We first partition the InSet set into $J$ mutually exclusive subsets InSet $_{1}$, InSet $_{2}, \ldots$, InSet $_{J}$. We then compute the SST for this set and for each subset. Subsequently, we calculate the value of $\frac{\Sigma_{i=1}^{J} S S T\left(\text { InSet } t_{i}\right)}{S S T(I n S e t)}$ and compare it to a user-defined threshold, $\theta$. If the value of $\frac{\sum_{i=1}^{J} S S T\left(\text { InSet }_{i}\right)}{S S T(\text { InSet })}$ is smaller than $\theta$, a traditional $k$-Ward MAT is invoked to yield the micro-aggregated records. Otherwise, the original data set is further recursively subdivided into the $J$ sub-subsets, and the process 
is recursively invoked, thus proceeding towards the leaves of the recursive tree. Observe that a traditional $k$-Ward $M A T$ is invoked only when the corresponding input set is not only small enough but when it also preserves the minimization of the $I L$.

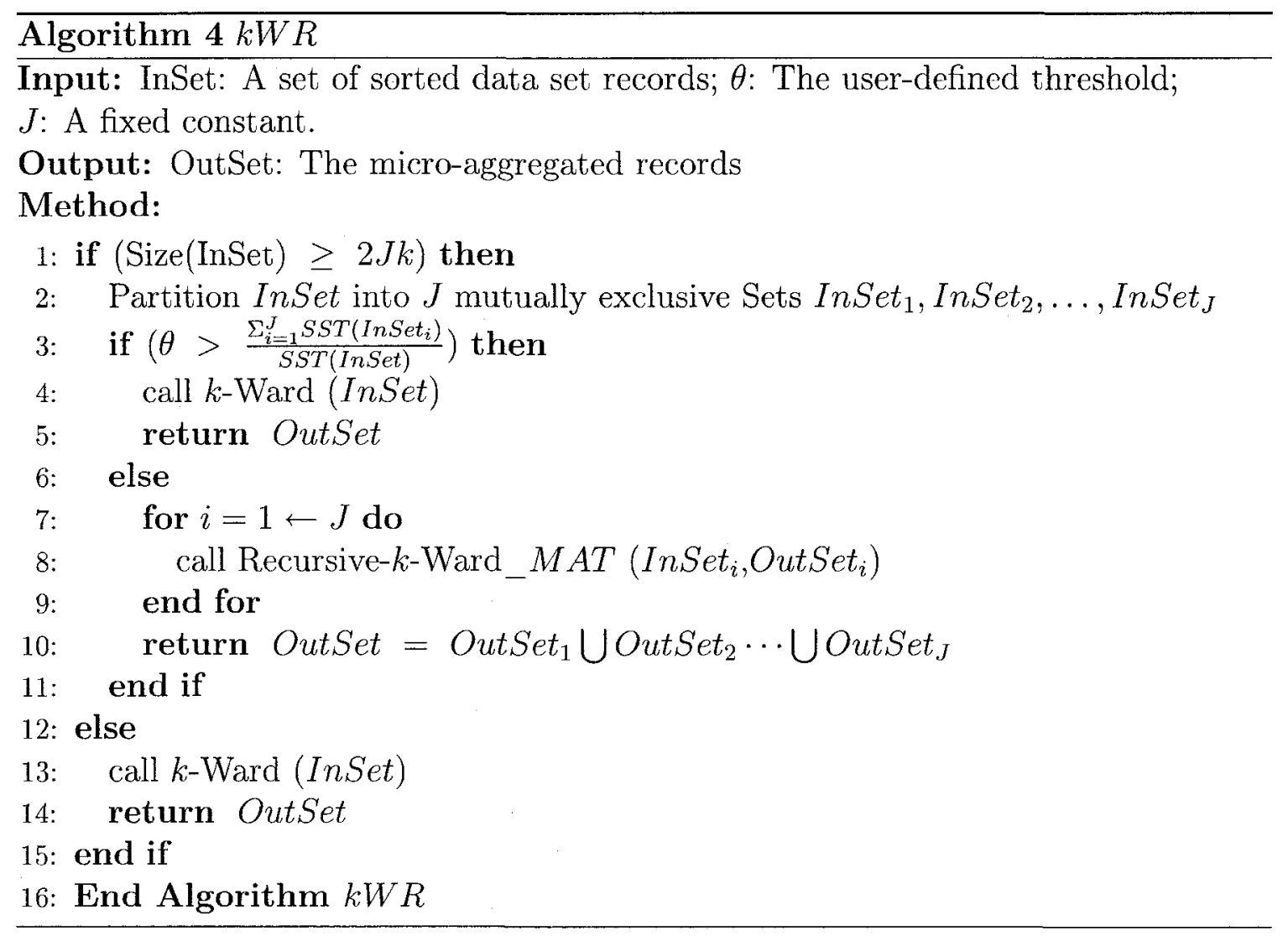

The proposed recursive method is consistent with our overall objectives of minimizing the $I L$, minimizing the $D R$, and reducing the required time to micro-aggregate all the records in the data set. The main advantage of this modification, compared to the corresponding non-recursive version, is that $k W R$ can micro-aggregate the data set in significantly less time without sacrificing either the $I L$ or the $D R$. Another advantage of such a strategy is that such a modification will provide a natural way to design an efficient algorithm inherently suitable for multi-processor machines, and especially for shared-memory systems where the communication of data between processors does not have to be planned in advance because distinct subsets can be 
executed on different processors. Apart from this, such a method can also make efficient use of memory caches because after a subset is small enough to be stored in cache the partitioning can be achieved without accessing the slower main memory.

The most intricate part of designing such a recursive method involves determining a suitable threshold value, $\theta$, because it depends on the structure, distribution, and statistical measures of the data set. Indeed, from our experience, a good initial guess of $\theta$ is generally around the value of $\frac{\sum_{i=1}^{J} S S T\left(I n S e t_{i}\right)}{S S T(I n S e t)}$.

Using a recursive strategy ensures that at least $J$ subsets are obtained from the entire data set, which leads to a noticeable saving on time. Further, by this recursive method we not only reduce the required time to micro-aggregate the records, but also attempt to preserve the minimization of the $I L$. This is achieved by invoking the base (terminating) step, where the $I L$ is minimized for each atomic partition.

Theorem 3. The running time for the $k W R$ Algorithm is bounded by $\left[\bigcirc(n \log n), \bigcirc\left(n^{2}\right)\right]$.

Proof.

The proof of our complexity claim contains two parts:

1. We first prove that the upper bound of the complexity of algorithm $k W R$ is given by $\bigcirc\left(n^{2}\right)$.

To do this, we observe that the worst case of algorithm $k W R$ will occur when the data elements are maximally close to each other. This occurs when there are no gaps between the various data elements, leading to a satisfaction of the $\theta$ condition on Line 3 . Such a scenario will lead to the further invocation of the original $k$-Ward algorithm, which, in the worst case, leads to an obvious complexity of order $\bigcirc\left(n^{2}\right)$ in.

2. We now prove that the lower bound of the time complexity of algorithm $k W R$ is given by $\bigcirc(n \log n)$.

The best case of algorithm $k W R$ occurs when the data is full of gaps that renders the $\theta$ condition (see Line 3 ) to be always False. The size of the Inset 
data set will then determine the complexity of the algorithm. We see that there are three mutually exclusive and exhaustive cases:

- Case 1. This is the case when the size of $\operatorname{InSet,~} n$, is greater than or equal $2 J k$. Such a case will cause the recursive invocation of algorithm $k W R J$ times after the $\theta$ condition fails. This will require $O(n)$ time to compute the SST for each subset.

- Case 2. This is the case when the size of InSet, $n$, is less than $2 J k$ and greater than $2 k$. In this case, the latter cannot be further divided into $J$ subsets. Hence, the original $k$-Ward algorithm will be invoked, leading to a time complexity of $\bigcirc\left(n^{2}\right)$.

- Case 3. When the size of InSet is equal to $2 k$, this will further invoke the $k$-Ward algorithm. Such an invocation will place the first $k$ elements in the first, subset, and the last $k$ elements in the second group. Thus, since there are no elements between the two subsets, the algorithm terminates with $\bigcirc(1)$ computations $^{8}$. Now, it is clear that the event that InSet has a size smaller than $2 k$ is an event of probability zero, because when the size of $I n S e t$ is less than $2 J k$, it will not be further divided into $J$ subsets. This implies that this event cannot occur.

Merging these arguments, we see that the running time of algorithm $k W R$ is $T(n)$, where:

$$
T(n)= \begin{cases}J T(n / J)+\bigcirc(n), & n \geq 2 J k \\ \bigcirc\left(n^{2}\right), & 2 k<n<2 J k \\ \bigcirc(1), & n \leq 2 k\end{cases}
$$

By considering the different "paths" of the event tree when the above scenarios can occur, it can be shown that ${ }^{9}$ :

$$
T(n)=\sum_{i=0}^{\log _{j}(n-2 J k)} J * \frac{n}{J}+\sum_{i=2 k}^{2 J k} \bigcirc\left(i^{2}\right)+\bigcirc(1)
$$

\footnotetext{
${ }^{8}$ This is, quite simply, because the set is already sorted.

${ }^{9}$ The details are omitted in the interest of brevity.
} 
Consider the first term. By using the so-called Mastering method [18], we can see that $\sum_{i=0}^{\log _{j}(n-2 J k)} J * \frac{n}{J}=\bigcirc(n \log n)$. Similarly, when considering the second term, we see that $\sum_{i=2 k}^{2 J k} \bigcirc\left(i^{2}\right)=\bigcirc(1)$ because $k$ and $J$ are constants, and the values of both of these terms are negligible comparable to $n$, the size of the data. Thus, the best case running time of algorithm $k W R$ is $\bigcirc(n \log n)$.

The theorem follows.

\subsection{Experimental Results}

In this section, we describe our data sets, explain our experimental methods, and present the results we have obtained by using $k W D, k W R$, and $k W D R$ for microaggregation. All the programs were written in the $C^{++}$language, and the tests were performed on an Intel(R) Pentium (R)M Processor with the clock speed of $1.73 \mathrm{GHz}$, and with $512 M B$ of $R A M$.

The data used in the experiments is given in Section 3.5.1. Sections 3.5.2 and 3.5 .3 report the results for the univariate $M A T$. The corresponding results for the projected multi-variate data sets are given in Section 3.5.4.

\subsubsection{Data Sets}

We tested the various versions of the $k$-Ward_MAT using the two reference data sets $^{10}$ that have been used as benchmarks in previous studies [45, 53]:

\section{Tarragona Data Set [45]}

This set involves 834 companies in the Tarragona area. Each company has 13 associated quantitative variables, as follows: Fixed_Assets (Var1), Current_Assets (Var2), Treasury (Var3), Uncommitted_Funds (Var4), Paid-up_Capital

\footnotetext{
${ }^{10}$ In future, we shall merely refer to these data sets as the Tarragona and Census data sets.
} 
(Var5), Short-term _Debt (Var6), Sales (Var7), Labor_Cost (Var8), Description (Var9), Operating_Profit (Var10), Financial_Outcome (Var11), Gross_Profit (Var12), and Net_Profit (Var13). Table 3.1 shows the main descriptive statistics of the Tarragona data set.

Table 3.1: Statistical Summary for Tarragona data set

\begin{tabular}{|c|c|c|c|c|c|c|}
\hline \hline \multicolumn{7}{|c|}{ Tarragona Data Set } \\
\hline Variable & Maximum & Minimum & Mean & Median & Mode & Std.Dev. \\
\hline Var1 & 4994098 & 0 & 105338.8261 & 33930.5 & 0 & 278227.8001 \\
\hline Var2 & 4539074 & -26538 & 210314.0204 & 90545 & 0 & 432394.3559 \\
\hline Var3 & 497490 & -38776 & 19352.86811 & 5656 & 0 & 44704.9887 \\
\hline Var4 & 3939325 & -515464 & 120218.6571 & 40580 & 769 & 303023.5211 \\
\hline Var5 & 1493787 & 90 & 39946.43165 & 11000 & 10000 & 105988.4465 \\
\hline Var6 & 3601182 & -2701 & 159344.012 & 67045 & 10393 & 326846.4954 \\
\hline Var7 & 15382214 & 0 & 546958.2818 & 244794.5 & 277939 & 1155792.694 \\
\hline Var8 & 1372449 & 0 & 74447.95923 & 36200 & 0 & 135407.5982 \\
\hline Var9 & 378927 & -119 & 10855.10312 & 3614 & 0 & 27193.58137 \\
\hline Var10 & 1207056 & -251798 & 27622.76499 & 8475.5 & 4750 & 88221.54817 \\
\hline Var11 & 108058 & -357071 & -8366.248201 & -3548 & 0 & 25690.3612 \\
\hline Var12 & 1267541 & -301172 & 21243.48441 & 4055 & 2580 & 80287.94397 \\
\hline Var13 & 873843 & -301172 & 14133.80096 & 2844.5 & 1955 & 56155.53656 \\
\hline \hline
\end{tabular}

\section{Census Data Set [53]}

This set was obtained on July 27, 2000, using the Data Extraction System of the U.S. Bureau of the Census. It contains 1,080 records that were obtained using the extraction procedure described in [14], and it has 13 quantitative variables ${ }^{11}$ : Final_Weight (Var1), Adjusted_Income (Var2), Insurance_Contribution (Var3), Business_Earnings (Var4), Federal_Tax (Var5). Payroll_Deduction (Var6), Interest_Income (Var7), Total_Earnings (Var8), Total_OIncome (Var9), Total_Income (Var10), State_Tax (Var11), Taxable Income (Var12), and Amount (i.e., total wage and salary) (Var13). Table 3.2 shows the main descriptive statistics of the Census data set.

\footnotetext{
${ }^{11}$ The full forms of the variables can be found in [14].
} 
Table 3.2: Statistical Summary for Census data set

\begin{tabular}{|c|c|c|c|c|c|c|}
\hline \hline \multicolumn{7}{|c|}{ Census Data Set } \\
\hline Variable & Maximum & Minimum & Mean & Median & Mode & Std.Dev. \\
\hline Var1 & 689039 & 13567 & 196039.81 & 180349.00 & 13567 & 101251.42 \\
\hline Var2 & 99894 & 6539 & 56222.76 & 58412.50 & 6539 & 24674.84 \\
\hline Var3 & 7091 & 16 & 3173.14 & 3215.50 & 16 & 1401.83 \\
\hline Var4 & 21260 & 1 & 7544.66 & 7068.00 & 1 & 4905.20 \\
\hline Var5 & 116721 & 3570 & 45230.84 & 43278.00 & 3570 & 21323.47 \\
\hline Var6 & 11480 & 2 & 2597.18 & 2322.00 & 2 & 1826.44 \\
\hline Var7 & 83454 & 8 & 39712.95 & 41155.00 & 8 & 21224.16 \\
\hline Var8 & 105941 & 1 & 5162.23 & 1586.50 & 5000.00 & 9449.64 \\
\hline Var9 & 49425 & 1 & 1421.41 & 353.00 & 500.00 & 3750.89 \\
\hline Var10 & 97604 & 80 & 40068.61 & 39000.00 & 30000.00 & 20816.01 \\
\hline Var11 & 7932 & 6 & 2962.65 & 3002.00 & 4207.00 & 1427.23 \\
\hline Var12 & 97604 & 80 & 39523.38 & 38000.00 & 30000.00 & 20601.28 \\
\hline Var13 & 97604 & 80 & 38444.56 & 36000.00 & 30000.00 & 20677.57 \\
\hline \hline
\end{tabular}

\subsubsection{Results for Uni-variate MATs}

The run time characteristics of the optimized $k$-Ward micro-aggregation algorithms, (for the uni-variate case), for the data sets are discussed in more detail in this section.

The sorted data approach was chosen to obtain the specific implementation of $k W D$. Table 3.3 presents the results obtained by executing $k W D$ and the $I L 100$ times for each variable. From the table we see that $k W D$ leads to a huge reduction in the running time required to micro-aggregate all the records in the data set compared to the original $k$-Ward_MAT. For example, the required time to micro-aggregate the values of Var11 in the Tarragona data set using $k$-Ward_MAT was 19.86 seconds while it was only 3.95 seconds using $k W D$. The percentage of time improvement reaches up to $80.11 \%$ on the Tarragona data set. Similarly, the required time to micro-aggregate all values of $\operatorname{Var} 2$ in the Census data set was 42.41 seconds using $k$-Ward $M A T$, but the $k W D$ required only 8.36 seconds. Again, the percentage of time improvement is as high as $80.29 \%$ on the Census data set. Interestingly, both the $k$-Ward_MAT and $k W D$ obtain the same value of the $I L$ for each variable in both the data sets. 
Table 3.3: Comparison between the original $k$-Ward_MAT, $k W$, and the optimized $k W D$ on the Tarragona and the Census data sets. In this case we used the value of $k=3$.

\begin{tabular}{|c|c|c|c|c|c|c|c|c|c|c|c|}
\hline \multicolumn{6}{|c|}{ Tarragona Data Set } & \multicolumn{6}{|c|}{ Census Data Set } \\
\hline \multirow[t]{2}{*}{ Var } & \multicolumn{2}{|c|}{$k W$} & \multicolumn{3}{|c|}{$k W D$} & \multirow[t]{2}{*}{ Var } & \multicolumn{2}{|c|}{$k W$} & \multicolumn{3}{|c|}{$k W D$} \\
\hline & IL & Time & $\overline{\mathrm{IL}}$ & Time & Improv. $(\%)$ & & $\mathrm{IL}$ & Time & IL & Time & Improv. (\%) \\
\hline Var1 & 7.176 & 19.89 & 176 & 5.41 & $72.80 \%$ & Var1 & 0.131 & 42.44 & 0.131 & 9.48 & $77.66 \%$ \\
\hline Var2 & 0.557 & 19.72 & 0.557 & 4.98 & $74.75 \%$ & Var2 & 0.001 & 42.41 & 0.001 & 8.36 & $80.29 \%$ \\
\hline Var3 & 0.775 & 19.77 & $\overline{.775}$ & 4.99 & $74.76 \%$ & $\operatorname{Var} 3$ & 0.008 & 42.58 & 0.008 & 8.42 & $80.23 \%$ \\
\hline Var4 & .487 & 19.61 & .487 & 4.88 & $75.11 \%$ & Var4 & 0.005 & 42.03 & 0.005 & 8.84 & $7 \%$ \\
\hline Var5 & 2.018 & 49.03 & 2.018 & 12.78 & 73. & Var5 & 0.024 & $\mid 41.92$ & 0.024 & 8.91 & $5 \%$ \\
\hline Var6 & 0.586 & 19.69 & .586 & 5.03 & 74. & Var6 & 0.034 & 42.00 & 0.034 & $\overline{9.14}$ & $78.24 \%$ \\
\hline Var7 & 1.921 & 19.81 & .921 & 5.03 & 74. & Var7 & 0.002 & 42.06 & 0.002 & 8.36 & $80.12 \%$ \\
\hline Var8 & 0.338 & 19.73 & .338 & 4.95 & 74. & Var8 & 0.443 & 41.69 & 0.443 & 10.66 & $3 \%$ \\
\hline Var9 & 1.287 & 20.02 & .287 & 5.47 & 72. & Var9 & 0.732 & 44.84 & 0.732 & 10.05 & $77.59 \%$ \\
\hline $\operatorname{ar10}$ & 1.905 & 19.6 & .905 & 4.67 & 76 . & $\operatorname{Var10}$ & 0.003 & 47.0 & 0.003 & 10.34 & $1 \%$ \\
\hline ar11 & 2.966 & 19.86 & 2.966 & 3.95 & 80 . & $\operatorname{Var} 11$ & 0.008 & 47.1 & 0.008 & 10.28 & $78.18 \%$ \\
\hline Var12 & 4.748 & 19.72 & 4.748 & 4.72 & 76. & Var12 & 0.004 & 47.31 & 0.004 & $\mid 10.28$ & $78.27 \%$ \\
\hline $\operatorname{ar13}$ & 4.958 & 19.84 & 4.958 & \begin{tabular}{|l|}
4.83 \\
\end{tabular} & $75.66 \%$ & Var13 & 0.005 & $\mid 48.34$ & 0.005 & 10.59 & $78.09 \%$ \\
\hline
\end{tabular}

In the case of $k W R$, the data set was partitioned into 2 subsets $(J=2)$ whenever the base condition was not satisfied. As explained previously, finding the best threshold value, $\theta$, is far from trivial. Indeed, the value of $\theta$ plays an important role in determining the required time, and also in determining the value of the $I L$. Thus, if $\theta$ has a certain value, (which does not allow the base condition to be satisfied), it is clear that the recursion will be invoked more often; this will lead to a huge reduction in the running time, but to an increased value in the $I L$. It is important to mention that the number of recursions invoked differs from one variable to another, but for each specific variable the number of recursive calls seems to be the same regardless of the value of $k$. This makes sense because the recursion divides the data set into subsets, as per the base condition, before invoking the micro-aggregation method. Besides, we also observe that the base terminating condition is generally satisfied at the same positions for each specific variable regardless of the value of $k$. Finally, we have observed that changing the value of $k$ affects the value of the $I L$ and the running time, but it does not seem to affect the total number of recursive invocations. 
Tables 3.4 and 3.5 show a comparison between the original $k$-Ward_MAT and $k W R$ on the Tarragona and Census data sets, respectively. In the table, the sequence of recursive calls is represented by an expression of well-matched parentheses. Thus, for example, there are two recursive calls for the variable Var2 in the Tarragona data set, and this is represented by the parenthetic string " $(()(()()))$ ". The first time the algorithm is invoked, it divided the entire data set into two partitions, each recursively invoking the algorithm again. The first partition was continued with no further divisions or recursions, while the second partition was divided into another two sub-partitions and the algorithm was recursively invoked for each sub-partitions, which ultimately terminated the process. It turns out that in this case the value of $\theta=0.8$ is suitable for all the 13 variables of the Tarragona data set, and the percentage of the time improvement reaches up to $93.65 \%$ on var 12 after 8 recursive calls. But for the Census data set, we observe that we have to set $\theta$ differently for each variable, and that values of $\theta$ - which are respectively $0.4,0.28,0.31,0.28,0.31,0.38$, $0.29,0.70,0.80,0.31,0.27,0.32$, and 0.30 (respective to the corresponding variables $\operatorname{Var} 1$ to $\operatorname{Var} 13)$ - lead to the best solution. $k W R$ yielded the same value of the $I L$ in most of these variables, except for the cases of Var4, Var $5, \operatorname{Var} 6, \operatorname{Var} 11$, and $\operatorname{Var} 12$ where it gave a value close to the value of $I L$ obtained by the original $k$-Ward_MAT as shown in Table 3.5. We believe that the reason for this minor deviation from the optimal is due to the nature of the data. An examination of the values of data in the Tarragona data set shows that since they are relatively close to each other, there are not many gaps between the values. But in the case of the Census data set such gaps exist. Thus, there are many divisions on the Census data set, which will marginally increase the $I L$. However, we still believe that such a small loss is worth the significant computation gain.

\subsubsection{Dynamic threshold}

Since we are attempting to reduce the computational time and the $I L$, we have also experimented the schemes using a dynamic threshold, which varies at each step. This 
Table 3.4: Comparison between the original $k$-Ward_MAT, $k W$, and the optimized $k W R$ using a fixed threshold on the Tarragona data set. In this case we used the value of $k=3$.

\begin{tabular}{|c|c|c|c|c|c|c|}
\hline \multirow[t]{2}{*}{ Var } & \multicolumn{2}{|c|}{$k \mathrm{~W}$} & \multicolumn{4}{|r|}{$k W R$} \\
\hline & $\overline{\mathrm{IL}}$ & Time & IL & Time & Improv. $(\%)$ & Recursion \\
\hline Var1 & 7.176 & 19.89 & 7.176 & 5.31 & $\overline{73.30 \%}$ & $\overline{((()(()(()())))}$ \\
\hline Var2 & 0.557 & 19.72 & 0.557 & 3.75 & $80.98 \%$ & $(0(0)(0))$ \\
\hline Var3 & 0.775 & 19.77 & 0.775 & 5.91 & $70.11 \%$ & $(0)$ \\
\hline Var4 & 1.487 & 19.61 & 1.487 & 1.49 & $92.40 \%$ & $(((((()))())())())(()()))$ \\
\hline Var5 & 2.018 & 49.03 & 2.018 & 22.36 & $54.40 \%$ & $(0(0))$ \\
\hline Var6 & 0.586 & 19.69 & 0.586 & 3.70 & $81.21 \%$ & $(0(0)(0))$ \\
\hline $\operatorname{Var} 7$ & 1.921 & $\mid 19.81$ & 1.922 & 3.75 & $81.07 \%$ & $(()(()()))$ \\
\hline Var8 & 0.338 & 19.73 & 0.338 & 3.75 & $80.99 \%$ & $(0(00))$ \\
\hline Var9 & 1.287 & 20.02 & 1.287 & 3.83 & $80.87 \%$ & $(0(00))$ \\
\hline Var10 & 1.905 & 19.69 & 1.909 & 1.23 & $93.75 \%$ & $((((0) 0)(0)(0)(0)(00)))$ \\
\hline$\overline{\text { Var11 }}$ & 2.966 & 19.86 & 2.967 & 1.99 & $89.98 \%$ & $((0)())(()()))$ \\
\hline Var12 & 4.748 & 19.72 & 4.748 & 1.19 & $93.97 \%$ & $\overline{((((((()())())())())())(()(()())))}$ \\
\hline Var13 & 4.958 & 19.84 & 4.958 & 1.26 & $93.65 \%$ & $((((((()))(0)())())(0)(0)(0))$ \\
\hline
\end{tabular}

Table 3.5: Comparison between the original $k$-Ward_MAT, $k W$, and the optimized $k W R$ using a fixed threshold on the Census data set. In this case we used the value of $k=3$.

\begin{tabular}{|c|c|c|c|c|c|c|}
\hline \multirow{2}{*}{ Var } & \multicolumn{2}{|c|}{$k$ W } & \multicolumn{3}{|c|}{$k W R$} \\
\cline { 2 - 5 } & IL & Time & IL & Time & Improv.(\%) & Recursion \\
\hline \hline Var1 & 0.13 & 42.44 & 0.13 & 12.73 & $70.00 \%$ & $(()(()()))$ \\
\hline Var2 & 0.00 & 42.41 & 0.00 & 7.78 & $81.66 \%$ & $(()(()()))$ \\
\hline Var3 & 0.01 & 42.58 & 0.01 & 6.94 & $83.70 \%$ & $(()(()(()(()(()())))))$ \\
\hline Var4 & 0.00 & 42.03 & 0.01 & 7.02 & $83.30 \%$ & $(()(()(()(()(()((()())(()(()(()(()()))))))))))$ \\
\hline Var5 & 0.02 & 41.92 & 0.03 & 7.13 & $82.99 \%$ & $(()(()(()(()(()(()(()((()())(()(()()))))))))))$ \\
\hline Var6 & 0.03 & 42.00 & 0.08 & 6.97 & $83.40 \%$ & $(()(()(()(()((()())(()(()())))))))$ \\
\hline Var7 & 0.00 & 42.06 & 0.00 & 12.45 & $70.40 \%$ & $(()())$ \\
\hline Var8 & 0.44 & 41.69 & 0.44 & 12.36 & $70.35 \%$ & $(()())$ \\
\hline Var9 & 0.73 & 44.84 & 0.73 & 9.95 & $77.81 \%$ & $(()(()()))$ \\
\hline Var10 & 0.00 & 47.02 & 0.01 & 13.59 & $71.10 \%$ & $(()(()(()((()())()))))$ \\
\hline Var11 & 0.01 & 47.11 & 0.11 & 5.23 & $88.90 \%$ & $((((()())((()(()(()())))(()(()((()(()()))(\ldots)()))))))))$ \\
\hline Var12 & 0.00 & 47.31 & 0.03 & 13.67 & $71.11 \%$ & $(()(()(()(()()))))$ \\
\hline Var13 & 0.00 & 48.34 & 0.01 & 16.44 & $65.99 \%$ & $(()(()(()(()()))))$ \\
\hline
\end{tabular}


dynamic threshold was computed as:

$$
\theta=\frac{S S T\left(\text { InSet }_{1}\right)+S S T\left(\text { InSet }_{2}\right)}{S S T(\text { InSet })} .
$$

At each step, the dynamic threshold was passed as a parameter to the invoked recursion. Tables 3.6 and 3.7 show the power of the dynamic threshold scheme that yields the same value of the $I L$ obtained by original $k$-Ward_MAT on both the Tarragona and Census data sets. Additionally the computation time is reduced significantly and, thus, in one example, the percentage of the time improvement reaches up to $91.04 \%$ on $\operatorname{Var} 11$ in the Tarragona data set, while it is $45.44 \%$ on $\operatorname{Var} 3$ in the Census data set.

Table 3.6: Comparison between the original $k$-Ward_MAT, $k W$, and the optimized $k W R$ using a dynamic threshold on the Tarragona data set. In this case we used the value of $k=3$.

\begin{tabular}{|c|c|c|c|c|c|c|}
\hline \multirow{2}{*}{ Var } & \multicolumn{2}{|c|}{$k W$} & \multicolumn{4}{|c|}{$k W R$} \\
\cline { 2 - 6 } & IL & Time & IL & Time & Improv.(\%) & Recursion \\
\hline \hline Var1 & 7.18 & 19.89 & 7.18 & 10.30 & $48.22 \%$ & $(()())$ \\
\hline Var2 & 0.56 & 19.72 & 0.56 & 5.78 & $70.69 \%$ & $(()())$ \\
\hline Var3 & 0.78 & 19.77 & 0.78 & 5.78 & $70.76 \%$ & $(()())$ \\
\hline Var4 & 1.49 & 19.61 & 1.49 & 5.66 & $71.14 \%$ & $(()())$ \\
\hline Var5 & 2.02 & 49.03 & 2.02 & 24.89 & $49.24 \%$ & $(()())$ \\
\hline Var6 & 0.59 & 19.69 & 0.59 & 5.70 & $71.05 \%$ & $(()())$ \\
\hline Var7 & 1.92 & 19.81 & 1.92 & 5.78 & $70.82 \%$ & $(()())$ \\
\hline Var8 & 0.34 & 19.73 & 0.34 & 5.72 & $71.01 \%$ & $(()())$ \\
\hline Var9 & 1.29 & 20.02 & 1.29 & 5.91 & $70.48 \%$ & $(()())$ \\
\hline Var10 & 1.91 & 19.69 & 1.91 & 5.76 & $70.75 \%$ & $(()())$ \\
\hline Var11 & 2.97 & 19.86 & 2.97 & 1.78 & $91.04 \%$ & $((()())(()()))$ \\
\hline Var12 & 4.75 & 19.72 & 4.75 & 3.88 & $80.32 \%$ & $((0)(())())$ \\
\hline Var13 & 4.96 & 19.84 & 4.96 & 3.73 & $81.20 \%$ & $((()())(())$ \\
\hline
\end{tabular}

Since it is prudent to investigate the computational advantage obtained by using a combination of the two modifications, $k W D$ and $k W R$, we have also devised an augmented modification, $k W D R$, which is the overall optimized version of the original $k$-Ward_MAT. Thus, $k W D R$ computes the micro-aggregation by recursive calls, and in each case it computes only the distance values advocated by Theorem 1 . The 
Table 3.7: Comparison between the original $k$-Ward_ $M A T, k W$, and the optimized $k W R$ using a dynamic threshold on the Census data set. In this case we used the value of $k=3$.

\begin{tabular}{|c|c|c|c|c|c|c|}
\hline \multirow{2}{*}{ Var } & \multicolumn{2}{|c|}{$k W$} & \multicolumn{4}{c|}{$k W R$} \\
\cline { 2 - 6 } & IL & Time & IL & Time & Improv. $(\%)$ & Recursion \\
\hline \hline Var1 & 0.131 & 42.44 & 0.131 & 30.78 & $27.47 \%$ & $(()())$ \\
\hline Var2 & 0.001 & 42.41 & 0.001 & 23.48 & $44.64 \%$ & $(()(()()))$ \\
\hline Var3 & 0.008 & 42.58 & 0.008 & 23.23 & $45.44 \%$ & $(()(()(()(()()))))$ \\
\hline Var4 & 0.005 & 42.03 & 0.005 & 23.33 & $44.49 \%$ & $(()(()(()(()()))))$ \\
\hline Var5 & 0.024 & 41.92 & 0.024 & 23.31 & $44.39 \%$ & $(()(()()))$ \\
\hline Var6 & 0.034 & 42.00 & 0.034 & 23.52 & $44.00 \%$ & $(()(()(()(()))))$ \\
\hline Var7 & 0.002 & 42.06 & 0.002 & 23.56 & $43.98 \%$ & $(()())$ \\
\hline Var8 & 0.443 & 41.69 & 0.443 & 23.55 & $43.51 \%$ & $(()())$ \\
\hline Var9 & 0.732 & 44.84 & 0.732 & 26.13 & $41.73 \%$ & $(()())$ \\
\hline Var10 & 0.003 & 47.02 & 0.005 & 29.63 & $36.98 \%$ & $(()(()(())))$ \\
\hline Var11 & 0.008 & 47.11 & 0.008 & 29.73 & $36.89 \%$ & $((()(()()))(()(()(()()))))$ \\
\hline Var12 & 0.004 & 47.31 & 0.004 & 29.94 & $36.72 \%$ & $(()(()(()()))$ \\
\hline Var13 & 0.005 & 48.34 & 0.005 & 30.83 & $36.22 \%$ & $(()(()(()())))$ \\
\hline
\end{tabular}

results of $k W D R$ are shown in Table 3.9. The reduction of time reaches up to $97.36 \%$ on the Tarragona data set by using a combination of $k W D$ and $k W R$ with a fixed threshold, while a combination of $k W D$ and $k W R$ with a dynamic threshold yields the $95.23 \%$ advantage on the Census data set.

To conclude, Figure 3.3 shows a comprehensive comparison between the original $k$-Ward_MAT, $k W D, k W R$, and $k W D R$ respectively using the Tarragona Data set. The power of our contribution is, thus, visually obvious.

\subsubsection{Results for Multi-variate MATs}

The previous experimental results display the value of the quantity $I L$, and the required computational time for uni-variate $M A T s$. We now demonstrate its applicability for multi-variate data, by considering several optimized versions of the $k$-Ward method on such data. Any multi-variate $M A T$ is designed to permit the simultaneous micro-aggregation of several variables, so as to yield a single $k$-partition for the entire 
Table 3.8: Comparison between the original $k$-Ward_ $M A T, k W$, and the optimized $k W D R$ which is a simultaneous combination of both $k W D$ and $k W R$ on the Tarragona data set. In this case we used the value of $k=3$ and a fixed threshold.

\begin{tabular}{|l|c|c|c|c|c|}
\hline \multirow{2}{*}{ Var } & \multicolumn{2}{|c|}{$k W$} & \multicolumn{3}{c|}{$k W D R$} \\
\cline { 2 - 5 } & IL & Time & IL & Time & Improv.(\%) \\
\hline \hline Var1 & 7.176 & 19.89 & 7.176 & 3.08 & $84.51 \%$ \\
\hline Var2 & 0.557 & 19.72 & 0.557 & 1.23 & $93.76 \%$ \\
\hline Var3 & 0.775 & 19.77 & 0.775 & 2.16 & $89.07 \%$ \\
\hline Var4 & 1.487 & 19.61 & 1.487 & 0.59 & $96.99 \%$ \\
\hline Var5 & 2.018 & 49.03 & 2.018 & 5.70 & $88.37 \%$ \\
\hline Var6 & 0.586 & 19.69 & 0.586 & 1.22 & $93.80 \%$ \\
\hline Var7 & 1.921 & 19.81 & 1.921 & 1.22 & $93.84 \%$ \\
\hline Var8 & 0.338 & 19.73 & 0.338 & 1.24 & $93.72 \%$ \\
\hline Var9 & 1.287 & 20.02 & 1.287 & 1.31 & $93.46 \%$ \\
\hline Var10 & 1.905 & 19.69 & 1.909 & 0.56 & $97.16 \%$ \\
\hline Var11 & 2.966 & 19.86 & 2.967 & 0.81 & $95.92 \%$ \\
\hline Var12 & 4.748 & 19.72 & 4.748 & 0.52 & $97.36 \%$ \\
\hline Var13 & 4.958 & 19.84 & 4.958 & 0.56 & $97.18 \%$ \\
\hline \hline
\end{tabular}

Table 3.9: Comparison between the original $k$-Ward_MAT, $k W$, and the optimized $k W D R$ which is a simultaneous combination of both $k W D$ and $k W R$ on the Census data set. In this case we used the value of $k=3$ and a dynamic threshold.

\begin{tabular}{|l|c|c|c|c|c|}
\hline \multirow{2}{*}{ Var } & \multicolumn{2}{|c|}{$k \mathrm{~W}$} & \multicolumn{3}{|c|}{$k W D R$} \\
\cline { 2 - 5 } & IL & Time & IL & Time & Improv.(\%) \\
\hline \hline Var1 & 0.131 & 42.44 & 0.131 & 13.39 & $68.45 \%$ \\
\hline Var2 & 0.001 & 42.41 & 0.001 & 2.42 & $94.29 \%$ \\
\hline Var3 & 0.008 & 42.58 & 0.008 & 2.03 & $95.23 \%$ \\
\hline Var4 & 0.005 & 42.03 & 0.005 & 2.20 & $94.77 \%$ \\
\hline Var5 & 0.024 & 41.92 & 0.024 & 2.52 & $93.99 \%$ \\
\hline Var6 & 0.034 & 42.00 & 0.034 & 2.27 & $94.60 \%$ \\
\hline Var7 & 0.002 & 42.06 & 0.002 & 4.28 & $89.82 \%$ \\
\hline Var8 & 0.443 & 41.69 & 0.443 & 4.39 & $89.47 \%$ \\
\hline Var9 & 0.732 & 44.84 & 0.732 & 5.67 & $87.36 \%$ \\
\hline Var10 & 0.003 & 47.02 & 0.005 & 5.70 & $87.88 \%$ \\
\hline Var11 & 0.008 & 47.11 & 0.011 & 4.59 & $90.26 \%$ \\
\hline Var12 & 0.004 & 47.31 & 0.008 & 6.55 & $86.16 \%$ \\
\hline Var13 & 0.005 & 48.34 & 0.009 & 6.92 & $85.68 \%$ \\
\hline \hline
\end{tabular}




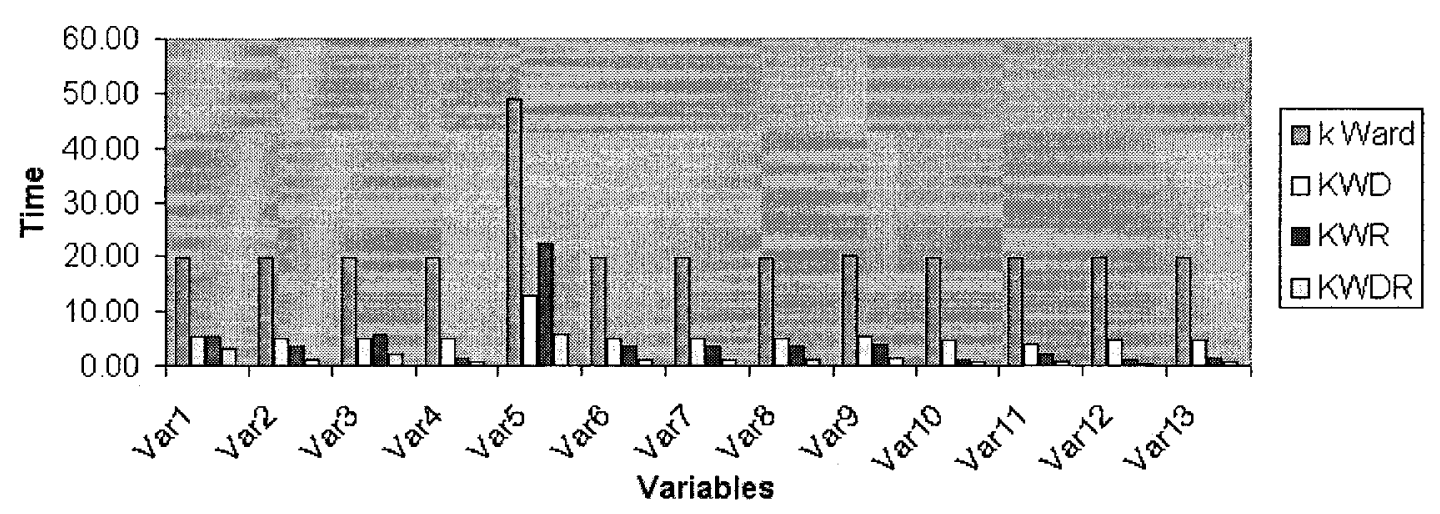

Figure 3.3: Comparison between $k$-Ward_MAT , $k W D, k W R$, and $k W D R$ using the Tarragona Data set. In this case we used the value of $k=3$

data set. As mentioned in Chapter 2, two approaches have been reported for such multi-variate MATs. The projected data $M A T$ is one in which the multi-variate data set is projected onto a single axis prior to invoking micro-aggregation. Since the projected data leads to a uni-variate data set, it can be sorted, after which any of the optimized versions referred to above can be used for micro-aggregation. As reported in [45], it is important, however that the multi-variate data set be normalized prior to the projection and the micro-aggregation phases, to prevent any of the undesired properties discussed there.

To avoid repetition, the results we present are necessarily brief.

Tables 3.10 and 3.11 respectively show a comprehensive comparison between the original $k$-Ward and the different optimized versions of the projected multi-variate $M A T$ on the Tarragona and Census data sets. The projection is done by using the sum of the $z$-scores of the variables, $Z S$, or the first principle component, FPC. The methods by which the data is preprocessed and examined are identical to those reported in $[43,45]$. The improvement in the micro-aggregation time by using the 
Table 3.10: Results for multi-variate $M A T s$ for the Tarragona data set obtained by projecting using the $F P C$ and the $Z S$ indices, In the table we compare the original $k$-Ward_MAT, $k W$, and the optimized $k W D, k W R$ using either a fixed threshold, $(k W R-F)$, or a dynamic threshold, $(k W R-D)$, and $k W D R$ which is a simultaneous combination of both $k W D$ and $k W R-D$, by setting $k=3$.

\begin{tabular}{|c|l|c|c|c|c|}
\hline Projection & \multirow{2}{*}{ Method } & \multicolumn{4}{|c|}{ Tarragona Data Set } \\
\cline { 3 - 6 } & & IL & Time & Improv.(\%) & Recursion \\
\hline \hline & $k W$ & 30.13 & 22.43 & & \\
\hline & $k W D$ & 30.13 & 4.89 & 78.20 & \\
\hline$S Z$ & $k W R-F$ & 30.13 & 3.27 & 85.42 & $(()(()))$ \\
\hline & $k W R-D$ & 30.13 & 10.06 & 55.15 & $(()())$ \\
\hline & $k W D R$ & 30.13 & 6.39 & 71.51 & $(()())$ \\
\hline \hline & $k W$ & 25.35 & 28.32 & & \\
\hline & $k W D$ & 25.35 & 10.86 & 61.65 & \\
\hline$F P C$ & $k W R-F$ & 25.35 & 3.28 & 88.42 & $(()(()())$ \\
\hline & $k W R-D$ & 25.35 & 5.81 & 79.48 & $(()())$ \\
\hline & $k W D R$ & 25.35 & 2.09 & 92.62 & $(()())$ \\
\hline
\end{tabular}

Table 3.11: Results for multi-variate MATs for the Census data set obtained by projecting using the $F P C$ and the $Z S$ indices, In the table we compare the original $k$-Ward_MAT, $k W$, and the optimized $k W D, k W R$ using either a fixed threshold, $(k W R-F)$, or a dynamic threshold, $(k W R-D)$, and $k W D R$ which is a simultaneous combination of both $k W D$ and $k W R-D$, by setting $k=3$.

\begin{tabular}{|c|l|c|c|c|c|}
\hline Projection & \multirow{2}{*}{ Method } & \multicolumn{3}{|c|}{ Census Data Set } \\
\cline { 3 - 6 } & & IL & Time & Improv.(\%) & Recursion \\
\hline \hline & $k W$ & 28.58 & 48.82 & & \\
\hline & $k W D$ & 28.58 & 0.86 & 98.24 & \\
\hline$S Z$ & $k W R-F$ & 28.61 & 7.33 & 84.99 & $(()(()(()((()())(()((()())(()(()(()(()()))))))))))$ \\
\hline & $k W R-D$ & 28.58 & 11.64 & 76.16 & $(()(()(()(()(()(()(()())))))))$ \\
\hline & $k W D R$ & 28.58 & 6.56 & 86.56 & $(()(()(()(()(()(()(()())))))))$ \\
\hline \hline & $k W$ & 29.54 & 61.813 & & \\
\hline & $k W D$ & 29.54 & 21.63 & 65.01 & $((((()(()()))(()(()(()()))))())())$ \\
\hline$F P C$ & $k W R-F$ & 29.54 & 6.72 & 89.13 & $(((()())(())())(())$ \\
\hline & $k W R-D$ & 29.54 & 7.72 & 87.51 & $((((())())(())())())$ \\
\hline & $k W D R$ & 29.54 & 2.61 & 95.78 &
\end{tabular}


$k W D$ was as high as $78.20 \%$ and $61.65 \%$ on the $Z S$ and $F P C$ projections of the Tarragona data set, respectively. Similarly, the time improvement reached up to $98.24 \%$ and $65.01 \%$ on the $Z S$ and the $F P C$ projection of the Census data set. The $k W R$ method was also applied by using a fixed threshold, $k W R-F$, or a dynamic threshold, $k W R-D$ on the projected data sets as explained in Section 3.4.2. In the case of $k W R-F$, the threshold value was set to 0.8 for the projected Tarragona data set, and to 0.3 for the projected Census data set. These "optimal" setup parameters were obtained by trial-and-error testing. Generally speaking, the time improvement using the $k W R-F$ was more than that obtained by using the $k W R-D$-it reached up to $89.13 \%$ by using $k W R-F$ on the $F P C$ projection for the Census data set, and up to $87.61 \%$ by using the $k W R-D$ on the $F P C$ projection for the same data. Unfortunately, the value of the $I L$ was sometimes affected by invoking $k W R-F$, (as can be observed in Table 3.11 for the projected $Z S$ of the Census data sets), while the value of the $I L$ remains the same by using the $k W R-D$. Finally, it is important to mention that the combination of the $k W D$ and $k W R-D$ increases the improvement of the required time to $95.78 \%$ on the $F P C$ projection of the Census data set without any noticeable change in the value of the $I L$. It is, thus, clear that the enhanced versions of such proposed schemes are applicable to the processing of multi-variate data sets, by projecting them onto a single axis using the FPC or $Z S$ methods.

\subsection{Conclusion}

In this chapter, we have considered the problem of securing a statistical database. We have resorted to the well-known micro-aggregation technique, and in particular, considered the $k$-Ward strategy introduced in [139] and utilized in the state-of-theart methods [45]. The latter scheme coalesces the sorted data attribute values into groups, and on being queried, reports the means of the corresponding groups. We have shown that such a scheme, as reported in the literature, can be enhanced by minimizing the computations done in evaluating the between-class distance matrix and 
by recursively partitioning the data set before $k$-Ward strategy is invoked. When the latter is invoked on the "primitive" sub-groups which terminate the recursion. Our experimental results, done on two benchmark data sets, report a marked improvement. While the $I L$ is comparable to the $k$-Ward_MAT proposed by Domingo-Ferrer et.al. [45], the computations required to achieve this loss is a fraction of the computations required in the latter. This provides a computational advantage that sometimes exceeds $80 \%$ if one method is used by itself, and more than $90 \%$ if both enhancements are invoked simultaneously.

Finally, although all these methods, were formally proven to be efficient for univariate data sets, we were also able to demonstrate the power of the schemes for multi-variate data. This was achieved by using a projection mechanism, such as the $F P C$ or $Z S$, and then invoking the $M A T$ on the projected data. Again, the favorableness of the result demonstrated the applicability of the methods. 


\section{Chapter 4}

\section{A Fixed Structure LA-Based MAT}

\subsection{Introduction}

We consider the $M A P$ which involves partitioning a set of individual records in a micro-data file into a number of mutually exclusive and exhaustive groups. This problem, which seeks for the best partition of the micro-data file, is known to be NP-hard and has been tackled using many heuristic solutions. In this chapter ${ }^{1}$ we present the first reported Learning Automata $(L A)$ based solution to this problem. The $L A$ modify a fixed-structure solution to the Equi-Partitioning Problem (EPP) to solve the $M A P$. The newly proposed method competes in a superior manner against the state-of-the-art methods not only from the $I L$ perspective, but when it concerns a criterion involving a combination of the $I L$ and the $D R$. The scheme has been implemented, tested and evaluated for different real-life and simulated data sets. The results clearly demonstrate the applicability of $L A$ to the $M A P$, and its ability to yield a solution that obtains the best trade-off between $I L$ and $D R$ when compared to the state-of-the-art.

\footnotetext{
${ }^{1}$ A preliminary version of some of the results from this chapter appeared in the Proceedings of PSD'06, the Privacy in Statistical Databases Conference, in Rome, Italy, in December 2006 [64]. The journal version of these results is currently under review.
} 
The structure of this chapter is as follows: Section 4.2 initially provides a basic introduction to the field of $L A$, and also explains how $L A$ principles can be used to formulate a solution to the $M A P$. Section 4.3 presents the Object Migrating Micro-aggregated Automaton both informally and algorithmically. In Section 4.4 we describe how we compute the composite score, which we refer to as the Scoring In$\operatorname{dex}(S I)$, used for ranking the $M D A V$ and the new $L A$-based methods with certain specific parameterizations. Experimental results using real-life benchmark and simulated data sets, are discussed in Section 6.3. Finally, Section 6.4 presents the overall conclusions of this work.

\subsubsection{Contribution of the Chapter}

As mentioned in the literature, this problem in its multi-variate setting is known to be $N P$-hard [103], and has been tackled using different approaches such as hierarchical clustering [45, 91, 92], genetic algorithms [45, 91, 92, 128], graph theory [74, 84], and fuzzy clustering $[57,133]$. All the heuristic MATs seek to minimize the value of the $I L$. Observe that minimizing the $I L$, though important, is difficult to enforce, essentially because any $S D C$ strategy is primarily intended to enhance the security of the data. Indeed, the definition of optimality for an $S D C$ is defined in the literature as being equivalent to offer the best trade-off between the $I L$ and $D R[27,93]$. In this vein, the recent development of MATs [60] leaves the researcher no excuse to circumvent the problem of trying to reduce the value of the $I L$ as much as possible [49].

As argued by most researchers, maintaining a happy medium between the $I L$ and $D R$ is not trivial. Indeed, even the question of how such a compromise can be attained has not been fully investigated. In this chapter, we shall argue that a good $M A T$ should be capable of minimizing the $I L$, and yet be able to attain to suitable value for a well-defined composite measure. One such measure, which we refer to as the $S I$, is a linear combination of the $I L$ and $D R$. Our aim is to find a good strategy to optimize the latter. 
In general, minimizing the $I L$ directly follows the goal of maximizing the similarity between the records in each group. The latter, in turn, can be maximized by using two criteria, namely that of requiring the total $S S E$ to be as low as possible and, simultaneously, preserving the relationships between the individual records in the micro-data file. This is precisely the rationale of our current methodology. In this chapter, we achieve this by attempting to solve the problem using the principles of $L A$. We have described one such $L A$ called the Object Migrating Micro-aggregated Automaton $(O M M A)$, which is capable of grouping similar records, and simultaneously differentiating records which do not belong together. The unique strength of this methodology lies in its applicability to cluster multi-variate data sets without resorting to projection in any preferred direction. The chapter, thus, demonstrates the power of $L A$ to minimize the $I L$, leading to results comparable to those obtained from the best available heuristic methods for micro-aggregation such as the Maximum Distance to Average Vector $(M D A V)[60]$ and the Minimum Spanning Tree Partitioning Algorithm (MST) [84]. The remarkable reduction obtained in the $I L$ can be noticed when micro-aggregating multi-variate data (which sometimes exceeds $11 \%$ when compared to the $M D A V$ and $M S T)$, and it renders the contribution of this chapter significant. Apart from this, the $O M M A$ also yields the best reported values for the above mentioned index, the $S I$. We argue that the applicability of $L A$ to the $M A T$ provides a promising strategy to effectively protect sensitive data in the micro-data file not only based on minimizing the value of the $I L$ but also on offering the best trade-off between the $I L$ and $D R$. The limitations and possible extensions of the $O M M A$ are explained in a subsequent section.

It should be mentioned that by using our strategy, the $O M M A$ can actually be applied to many types of attributes - continuous, ordinal or nominal. The only difference that we foresee will be in designing the aggregation operator (i.e., one which yields the "mean" for the continuous data, or the median for categorical data), which is computed for each group, and which is used to replace the original records. But the main strength of the methodology lies in its applicability to cluster multi-variate data sets without resorting to projection in any preferred direction. This is because 
the scheme does not merely utilize the distance between the individual records, but it also attempts to keep the $S S E$ minimum.

\subsection{Learning Automata $(L A)$}

Our solution to the MAP involves $L A . L A$ have been used to model biological learning systems and to learn the optimal action which a random environment offers. The learning is accomplished by actually interacting with an environment and processing its responses to the actions that are chosen, while gradually converging toward an ultimate goal. There are various applications that use $L A$ including parameter optimization, statistical decision making, telephone routing, pattern recognition, game playing, natural language processing, modeling biological learning systems, string taxonomy, detection and tracking, distributing the process of a parallel application, routing in communication networks and object partitioning $[71,75,95,98,99,105,109,111,112,113,147]$. Since the literature on $L A$ is extensive, we refer the reader to $[98]$ for a good survey.

The functionality of the $L A$ can be described as a sequence of repetitive feedback cycles. The feedback cycle involves two entities, the Random Environment and the $L A$. During each cycle the automaton chooses an action, which triggers a response from the Environment, and uses the received response - that can be either a reward or a penalty- with the knowledge gained from the previous cycles to determine which is the next action to be chosen. By the process of learning, the automaton adapts itself to the Environment and determines the optimal action, i.e., the action which has the minimum penalty probability, or has a maximum reward probability.

Incorporating $L A$ in any application domain is an evidence of the power of the philosophy. Basically, $L A$ learn from the random environment. The actual technique involved in applying the $L A$ philosophy in the different applications involves modeling the actions, simulating the transforming functions, and representing the system's output in order to have reward or penalty responses. This is where the creativity of 
the researcher becomes apparent.

Stochastic $L A$ can be classified into two main classes:

1. Fixed Structure Stochastic Automata ( FSSA): These FSSA have the property that their transition and output functions do not change with time. These $L A$ seem to possess powerful properties useful for solving different NP-hard problems, as we will show in this chapter.

2. Variable Structure Stochastic Automata (VSSA): These VSSA have a dynamically changing structure, because their transition and output matrices are time varying. In practice, however, they are merely defined in terms of action probability updating rules which are either of a continuous or discrete nature $[3,110]$. Automata with a variable structure are generally much faster in their convergence.

The basic idea used to solve the $M A P$ is based on a sub-class of $L A$ solutions that has been used to solve the object partitioning problem [71, 111, 112]. As documented in the literature, the object partitioning problem involves partitioning a set of $|\mathbb{P}|$ objects into $|\mathbb{N}|$ groups or classes, where the main aim is to partition the objects into groups that mimic an underlying unknown grouping. In other words, the objects which are accessed together must reside in the same group [112]. In the special case when all the groups are required to contain the same number of objects, the problem is also referred to as the EPP. Many solutions involving $L A$ have been proposed to solve the EPP, but the most efficient algorithm is the Object Migrating Automaton $(O M A)$ [112]. The latter was first proposed by Oommen and Ma [112], and some modifications to the original algorithm were added by Gale et.al. [71] to create the Adaptive Clustering Algorithm (ACA).

For the rest of this section, in all brevity, we discuss the fundamental of $L A$ and present the algorithm due to Oommen and Ma [112| and the modified version by Gale et.al.. 


\subsubsection{Similarities Between the MAP and the EPP}

The idea behind using $L A$ as an $M A T$ comes from the elegance of using $L A$ in solving the EPP. LA possess many attractive features that make them applicable to the $M A T$ such as simplicity, ease of implementation, and the ability to study the relation between the individual objects/records without computing any statistical queries. We will show that the similarity between the EPP and the MAP render $L A$ as one of the promising candidate tools to solve the latter. This is because:

- The EPP and MAP are NP-hard problems, primarily due to the exponential growth in the number of partitions of records/objects.

- The EPP dictates that each partition must have the same number of objects, which is a condition analogous to the one associated with the $M A P$ for the fixed-size micro-aggregation criterion, which states that each group must have approximately the same number of records.

- The EPP and MAP seek to partition the records/objects into groups that mimic the underlying unknown groups. In the case of the $E P P$, the objects which are accessed together more frequently by a random sequence of queries are said to be in the same partition. As opposed to this, in the $M A P$, the records which are similar to each other, are required to be in the same group so as to maximize the within-group and to minimize the between-group similarities.

\subsubsection{Fundamentals}

An FSSA is a quintuple $(\underline{\alpha}, \underline{\Phi}, \underline{\beta}, \mathrm{F}, \mathrm{G})$ where

- $\underline{\alpha}=\left\{\alpha_{1}, \ldots, \alpha_{R}\right\}$ is the set of actions that, it must choose from.

- $\underline{\Phi}=\left\{\phi_{1}, \ldots, \phi_{S}\right\}$ is a set of states. 
- $\underline{\beta}=\{0,1\}$ is its set of inputs. The " 1 " represents a penalty, while the " 0 " represents a reward.

- $F$ is a map from $\Phi \times \beta$ to $\Phi$. It defines the transition of the internal state of the automaton on receiving an input. $F$ may be stochastic.

- $G$ is a map from $\Phi$ to $\alpha$, and it determines the action taken by the automaton if it is in a given state. With no loss of generality, $G$ is deterministic.

As discussed above, the automaton is offered a set of actions, and it is constrained to choose one of them. When an action is chosen, the Environment gives out a response $\beta(n)$ at a time " $n$ ". The automaton is either penalized or rewarded with an unknown probability $c_{i}$ or $1-c_{i}$, respectively. On the basis of the response $\beta(n)$, the state of the automaton $\phi(n)$ is updated and a new action is chosen at $(\mathrm{n}+1)$. The penalty probability $c_{i}$ satisfies:

$$
c_{i}=\operatorname{Pr}\left[\beta(n)=1 \mid \alpha(n)=\alpha_{i}\right] \quad(i=1,2, \ldots, R) .
$$

\subsubsection{Object Migrating Automaton $(O M A)$}

The Object Migrating Automaton $(O M A)$ is an ergodic automaton that has $R$ actions $\left\{\alpha_{1}, \ldots, \alpha_{R}\right\}$ representing the possible underlying classes. Each action $\alpha_{i}$ has its own set of states $\left\{\phi_{i 1}, \phi_{i 2} \ldots, \phi_{i M}\right\}$, where $M$ is the depth of memory, and $1 \leq i \leq R$ represents the number of classes. $\phi_{i 1}$ is called the most internal state and $\phi_{i M}$ is the most external state.

A set of $\mathrm{W}$ physical objects $\left\{A_{1}, A_{2}, \ldots, A_{W}\right\}$ is accessed by a random stream of queries, and the objects are to be partitioned into groups so that the frequently jointly-accessed objects are clustered together. The $O M A$ utilizes $W$ abstract objects $\left\{O_{1}, O_{2}, \ldots, O_{W}\right\}$ instead of migrating the physical objects. Each abstract object is assigned to a state belonging to an initial random group but in its boundary state. The objects within the automaton move from one action to another, and so, in this 
case, all the $W$ abstract objects move around in the automaton. If the abstract objects $O_{i}$ and $O_{j}$ are in the action $\alpha_{h}$, and the request accesses $\left\langle A_{i}, A_{j}\right\rangle$, then the $O M A$ will be rewarded by moving them towards the most internal state $\phi_{h 1}$. But a penalty arises if the abstract objects $O_{i}$ and $O_{j}$ are in different classes, say $\alpha_{h}$ and $\alpha_{g}$, respectively. Assuming $O_{i}$ is in $\zeta_{i} \varepsilon\left\{\phi_{h 1}, \phi_{h 2}, \ldots, \phi_{h M}\right\}$ and $O_{j}$ is in $\zeta_{j} \varepsilon\left\{\phi_{g 1}, \phi_{g 2}, \ldots, \phi_{g M}\right\}$, they will be moved as follows:

- If $\zeta_{i} \neq \phi_{h M}$ and $\zeta_{j} \neq \phi_{g M}, O_{i}$ and $O_{j}$ are moved one state toward $\phi_{h M}$ and $\phi_{g M}$, respectively.

- If exactly one of them is in the boundary state, the object which is not in the boundary state is moved towards its boundary state.

- If both of them are in their boundary states, one of them, say $O_{i}$ is moved to the boundary state of the other object $\phi_{g M}$. In addition, the closest object to them is moved to the boundary state $\phi_{h M}$, so as to preserve an equal number of objects in each group.

It is important to point out that the random stream of queries contains information about an optimal partition, and the $O M A$ attempts to converge to it. The automaton is said to have converged when all the objects associated with a class are in the deepest (or second deepest) most-internal state.

The $O M A$ can be improved by the following: Assume a pair of objects $\left\langle O_{i}, O_{j}\right\rangle$ is accessed, where $O_{i}$ is in the boundary state, while $O_{j}$ is in a non-boundary state. In this case, a general check should be made to find another object, in the boundary state of the partition containing $O_{j}$. If there is an object, then swapping is done between this object and $O_{i}$ in order to bring the two accessed objects into the same partition. In turn, instead of waiting for a long time to have these accessed objects in the same partition, the convergence speed can be increased by swapping the objects into the right partitions.

The formal algorithm for the $O M A$ can be found in $[71,112]$; it is shown in 


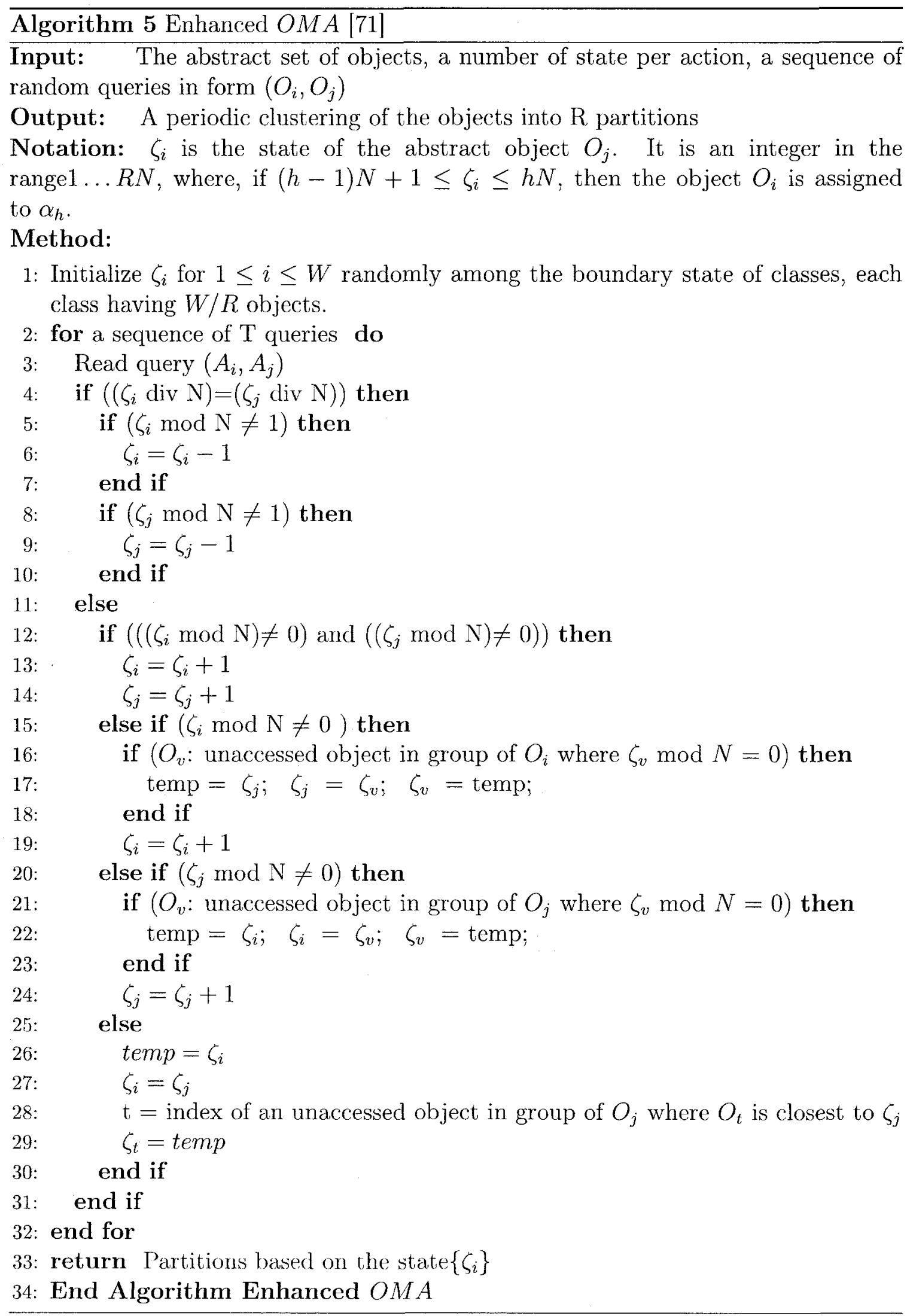


Algorithm 5.

\subsubsection{Restrictions of the $O M A$ to the $M A P$}

The reported instances of the $O M A$ are not directly applicable for the $M A P$. To develop our solution, we highlight the main restrictions, and the necessary enhancements which must be added to the $O M A$ in order for it to be useful in our present application domain.

- In case of the $M A P$, the user does not have access to the stream of random queries. Rather, the only available data is the set of individual records stored in the micro-data file. It is thus apparent that we have to artificially "generate" a sequence of "queries" (or pairs) which can be used to operate on a machine similar to the $O M A$. The above restriction has a "two-edged" implication. First of all, in the $E P P$, the user usually requests the system to obtain a query pair of the form $\left\langle O_{i}, O_{j}\right\rangle$. However, in the $M A P$, it is the responsibility of the user to determine which records are similar or dissimilar, and this determination is a problem to be solved in its own right. Secondly, the placement of the objects in the automaton and the stream of random queries, together, serve to either reward or penalize the automaton. However, in the case of the $M A P$, the question of obtaining a reward/penalty response is not provided by the user, but it has to be inferred. This again has to be solved.

- Unlike the $E P P$, which has no way of penalizing "non accessed elements", a solution to the MAP must develop a strategy for penalizing such records by considering how similar the records within the same groups are. Clearly, this is superfluous for the EPP because, in that problem, the automaton is absolutely dependent on the user's queries. In the present problem, it is crucial that an automaton can quantify how fitting a record is for any given group.

- The optimal partition for the EPP yields crucial information in the stream of random queries. As opposed to this, in the context of the $M A P$, the system 
has no notion of how to characterize the optimal partition. This renders the problem of adapting the $O M A$ to solve the $M A P$ more difficult.

- In the same vein, the definition of the optimal partition for the $E P P$ is quite different from that of the corresponding solution for the $M A P$. In the case of the $E P P$, all objects which are accessed together more frequently should be in the same partition, while in the $M A P$ all similar records should be in the same group.

- The criteria which are used to reward and penalize the automaton in the EPP is quite unlike the one used for the $M A P$. In the EPP, the automaton is rewarded or penalized based on the (unknown) probability of any two objects being jointly accessed. But in the context of the $M A P$, the automaton is reward or penalized by conducting a comprehensive study of the relation between the individual records, quantified by the various error criteria, such as the distance between each record and the other records in the micro-data file, and more importantly, the overall $S S E$.

- Although the EPP and $M A P$ utilize analogous rules for a reward phenomenon, as we shall see, they differ in performing the penalty rules. In case of the EPP, the automaton enforces the rule that the pertinent object migrates, if and only if at least one of the accessed objects is at the boundary state of the different partitions. As opposed to this, in the MAP, the automaton enforces the rule that the records are migrated to another group whenever migrating the object reduces the overall $S S E$.

- The automaton used to solve the EPP is said to have converged, when all the objects are found in the most (or the last two) internal states of each partition. However, we propose that the convergence in the $M A P$ occur when the measure of the $I L$ is unchanged. 


\subsection{Object Migrating Micro-aggregated Automaton $(O M M A)$}

In this section, we define the $O M M A$ as an 7 -tuple as: $(\mathbb{U}, \underline{\Phi}, \underline{\alpha}, \underline{\beta}, \mathbb{Q}, \mathbb{G}, \mathbb{L})$, where

- $\mathbb{U}=\left\{U_{1}, U_{2}, \ldots, U_{n}\right\}$ is the micro-data file.

- $\underline{\Phi}=\left\{\phi_{1}, \phi_{2}, \ldots, \phi_{h M}\right\}$ is the set of states.

- $\underline{\alpha}=\left\{\alpha_{1}, \alpha_{2}, \ldots, \alpha_{h}\right\}$ is the set of $h$ actions, each representing a group into which the records of $\mathbb{U}$ must fall.

- $\underline{\beta}=\{0,1\}$ is its set of inputs. The "1" represents a penalty, while the " 0 " represents a reward.

- $\mathbb{Q}$ is the transition function, which specifies how the records should move between the various states. This function is quite involved and will be explained in detail presently.

- The function $\mathbb{G}$ partitions the set of states for the groups. For each group, $\alpha_{j}$, there is a set of states $\left\{\phi_{(j-1) M+1, \ldots, \phi_{j M}}\right\}$, where $M^{2}$ is the depth of memory. Thus,

$$
G\left(\phi_{i}\right)=\alpha_{j} \quad(j-1) M+1 \leq i \leq j M
$$

This means that the record in the automaton chooses $\alpha_{1}$, if it is in any of the first $M$ states, and that it chooses $\alpha_{2}$ if it is in any of the states from $\phi_{M+1}$ to $\phi_{2 M}$, etc. We assume that $\phi_{(j-1) M+1}$ is the most internal state of group $\alpha_{j}$,

\footnotetext{
${ }^{2}$ Generally speaking, the depth of the memory, $M$ in the $L A$ could play an important role in determining the accuracy of the LA, while the eignevalues of the underlying chain would determine the rate of convergence. In practice, the LA is assumed to have converged when all objects are in the most internal states in each partition. To the best of our knowledge we are not aware of any method used to determine the best value for $M$ except the trial-and-error approach. In our case, since the convergence criterion depends on attaining to a non-increasing value of $I L$, it is not implicitly tied to the value $M$. Thus, in the case of the $O M M A, M$ seems to have no effect on the computational time and the algorithm's convergence and accuracy .
} 
and that $\phi_{j M}$ is the boundary state. These are called the state of "Maximum Certainty" and "Minimum Certainty", respectively.

- $\mathbb{L}$ is the similarity list specifying the records deemed to be collectively similar. It is stored as a list of tuples of the form $\left\langle R_{i}, R_{j}\right\rangle$, where the records included are those for which the similarity index is greater than or equal to a predefined threshold, $\theta$.
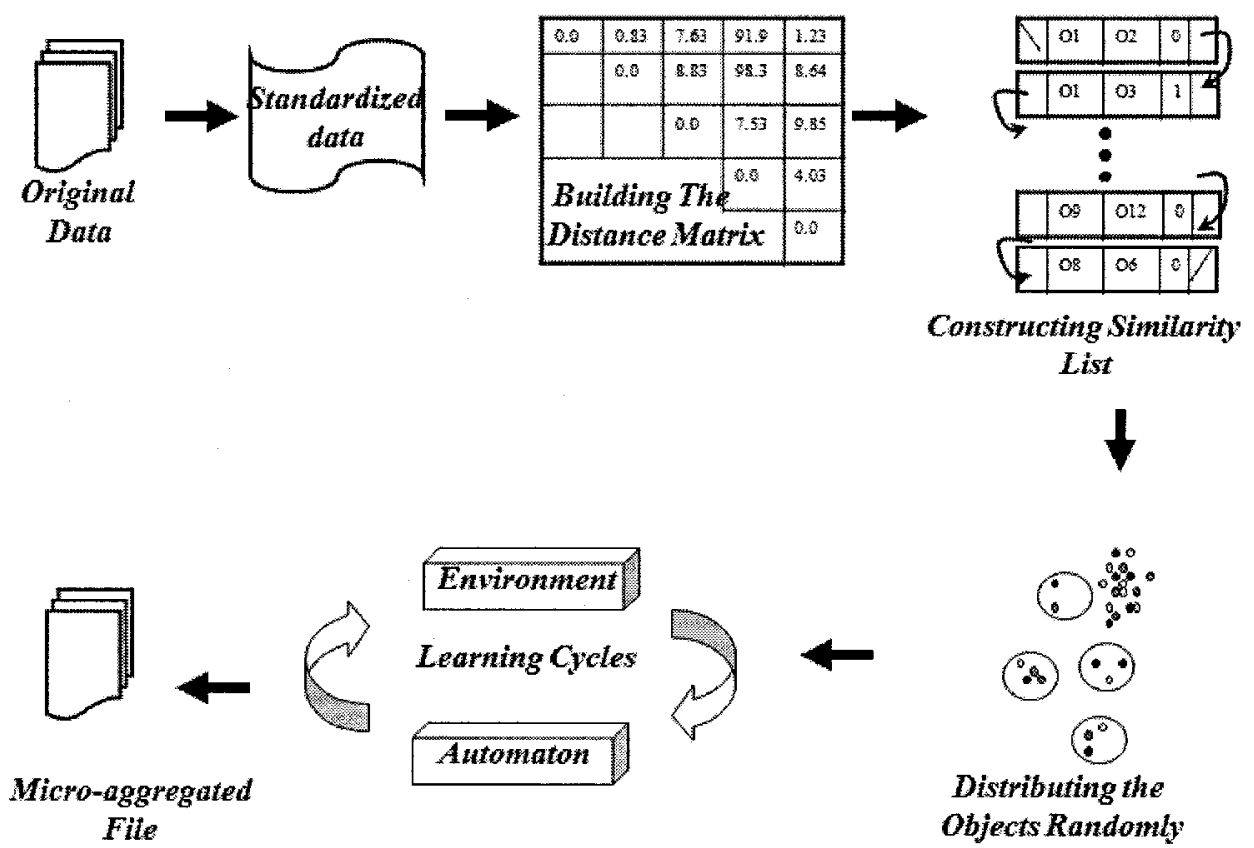

Figure 4.1: The $O M M A$ process used to generate the micro-aggregated file.

Before we explain in greater detail the $O M M A$, it is worth mentioning that this new scheme is applicable when the number of individuals in the micro-data file is an exact multiple of the minimum group size $k$. When the number of records is not an exact multiple of $k$, we propose that the $M A T$ be invoked for a marginally smaller file (by removing a few elements in the set), and then including them subsequently in the appropriate partitions - after the $M A T$ process. 
The overall model for this application domain is given in Figure 4.1 which shows that the $O M M A$ process consists of three phases involved in generating the microaggregated data file as follows:

1. Loading and standardizing the micro-data file.

The variables encountered in the micro-data file have to be standardized prior to the micro-aggregation process. The standardization is performed by replacing the individual values, $x_{i}$, for a specific variable, $V_{j}$, by $\left(x_{i}-\bar{X}_{j}\right) / \sigma_{j}$, where $\bar{X}_{j}$ and $\sigma_{j}$ are, respectively, the average and the standard deviation of the values taken by the variable $V_{j}$. The importance of this phase is evident when we consider multi-variate micro-data, because having standardized variables prior to the micro-aggregation process removes many undesirable properties, and yields equal weights for all the variables encountered in the micro-data file.

2. Constructing the similarity list that simulates the similarities between microrecords.

The similarity list, $\mathbb{L}$, simulates the probability of any two records being together in the same group or not. This simulation is based on the real distance value between every pair of records. Therefore, building the similarity list requires creating the symmetric $N \times N$ distance matrix. Each entry in the matrix is computed using the Euclidean metric between the two records in question as follows, $D\left(R_{i}, R_{j}\right)=\sqrt{\sum_{d=1}^{p}\left(R_{i d}-R_{j d}\right)^{2}}$, where $D\left(R_{i}, R_{j}\right)$ is the computed distance between the two records $R_{i}$ and $R_{j}$, and the entry is placed in the $i^{\text {th }}$ row and the $j^{\text {th }}$ column. The rationale behind building the similarity matrix is to simulate the probability of these two records being together in the same group, and this, in turn, is used in constructing the similarity list. It is worth mentioning that constructing the similarity list is done only once, and this can be considered as a preprocessing operation, and hence the OMMA method is 
suitable for small and moderate-sized data sets ${ }^{3}$. Thereafter, it requires comparing the elements of the distance matrix with a certain user-defined threshold, $\theta$. The question of determining $\theta$ is open. As it stands now, we have used the following single heuristic: $\theta$ is quite simply assigned to be related to the value of the difference between the largest and smallest values in the distance matrix. More explicitly,

$$
\theta=\frac{1}{2}\left[\operatorname{Max}_{i, j} D(i, j)-\operatorname{Min}_{i, j} D(i, j)\right]
$$

where $D(i, j)$ is the computed distance value between $R_{i}$ and $R_{j}$. In general, this threshold value is used to make the scheme arrive at an efficient grouping. Finally, if the distance value between $R_{i}$ and $R_{j}$ is less than or equal to $\theta$, we will add a tuple $\left\langle R_{i}, R_{j}\right\rangle$ to the similarity list. By computing the quantities $D\left(R_{i}, R_{j}\right)$ between each pair of records in the micro-data file, and by systematically utilizing the similarity list structure, the automaton must adaptively learn how to group the records effectively.

3. Performing the learning cycle, which is where the MAP is solved.

The learning phase is the core of the clustering, and the OMMA model is initialized by placing all the records at the boundary state of their initially randomly-chosen groups. This indicates that the $O M M A$ is initially uncertain of the placement of the records, because the different states within a given group quantify the measure of certainty that the scheme has for a given record belonging to that group. As the learning cycle proceeds, similar records will be rewarded for their being together in the same group, and they will be penalized by cither moving toward their boundary state, or to another group. After the randomly distribution of the micro-records, a certain index will be assigned to each record to specify the state of group it belong to, and the value of the SSE for each group will be calculated to quantify the homogenousity factor of that

\footnotetext{
${ }^{3}$ The associated computational time can be reduced by using the blocking technique |127|. Since the $O M M A$ runs in a very reasonable time in all the small and medium-sized cases, we have not resorted to the blocking technique in our implementation. The concept of using a hierarchy of $O M M A$ for large data sets has to be investigated.
} 
group.

The OMMA moves into its main learning loop by setting the old value of the $I L$ to be equal to $\infty$ (a large positive number), and by then processing the constructed similarity list, $\mathbb{L}$, one tuple at a time. It is worth mentioning that the length of the similarity list might be decreased with time by deleting the tuple after being sure that, although these two records are close to each other, their being in the same group will lead to increase the total SSE. The latter consideration is relevant since the $S S E$ functionally determines the value of the $I L^{4}$.

The list $\mathbb{L}$ is now traversed repeatedly, and similar records in the tuples are processed. If they are both assigned to the same group, the automaton is rewarded. However, if they are assigned to distinct groups, the automaton is penalized. After $\mathbb{L}$ has been processed, we compare the newly computed value of the $I L, I L_{n e w}$, with the old value, $I L_{\text {old }}$, produced in the previous cycle. If $I L_{n e w}<I L_{\text {old }}$, the learning phase continues by entering the next learning cycle. But, if $I L_{n e w}=I L_{o l d}$, the learning phase terminates and the micro-aggregated file is generated. It should be mentioned that $I L_{n e w}$ cannot be greater than $I L_{\text {old }}$, because the records will move, if and only if, the SSE remains the same or is reduced. Clearly, the value of $I L$ is non-increasing.

We now describe the actual transitions represented by $\mathbb{Q}$ for each of these operations.

\section{(a) Transitions for Rewards}

On being rewarded, since the records $R_{i}$ and $R_{j}$ are in the same group, say $\alpha_{u}$, both of them are moved toward the most internal state of that group, one step at a time. See Figure 4.2 and Algorithm 7.

(b) Transitions for Penalties

This case is encountered when two similar records, $R_{i}$ and $R_{j}$, are allocated in distinct groups. say, $\alpha_{u}$ and $\alpha_{v}$ respectively (i.e., $R_{i}$ is in state $\zeta_{i}$, where

\footnotetext{
${ }^{4}$ The clustering in the $O M M A$ is achieved based on two conflicting criteria - minimizing the distance between the records, and maintaining the total $S S E$ to be as small as possible.
} 
$\zeta_{i} \epsilon\left\{\Phi_{(u-1) M+1}, \ldots, \phi_{u M}\right\}$, and $R_{j}$ is in state $\zeta_{j}$, where $\left.\zeta_{j} \epsilon\left\{\Phi_{(v-1) M+1}, \ldots, \phi_{v M}\right\}\right)$. In this case, they are moved as follows:

- Case 1: If both $\zeta_{i}$ and $\zeta_{j}$ are not the boundary states $\phi_{u M}$ and $\phi_{v M}$, respectively, $R_{i}$ and $R_{j}$ are moved one state toward the corresponding boundary state. See Figure 4.3 and Algorithm 8 .

- Case 2: This occurs when at least one of $R_{i}$ or $R_{j}$ is at the boundary state of Minimum Certainty, say $\zeta_{j}=\phi_{v M}$. Studying the effect of migrating any record $R_{x}$ in $\alpha_{u}$ to $R_{j}$, under the condition that $R_{x} \neq R_{i}$, requires investigating the effect of the potential moves on the summation of the $S S E_{\alpha_{u}}$ and $S S E_{\alpha_{v}}$, which we shall refer to as $S S E_{\alpha_{u v}}$. Here we need to consider the $k-1$ different swapping possibilities. If the new value of $S S E_{\alpha_{u v}}$ is less than or equal to the previous value of $S S E_{\alpha_{u v}}$, we have to physically swap the chosen record $R_{x}$, which leads to the minimum $S S E_{\alpha_{w v}}$ value with $R_{j}$, and proceed to update the values of the $S S E$ for both groups. Subsequently, we assign both $R_{j}$ and $R_{x}$ to the boundary state of $\alpha_{u}$ and $\alpha_{v}$, respectively. Otherwise, the tuple $\left\langle R_{i}, R_{j}\right\rangle$ will be deleted from the similarity list. This is clarified in Figure 4.4 and Algorithm 9.

- Case 3: If both $R_{i}$ and $R_{j}$ are at the boundary states of their different groups, say $\zeta_{i}=\phi_{u M}$ and $\zeta_{j}=\phi_{v M}$, we have to study all the different possibilities of swapping any record $R_{x}$, under the condition that $R_{x} \neq R_{i}$ and $R_{x} \neq R_{j}$, in $\alpha_{u}$ or $\alpha_{v}$ with $R_{j}$ or $R_{i}$, respectively, on the $S S E_{\alpha_{u v}}$. We then choose the option that leads to reducing the value of $S S E_{\alpha_{w v}}$. In such a case, we physically swap the records, update the values of the $S S E$ for both groups, and assign the swapped records at the boundary state. If we failed to find a candidate to be swapped so as to reduce the value of $S S E_{\alpha_{w v}}$, we should not delete this tuple because we are not in a position to confirm the proposition that these 

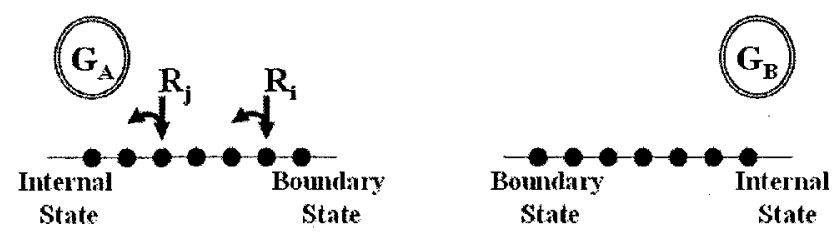

Figure 4.2: The automaton has been rewarded, since both $R_{i}$ and $R_{j}$ are similar and located in the same group.
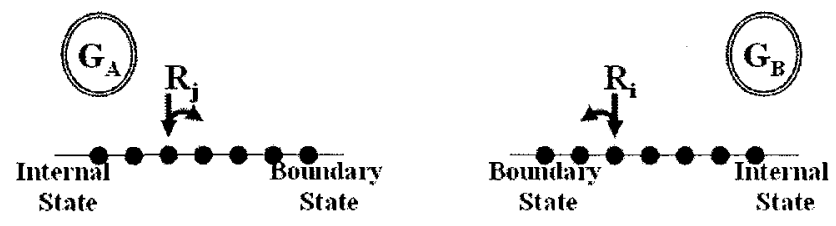

Figure 4.3: The automaton has been penalized, since $R_{i}$ and $R_{j}$ are similar but located in distinct groups. Neither of them is at the boundary state.

two records, being in the same group, will decrease or increase the total $S S E$. This is because both records are in the state of minimum certainty, and, thus, it is likely that during the processing of $\mathbb{L}$ another of the tuples in the similarity list will force one of these records to migrate to another group, rendering the reprocessing of this tuple useful. This scenario is described in Figure 4.5 and Algorithm 10.

For the sake of completeness, (and to not disturb the continuity) the discussed scheme is algorithmically described in Algorithm 6 with the respective modules being given as separate procedures. 


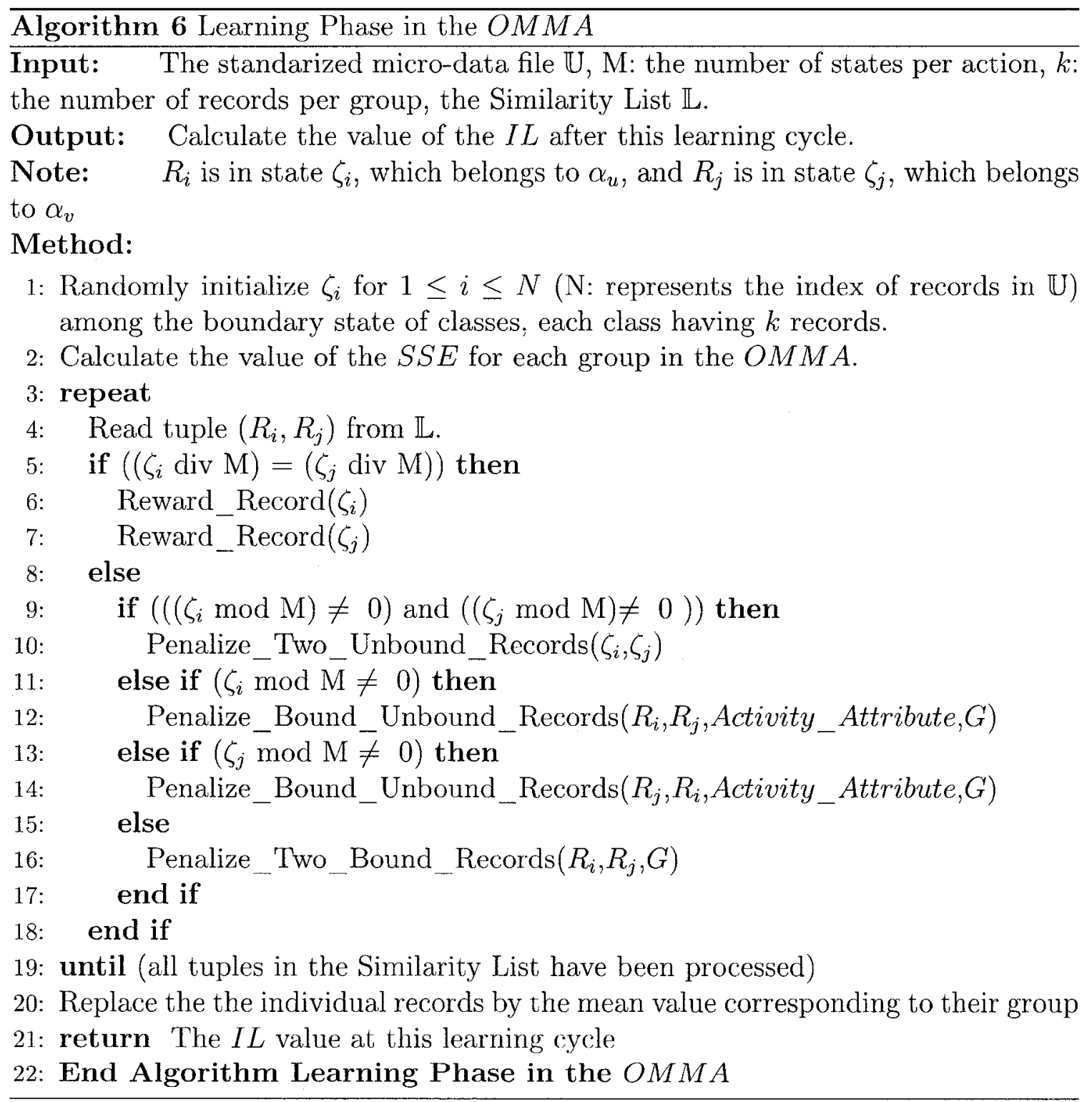



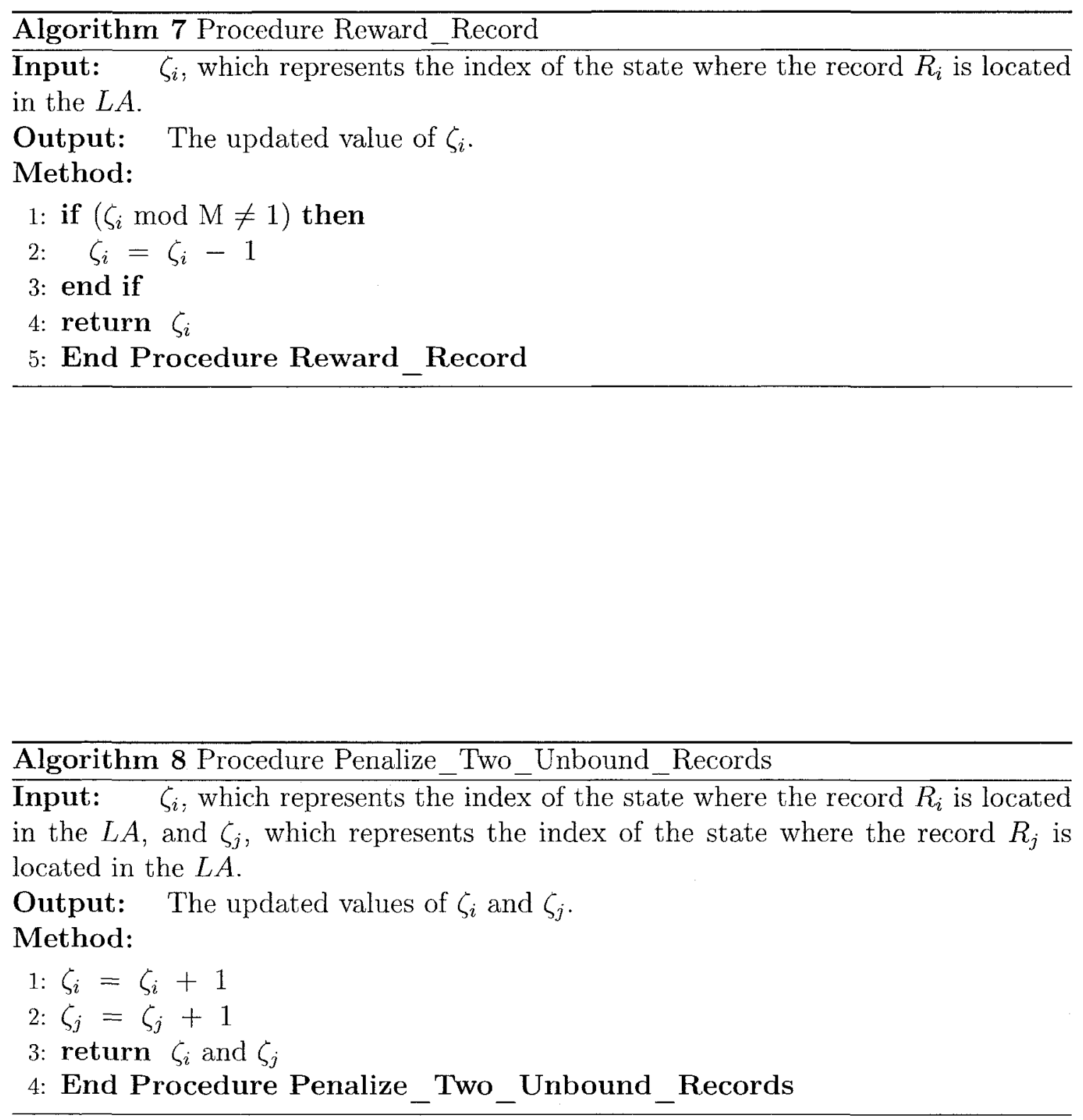


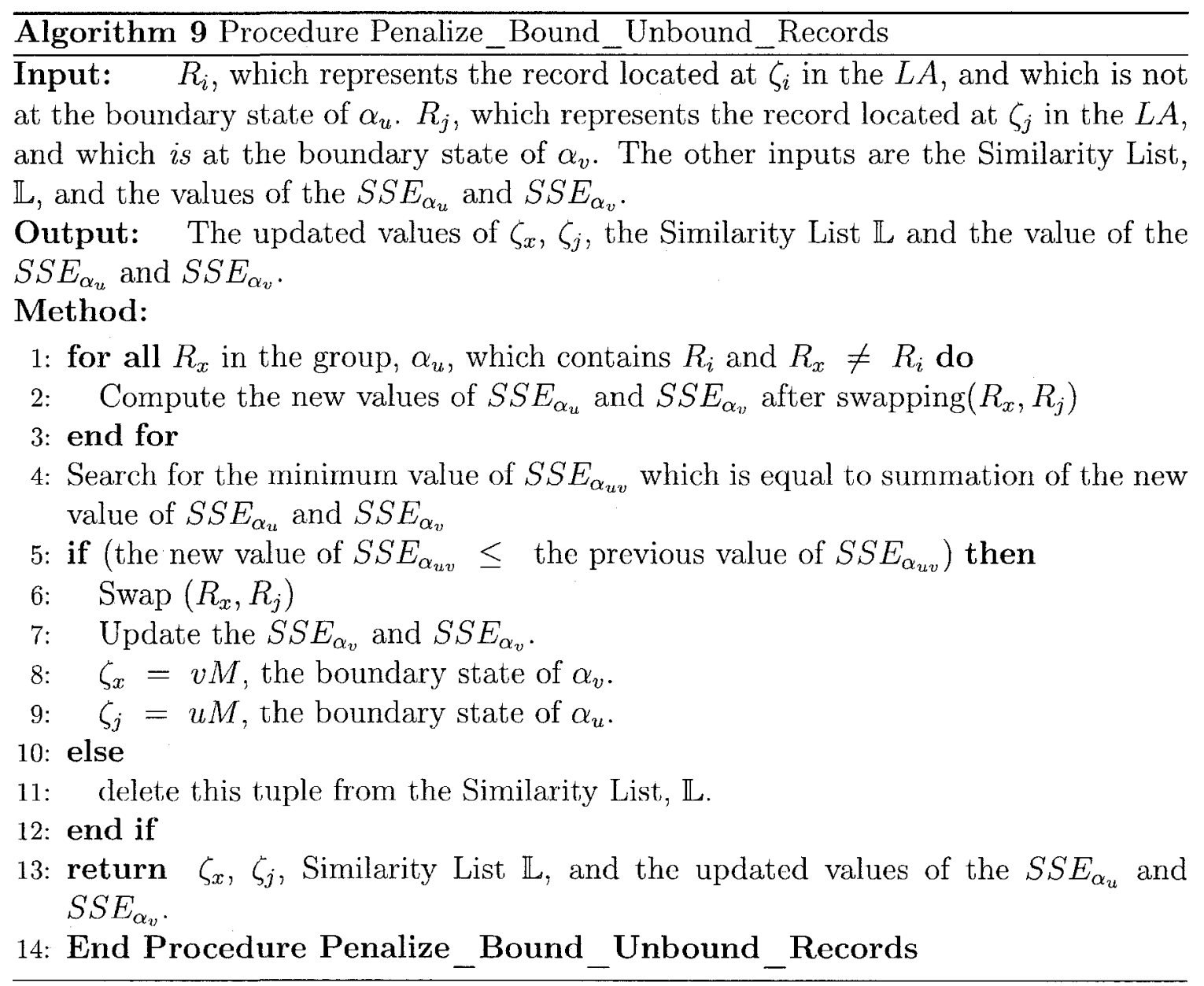




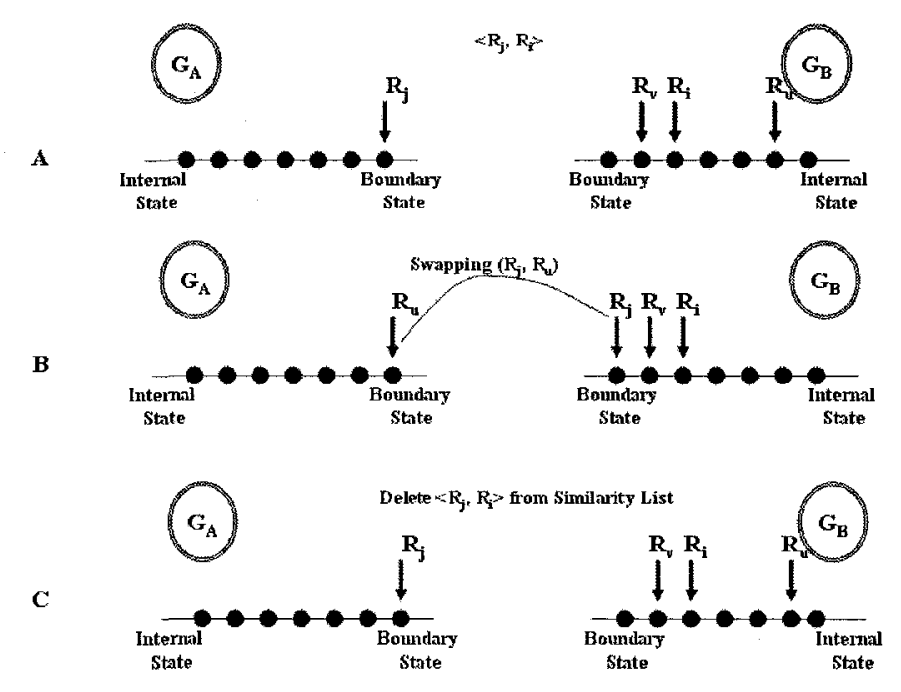

Figure 4.4: (A) The automaton has been penalized, since $R_{i}$ and $R_{j}$ are similar but located in distinct groups. $R_{j}$ is at the boundary state, while $R_{i}$ is not at the boundary state. After searching for the most suitable record that leads to the minimum value of the $S S E$, we have two choices for the scenario when $k=3$. In the first case (see (B) above), we perform a physical swap between $\left(R_{u}, R_{j}\right)$. In the second case, (in (C)), no record-swap leads to a smaller value of the $S S E$, and thus, the tuple $\left\langle R_{i}, R_{j}\right\rangle$ is deleted from the similarity list. 


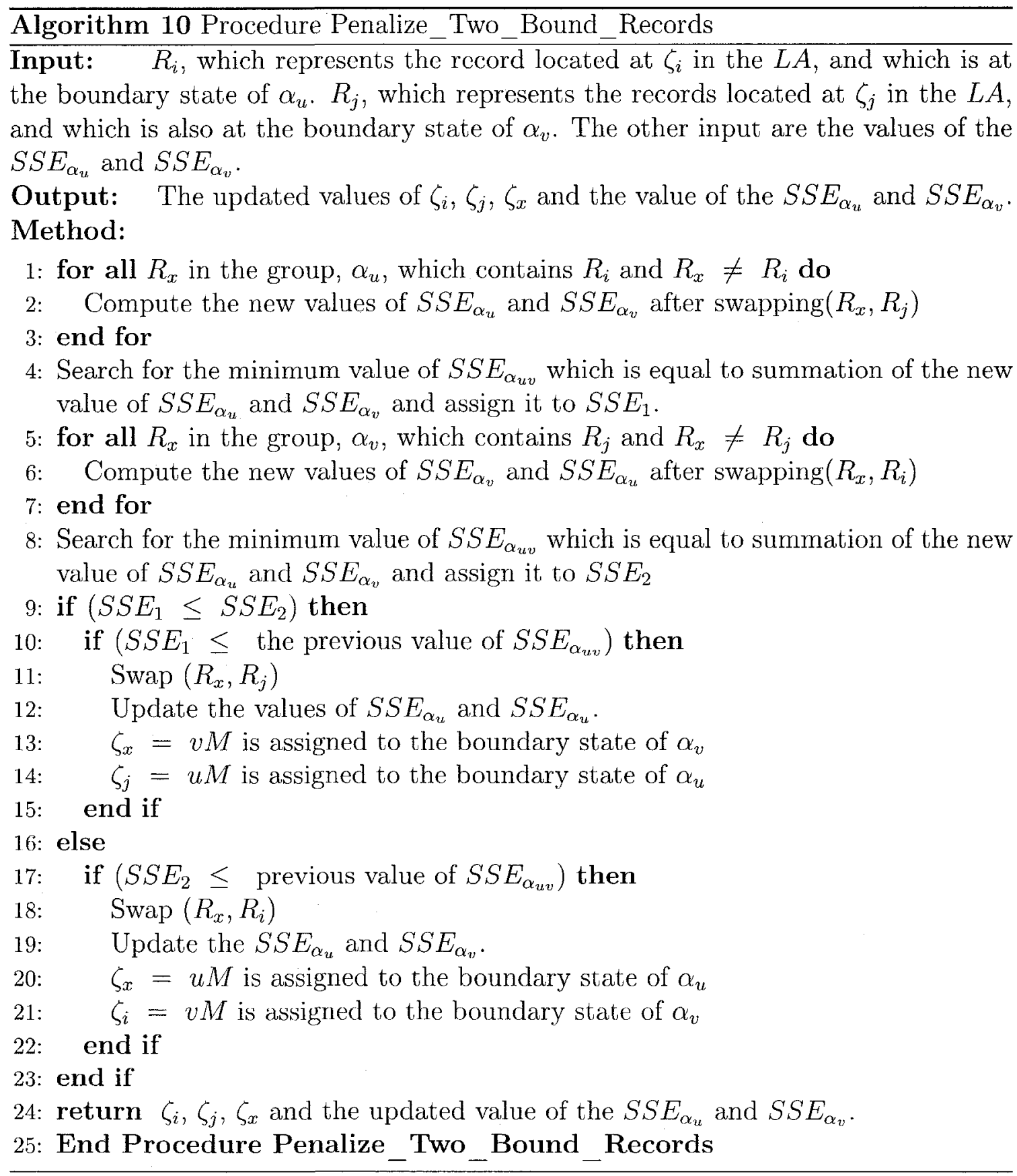




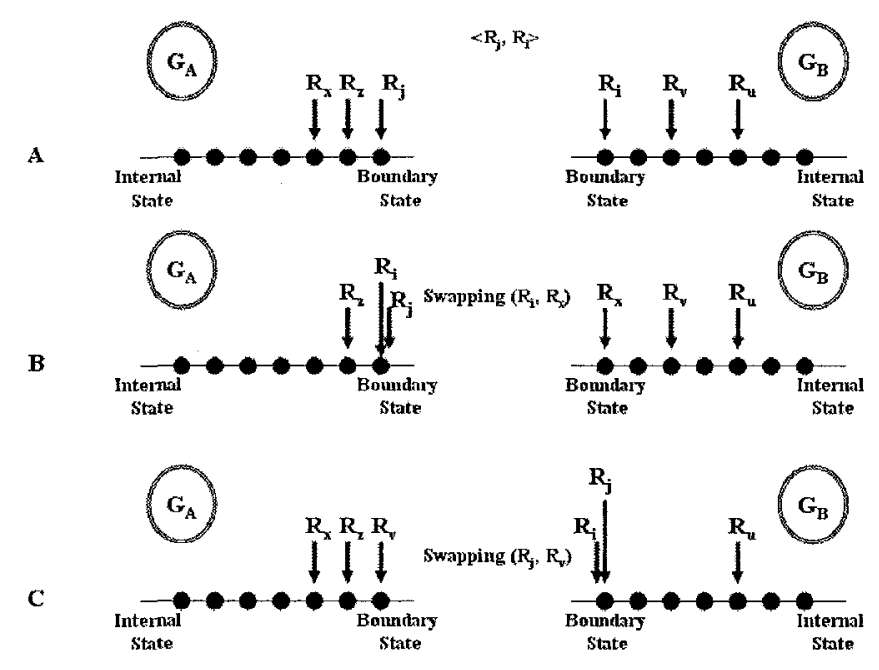

Figure 4.5: (A) The automaton has been penalized, since $R_{i}$ and $R_{j}$ are similar but located in distinct groups. However, both of them are in a boundary state. After searching for the most suitable record that leads to the minimum value of the SSE, we encounter four swapping options for the scenario when $k=3$ - which, in turn, depend on the best candidate record. For example, a physical swap between $\left(R_{i}, R_{x}\right)$ is done when $R_{x}$ is the best candidate (as in case (B)). Similarly, a physical swap between $\left(R_{j}, R_{v}\right)$ is done, when $R_{v}$ is the best candidate, (as in (C)). 


\subsubsection{Formal Properties of The $O M M A$}

We now state and formally prove a property of the $O M M A$.

Theorem 4. The learning process in the OMMA leads to a monotonically decreasing $I L$.

Proof.

We shall prove the theorem by contradiction. To do this we assume that the value of the $I L$ at time $n, I L(n)$, is less than the value of the $I L$ at time $n+1, I L(n+1)$. If the condition $I L(n+1)>I L(n)$ has to be satisfied, it implies that there is at least one movement of two objects (data elements) that causes this increment. This movement could either be a consequence of a reward or a penalty, as seen in Case 1, Case 2, or Case 3 of the $L A$ transition.

Consider the case of a reward. In this case, the pair of objects move one state towards the most internal state. This means that each group continues to have the same objects as in the previous step, which further implies that the value of the $S S E$ of each group does not change. Clearly, such a movement will only result in an identical $I L$.

Consider now the response referred to as Case 1 in the scenario of a penalty. In this case, the respective objects move a single state toward the most external state. Again, each group still has the same objects as in the previous step, which again implies that the value of the $S S E$ of each group does not change. Thus, clearly, an $I L$ - increasing movement could not have occurred.

Consider, finally, the effect of responding as per Case 2 or Case 3 of a penalty. These are the cases when either of the objects are migrated to another partition. However, by virtue of the pre-checking mechanism (Line 5 of Algorithm 4 and Lines 10 and 17 of Algorithm 5) such an $I L$ - increasing could not have occurred. Note that if the summation of the $S S E$ for the groups after the migration is less than or equal to the summation of the $S S E$ for groups prior to it, the objects will migrate; otherwise no movements is permitted.

Since all the four different types of movements lead to values of the $S S E$ which are 
either equal to or less than the total $S S E$ of the previous step, it is clear that the value of the $I L$ at the end of each step is less than or equal to the corresponding value in the previous step. The results follow.

A straight forward Corollary of this is that the $O M M A$ converges to a local minimum of the $I L$. We conjecture (from our experiment results) that it also attains the global minimum with the $\epsilon$-optimal property, but this is, as yet, unproven.

\subsection{Comparing the $M D A V$ and $O M M A$ Methods}

Quantifying the quality of $M A T s$ is based on two criteria, namely the $I L$ and the $D R$, both of which have been explained in $[53,146]$. In this chapter, we also consider how we can compare MATs using a composite measure involving both the $I L$ and the $D R$.

Information Loss: To evaluate the loss in the data utility caused by an $M A T$ on a continuous micro-data file, we seek a measure which assesses how different the masked file is from the original one. Earlier, the quantity $I L$ was measured in a generic way, by essentially estimating how much the data was "harmed" by using the $M A T[22,45,74,84,92]$. Such a measurement does not demonstrate how the statistics have been structurally modified by virtue of invoking the micro-aggregation process, and how much the modification is.

We believe that any criterion to evaluate the quality of the published data set should not be only based on the proximity between the masked and the original data files. Rather, it should additionally examine whether the published file preserves the natural statistical characteristics of the original file, namely, those which can be evaluated by computing some positional moments such as the means, variances, covariances and correlations. Examinations of this sort will definitely help in building a clear image about the potential of the MATs, and in choosing the technique which has the least impact on the desired statistics. 
As mentioned in $[21,48,94,102,119]$, several measures to quantify the $I L$ have been proposed. Five of these measures will be used in this chapter to construct a comparative benchmark. This will be done by assuming that the original and masked micro-data sets are specified in terms of the $n$ ordered individuals such as $\mathcal{X}=\left\{X_{1}, X_{2}, \ldots, X_{n}\right\}$ and $\mathcal{X}^{\prime}=\left\{X_{1}^{\prime}, X_{2}^{\prime}, \ldots, X_{n}^{\prime}\right\}$, respectively. Observe that each $X_{i}$ is an instantiation of the random vector (of dimension $d$ ) $\underline{X}$, whose mean is $\bar{X}$, and each $X_{i}^{\prime}$ is an instantiation of the random variable $\underline{X^{\prime}}$, whose mean is $\bar{X}^{\prime}$. Thus, each data vector in the original and masked data sets can be represented as $X_{i}=\left[x_{i 1}, x_{i 2}, \ldots, x_{i d}\right]^{T}$ and $X_{i}^{\prime}=\left[x_{i 1}^{\prime}, x_{i 2}^{\prime}, \ldots, x_{i d}^{\prime}\right]^{T}$, respectively, where both $x_{i j}$ and $x_{i j}^{\prime}$ are the values associated with the $j^{\text {th }}$ variable. Symbolically:

$\mathcal{X}$ and $\mathcal{X}^{\prime}$ : Are the original and masked data sets.

$\bar{X}$ and $\bar{X}^{\prime}$ : Are the mean vectors of $\underline{X}$ and $\underline{X^{\prime}}$, respectively, where those are computed as $\bar{X}=\frac{1}{n} \sum_{i=1}^{n} X_{i}$ and $\bar{X}^{\prime}=\frac{1}{n} \sum_{i=1}^{n} X_{i}^{\prime}$.

$\mathcal{V}$ and $\mathcal{V}^{\prime}$ : Are the covariance matrices of $\underline{X}$ and $\underline{X^{\prime}}$, respectively. Thus, $\mathcal{V}=\mathrm{E}\left[(\underline{X}-\bar{X})(\underline{X}-\bar{X})^{T}\right]$ and $\mathcal{V}^{\prime}=\mathrm{E}\left[\left(\underline{X^{\prime}}-\bar{X}^{\prime}\right)\left(\underline{X^{\prime}}-\bar{X}^{\prime}\right)^{T}\right]$.

$S$ and $S^{\prime}$ : Are the vectors representing the variance of the components of $\underline{X}$ and $\underline{X}^{\prime}$ respectively, and are given as $S=\operatorname{Diag}[\mathcal{V}]$, and $S^{\prime}=\operatorname{Diag}\left[\mathcal{V}^{\prime}\right]$.

$\mathcal{R}$ and $\mathcal{R}^{\prime}$ : Are the correlation matrices of $\underline{X}$ and $\underline{X^{\prime}}$ respectively. If $\Gamma$ is the diagonal matrix with the standard deviation of the variables along the main diagonal (and with zero's elsewhere), then, $\mathcal{R}=\Gamma^{-1} \mathcal{V} \Gamma^{-1}$, and $\mathcal{R}^{\prime}=\Gamma^{\prime-1} \mathcal{V}^{\prime} \Gamma^{\prime-1}$.

The difference or the "discrepancy" between two matrices can be measured in at least three ways: Mean square error, Mean absolute error, and Mean variation. In this chapter, we use the mean variation ${ }^{5}$ when it concerns data structures, data means, data variance, and data covariance, while the mean absolute error is used to measure the data correlation difference ${ }^{6}$, as shown in Table 4.1. This leads to five

\footnotetext{
${ }^{5}$ The following rule is applied to all the mean variation formulae. If $x_{i j}=0$ and $x_{i j}^{\prime} \neq 0$, then we divide the difference by $\left|x_{i j}^{\prime}\right|$. If $x_{i j}=x_{i j}^{\prime}=0$, the term is not added to the sum.

${ }^{6}$ The mean variation is commonly used because it is a unit-free measure, since the variation is
} 
distinct metrics $M 1, M 2, M 3, M 4$, and $M 5$, whose significance and explicit forms are tabulated in Table 4.1 .

Finally, the overall $I L$, referred to $\left(G_{I L}\right)$, is defined as follows:

$$
G_{I L}=100 * \frac{M 1+M 2+M 3+M 4+M 5}{5},
$$

where the explicit form for $M 1-M 5$ are given in Table 4.1.

Table 4.1: Information loss measures.

\begin{tabular}{|c|c|c|c|}
\hline Metric & Measurement & $\begin{array}{c}\text { Matrix } \\
\text { discrepancy }\end{array}$ & $\begin{array}{c}\text { Mathematical } \\
\text { expression }\end{array}$ \\
\hline \hline$M 1$ & Mean variation of data & $\mathcal{X}-\mathcal{X}^{\prime}$ & $\frac{\sum_{j=1}^{d} \sum_{i=1}^{n} \frac{\left|x_{i j}-x_{i j}^{\prime}\right|}{\left|x_{i j}\right|}}{n d}$ \\
\hline$M 2$ & Mean variation of data means & $\bar{X}-\bar{X}^{\prime}$ & $\frac{\sum_{j=1}^{d} \frac{\left|x_{j}-\bar{x}_{j}^{\prime}\right|}{\left|\bar{x}_{j}\right|}}{d}$ \\
\hline$M 3$ & Mean variation of data variances & $S-S^{\prime}$ & $\frac{\sum_{j=1}^{d} \frac{\left|v_{j j}-v_{j j}^{\prime}\right|}{d v_{j j} \mid}}{d}$ \\
\hline$M 4$ & Mean variation of data covariates & $\mathcal{V}-\mathcal{V}^{\prime}$ & $\frac{\sum_{j=1}^{d} \sum_{1 \leq i \leq j} \frac{\left|v_{i j}-v_{i j}^{\prime}\right|}{\left|v_{i j}\right|}}{\frac{d(d+1)}{2}}$ \\
\hline$M 5$ & Mean absolute error of data correlations & $\mathcal{R}-\mathcal{R}^{\prime}$ & $\frac{\sum_{j=1}^{d} \frac{\sum_{1 \leq i<j}\left|r_{i j}-r_{i j}^{\prime}\right|}{\frac{d(d-1)}{2}}}{2}$ \\
\hline \hline
\end{tabular}

Disclosure Risk: The effect of applying different $M A T s$ is not, really, limited to the $I L$. We contend that the loss of confidentiality as a result of disseminating the published micro-aggregated file must also be analyzed. This is true because the $D R$ does not only depend on the data, but also on the Intruder who knows something about the population. Therefore, any $D R$ assessment must evaluate the risk of providing additional information which can assist in linking a masked record with the corresponding record in the original data set. In addition, we need to divided by the original variable. However, it is not suitable if the original variable is likely to be zero or close to zero, which is the case for data correlations. For near-zero correlations, even a small change would lead to a significant mean variation, and this would be wrongly interpreted as a large $I L$. On the other hand, since the correlations are already unit-free, the mean absolute error was used as a good option for this quantity. 
evaluate the risk that the values of original records can be accurately estimated from the published masked records $[14,94,142]$.

The first kind of risk is evaluated through a strategy known as the Record Linkage Disclosure technique $(R L D)$. Each record in the masked file is linked to its nearest ${ }^{7}$ record in the original file. The linkage computation proceeds by evaluating the distances between each record in the masked file and all the records in the original file. Thereafter, the "nearest" and "second nearest" records for each record in the masked file are considered. A Match is said to have occurred when the index of the published record is equal to the index of either the nearest or the second nearest record in the original file [23, 44, 48, 53]. The number of Matches yields an estimate of the number of published records whose original identity can be re-identified by the Intruder. $D R$ is defined as the proportion of Matches among the total number of records in the original or published file.

To apply this method, researchers generally assume that an Intruder has an external file containing a subset of the key variables that are common to the published file. The Intruder attempts to match the published file and the external file using the subset of common shared variables so as to discover additional information about the original record. The literature reports several disclosure scenarios depending on the number and the identity of the key variables in the original file - which are also assumed to be known to the Intruder.

The second kind of risk is evaluated by means of a so-called confidential Interval Disclosure $(I D)$ technique. In this case, we assume that the Intruder is not attempting to pair masked records with original records. Rather, the Intruder is only interested in determining an approximate value for the original record. The $I D$ operates as follows: Each variable is independently ranked, and a rank interval is defined in the neighborhood of the value that the variable takes on for a specific record, say $r$. The rank of this value should be less than $P \%$ of the total number of records, and the

\footnotetext{
${ }^{7}$ This distance is typically measured using the Euclidean distance. It is worth mentioning that both files are usually standardized $[23,53]$.
} 
value in the center of the interval should correspond to the value of the variable in record $r[23,44,48]$. For example, let us assume that a variable is sorted, and that $r$ is a value taken by that variable in a certain record. Then, the interval centered around value $r$ consists of values rather than ranks, and so the lower bound of the interval equals the value with $\operatorname{Rank}_{L b}=\operatorname{Rank}(r)-P \%$, and the upper value of the interval equals the value $\operatorname{Rank}_{U b}=\operatorname{Rank}(r)+P \%$. A Match is said to have occurred when the values of all variables in the original record fall in the corresponding calculated intervals ${ }^{8}$.

A confidence index for the Intruder for each interval size is evaluated by using this measure, and a smaller interval implies a lower value for the confidence. Normally, the average confidence is computed by using a certain fixed range of percentages (say, between $1 \%$ and $10 \%$ ) of the records. Obviously, a large value of $P$ implies that less information is revealed, and a large value for $D R$.

The overall global $D R$, given as $G_{D R}$, is defined as the average of the $D R$ as computed by both the above strategies, and has the form:

$$
G_{D R}=\frac{R L D+I D}{2}
$$

Overall Scoring Index: As we know, the indices of the $I L$ and $D R$ reflect completely different aspects of an $M A T$. Most researchers have concentrated on either of these criteria. While this is an accepted practice, we feel that a more fair index would be one which considers both of them simultaneously. In this vein, we define the Scoring Index, $S I$, to be a linear combination of the indices obtained for the $I L$ and $D R$ as:

$$
S I=x G_{I L}+(1-x) G_{D R} .
$$

\footnotetext{
${ }^{8}$ At the extreme points, the bounds of the interval are computed as follows: For $r_{m i n}$, the value of $R a n k_{L b}$ is set at $r_{m i n}$, and $R a n k_{U b}$ is assigned the value $\operatorname{Rank}\left(r_{\min }\right)+P \%$. Analogously, for $r_{\max }$, the value of $R a n k_{L b}$ is assigned at the value with $\operatorname{Rank}\left(r_{\max }\right)-P \%$, and $\operatorname{Rank}_{U b}$ is set at $r_{\max }$.
} 
Through out our studies, we have set the value of $x$ to be 0.5 , implying that we give equal weight to the $I L$ and $D R$ measures.

\subsection{Experimental Results}

\subsubsection{Data Sets}

The $O M M A$ has been rigorously tested and the results obtained seem to be very promising. We have tested it using the two real-life benchmark reference data sets used in previous studies and various simulated data sets ${ }^{9}$ using Matlab's built-infunctions:

1. The Tarragona data set which contains 834 records with 13 variables [45].

2. The Census data set which contains 1080 records with 13 variables $|53|$.

3. A uniform distribution $(\min =0 ; \max =1000)$.

4. A normal distribution $(\mu=0 ; \sigma=0.05)$.

5. A uniform distribution $(\min =0 ; \max =40,000)$ which contains 10,000 records and 16 dimensions.

6. A normal distribution $(\mu=500 ; \sigma=150)$ which contains 6,000 records and 16 dimensions.

In all the data sets, discrete variables were approximated as being continuous.

The data sets in items 3 and 4 above involved vectors with dimensions ranging from 12 up to 22 , and sets of cardinality from 1,200 up to 5,400 .

\footnotetext{
${ }^{9}$ The experiments were also done to investigate the scalability of the $O M M A$ with respect to the size of the data, its dimensionality, and the number of records per group.
} 
It is worth mentioning that the generated simulated data sets have been generated to mimic real-life scenarios [102]. To achive this, the resulting simulated data sets, described in items 5 and 6 above, had two properties that were important to our experiments:

1. Key variables are necessary to estimate the disclosure risk using $R L D$. Therefore, the selection criterion of the key variables was based on choosing the minimum number of repetitions of values in each variable. More specifically, 3 key variables were chosen for the uniform data sets, while 5 key variables were chosen for the normal data set.

2. The number of records in each data set is based on the number of key variables. In general, the size of the simulated data should be low, as one would not expect repeated values for continuous variables. However, there were repetition in the data set. Our selection of 4,800 records in the uniform data, and 1,560 in the normal data set, because this was the cardinality of the set which corresponded to the largest integer which is a multiple of $3,4,5$ and 6 . Thus, the $M A T$ could be invoked with a minimum group size of $k=3,4,5$ or 6 .

\subsubsection{Results}

For a given value of the minimum group size $k$, we compared the percentage value of the $I L=S S E / S S T$ resulting from the $O M M A$ and the $M D A V$ strategies. It is important to mention that the $M D A V$ was implemented based on the centroid concept and not a diameter concept ${ }^{10}$. The memory value, $M$, was set to be 5 for all the experiments, since it yielded the best solution and a reasonable computational time. All the programs were written in the $C^{++}$language, and the tests were performed on an Intel(R) Pentium (R)M Processor with the clock speed of $1.73 \mathrm{GHz}$, and with $512 M B$ of $R A M$. The $O M M A$ was applied to the uni-variate data sets and the

\footnotetext{
${ }^{10}$ We did not program the $M D A V$ scheme. We are extremely thankful to Dr. Francesc Sebe for giving us his source code.
} 
multi-variate data sets.

Table 4.2: Comparison of the percentage of the $I L$ between the $M D A V$ and the $O M M A$ on the Tarragona and Census data sets (uni-variate Methods). In this case the value of $k$ was set to be $k=3$. The column "Converge" indicates the index of the cycle in the learning phase when the value of the total SSE does not change.

\begin{tabular}{|c|c|c|c|c|c|c|c|}
\hline \multicolumn{3}{|c|}{ Tarragona Data Set } & \multicolumn{3}{c|}{ Census Data Set } \\
\cline { 1 - 4 } Variable & $M D A V$ & \multicolumn{2}{|c|}{$O M M A$} & \multirow{2}{*}{ Variable } & $M D A V$ & \multicolumn{2}{c|}{$O M M A$} \\
\cline { 2 - 3 } & IL & IL & Converge & & IL & L & Converge \\
\hline \hline Var1 & 7.15200 & 7.15199 & 2 & Var1 & 0.13155 & 0.13155 & 4 \\
\hline Var2 & 0.63586 & 0.63586 & 4 & Var2 & 0.00138 & 0.00138 & 4 \\
\hline Var3 & 0.51702 & 0.51702 & 5 & Var3 & 0.00828 & 0.00828 & 5 \\
\hline Var4 & 1.48854 & 1.48854 & 4 & Var4 & 0.00489 & 0.00489 & 4 \\
\hline Var5 & 1.69394 & 1.69394 & 5 & Var5 & 0.02449 & 0.02449 & 4 \\
\hline Var6 & 0.47503 & 0.47503 & 4 & Var6 & 0.03262 & 0.03262 & 4 \\
\hline Var7 & 1.96623 & 1.96623 & 5 & Var7 & 0.00171 & 0.00172 & 4 \\
\hline Var8 & 0.42182 & 0.42182 & 4 & Var8 & 0.43418 & 0.43418 & 5 \\
\hline Var9 & 1.28625 & 1.28627 & 4 & Var9 & 0.72176 & 0.72176 & 5 \\
\hline Var10 & 1.74929 & 1.74929 & 4 & Var10 & 0.00611 & 0.00611 & 5 \\
\hline Var11 & 2.58368 & 2.58367 & 3 & Var11 & 0.01353 & 0.01353 & 4 \\
\hline Var12 & 4.14703 & 4.14703 & 5 & Var12 & 0.00689 & 0.00689 & 5 \\
\hline Var13 & 5.00563 & 5.00563 & 4 & Var13 & 0.00808 & 0.00808 & 8 \\
\hline \hline
\end{tabular}

Table 4.2 shows a comparison between the $M D A V$ and the $O M M A$. In this table, both strategies have been applied on uni-variate data sets, for each of the "Tarragona" and "Census" data sets containing 13 continuous variables, and the value of $k$ was set to 3 in all the experiments. The results clearly show that the $O M M A$ obtains values of the $I L$ exactly same as those obtained by the $M D A V$ scheme. The reason for this appears to be the uniqueness of the optimal solution in the uni-variate case when $n$ is multiple of $k$. It is worth mentioning, that the $O M M A$ reaches the minimum value of the $I L$ with an average of 4 successive learning cycles in the Tarragona data set, while 5 cycles are required, on the average, to reach the minimum value of the $I L$ in the Census data set. The computation time required to micro-aggregate each variable independently was as low as 1.93 seconds (on the average) for the Tarragona data set, while for the Census data set it was, on the average, about 2.93 seconds. We also can unequivocally conclude that for uni-variate micro-aggregation the time required 
to micro-aggregate all the individual records to reach the minimum $I L$ increases with respect to the data size. Besides, the number of learning cycles, required to lead to the minimum value of the $I L$, is proportional to the computation time.

Table 4.3: Comparison of the percentage of the $I L$ and the computation time between the $M D A V$ and the $O M M A$ on the real-life data sets (Tarragona and Census), and simulated data sets (Uniform and Normal distributions) for multi-variate methods.

\begin{tabular}{|c|c|c|c|c|c|c|c|}
\hline $\begin{array}{c}\text { Data } \\
\text { Set }\end{array}$ & $\mathrm{k}$ & \multicolumn{2}{|c|}{$M D A V$} & \multicolumn{3}{|c|}{ OMMA } & Improv. \\
\cline { 3 - 7 } & value & $\mathrm{IL}$ & Time & IL & Time & Converge & $(\%)$ \\
\hline \hline \multirow{3}{*}{ Tarragona } & 2 & 9.2750 & 0.28 & 9.2435 & 5.44 & 7 & $0.34 \%$ \\
& 3 & 16.9661 & 0.20 & 15.1290 & 11.69 & 5 & $10.83 \%$ \\
& 6 & 26.4047 & 0.11 & 24.2609 & 36.08 & 5 & $8.12 \%$ \\
\hline \hline \multirow{3}{*}{ Census } & 3 & 5.6535 & 0.33 & 5.2290 & 20.16 & 5 & $7.51 \%$ \\
& 4 & 7.4414 & 0.25 & 6.7623 & 30.00 & 4 & $9.13 \%$ \\
& 5 & 8.8840 & 0.27 & 8.0900 & 48.84 & 4 & $8.94 \%$ \\
& 6 & 10.1941 & 0.24 & 9.1429 & 63.97 & 4 & $10.31 \%$ \\
\hline \hline \multirow{3}{*}{ Uniform } & 3 & 22.4608 & 4.18 & 21.3214 & 33.50 & 7 & $5.34 \%$ \\
distribution & 4 & 29.1714 & 4.45 & 26.2564 & 60.21 & 7 & $11.10 \%$ \\
& 5 & 33.9636 & 4.62 & 29.8415 & 70.11 & 7 & $13.81 \%$ \\
\hline \hline \multirow{2}{*}{ Normal } & 6 & 37.1577 & 4.75 & 32.8529 & 125.59 & 7 & $13.10 \%$ \\
\hline distribution & 3 & 26.9348 & 0.45 & 25.1386 & 1.15 & 6 & $7.15 \%$ \\
& 5 & 33.9256 & 0.78 & 30.7705 & 1.80 & 6 & $10.25 \%$ \\
& 6 & 43.4592 & 0.59 & 35.1803 & 2.03 & 6 & $12.46 \%$ \\
\hline \hline
\end{tabular}

The power of the OMMA is clearly evident when it is used on multi-variate data sets, since the $O M M A$ has the ability to measure the similarity between the individual records based on two different criteria. The first criterion, $C_{1}$, involves studying the relation between the records quantified in terms of the distance between each record and the other records. As opposed to this, the second criterion, $C_{2}$, attempts to maintain the $S S E$ as low as possible. The $O M M A$ has the ability to prioritize these two criteria, and to reflect the corresponding evaluation in the process of awarding rewards and penalties. In the case of a reward, the potential of having any two records in the same group is increased by moving both of them, one state towards the most internal states. Thus, in this setting, $C_{1}$ is given a higher priority than $C_{2}$. Similarly, in the scenario of a penalty (see Case 1), processing the tuple 
and moving both records one state towards the boundary state having the minimum certainty (while preserving the minimum value of $S S E$ ), again lends $C_{1}$ a higher priority than $C_{2}$. But, in Case 2 and Case 3 when a penalty response is processed, the $O M M A$ forces the corresponding records to migrate to groups that preserve the number of records in each group, and to move towards the condition in which the absolute minimum value of the $S S E$ is obtained. This has the effect of giving $C_{2}$ a higher priority than $C_{1}$.

Table 4.3 shows the results of using the $O M M A$ on multi-variate data sets, where we micro-aggregate all the individual records with all the variables simultaneously. In this case, we have tested the $O M M A$ with different values of $k$ in order to determine the effect of increasing the number of records per group. It seems to be apparent that increasing the number of records per group tends to increase the value of the $I L$ besides increasing the required computational time.

Table 4.3 shows that the values of the $I L$ measured for the $O M M A$ were less than the corresponding values measured for the $M D A V$. The percentage of improvement in the $I L$ was as high as $10.83 \%$ in the Tarragona data set when $k=3$, requiring only 11.68 seconds. Similarly, in the Census data set the improvement was as high as $10.31 \%$ when $k=6$, requiring 63.97 seconds. But in case of simulated data sets, the improvement in the $I L$ reached up to $13.81 \%$ when $k=5$ in the uniform data set, and it was as high as $12.84 \%$ when $k=6$ in the normal data set. In term of comparison, we believe that minimizing the loss in the data utility is more important than minimizing the computational time, especially because micro-aggregation is usually performed off-line where additional time resources are less stringent. However, the question of how the decrease in $I L$ is related to the increase in computation time is still open.

To be consistent with the state-of-the-art, we have also compared our IL values with the corresponding loss values yielded by the $M S T$ in [84]. In the Tarragona data set, the percentage improvement in the $I L$ was up to $3.02 \%$ when $k=3$. Similarly, in the Census data set, the percentage improvement in the $I L$ reached up to $3.52 \%$ when $k=3,6.34 \%$ when $k=4$, and $8.17 \%$ when $k=5$. This clearly demonstrates that 
the $O M M A$ competes in a superior manner against all the state-of-the-art strategies. The authors of [84] had suggested that a MST partitioning-based method should be considered as a potential candidate for any practical application. In this context, we recommend using the $O M M A$ in micro-aggregating a multi-variate data set.

Table 4.4: Scoring the $M D A V$ and $O M M A$ with respect to the $G_{I L}$ and $G_{D R}$ by computing the index $S I$, for $k=3,4$, and 5 by using Census data set and the simulated Uniform and Normal distributed data sets.

\begin{tabular}{|c|c|c|c|c|c|c|c|}
\hline Data & Creterion & \multicolumn{2}{|c|}{$k=3$} & \multicolumn{2}{c|}{$k=4$} & \multicolumn{2}{c|}{$k=5$} \\
\cline { 2 - 8 } & & $M D A V$ & $O M M A$ & $M D A V$ & OMMA & $M D A V$ & $O M M A$ \\
\hline \hline \multirow{4}{*}{ Census } & $G_{I L}$ & 28.61000 & 22.81600 & 33.25400 & 28.40800 & 38.67200 & 38.62400 \\
\cline { 2 - 8 } & $R L D$ & 60.71330 & 63.14640 & 49.70570 & 53.53510 & 42.09810 & 48.58860 \\
\cline { 2 - 8 } & $I D$ & 1.98148 & 2.25926 & 0.73148 & 1.02778 & 0.26852 & 0.61111 \\
\cline { 2 - 8 } & $G_{D R}$ & 31.34739 & 32.70283 & 25.21859 & 27.28144 & 21.18331 & 24.59986 \\
\cline { 2 - 8 } & $S I$ & 33.80500 & 30.90800 & 36.12700 & 33.70400 & 38.83600 & 38.81200 \\
\hline \hline \multirow{5}{*}{ Uniform } & $G_{I L}$ & 115.43193 & 41.28212 & 116.11979 & 45.16758 & 120.91989 & 56.38516 \\
\cline { 2 - 8 } & $R L D$ & 0.10880 & 1.09259 & 0.06944 & 0.86343 & 0.06019 & 0.55093 \\
\cline { 2 - 8 } & $I D$ & 0.00417 & 4.33611 & 0.00000 & 1.25625 & 0.00000 & 0.50069 \\
\cline { 2 - 8 } & $G_{D R}$ & 0.05648 & 2.71435 & 0.03472 & 1.05984 & 0.03009 & 0.52581 \\
\hline \hline \multirow{5}{*}{ Normal } & $S I$ & 57.74420 & 21.99824 & 58.07725 & 23.11371 & 60.47499 & 28.45549 \\
\cline { 2 - 8 } & $G_{I L}$ & 108.98124 & 76.07109 & 89.03977 & 40.14807 & 110.86944 & 51.84437 \\
\cline { 2 - 4 } & 0.64936 & 10.74100 & 0.65705 & 7.34936 & 0.2814 & 5.40449 \\
\cline { 2 - 8 } & $G_{D R}$ & 0.00855 & 1.57692 & 0.00000 & 0.39103 & 0.00000 & 0.09829 \\
\cline { 2 - 8 } & $S L$ & 54.65510 & 41.11503 & 44.68415 & 22.00913 & 55.50507 & 27.29788 \\
\hline \hline
\end{tabular}

The other set of experiments were carried to test the SI of $M D A V$ and $O M M A$. Therefore, they have been scored as per the $S I$ index on the simulated data sets and the Census ${ }^{11}$ data set, which contains seven key variables: $\operatorname{Var} 1, \operatorname{Var} 2, \operatorname{Var} 3, \operatorname{Var} 5$, $\operatorname{Var} 10, \operatorname{Var} 11$, and $\operatorname{Var} 12$.

Table 5.2 displays the $S I$ for the $M D A V$ and $O M M A$ methods for various values of $k$, which was set to be either 3,4 or 5 as per the accepted requirements stated in $[46,115,140]$. As per Equation (4.2), the $G_{I L}$ was computed by averaging the values of $M_{1}, M_{2}, M_{3}, M_{4}$, and $M_{5}$. In general, the value of the $G_{I L}$ is directly proportional

\footnotetext{
${ }^{11}$ Scoring them against the Tarragona data set is meaningless because the latter contains no socalled key variables.
} 
to the number of records per group represented by the value, $k$. Therefore, in the Census data set, the best value of $G_{I L}$ for the $O M M A$ was obtained when $k=3$, and was equal to $22.816 \%$, while the best value of $G_{I L}$ for the $M D A V$ was $28.610 \%$ when $k=3$. In the context of the simulated data sets, and in general, the value of $G_{I L}$ for the $O M M A$ is almost half the value that was obtained by using the $M D A V$ (i.e., the value of $G_{I L}$ for the $O M M A$ in the uniform data set and when $k=3$ was $41.28 \%$, while it was equal to $115.43 \%$ for the $M D A V)$. Clearly, the $O M M A$ method preserves the data utility more efficiently than the state-of-the-art, $M D A V$.

We also compared the $G_{D R}$ (which estimates the risk of data being disclosed) using the $R L D$ and $I D$ techniques. The $R L D$ was computed using distance-based metric computations, where the average value was computed over several scenarios. Each scenario computed the average of the $R L D$ over all possible $\left(\begin{array}{l}S \\ C\end{array}\right)=\frac{S !}{C !(S-C) !}$ combinations, where $S$ is the number of key variables in the micro-data file, and $C$ is the number of chosen variables that are known to the Intruder. The results (Table 5.2) generally shows that estimating the risk of disclosing the secure information using the $R L D$ method is inversely proportional to the number of records per group represented by $k$. Thus, in the Census data set, the percentage value of the $R L D$ for the $M D A V$ was as low as $60.71 \%$ when $k=3,49.70 \%$ when $k=4$, and $42.10 \%$ when $k=5$, while it was $63.15 \%$ when $k=3,53.54 \%$ when $k=4$, and $48.59 \%$ when $k=5$ for the $O M M A$. Similarly, simulated data sets report the same fact which is that the $M D A V$ method is more secure than the OMMA method in estimating the $R L D$, for example, the percentage value of the $R L D$ by using the $M D A V$ on the uniform data set was around $0.1 \%$, while it was around $1.0 \%$ by using the OMMA. On the other hand, the percentage value of the $I D$ was calculated as the average value for various settings of $P,(1,2, \ldots, 10 \%)$ on Census data set, when the value of $k$ was assigned to be 3,4 , and 5 . We observe that the minimum percentage value of the $I D$ was equal to $0.26852 \%$, which was achieved by invoking the $M D A V$ when $k=5$. As opposed to this, the minimum percentage value of the $I D$ for the $O M M A$ was almost comparable - to $0.6111 \%$ when $k=5$. In the simulated data sets, the percentage value of the $I D$ was equal to zero for the $O M M A$ and $M D A V$. Therefor, the range 


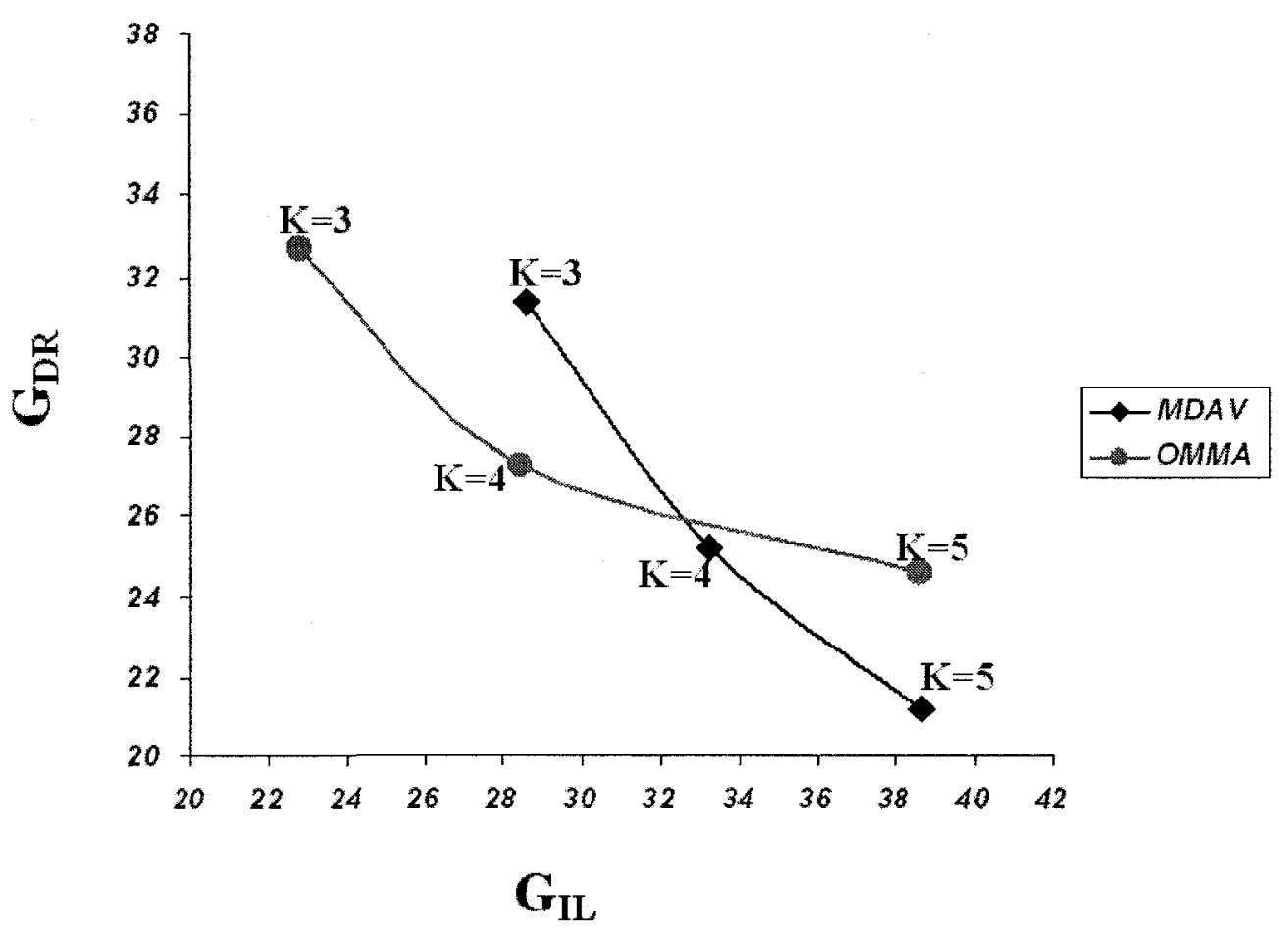

Figure 4.6: The effect of invoking the $M D A V$ and $O M M A$ on the $G_{I L}$ and $G_{D R}$ indices when $k=3,4$ and 5 for Census data set.

of the $p$ values was extended to reach up to $30 \%$ of the original size of the data sets. The percentage value of the $M D A V$ remained zero, while the minimum percentage value for the $O M M A$ reached up to $0.5 \%$ and $0.098 \%$ when $k=5$ in the uniform and normal data sets, respectively.

Finally, the $S I$ value was computed for each $M A T$ based on Equation (4.4). Observe that a lower score value implies a superior performance. From the table we see that the $O M M A$ technique has, almost consistently, a better performance index than the $M D A V$ not only from the $G_{I L}$ perspective, but also as from the perspective of a combination of the $G_{I L}$ and $G_{D R}$ for different values of the $k$. Thus, for example, in Census data set the $O M M A$ method scores the minimum value that was equal to 30.91 when $k=3$, and to 33.704 when $k=4$. Besides the $O M M A$ scores almost half 
the $S I$ value which was obtained by the $M D A V$ by using the simulated uniform and normal data sets.

An interesting exercise involves studying how the $O M M A$ and the $M D A V$ compared when "all" the factors coming to play. In other words, it would be good if we could understand the conditions when one algorithm is superior to the other and vice verse. While this can be done in many ways, we have plotted the $G_{I L}$ versus the $G_{D R}$ for the Census data set, in Figure 5.2, for both schemes. This figure presents sets of paired values of $G_{I L}$ and $G_{D R}$ for the respective algorithm for different values of $k$ ranging from 3 to 5 . The user should observe the influence of a masked method as the value of $k$ changes. From the curve we see that the OMMA optimizes these conflicting criteria in a superior way than the stat-of-the-art $M A D V$ method, because as the value of $k$ increases, the increase of $G_{I L}$ does not effect the value of $G_{D R}$ in the OMMA method as it does in the case of the for $M D A V$.

Finally, we discuss the performance of the $O M M A$ and $M D A V$ with respect to three other issues.

\section{The scalability of the $O M M A$ with respect to cardinality.}

We have tested both the schemes on the uniform and the normal distributions with data set sizes cardinalities $1200,2400,3000,4500$, and 5400, with 16 variables and $k=3$, as shown in Table 4.5. The impact of increasing the size of the data set leads to minimizing the $I L$ value, increasing the computational time and, in the case of the $O M M A$, increasing the number of learning cycles required to reach to the minimum value of the $I L$. The percentage of the improvement in the $I L$ that the OMMA obtains, ranges from $6.69 \%$ to $3.03 \%$ when the data size equals 1,200 and 4,500, respectively, for the uniform distribution, and for the normal distribution, this ranges from $5.46 \%$ when the data size equals 1,200 to $3.03 \%$ when the data size is 4,500 . As the reader can observe, Figures 4.7 and 4.8 display similar trends for minimizing the value of the $I L$ as a function of the data set size for uniform and normal distributions, respectively. 


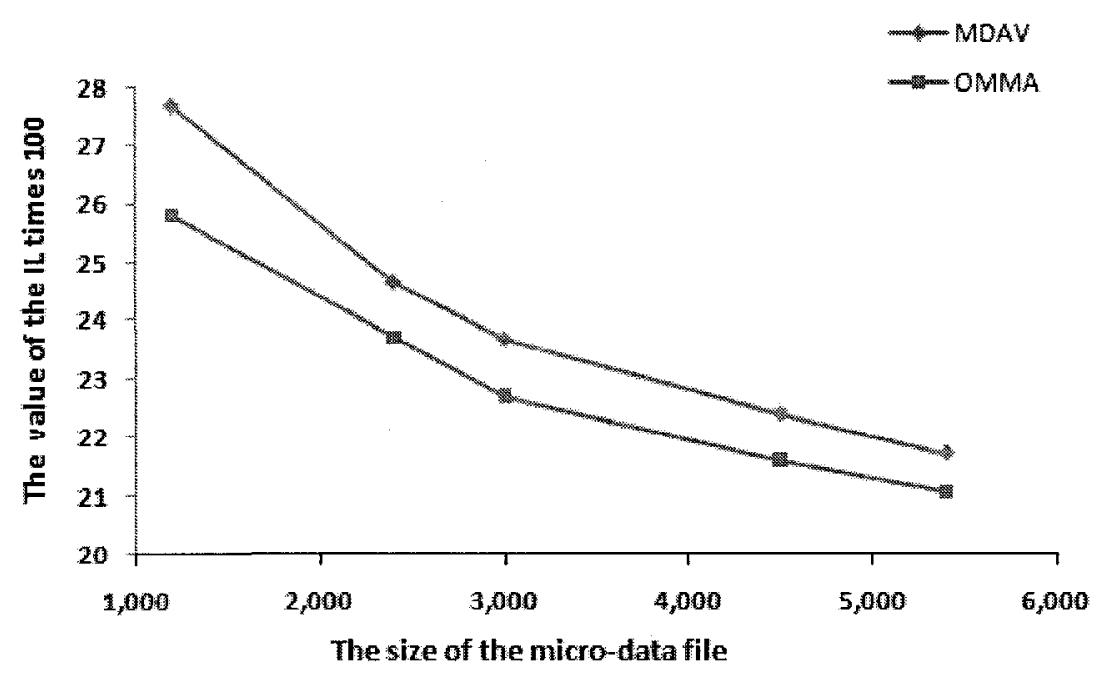

Figure 4.7: The improvement of the $O M M A$ in reducing the percentage value of the $I L$ as a function of the cardinality of the data set using uniformly distributed data when $k=3$.

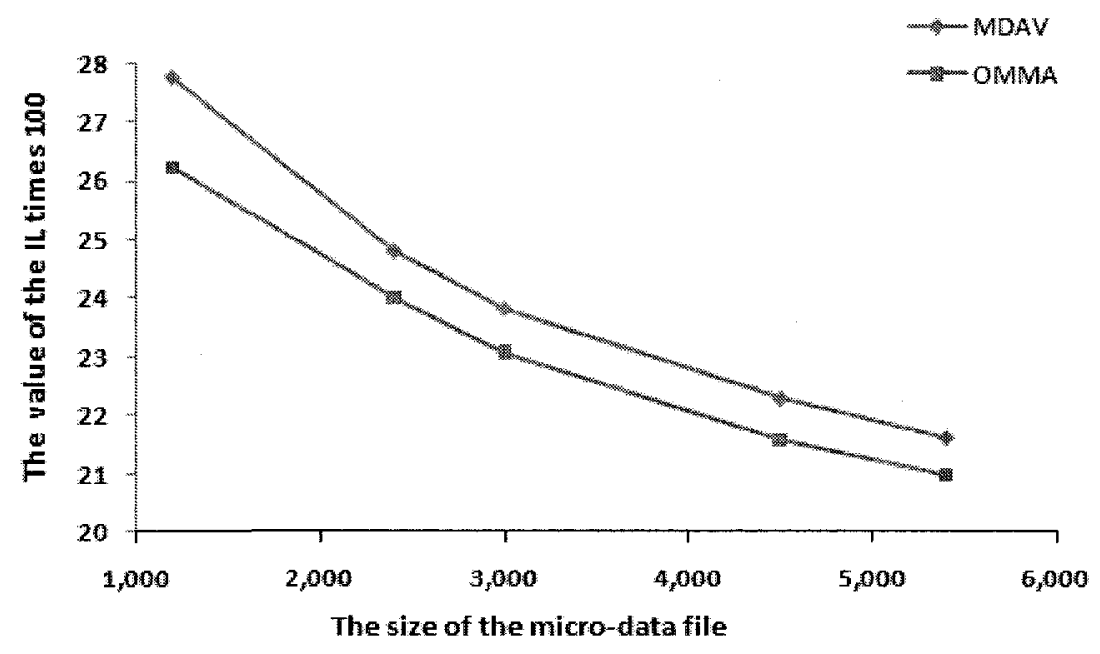

Figure 4.8: The improvement of the $O M M A$ in reducing the percentage value of the $I L$ as a function of the cardinality of the data set using normally distributed data when $k=3$. 
Table 4.5: Comparison of the percentage of the $I L$ and the computation time between the $M D A V$ and the $O M M A$ on simulated uniform and normal data sets for multivariate methods. This table investigates the scalability with respect to data size.

\begin{tabular}{|c|c|c|c|c|c|c|c|c|c|c|}
\hline \multirow{2}{*}{$\begin{array}{l}\text { Data } \\
\text { Set }\end{array}$} & \multirow{2}{*}{$\begin{array}{l}\text { No. of } \\
\text { Records }\end{array}$} & \multirow{2}{*}{$\begin{array}{l}\text { No. of } \\
\text { Groups }\end{array}$} & \multirow{2}{*}{ Dim. } & \multirow{2}{*}{$\begin{array}{c}\mathrm{k} \\
\text { value }\end{array}$} & \multicolumn{2}{|c|}{$M D A V$} & \multicolumn{3}{|c|}{$O M M A$} & \multirow{2}{*}{$\begin{array}{c}\text { Improv. } \\
(\%)\end{array}$} \\
\hline & & & & & IL & Time & IL & Time & onverge & \\
\hline \multirow{5}{*}{$\begin{array}{l}\text { Uniform } \\
\text { Distribution }\end{array}$} & 1,200 & $\overline{400}$ & \multirow{5}{*}{16} & \multirow{5}{*}{3} & 27.67671 & 0.42 & 25.82418 & 0.66 & 6 & 6.69 \\
\hline & 2 , & 800 & & & $24.64686 \mid$ & 1.28 & 23.71665 & 5.13 & 6 & 3.77 \\
\hline & 3,000 & 1000 & & & $23.66435 \mid$ & 2.00 & 22.69465 & 9.88 & 8 & 4.10 \\
\hline & 4,500 & 1500 & & & $22.38678 \mid$ & 4.45 & 21.60147 & 21.64 & 7 & 3.51 \\
\hline & 5,400 & 1800 & & & 21.71397 & 6.42 & 21.05564 & 33.05 & 7 & 3.03 \\
\hline \multirow{5}{*}{$\begin{array}{c}\text { Normal } \\
\text { Distribution }\end{array}$} & 1,200 & 40 & \multirow{5}{*}{16} & \multirow{5}{*}{3} & 1187 & 0.36 & 26.22697 & 0.65 & 7 & $\overline{5.46}$ \\
\hline & & 81 & & & 24.80187 & 1.28 & 24.00719 & 3.76 & 6 & 3.20 \\
\hline & 3,000 & 1000 & & & 23.82211 & 1.99 & 23.06686 & 6.45 & 7 & 3.17 \\
\hline & 4,500 & 1500 & & & 22.29773 & 4.45 & 21.57716 & 16.23 & 6 & 3.23 \\
\hline & 5,400 & 1800 & & & 21.62495 & 6.46 & 20.96986 & 29.97 & 9 & 3.03 \\
\hline
\end{tabular}

\section{The scalability of the $O M M A$ with respect to dimensionality.}

We have also tested both strategies on the uniform and the normal distributions for different numbers of variables, including 12, 14, 16, 18, 20, 22 and 24, when the data size was set to 3,000 and the value of $k$ was set to 3 , as shown in Table 4.6. Again, we observe that, the value of the $I L$ is proportional to the number of the variables used in the micro-aggregation process. Increasing the dimensionality implies increasing the loss in the utility of the information. This makes sense, because, informally speaking, increasing the dimensionality leads to minimizing the similarity between the individual records and, at the same time, reducing the correlation between the different multi-variate records. The interesting point here is that the computational time required to micro-aggregate all the individual records seems to be inversely proportional to the dimensionality in the OMMA scheme, but proportional for the $M D A V$ scheme. This can be justified as follow: In the $O M M A$ case, increasing the dimensionality seems to minimize the within-group similarity, and simultaneously reduce the correlation between them. This, thus, leads to a smaller size for the similarity list structures, which in turn, obviously, requires less time to process all the tuples. As opposed to this, in the case of the $M D A V$, increasing the dimensionality 
requires more time to achieve the computation of the distances between the multi-variate records. The highest percentage of the improvement in the $I L$ is $4.04 \%$ when the number of variables is 16 for the uniform distribution, while for the normal distribution, the highest percentage advantage is $4.52 \%$ when the number of variable is 12 . It should be mentioned that, the $O M M A$ works perfectly when the number of variables used to micro-aggregate the data set is moderate and not too high. Thus, for the uniform distribution with the dimensionality of 22 , and the normal distribution with a dimensionality of 24 , the $O M M A$ is faster than the $M D A V$, while the value of the $I L$ increases. Figures 4.9 and 4.10 display similar trends for minimizing the value of the $I L$ as a function of the dimension of the data set for uniform and normal distributions, respectively. It is worth mentioning that the value of the $I L$ is proportional to the dimension of the variables used in the micro-aggregation process.

Table 4.6: Comparison of the percentage of the $I L$ and the computation time between the $M D A V$ and the $O M M A$ on simulated uniform and normal data sets for multivariate methods. This table investigates the scalability with respect to the data dimensionality.

\begin{tabular}{|c|c|c|c|c|c|c|c|c|c|c|}
\hline \multirow{2}{*}{$\begin{array}{l}\text { Data } \\
\text { Set }\end{array}$} & \multirow{2}{*}{$\begin{array}{l}\text { No. of } \\
\text { Records }\end{array}$} & \multirow{2}{*}{$\begin{array}{l}\text { No. of } \\
\text { Groups }\end{array}$} & \multirow{2}{*}{ Dim. } & \multirow{2}{*}{\begin{tabular}{|c|}
$\mathrm{k}$ \\
value
\end{tabular}} & \multicolumn{2}{|c|}{$\overline{M D A V}$} & \multicolumn{3}{|c|}{$\overline{O M M A}$} & \multirow{2}{*}{$\begin{array}{c}\text { Improv. } \\
(\%)\end{array}$} \\
\hline & & & & & $\overline{\mathrm{IL}}$ & Time & IL & Time & Converge & \\
\hline \multirow{6}{*}{$\begin{array}{c}\text { Uniform } \\
\text { Distribution }\end{array}$} & \multirow{6}{*}{3,000} & \multirow{6}{*}{1000} & 12 & & 17.55012 & 1.61 & 16.92778 & 20.75 & 7 & 3.55 \\
\hline & & & 14 & & $20.80110 \mid$ & 1.81 & 20.05538 & 12.38 & 6 & 3.59 \\
\hline & & & 16 & & $23.64962 \mid$ & 2.00 & $|22.69465|$ & 9.88 & 8 & 4.04 \\
\hline & & & 18 & 3 & $26.07906 \mid$ & 2.23 & $25.23600 \mid$ & 6.25 & 7 & 3.23 \\
\hline & & & 20 & & $28.59153 \mid$ & 2.45 & $|27.45749|$ & 4.17 & 7 & 3.97 \\
\hline & & & 22 & & 30.51047 & 2.67 & 29.41102 & 1.97 & 6 & 3.60 \\
\hline \multirow{6}{*}{$\begin{array}{c}\text { Normal } \\
\text { Distribution }\end{array}$} & \multirow{6}{*}{3,000} & \multirow{6}{*}{1000} & 12 & & 17.65475 & 1.59 & 16.85623 & 29.66 & 6 & 4.52 \\
\hline & & & 14 & & 20.93491 & 1.81 & 20.30601 & 18.66 & 9 & 3.00 \\
\hline & & & 16 & & 23.82211 & 1.99 & 23.06686 & 6.47 & 7 & 3.17 \\
\hline & & & 18 & 3 & 26.29432 & 2.24 & 25.36438 & 5.03 & 7 & 3.54 \\
\hline & & & 20 & & 28.62054 & 2.42 & $|27.56436|$ & 3.67 & 7 & 3.69 \\
\hline & & & 22 & & $30.57049 \mid$ & 2.64 & 29.41102 & 2.97 & 6 & 3.79 \\
\hline
\end{tabular}

3. The scalability of the $O M M A$ with respect to the number of records per group.

The scalability of the $O M M A$ and the $M D A V$ regarding the group size has 


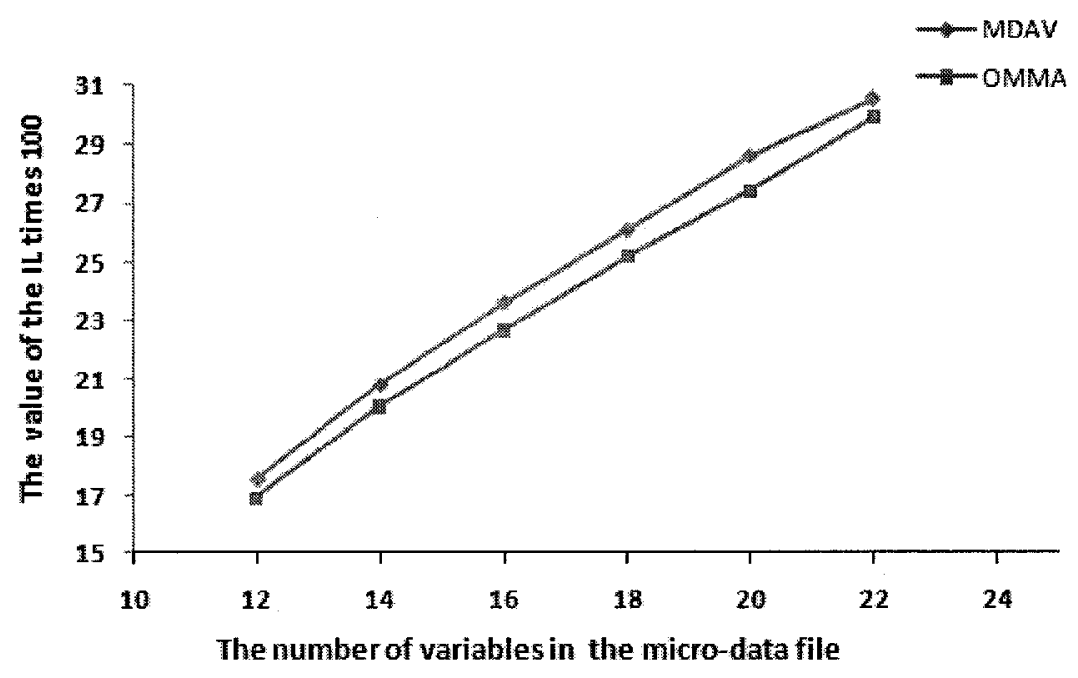

Figure 4.9: The improvement of the $O M M A$ in reducing the percentage value of the $I L$ as a function of the dimension of the data set using uniformly distributed data when $k=3$ and $n=3,000$.

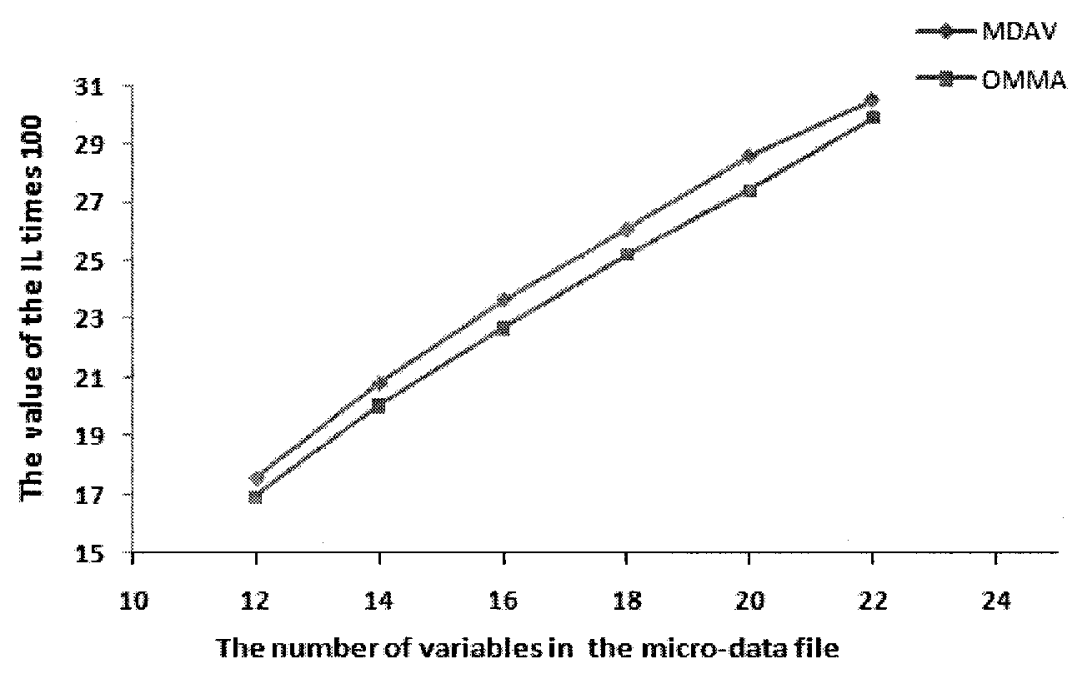

Figure 4.10: The improvement of the $O M M A$ in reducing the percentage value of the $I L$ as a function of the dimension of the data set using normally distributed data when $k=3$ and $n=3,000$. 


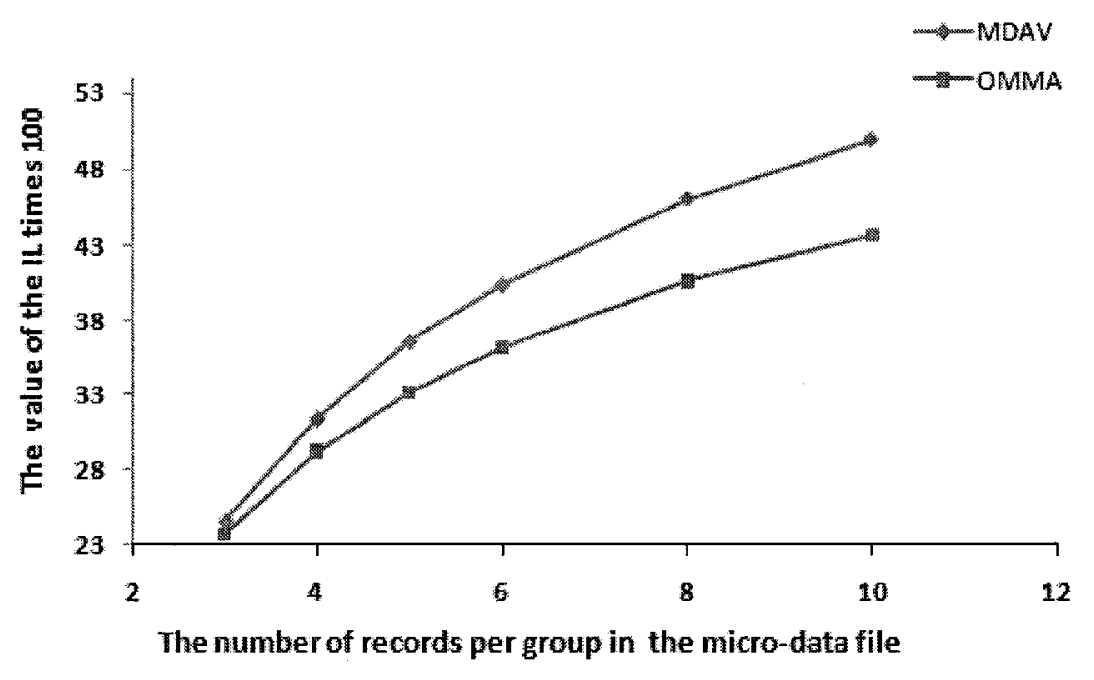

Figure 4.11: The improvement of the $O M M A$ in reducing the percentage value of the $I L$ as a function of the number of records per group using uniformly distributed data when $n=2,400$ and $d=16$.

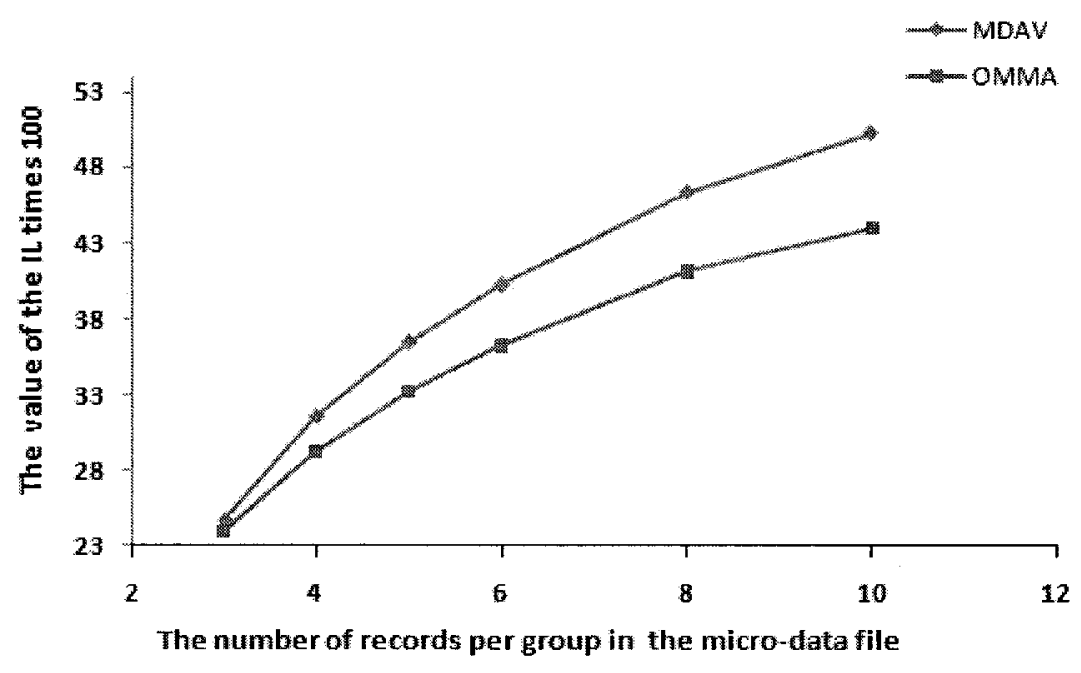

Figure 4.12: The improvement of the $O M M A$ in reducing the percentage value of the $I L$ as a function of the number of records per group using normally distributed data when $n=2,400$ and $d=16$. 
Table 4.7: Comparison of the percentage of the $I L$ and the computation time between the $M D A V$ and the $O M M A$ on simulated uniform and normal data sets for multivariate methods. This table investigates the scalability with respect to the number of records per group.

\begin{tabular}{|c|c|c|c|c|c|c|c|c|c|c|}
\hline \multirow{2}{*}{$\begin{array}{c}\text { Data } \\
\text { Set }\end{array}$} & \multirow{2}{*}{$\begin{array}{l}\text { No. of } \\
\text { Records }\end{array}$} & \multirow{2}{*}{$\begin{array}{l}\text { No. of } \\
\text { Groups }\end{array}$} & \multirow{2}{*}{ Dim. } & \multirow{2}{*}{$\begin{array}{c}k \\
\text { value }\end{array}$} & \multicolumn{2}{|c|}{$M D A V$} & \multicolumn{3}{|c|}{$O M M A$} & \multirow{2}{*}{$\begin{array}{c}\text { Improv. } \\
(\%)\end{array}$} \\
\hline & & & & & IL & Time & $\mathrm{IL}$ & Time & Sonverge & \\
\hline \multirow{7}{*}{$\begin{array}{c}\text { Uniform } \\
\text { Distribution }\end{array}$} & \multirow{7}{*}{2,400} & 800 & \multirow{7}{*}{16} & 3 & 24.64686 & 1.27 & 23.71665 & \begin{tabular}{|l|l|}
5.13 \\
\end{tabular} & 6 & 3.77 \\
\hline & & 600 & & 4 & 31.39147 & 1.28 & 29.17778 & 7.28 & 7 & 7.05 \\
\hline & & 480 & & 5 & 36.55690 & 1.30 & 33.12187 & $7 \mid 10.42$ & 7 & 9.40 \\
\hline & & 400 & & 6 & 40.37307 & 1.30 & 36.19842 & 15.89 & 9 & 10.34 \\
\hline & & 300 & & 8 & 46.01553 & 1.30 & 40.65105 & 19.64 & 7 & 11.66 \\
\hline & & 240 & & 10 & 49.91325 & 1.30 & 43.61896 & 328.50 & 7 & 12.61 \\
\hline & & 200 & & 12 & 53.22947 & 1.30 & 46.16533 & 37.58 & 7 & 13.27 \\
\hline \multirow{7}{*}{$\begin{array}{c}\text { Normal } \\
\text { Distribution }\end{array}$} & \multirow{7}{*}{2,400} & $\overline{80}$ & \multirow{7}{*}{16} & 3 & 24.80187 & 1.28 & 24.00719 & \begin{tabular}{|l|l|}
93.71 \\
\end{tabular} & 6 & 3.20 \\
\hline & & 600 & & 4 & 31.68612 & 1.31 & 29.27261 & 8.19 & 10 & 7.62 \\
\hline & & 480 & & 5 & 36.54596 & 1.33 & 33.24953 & 8.23 & 8 & 9.02 \\
\hline & & 400 & & 6 & 40.23421 & 1.31 & 36.30028 & 13.55 & 8 & 9.78 \\
\hline & & 300 & & 8 & 46.32321 & 1.30 & 41.14944 & 421.86 & 9 & 11.17 \\
\hline & & 240 & & 10 & 50.22509 & 1.33 & 43.99177 & 727.86 & 7 & 12.41 \\
\hline & & 200 & & 12 & 52.78059 & 1.32 & 46.64860 & 37.38 & 7 & 11.62 \\
\hline
\end{tabular}

also been studied for both the uniform and the normal data sets, where the group sizes were $3,4,5,6,8,10$, and 12 , and for the data set cardinality of 2,400 , with the dimensionality of 16 , as shown in Table 4.7. Here, the value of the $I L$ increases with the number of records per group. This is a fair observation, because having many records in the group tends to minimize the within-group similarity, and to simultaneously maximize the similarity between the groups. This also tends to increase the value of the $I L$. In the $O M M A$ the computational time required to micro-aggregate the records increases with the number of records per group. This too can be justified because when the $O M M A$ forces the records to migrate to another group, choosing the record is not an easy job, as it depends on a comprehensive study of the effect of migrating every other possible record- which equals $(k-1)$ possibilities for Case 2 , and $2(k-1)$ possibilities for Case 3. As opposed to this, the computational time is fixed in the $M D A V$ strategies (because it depends only on the size of the data set and the dimensionality). Thus, obviously, increasing the group size 
tends to increase the efficiency of the $O M M A$ by leading to a minimum value of the $I L$, as opposed to the $M D A V$. In the uniform data set the percentage of improvement for the $I L$ is as high as $13.27 \%$, which is obtained when the group size equals 12 . As opposed to this, this improvement reaches $12.41 \%$ for the normal data set, when the group size equals 10. Figures 4.11 and 4.12 show that both schemes possess similar trends for the $I L$ as a function of the group size, and that the value of the $I L$ is proportional to the number of records per group.

\subsection{Conclusions}

In this chapter we have presented, to our knowledge, the first reported $L A$-based solution to the $M A P$, which is known to be $N P$-hard. We have shown that our newly devised scheme, the $O M M A$ can successfully be used to micro-aggregate a multi-variate micro-data file. The $O M M A$ competes in a superior manner to the state-of the art MDAV and MST methods in minimizing the loss in the information for such data. The percentage of improvement reaches up to $13 \%$ when compared to the $M D A V$ scheme on real-life and simulated data sets. The proposed strategy also scales well with respect to the size of the data set, the dimensionality, and the group size. By defining a score index, $S I$, as a composite measure involving the $I L$ and $D R$, we see that proposed strategy also obtains a minimum score value when compared to the $M D A V$ method. This indicates that the $O M M A$ technique is probably the best $M A T$ not only from the $I L$ perspective, but also from the viewpoint of a measure which is as a combination of the $I L$ and $D R$. Therefore, the $O M M A$ can thus be highly recommended for advantageous micro-aggregation. 


\section{Chapter 5}

\section{Using Neural Methods to Solve the MAP}

\subsection{Introduction}

This chapter ${ }^{1}$ presents a possibly pioneering endeavour to tackle the $M A P$ in secure statistical databases, by resorting to the principles of associative Neural Networks $(N N)$. The prior art has improved the available solutions to the $M A T$, by incorporating proximity information, and this is done by recursively reducing the size of the data set by excluding points which are farthest from the centroid, and those which are closest to these farthest points. Thus, although the method is extremely effective, arguably it uses only the proximity information, while ignoring the mutual Interaction between the records. In this chapter, we argue that inter-record relationships can be quantified in terms of two entities, namely their "Association" and "Interaction". This means that the records which are not necessarily close to each other may

\footnotetext{
${ }^{1} \mathrm{~A}$ preliminary version of some of the results from this chapter appeared in the Proceedings of $\mathrm{ICICS}^{\circ} 07$, the 9th International Conference on Information and Communications Security, in Zhengzhou, China, in December 2007 [107]. The journal version of these results is currently under review.
} 
still be "grouped" because their mutual Interaction could be significant. Based on the theoretically sound principles of NNs, we believe that the proximity information can be quantified using the mutual Association, and their mutual Interaction can be quantified by invoking transitive-closure like operations on the latter. By repeatedly invoking the inter-record Associations and Interactions, the records are grouped into sizes of cardinality " $k$ ", where $k$ is the security parameter in the algorithm. Our experimental results, which are done on artificial data and on the benchmark real-life data sets, demonstrate that the newly proposed method is superior to the state-of-the-art not only from the $I L$ perspective, but also when it concerns a criterion involving a combination of the $I L$ and the $D R$.

The structure of this chapter is as follows: We first present a full description of the Associative Cluster Neural Network algorithm, which is used in unsupervised classification, in Section 5.2. In Section 5.3 the Interactive-Associative Micro-Aggregation Technique is presented informally and algorithmically. Then, in Section 5.4, we present the results of experiments we have carried out for synthetic and real data sets. The chapter finishes in Section 5.5 with some conclusions.

\subsubsection{Contribution of the Chapter}

As mentioned in Chapter 2 and 4 , this problem in its multi-variate setting is known to be $N P$-hard [103], and has been tackled using different approaches such as hierarchical clustering [45, 91, 92], genetic algorithms [45, 91, 92, 128], graph theory [74, 84], fuzzy clustering $[57,133]$ and machine learning [64]. As we know from our previous discussions, all the reported heuristic MATs, seek to minimize the value of the $I L$. Although minimizing the loss in the data utility is an important issue, we again argue that maintaining a happy medium between the $I L$ and $D R$ is also crucial $[27,49,60,93]$. In this chapter, we shall argue that a good $M A T$ should be capable of minimizing the $I L$, and yet be able to attain to suitable value for a well-defined composite measure, SI (as mentioned in Chapter 4). Our aim is to find a good strategy to optimize the latter. Observe that the $D R$ in an $M A T$ is solely determined 
by the minimum group size parameter $k$, namely, by ensuring $k$-anonymity, where each data subject is distinguishable from at least $k-1$ other data subjects $[39,60]$.

In general, minimizing the $I L$ directly follows maximizing the similarity between records in each group. The state-of-art MATs depend on utilizing the "Euclidean" distance, which serves as the criterion playing a central role in estimating the similarity between the records. However, this distance function does not completely capture the appropriate notion of similarity for any data set. Our position is that the notion of similarity should be measured by using a metric that also unravels the relationship between the inter-records. We believe that this can be quantified in terms of two quantities, namely the mutual "Association" between the individual records and their mutual "Interaction". We propose to measure these quantities using Association Similarity Rules ${ }^{2}$ (ASRs). In this context, we mention that the concepts of Association and Interaction are derived from the Associative Cluster Neural Network $(A C N N)$, which estimates the similarity between neurons by building a dynamic model evaluated through the interaction between the neurons inside each group, and the interaction among the groups themselves.

The main contribution of this chapter is to integrate the foundational concepts of $A S R s$ with $M A T s$ so as to devise a new strategy for estimating the similarity. This new method demonstrates that the $I L$ can be reduced taking two measurements into consideration. First of all, we consider the mutual Association between the records. Secondly, and more importantly, we also consider the mutual Interaction between the records by using a transitive-closure like operation when $k \geq 3$. This, in turn, is achieved by invoking our newly proposed Interactive-Associative Micro-Aggregation Technique (IAMAT). The effect of these considerations can be seen to minimize the $I L$ by up to $13 \%$ when compared to the state-of-the-art. Apart from this, the IAMAT also yields the best reported values for the above mentioned index, the $S I$. We argue that the applicability of the new strategy in estimating the similarity provides a promising strategy to effectively protect sensitive data in the micro-data

\footnotetext{
${ }^{2}$ Association Similarity Rules are well-known data mining techniques used to discover the relationships between patterns in different application domains $[6,7,9,68,87,116]$.
} 


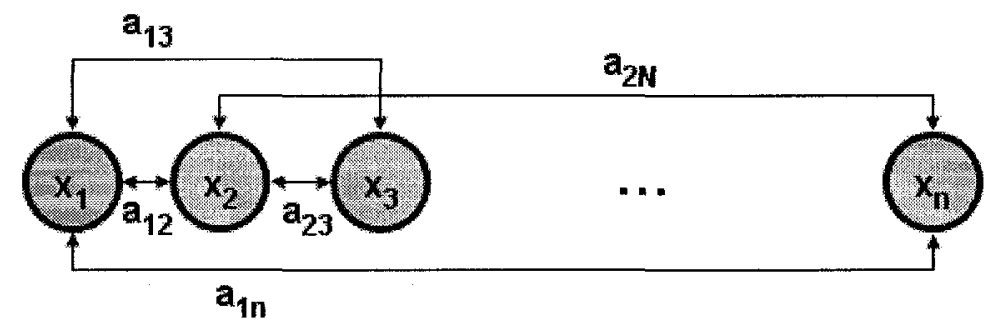

Figure 5.1: The structure of ACNN

file not only based on minimizing the value of the $I L$ but also on offering the best trade-off between the $I L$ and $D R$.

\subsection{Associative Clustering Neural Network $(A C N N)$}

The Associative Cluster Neural Network $(A C N N)$ was proposed [148] as a recurrent $N N$ model that dynamically evaluates the Association of any pair of patterns through the Interaction between them and the group of patterns. The $A C N N$ possesses many attractive features, such as its simple structure, its respective learning mechanism, and its efficient clustering strategy, which uses the Association as a new similarity measure. Its superiority in clustering and analyzing gene expression data has also been demonstrated [149]. The rationale behind this superiority probably lies in the inherent advantages of $A S R s$, that possess the potential to ensure that the similarities between patterns within the same cluster increase, whereas the similarities between different clusters decrease.

The $A C N N$ initializes the Association between any two neurons by evaluating the relationship between them and by setting the learning ratio, $\alpha$, to the most suitable value. The learning ratio should guarantee that the initial association is large when the distance (i.e., proximity in the feature space) between the patterns is small. The $A C N N$ studies the Interaction level of each pair of patterns based on the 
Association made by the other patterns, and defines the similarity threshold which ensures a robust performance. The association value between any two patterns has to be updated based on the result of the Interaction level, and this is, in turn, scaled by using the well-known sigmoid function. This procedure has to be iteratively executed until there is no noticeable change in the successive Associations. Subsequently, the $A C N N$ constructs the cluster characteristic matrix to describe the cluster property at the end of the learning phase, after which it determines the number of clusters and labels the patterns with the index of the cluster that they belong to. We describe the structure and operation of the $A C N N$, more formally, below.

The structure of the $A C N N$ is depicted in Figure 5.1, which clusters the patterns based on evaluating the similarities between them. In the $A C N N$, each pattern is represented by a neuron, while the similarity between them is measured by their mutual Association denoted as $a_{i j}$, and which satisfies the following conditions:

$$
\begin{aligned}
& \text { - } a_{i j}=a_{j i} \text {. } \\
& \text { - }\left\{\begin{array}{l}
a_{i j}>0: X_{i} \text { and } X_{j} \text { are associated } \\
a_{i j} \leq 0: X_{i} \text { and } X_{j} \text { are unrelated. }
\end{array}\right.
\end{aligned}
$$

The $A C N N$ initializes the association between any two neurons, say $X_{i}$ and $X_{j}$, as follows:

$$
a_{i j}(0)=r\left(X_{i}, X_{j}\right)=e^{-\frac{\left\|X_{i}-x_{j}\right\|^{2}}{\alpha}},
$$

where $X_{i}$ and $X_{j}$ are two patterns, $r()$ is a function that evaluates the relationship between any two patterns, and $\alpha$ reflects the decay coefficient that uses the Mean Square Distance in the exponential function, which, in turn, is utilized to determine the initial association $a_{i j}$ between them. The value of $\alpha$ is assigned so as to guarantee that the initial association is large when the distance between $X_{i}$ and $X_{j}$ is small and vice versa. Typically, it is given by:

$$
\alpha=\frac{\sqrt{n}}{\frac{1}{n}\left(\sum_{i=1}^{n}\left\|X_{i}-\frac{1}{n}\left(\sum_{i=1}^{n} X_{i}\right)\right\|^{2}\right)} .
$$


Learning the successive Association between the patterns is done through a number of learning cycles (or epochs). In the cycle with index ' $t$ ', the training of the $N N$ simulates the Interactions of each pair $\left\langle X_{i}, X_{j}\right\rangle$, and this is based on the Associations made by the other patterns on each of them. In other words, for a pattern $X_{p}$, the $A C N N$ attempts to associate $X_{i}$ and $X_{j}$ with values $a_{p i}$ and $a_{p j}$, respectively. If the values of the Association are greater than $0, X_{p}$ has the potential to be in the same cluster containing the pair $\left\langle X_{i}, X_{j}\right\rangle$, where the latter is given as:

$$
y_{i j}(t)= \begin{cases}\sum_{p=1}^{n} a_{i p}(t-1) \otimes a_{p j}(t-1) & p \neq i, j \text { and } i \neq j \\ 0 & i=j\end{cases}
$$

and in which

$$
u \bigotimes v= \begin{cases}u \times v & u>0 \text { and } v>0 \\ 0 & \text { otherwise. }\end{cases}
$$

The association value between any two patterns has to be updated based on the result of the Interaction level, as follows:

$$
a_{i j}(t)= \begin{cases}\varphi\left(y_{i j}(t)\right) & i \neq j \\ 1 & i=j\end{cases}
$$

where $\varphi$ is the sigmoid function given as:

$$
\varphi\left(y_{i j}(t)\right)=\frac{e^{y_{i j}(t)-\theta_{i j}(t)}-1}{e^{y_{i j}(t)-\theta_{i j}(t)}+1}
$$

and where $\theta$ is a similarity threshold that ensures that the characteristics of the unsupervised learning are preserved. The interesting issue here is not one of having a fixed threshold. Rather, each threshold value is derived by making use of the means of the mutual Interactions over all the data points to adjust the sigmoid function, and to thus attain a robust performance. To achieve this, $\theta_{i j}$ is defined as:

$$
\theta_{i j}(t)=\frac{1}{2(n-1)} \sum_{p=1}^{n}\left(y_{p i}(t)+y_{p j}(t)\right)
$$


All the above equations ensure that the similarities within the same cluster increase, whereas the similarities among different clusters decrease as the dynamic system evolves.

The learning process is terminated if the change in the successive Associations is less than a user-defined threshold. In other words, the learning terminates when $E(t) \approx 0$, where,

$$
E(t)=\frac{1}{2} \sum_{i=1}^{n} \sum_{j=1}^{n}\left|a_{i j}(t)-a_{i j}(t-1)\right|
$$

The cluster characteristic matrix, $\mathcal{M}=\left[m_{i j}\right]$, that describes the clustering property at the end of the $A C N N$ learning phase, is defined in terms of its components as:

$$
m_{i j}= \begin{cases}1 & a_{i j}>0 \\ 0 & a_{i j} \leq 0\end{cases}
$$

It can be seen that each pattern $X_{i}$ has its cluster characteristic vector $M_{i}=$ $\left\{m_{i 1}, m_{i 2}, \ldots, m_{i n}\right\}$ that records its cluster property by identifying the associated patterns in the same group. The number of clusters is determined as $C=\operatorname{rank}(\mathcal{M})-|\Psi|$, where $\operatorname{rank}(\mathcal{M})$ is the rank of the matrix $\mathcal{M}$, and $|\Psi|$ is the cardinality of rejected patterns $(\Psi)$, (i.e., those which are isolated from all the other patterns), and is defined ${ }^{3}$ as:

$$
\Psi=\left\{M_{i} \mid \sum_{p=1}^{n} m_{p i}=1 ; i=1, \ldots, n\right\} .
$$

Each pattern will be labeled with the index of the cluster it belongs to. This is done by first excluding the rows of $\mathcal{M}$ which are repetitive, yielding the matrix $\overline{\mathcal{M}}$ of size $C \times n$. The rows of $\overline{\mathcal{M}}$ satisfy:

$$
\overline{\mathcal{M}}_{L}=\left\{M_{i} \mid \sum_{p=1}^{n} m_{p i}>1 ; M_{i} \neq M_{j} \quad i, j=1, \ldots, C ; i \neq j\right\} .
$$

\footnotetext{
${ }^{3}$ This is equivalent to: $\Psi=\left\{M_{i} \mid m_{i i}=1 ; m_{p i}=0 \quad \forall p \neq i\right\}$.
} 
Any pattern $X_{i}$ in the data set will be labeled as

$$
\Theta\left(X_{i}\right) \begin{cases}j & \text { if } \exists M_{j} \in \overline{\mathcal{M}}_{L}, \text { s.t. } m_{j i}=1 \\ 0 & \text { otherwise. }\end{cases}
$$

where $\Theta$ is a labeling function in which 0 represents no label, implying that $X_{i}$ is a rejected pattern without an associated label.

The $A C N N$ learning mechanism is algorithmically described below in Algorithm 11.

\subsection{Interactive-Associative Micro-Aggregation Technique $(I A M A T)$}

The state-of-the-art MATS use a proximity function, and in particular, the Euclidean distance, to measure the similarity between records. To the best of our knowledge, the combination of the Association and the Interaction between individual records has not been taken into consideration while micro-aggregating the data file. We now discuss how these two criteria are applicable to micro-aggregate the data file so as to further minimize the $I L$.

\subsubsection{Inadequacy of Using the $A C N N$ Directly}

Although the basic $A C N N$ dynamically evaluates the Association and the Interaction between the patterns, it is not directly applicable to the $M A P$ in its virgin form. We shall now explain how the principles motivating the $A C N N$ can be extended to solve the MAP. 
Algorithm 11 Associative Cluster Neural Network (ACNN)

Input: A set of patterns, where each pattern represents a neuron.

Output: The set of labeled patterns with the cluster number.

Method:

1: Compute the decay coefficient $\alpha=\frac{\sqrt{n}}{\frac{1}{n}\left(\sum_{i=1}^{n}\left\|X_{i}-\frac{1}{n}\left(\sum_{i=1}^{n} X_{i}\right)\right\|^{2}\right)} . \quad \alpha$ determines the decay in the exponential function in the association function given below.

2: Initialize the association value between every pair of neurons $a_{i j}(0)=e^{-\frac{\left\|x_{i}-x_{j}\right\|^{2}}{\alpha}}$.

3: $t=0$.

4: Repeat

5: $t=t+1$.

6: Stimulate all the interactions on each pair, say $\left\langle X_{i}, X_{j}\right\rangle$, as follows:

$$
\begin{aligned}
& y_{i j}(t)= \begin{cases}\sum_{p=1}^{n} a_{i p}(t-1) \otimes a_{p j}(t-1) & p \neq i, j \text { and } i \neq j \\
0 & i=j\end{cases} \\
& \text { where } u \otimes v= \begin{cases}u \times v & u>0 \text { and } v>0 \\
0 & \text { otherwise. }\end{cases}
\end{aligned}
$$

7: Compute the dynamic similarity threshold between every pair of neurons:

$$
\theta_{i j}(t)=\frac{1}{2(n-1)} \sum_{p=1}^{n}\left(y_{p i}(t)+y_{p j}(t)\right) \text {. }
$$

8: Compute the sigmoid function between every pair of neurons as $\varphi\left(y_{i j}(t)\right)=\frac{e^{y_{i j}(t)-\theta_{i j}(t)}-1}{e^{y_{i j}(t)-\theta_{i j}(t)}+1}$.

9: Update the association value between every pair of neurons based on the Associations made by other neurons linked with them as follow: $a_{i j}(t)=$ $\begin{cases}\varphi\left(y_{i j}(t)\right) & i \neq j \\ 1 & i=j .\end{cases}$

10: Compute $E(t)=\frac{1}{2} \sum_{i=1}^{n} \sum_{j=1}^{n}\left|a_{i j}(t)-a_{i j}(t-1)\right|$ as the change in successive Associations.

11: $\operatorname{Until}(E(t) \approx 0)$

12: Compute the cluster characteristic matrix, which describes the cluster property at the end of the $A C N N$ learning phase. It is built as follows: $m_{i j}=$ $\begin{cases}1 & a_{i j}>0 \\ 0 & a_{i j} \leq 0\end{cases}$

13: Determine the rejected patterns, $\Psi=\left\{M_{i} \mid \sum_{p=1}^{n} m_{p i}=1 \quad i=1, \ldots, n\right\}$. The rejected patterns do not associate with any other pattern.

14: Calculate the number of clusters $C=\operatorname{rank}(\mathcal{M})-|\Psi|$.

15: Select $C$ different cluster characteristic vectors $\overline{\mathcal{M}}_{L}=\left\{M_{i} \mid \sum_{p=1}^{n} m_{p i}>1 ; M_{i} \neq M_{j} \quad i, j=1, \ldots, C ; i \neq j\right\}$.

16: Label each pattern with the indices of the cluster it belongs to as: $\Theta\left(X_{i}\right)\left\{\begin{array}{ll}j & \text { if } \exists M_{j} \in \overline{\mathcal{M}}_{L}, \text { s.t. } m_{j i}=1 \\ 0 & \text { otherwise. }\end{array}\right.$.

17: Return the labeled patterns with the index of the clusters they belong to.

18: End Algorithm Associative Cluster Neural Network ( $A C N N)$ 


\subsubsection{Feature Values}

In a neural setting, the weights of the neurons are updated based on the relative relationship between them. These weights are usually updated by gradient-like considerations, so that a change in the weights leads to a better classifications or performance. Consequently, in all such networks (including the $A C N N$ ) the weights could be both positive or negative, quite simply because increasing the values of certain features may have a negative impact on the optimization problem.

As opposed to this, it is meaningless to have weights that are negative in the MAP. This is because the fundamental reason for tackling the problem is to determine how the records are associated with each other, and clearly, the concept of the records being negatively associated is irrelevant. Thus, if we are to use the principles of the $A C N N$ to solve the $M A P$, it is imperative that the weights are never allowed to become negative. Rather, we would prefer that they stay within the interval $[0,1]$. This is achieved by, first of all, computing the initial Association using the distances, and secondly, by updating the Interactions only as long as they are non-negative. This is achieved by resorting to a single-shot training phase, as will be explained presently.

\subsubsection{Ineffectiveness of Sigmoidal Mappings}

When Minsky suggested the weakness of the Perceptron, he showed that it was incapable of resolving the basic XOR problem. However, the field of $N N s$ received a huge "boost" when it was discovered that if these primitive neural units were cascaded and interconnected, the discriminant could be arbitrarily complex. To effectively model the switching and clipping effects in such complex domains, researchers introduced functions such as the sigmoidal function whose purpose was to transform the input space using highly non-linear mappings.

It is our position that such switching and clipping effects are not pertinent to the 
study of the MAP. The reason for this is quite straightforward: The Associations and the Interactions between the records are, in one sense, related to their relative proximity, and we have no reason to believe that these quantities fall off or change abruptly. Rather, our experience is that these quantities vary smoothly with the relative proximity of the records.

\subsubsection{Transitive-Closure-like Properties}

Obtaining the set of shortest paths on a graph can be achieved by using a transitiveclosure algorithm that traverses all the edges of the graph. In this case, the shortest paths are obtained by using the operation of "Addition" on the weights of the edges along any given path, and invoking the "Minimum" operation over all the possible paths. However, the underlying algorithm has been proven to be much more powerful if it is mapped using the properties of a semi-ring $(S, \oplus, \otimes)$, where (i) $S$ is the set of elements (weights) associated with an edge, (ii) $\bigoplus$ represents an abstract "Addition" operation over the elements of $S$, and (iii) $\otimes$ represents an abstract "Multiplication" operation over the elements of $S$. In particular, if $S$ is the set of reals, and $\bigoplus$ and $\otimes$ represent the arithmetic addition and product operations respectively, the transitive closure algorithm would lead to a matrix multiplication scheme, which is central in determining the multi-step Markov matrix for a Markov chain.

The basic $A C N N$ computes the Interaction between the neurons using the product involving $a_{i p}$ and $a_{p j}$. The total two-step Interaction is thus, effectively, the contribution of the transitive-closure operation of the path from $X_{i}$ to $X_{j}$ via all the possible intermediate nodes, $X_{p}$. In our case, the issue of interest is not the total Interaction between the relevant nodes, but rather the node $X^{*}$ which contributes to the maximal Interaction between $X_{i}$ and $X_{j}$. Thus, unlike the $A C N N$, in our solution, we do not compute the sum of all the Interactions between the nodes. Rather, we report the one which is maximally interacting with the nodes already in the same cluster, say $X_{i}$ and $X_{j}$. This is a fundamental difference between our present scheme and the $A C N N$, because it renders the computations both easier and faster, and is 
yet able to coalesce the nodes based on the inferred interactions.

\subsubsection{One-shot Training}

The final difference between our present scheme and the $A C N N$ is the fact that we have resorted to a one-shot "training" mechanism. This is atypical for most $N N s$. Indeed, most $N N s$ repeatedly run the $N N$ over the data set till their respective weights converge. Some families of $N N s$ (for example, the Adachi's network [1]) have been reported, which actually yield the final weights using a single pass over the data set.

In our case, we argue that repeatedly running the updating algorithm over the data set is superfluous. Rather, by initially computing the Associations, we are able (by the strategy mentioned in the earlier sub-section) to arrive at the best Interactions. The $A C N N$ requires that the set of associations are then re-computed. But, since these associations are computed based on the relative proximities of the records, and since the interactions are computed based on the latter, it is meaningless, in the case of the MAP, to re-compute the associations. Indeed, if we resorted to doing this, it would lead to weights that are negative which again, as argued above, is unacceptable. It would also lead to the "rejection" of many records - which is inappropriate for the $M A P$. Thus, in the IAMAT, the corresponding matrices are computed in a one-shot manner. Subsequent computations are required only after the learned groups of size $k$ are removed in each learning cycle.

Based on the above principles, we now present the design of our newly-proposed scheme, the IAMAT.

\subsubsection{Design of the IAMAT}

We propose the IAMAT to micro-aggregate the records in the data set by using a new methodology to evaluate the similarity between them. This similarity is intuitively 
expressed by their inter-record relationships, and is estimated by measuring the "Association" and "Interaction" as modeled in the $A C N N$. The resulting measurements are similar to the ones that cluster the records based on the distance between them. Consequently, instead of merely assigning relatively "close" records to be in the same group, we choose to "estimate" the Association and the Interaction between them, and if the combination of these indexes is relatively high, we assign them to be in the same group. Otherwise, we determine that they should be in two different groups. We believe that using this pair of measurements will help to achieve a more robust performance than other existing measures, which is a claim that we have verified. From a top level, we can describe it as below.

The IAMAT is a consequence of incorporating the above considerations into the elegant $M D A V$ strategy. Consider the IAMAT for any specific value of $k$. The $I A M A T$ uses the centroid of the data set to relatively determine the least associated record, say $X_{r}$. Subsequently, we achieve a quick search to obtain the record that is most associated to $X_{r}$, say $X_{s}$. After this, we propose to choose $k-2$ records based on the mutual Interaction between each record inside the group and the remaining unassigned records. Consequently, the next step consists of creating a cluster that comprises of the associated pair $\left\langle X_{r}, X_{s}\right\rangle$ and the most interactive $k-2$ records. At the end of this stage, the cluster is micro-aggregated and removed from the original data set. The above steps are iteratively repeated until no more that $k-1$ records remain in the original data set. The IAMAT terminates by assigning the remaining unassigned records to the last group. The scheme is algorithmically described below in Algorithm 12, after which each step is explained in greater detail.

Unlike the $M D A V$, instead of measuring the distance between the records, the $I A M A T$ utilizes the association as per the $A C N N$. The $A C N N$ classifies the records as being associated if the value of the Association index, $a_{i j}$, is positivc. Othcrwise the neurons will be classified as being unrelated, leading to its "rejection". Clearly, rejecting records will not comply with the spirit and goal of the MAP whose aim is to minimize the $I L$. We believe that an Association between any pair of records exists regardless of its value, and this could be very small when it is close to zero, or 
Algorithm 12 Interactive-Associative Micro-Aggregation Technique (IAMAT)

Input: The original micro-data file, $\mathcal{D}$, that contians $n$ unassigned records, and the parameter, $k$.

Output: The micro-aggregated micro-data file, $\mathcal{D}^{\prime}$.

Method:

1: Compute the centroid of $\mathcal{D}$ as $\mu=\frac{1}{n} \sum_{i=1}^{n} X_{i}$.

2: Compute the scaling factor $\alpha$ as $\alpha=\frac{\sqrt{n}}{\frac{1}{n}\left(\sum_{i=1}^{n}\left\|X_{i}-\mu\right\|^{2}\right)}$.

3: Compute the association values between $\mu$ and each record, $X_{i}$, as $a_{\mu i}=e^{-\frac{\left\|x_{i}-\mu\right\|^{2}}{\alpha}}$.

4: Initialize the number of groups to zero.

5: while there are more than $(k-1)$ Unassigned records in $\mathcal{D}$ do

6: Increment the number of groups by unity.

7: Initialize the number of records inside the group to zero.

8: Select the least associated Unassigned record, $X_{r}$, to the centroid $\mu$ as follows $X_{r}=$ Min $a_{\mu i}$ and mark it as Assigned record.

9: Compute the association values between $X_{r}$ and each Unassigned record, $X_{i}$, in $\mathcal{D}$.

10: Select the most associated Unassigned record, $X_{s}$, to $X_{r}$ as follows $X_{s}=$ Max $a_{r i}$ and mark it as an Assigned record.

11: Compute the association values between $X_{s}$ and each Unassigned record, $X_{i}$, in $\mathcal{D}$.

12: Add $X_{r}$ and $X_{s}$ to the group and increment the number of records inside the group by two units.

13: $\quad$ while the number of records inside the group is less than $k$ do

14: $\quad$ for all Unassigned records, $X_{p}$, in $\mathcal{D}$ do

15: Initialize the Interaction of $X_{p}, \eta_{p}$, to 1 .

16: for all Assigned records inside the group, $X_{i}$ do

17: $\quad$ Update the value of Interaction as follows $\eta_{p}=\eta_{p} * a_{i p}$.

18: $\quad$ end for

19: $\quad$ end for

20: $\quad$ Let $X^{*}$ be the record which has the highest value for $\eta_{p}$ and mark it as an Assigned record.

21: $\quad$ Add $X^{*}$ into this group and increment the number of records inside the group by unity.

22: $\quad$ Compute the association values between the most interactive record, $X^{*}$ and each Unassigned record, $X_{i}$, in $\mathcal{D}$.

23: end while

24: Remove the present cluster from the set $\mathcal{D}$.

25: end while

26: Assign the remaining Unassigned records to the last group.

27: return the micro-aggregated data file, $\mathcal{D}^{\prime}$.

28: End Algorithm (IAMAT) 
very large when is close to unity. Therefore, the IAMAT quantifies the value of the Association between two records, say $X_{i}$ and $X_{j}$, to belong to the interval $[0,1]$, and this is computed as follows:

$$
a_{i j}=a_{j i}=r\left(X_{i}, X_{j}\right)=e^{-\frac{\left\|X_{i}-X_{j}\right\|^{2}}{\alpha}} .
$$

where $r()$ is the identical function used in the definition of the $A C N N$, which evaluates the relationship between any two records, and which also involves $\alpha$. As mentioned, the value of $\alpha$ is assigned so as to guarantee that the initial Association is large when the distance between $X_{i}$ and $X_{j}$ is small and vice versa. Typically, it is given by:

$$
\alpha=\frac{\sqrt{n}}{\frac{1}{n}\left(\sum_{i=1}^{n}\left\|X_{i}-\frac{1}{n}\left(\sum_{i=1}^{n} X_{i}\right)\right\|^{2}\right)} .
$$

The rationale for incorporating the Association with the Interaction between the records inside a group, is that it leads to more homogeneous groups. The concept of the Interaction turns out to be crucial in forming the cluster, because we believe that merely being close to the farthest records is not a reason that is sufficiently important for any record to be grouped with the most distant one. Rather, we propose that the Interaction with respect to all the records inside the group has to be taken into consideration while clustering the records. As mentioned earlier, the latter is computed by invoking transitive-closure like operations. Finding the most interactive record with the associated pair is achieved by searching for the maximum product of the Association between the unassigned records, say $X_{p}$, and each record in the associated pair, $\left\langle X_{i}, X_{j}\right\rangle$, as follows:

$$
\eta_{i j}= \begin{cases}a_{i p}(t-1) \times a_{p j}(t-1) & p \neq i, j \text { and } i \neq j \\ 0 & i=j\end{cases}
$$

The above equation is valid when $k=3$. By increasing the value of $k$, the transitive-closure is applied by adding one unassigned record at a time. The decision of grouping the unassigned record with other records in the group depends on the 
Interaction of that record with respect to other records inside the group. Logically, the most interactive unassigned record has been chosen as follows:

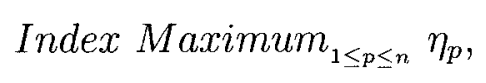

where

$$
\eta_{p}=\prod_{i=1}^{n_{j}} a_{i p}
$$

where $X_{i}$ represents the record inside the group, $G_{j}$, of size $n_{j}$ and $X_{p}$ represents the unassigned record.

\subsection{Experimental Results}

\subsubsection{Data Sets}

The IAMAT has been extensively tested ${ }^{4}$ and the results obtained seem to be very good, where the "goodness" of a scheme refers to the combination of its being efficiently computed, and its offering a good trade-off between the $I L$ and $D R$. We have tested it using the two real-life benchmark reference data sets used in previous studies, and in various simulated data sets obtained using the Matlab's build-in functions. These sets are:

1. The Tarragona data set which contains 834 records with 13 variables [45].

2. The Census data set which contains 1080 records with 13 variables [53].

3. A uniform distribution $(\min =0 ; \max =1000)$.

\footnotetext{
${ }^{4}$ The reader is encouraged to re-visit Section 4.4 of Chapter 4 to see how the $M D A V$ and the new method are compared using the composite Scoring Index, namely $S I$.
} 
4. A normal distribution $(\mu=0 ; \sigma=0.05)$.

5. A uniform distribution ( $\min =0 ; \max =40,000)$ which contains 10,000 records and 16 dimensions.

6. A normal distribution $(\mu=500 ; \sigma=150)$ which contains 6,000 records and 16 dimensions.

While that data sets in items $1,2,5$, and 6 are identical to those in Chapter 4 , the data sets in items 3 and 4 involve vectors with dimensions ranging from 10 up to 80 , and sets of cardinality from 10,000 up to 100,000. The latter data sets were used to investigate the scalability of the IAMAT with respect to the size of the data, its dimensionality, and the number of records per group.

\subsubsection{Results}

For a given value of the security parameter $k$, which represents the minimum number of records per group, we compared the percentage value of the $I L=(S S E / S S T)$ (as defined in Chapter 2) resulting from the IAMAT and the MDAV strategies. It is important to mention that the $M D A V$ was implemented based on the centroid concept and not a diameter concept ${ }^{5}$. All the programs were written in the $C^{++}$ language, and the tests were performed on an Intel(R) Pentium (R)M Processor with the clock speed of $1.73 \mathrm{GHz}$, and with $512 \mathrm{MB}$ of $\mathrm{RAM}$.

Table 5.1 shows the improvement of the solution obtained by using the IAMAT as opposed to the $M D A V$ on the multi-variate real data sets, where all the 13 variables were used simultaneously during the micro-aggregation process. The reduction in the value of the $I L$ attained up to $8 \%$ on the Tarragona data set, and $5.12 \%$ on the Census data set when the group size was equal to 3 . But in the case of the simulated data sets, the improvement in $I L$ reached up to $14.47 \%$ when $k=5$ in the uniform data set,

\footnotetext{
${ }^{5}$ We did not program the $M D A V$ scheme. We are extremely thankful to Dr.Francesc Sebé for giving us his source code.
} 
Table 5.1: Comparison of the percentage of the $I L$ and the computational time between the $M D A V$ and the IAMAT on the real-life data sets (Tarragona and Census), and simulated data sets (Uniform and Normal distributions) for multi-variate methods.

\begin{tabular}{|c|c|c|c|c|c|c|}
\hline \multirow{2}{*}{$\begin{array}{c}\text { Data } \\
\text { Set }\end{array}$} & $\mathrm{k}$ & \multicolumn{2}{|c|}{$M D A V$} & \multicolumn{2}{c|}{ IAMAT } & Improv. \\
\cline { 3 - 6 } & value & $\mathrm{IL}$ & Time & IL & Time & $(\%)$ \\
\hline \hline & 3 & 16.9593 & 0.17 & 15.6023 & 0.31 & 8.00 \\
\hline Tarragona & 4 & 19.7482 & 0.12 & 19.2872 & 0.22 & 2.33 \\
\hline & 5 & 22.8850 & 0.12 & 22.7164 & 0.23 & 0.74 \\
\hline \hline & 3 & 5.6535 & 0.22 & 5.3639 & 0.41 & 5.12 \\
\hline & 4 & 7.4414 & 0.19 & 7.2170 & 0.44 & 3.02 \\
\hline Census & 5 & 8.8840 & 0.17 & 8.8428 & 0.42 & 0.46 \\
\hline & 6 & 10.1941 & 0.17 & 9.9871 & 0.42 & 2.03 \\
\hline \hline & 3 & 22.4608 & 4.18 & 19.9730 & 8.52 & 11.08 \\
\hline Uniform & 4 & 29.1714 & 4.45 & 25.2008 & 7.85 & 13.61 \\
\hline Distribution & 5 & 33.9636 & 4.62 & 29.0478 & 7.48 & 14.47 \\
\hline & 6 & 37.1577 & 4.75 & 32.1441 & 7.18 & 13.49 \\
\hline \hline & 3 & 26.9348 & 0.45 & 24.1245 & 0.92 & 10.43 \\
\hline Normal & 4 & 33.9256 & 0.78 & 30.0154 & 0.81 & 11.53 \\
\hline Distribution & 5 & 39.564 & 0.59 & 34.5886 & 0.87 & 12.58 \\
\hline & 6 & 43.4592 & 0.60 & 38.0086 & 0.75 & 12.54 \\
\hline \hline
\end{tabular}


and it was as high as $12.58 \%$ when $k=5$ in the normal data set. It is, thus, evident that the impact of the group size on the solution is minimized by increasing the number of records per group in the real data stes. To be fair, the computational time required to execute the $I A M A T$ is almost double the computational time required for the $M D A V$, although, in every case, the time was marginal - less than 0.5 second. In term of comparison, we believe that minimizing the loss in the data utility is more important than minimizing the extremely small computational time, especially because the micro-aggregation is usually performed off-line where the additional time requirement is less crucial. However, the question of how the decrcase of $I L$ is related to the increase in the computational time is still open.

Table 5.2: Scoring the MDAV and IAMAT with respect to the $G_{I L}$ and $G_{D R}$ by computing the index $S I$, for $k=3,4$, and 5 by using the Census data set and the simulated Uniform and Normal distributed data sets.

\begin{tabular}{|c|c|c|c|c|c|c|c|}
\hline Data & Creterion & \multicolumn{2}{|c|}{$k=3$} & \multicolumn{2}{c|}{$k=4$} & \multicolumn{2}{c|}{$k=5$} \\
\cline { 3 - 8 } & & $M D A V$ & $I A M A T$ & $M D A V$ & $I A M A T$ & $M D A V$ & $I A M A T$ \\
\hline \hline \multirow{4}{*}{ Census } & $G_{I L}$ & 28.6100 & 22.1500 & 33.2540 & 30.3120 & 38.6720 & 34.9580 \\
\cline { 2 - 8 } & $R L D$ & 60.7133 & 61.8889 & 49.7057 & 51.9070 & 42.0981 & 43.1771 \\
\cline { 2 - 8 } & $I D$ & 1.9815 & 1.8704 & 0.7315 & 0.8611 & 0.2685 & 0.5556 \\
\cline { 2 - 8 } & $G_{D R}$ & 31.3474 & 31.8796 & 25.2186 & 26.3840 & 21.1833 & 21.8663 \\
\cline { 2 - 8 } & $S I$ & 33.8050 & 30.5750 & 36.1270 & 34.6560 & 38.8360 & 36.9790 \\
\hline \hline \multirow{5}{*}{ Uniform } & $G_{I L}$ & 115.4319 & 37.8225 & 116.1198 & 45.1763 & 120.9199 & 53.3052 \\
\cline { 2 - 8 } & $R L D$ & 0.1088 & 1.1875 & 0.0694 & 0.8449 & 0.0602 & 0.5556 \\
\cline { 2 - 8 } & $I D$ & 0.0042 & 4.7194 & 0.0000 & 1.2437 & 0.0000 & 0.4924 \\
\cline { 2 - 8 } & $G_{D R}$ & 0.0565 & 2.9535 & 0.0347 & 1.0443 & 0.0301 & 0.5240 \\
\cline { 2 - 8 } & $S I$ & 57.7442 & 20.3880 & 58.0773 & 23.1103 & 60.4750 & 26.9146 \\
\hline \hline \multirow{5}{*}{ Normal } & $G_{I L}$ & 108.9812 & 61.8348 & 89.0398 & 80.1520 & 110.8694 & 105.2394 \\
\cline { 2 - 8 } & $R L D$ & 0.6494 & 11.8173 & 0.6571 & 6.7410 & 0.2814 & 5.3885 \\
\cline { 2 - 8 } & $I D$ & 0.0085 & 0.8248 & 0.0000 & 0.1987 & 0.0000 & 0.0406 \\
\cline { 2 - 8 } & $G_{D R}$ & 0.3290 & 6.3210 & 0.3285 & 3.4699 & 0.1407 & 2.7145 \\
\hline \hline
\end{tabular}

The other experiments were carried to test the $S I$ of the $M D A V$ and $I A M A T$. Therefore, they have been scored as per the $S I$ index on the simulated data and the Census ${ }^{6}$ data sets, which contains seven key variables: $\operatorname{Var} 1, \operatorname{Var} 2, \operatorname{Var} 3, \operatorname{Var} 5$,

\footnotetext{
${ }^{6}$ Scoring them against the Tarragona data set is meaningless because the latter contains no socalled key variables.
} 
$\operatorname{Var} 10, \operatorname{Var} 11$, and $\operatorname{Var} 12$.

Table 5.2 displays the $S I$ for the $M D A V$ and $I A M A T$ methods for various values of $k$, which was set to be either 3,4 or 5 as per the accepted requirements stated in $[46,115,140]$. As per Eq. (4.2), the $G_{I L}$ was computed by averaging the values of $M_{1}, M_{2}, M_{3}, M_{4}$, and $M_{5}$. In general, the value of the $G_{I L}$ is directly proportional to the number of records per group represented by the value, $k$. Therefore, in the Census data set, the best value of $G_{I L}$ for the IAMAT was obtained when $k=3$, and was equal to $22.15 \%$, while the best value of $G_{I L}$ for the $M D A V$ was $28.61 \%$ when $k=3$. In the context of the simulated data sets, and in general, the value of $G_{I L}$ for the $I A M A T$ is less than half the value that was obtained by using the $M D A V$ (i.e., the value of $G_{I L}$ for the $I A M A T$ in the uniform data set and when $k=3$ was $37.82 \%$, while it was equal to $115.43 \%$ for the $M D A V$ ). Clearly, the IAMAT method preserves the data utility more efficiently than the state-of-the-art, $M D A V$.

We also compared the $G_{D R}$ (which estimates the risk of the data being disclosed) using the $R L D$ and $I D$ techniques. The $R L D$ was computed using distance-based metric computations, where the average value was computed over several scenarios (as mentioned in Chapter 4). Each scenario computed the average of the $R L D$ over all possible $\left(\begin{array}{l}S \\ C\end{array}\right)=\frac{S !}{C !(S-C) !}$ combinations, where $S$ is the number of key variables in the micro-data file, and $C$ is the number of chosen variables that are known to the Intruder. The results (Table 5.2) generally shows that estimating the risk of disclosing the secure information using the $R L D$ method falls inversely "proportional" with the number of records per group represented by $k$. Thus, in the Census data set, the percentage value of the $R L D$ for the $M D A V$ was as low as $60.71 \%$ when $k=3$, $49.70 \%$ when $k=4$, and $42.10 \%$ when $k=5$, while it was $61.89 \%$ when $k=3$, $51.90 \%$ when $k=4$, and $43.18 \%$ when $k=5$ for the IAMAT. Similarly, for the simulated data sets, we report the same fact which is that the $M D A V$ method is more secure than the IAMAT method in estimating the $R L D$. For example, the percentage value of the $R L D$ by using the $M D A V$ on the uniform data set was around $0.1 \%$, while it was around $1.0 \%$ by using the IAMAT. On the other hand, the percentage value of the $I D$ was calculated as the average value for various settings 


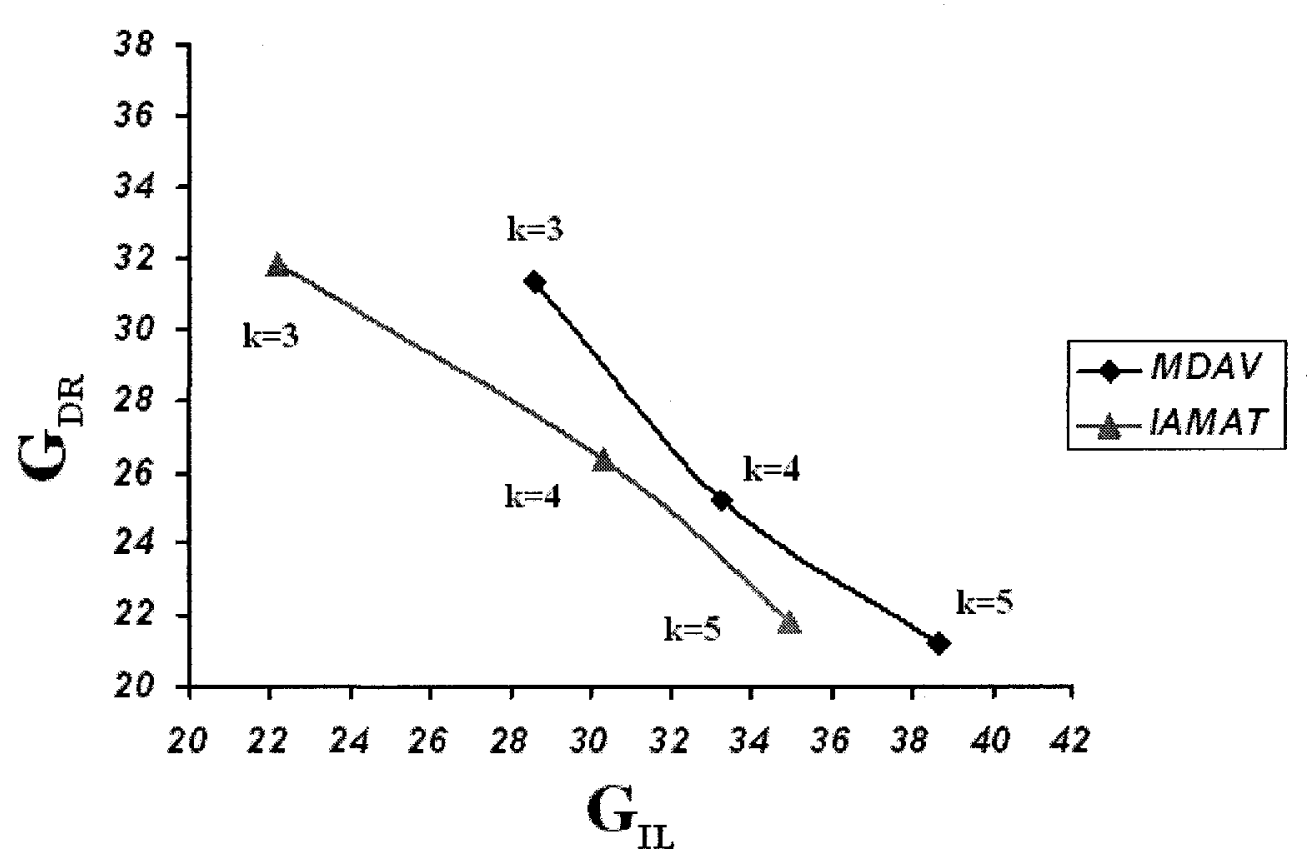

Figure 5.2: The effect of invoking the $M D A V$ and $I A M A T$ on the $G_{I L}$ and $G_{D R}$ indices when $k=3,4$ and 5 for Census data set.

of $P,(1,2, \ldots, 10 \%)$ on the Census data set, when the value of $k$ was assigned to be 3,4 , or 5 . We observe that the minimum percentage value of the $I D$ was equal to $0.27 \%$, which was achieved by invoking the $M D A V$ when $k=5$. As opposed to this, the minimum percentage value of the $I D$ for the IAMAT was almost comparable to $0.56 \%$ when $k=5$. In the simulated data sets, the percentage value of the $I D$ was equal to zero for the IAMAT and $M D A V$. Therefore, the range of values for $P$ was extended to reach up to $30 \%$ of the original size of the data sets. The percentage value of the $M D A V$ remained zero, while the minimum percentage value for the IAMAT reached up to $0.49 \%$ and $0.04 \%$ when $k=5$ in the uniform and normal data sets, respectively.

Finally, the $S I$ value was computed for each $M A T$ based on Eq. (4.4). Observe that a lower score value implies a superior performance. From the table we see that the IAMAT technique has, almost consistently, a better performance index than the 
$M D A V$ not only from the $G_{I L}$ perspective, but also as from the perspective of a combination of the $G_{I L}$ and $G_{D R}$ for different values of $k$. Thus, for example, in Census data set the IAMAT method scores the minimum value that was equal to 30.58 when $k=3$, to 34.66 when $k=4$, and $36.98 \%$ when $k=5$. Besides the I AM AT scores almost half the $S I$ value which was obtained by the $M D A V$ by using the simulated uniform and normal data sets.

An interesting exercise involves studying how the IAMAT and the MDAV compared when "all" the factors coming to play. In other words, it would be good if we could understand the conditions when one algorithm is superior to the other and vice versa. While this can be done in many ways, we have plotted the $G_{I L}$ versus the $G_{D R}$ for the Census data set, in Figure 5.2, for both schemes. This figure presents sets of paired values of $G_{I L}$ and $G_{D R}$ for the respective algorithm for different values of $k$ ranging from 3 to 5 . The user should observe the influence of a masked method as the value of $k$ changes. From the curve we see that the IAMAT optimizes these conflicting criteria in a superior way than the stat-of-the-art $M A D V$ method, because as the value of $k$ increases, the increase of $G_{I L}$ does not effect the value of $G_{D R}$ in the IAMAT method as it does in the case of the MDAV.

We also undertook a comprehensive evaluation of the performance of the IAMAT scheme so as to investigate the scalability of the technique with respect to the cardinality of the data set, its dimensionality and the number of records per group.

\section{- The scalability of the $I A M A T$ with respect to cardinality.}

We tested both the IAMAT and the MDAV schemes using data based on the uniform and the normal distributions with cardinalities ranging from 10,000 records up to 100,000 with 10 variables as shown in Tables 5.3. The percentage of the improvement achieved by invoking the IAMAT in the $I L$, (when the value of $k$ was set to 3 ) ranges from $10.02 \%$ to $11.25 \%$ for the normal distribution, and ranges from $10.47 \%$ to $11.28 \%$ for the uniform distribution. The same experiments were repeated for the value $k=4$. The improvement increased and reached as high as $13.73 \%$ for the normal distribution and $13.67 \%$ for the 
uniform distribution. It is fair to state that the $I A M A T$ requires almost triple the computational time needed to execute the $M D A V$ scheme. Figures 5.3 and 5.4 display similar trends for minimizing the value of the $I L$ as a function of the data set size for uniform and normal distributions, respectively. In general, increasing the size of the data set tends to minimize the $I L$ value. It should be mentioned that the IAMAT was superior to the $M D A V$ in every single case.

Table 5.3: Comparison of the percentage of the $I L$ and the computation time between the $M D A V$ and the IAMAT on simulated data involving the uniform and normal distributions. The results demonstrate the scalability of the IAMAT with respect to the size of the data set (when $k=3$ and $k=4$ ).

\begin{tabular}{|c|c|c|c|c|c|c|c|c|c|c|c|}
\hline \multirow{3}{*}{$\begin{array}{l}\text { Data } \\
\text { Size }\end{array}$} & \multirow{3}{*}{$\begin{array}{c}\mathrm{k} \\
\text { value }\end{array}$} & \multicolumn{5}{|c|}{ Normal Distribution } & \multicolumn{5}{|c|}{ Uniform Distribution } \\
\hline & & \multicolumn{2}{|c|}{$M D A V$} & \multicolumn{2}{|c|}{$I A M A T$} & \multirow{2}{*}{$\begin{array}{c}\text { improv. } \\
(\%)\end{array}$} & \multicolumn{2}{|c|}{$\overline{M D A V}$} & \multicolumn{2}{|c|}{$I A M A T$} & \multirow{2}{*}{ improv. } \\
\hline & & IL & Time & IL & Time & & IL & Time & IL & Time & \\
\hline \multirow[t]{2}{*}{$10 \mathrm{k}$} & $\overline{3}$ & 10.5383 & $\overline{13.53}$ & 9.4821 & 32.96 & 10.02 & 10.5437 & 13.75 & 9.4400 & 33.87 & 10.47 \\
\hline & 4 & \begin{tabular}{|l|}
13.9376 \\
\end{tabular} & 12.05 & 12.2106 & 29.37 & 12.39 & 13.9202 & 12.24 & 12.2069 & 29.96 & 12.31 \\
\hline \multirow[t]{2}{*}{$20 \mathrm{k}$} & 3 & 65 & 54.33 & .0597 & $\overline{135.14}$ & 10.51 & 9.0458 & 58.57 & 8.0903 & 139.59 & 10.56 \\
\hline & 4 & 11.9354 & 48.90 & 10.3653 & 120.37 & 13.15 & \begin{tabular}{|c|}
11.9617 \\
\end{tabular} & 63.15 & 10.4275 & 145.09 & 12.83 \\
\hline \multirow[t]{2}{*}{$30 \mathrm{k}$} & 3 & 8.2177 & 125.75 & $\mid 7.3392$ & $\overline{304.38}$ & 10.69 & 8.2435 & 127.04 & 7.3442 & 311.29 & $\overline{10.91}$ \\
\hline & 4 & 10.8849 & 111.84 & \begin{tabular}{|l|}
9.4488 \\
\end{tabular} & 277.20 & 13.19 & 10.9228 & 143.17 & 9.4976 & 328.02 & 13.05 \\
\hline \multirow[t]{2}{*}{$40 \mathrm{k}$} & 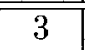 & 7.7352 & 222.23 & 6.8839 & $\overline{547.57}$ & 11.01 & 7.7576 & 226.43 & 6.9063 & 554.01 & 10.97 \\
\hline & 4 & 10.2238 & \begin{tabular}{|l|}
200.42 \\
\end{tabular} & 8.8732 & 494.94 & 13.21 & 10.2733 & 222.74 & 8.9063 & 526.37 & 13.31 \\
\hline \multirow[t]{2}{*}{$50 \mathrm{k}$} & 3 & 7.3644 & $\overline{349.66}$ & 6.5548 & 855.77 & $\overline{10.99}$ & $\mid 7.3724$ & 335.32 & 6.5611 & 867.68 & 11.00 \\
\hline & 4 & \begin{tabular}{|l|}
9.7234 \\
\end{tabular} & 315.04 & 8.4382 & 773.92 & 13.22 & \begin{tabular}{|l|}
9.7694 \\
\end{tabular} & 389.45 & 8.4574 & 903.39 & 13.43 \\
\hline \multirow[t]{2}{*}{$60 \mathrm{k}$} & $\overline{3}$ & 7.0538 & 505.63 & 6.2888 & $\mid 1,247.30$ & 10.85 & $7.0896 \mid$ & 516.40 & 6.2956 & 1275.53 & 11.20 \\
\hline & 4 & 9.3513 & 457.50 & 8.1119 &, 115.69 & 13.25 & 9.3707 & 644.32 & 8.1338 & 1386.01 & 13.20 \\
\hline \multirow[t]{2}{*}{$70 \mathrm{k}$} & 3 & 6.8195 & 693.42 & 6.0700 & ,683.27 & 10.99 & |6.8504 & 723.90 & 6.0822 & 1790.66 & 11.21 \\
\hline & 4 & 9.0358 & 623.89 & 7.8243 &, 520.94 & 13.41 & 9.0776 & 629.73 & 7.8523 & 1500.12 & 13.50 \\
\hline \multirow[t]{2}{*}{$80 \mathrm{k}$} & 3 & 6.6273 & 2909.36 & 5.8972 & $2,194.42$ & 11.02 & 6.6537 & 924.03 & 5.9029 & 2277.30 & 11.28 \\
\hline & 4 & 8.7898 & 809.397 & 7.6063 & $1,981.06$ & 13.46 & 8.8183 & 1002.77 & 7.6247 & 1959.25 & 13.54 \\
\hline \multirow[t]{2}{*}{$90 \mathrm{k}$} & 3 & 6.4592 & $1,152.77$ & 5.7387 & $2,776.69$ & 11.15 & 6.4797 & 1181.84 & 5.7529 & 2855.63 & 11.22 \\
\hline & 4 & 8.5584 & 044.77 & 7.4010 & $2,505.86$ & 13.52 & 8.5885 & 1252.61 & 7.4140 & 2485.42 & 13.68 \\
\hline \multirow[t]{2}{*}{$100 \mathrm{k}$} & 3 & 25 & & 021 & 86 & 11.25 & & & $\overline{6218}$ & 9.00 & 11.14 \\
\hline & 4 & 8.3799 & 12.00 & 1.4290 & 413.98 & 13.73 & 8.393 & & .2400 & 40.12 & 13.67 \\
\hline
\end{tabular}

- The scalability of the $I A M A T$ with respect to dimensionality.

We also tested the IAMAT and the $M D A V$ on the uniform and the normal distributions for various dimensions of the variables ranging from 10 to 80 , 


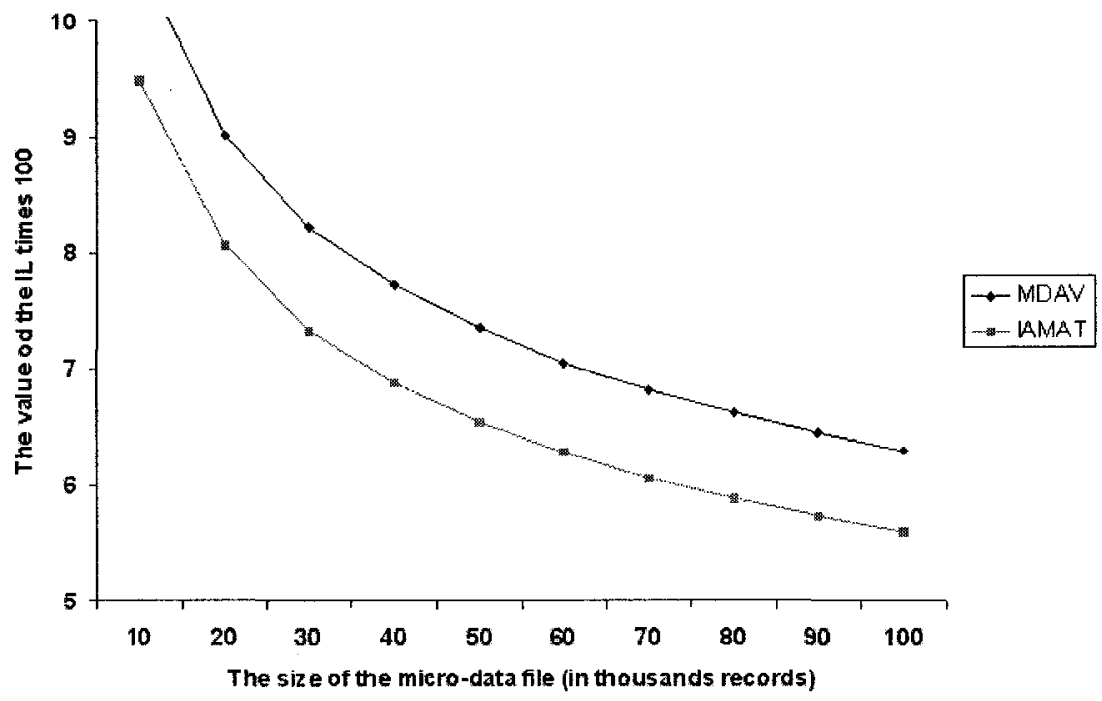

Figure 5.3: The improvement of the IAMAT in reducing the percentage value of the $I L$ as a function of the cardinality of the data set using normally distributed data when $k=3$.

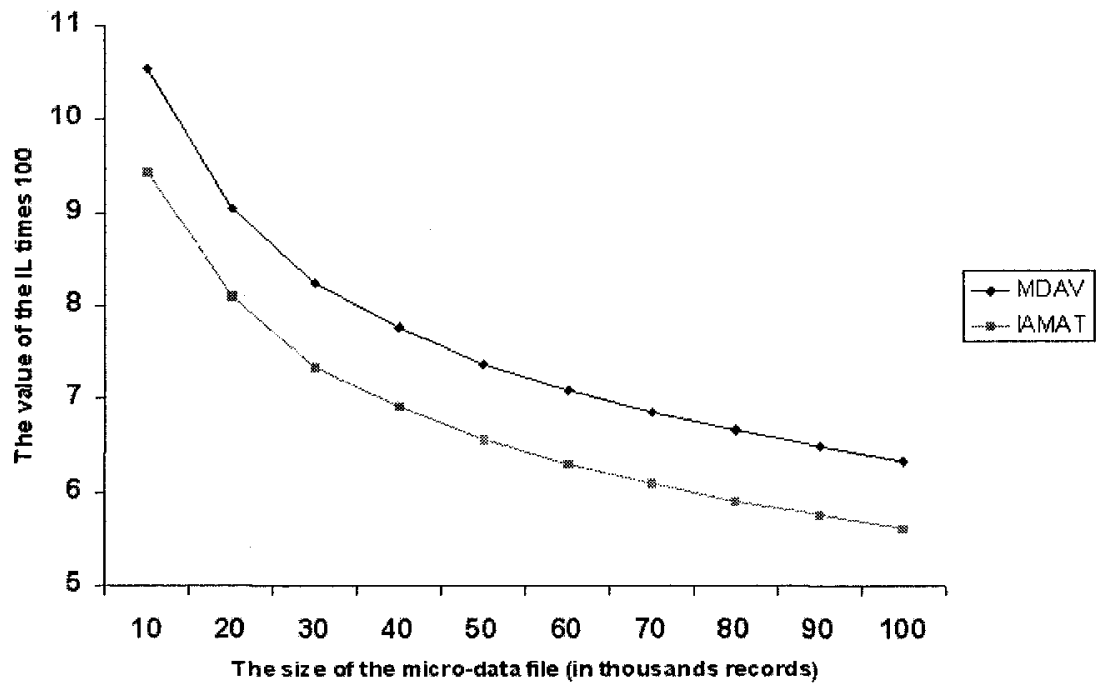

Figure 5.4: The improvement of the $I A M A T$ in reducing the percentage value of the $I L$ as a function of the cardinality of the data set using uniformly distributed data when $k=3$. 
when the data size was set to 10,000 records, and the value of $k$ was set to 3. Table 5.4 shows that the highest percentage of the improvement in the $I L$ was about $10 \%$ for both the uniform and normal distributions. The computational time required to micro-aggregate all the individual records was almost directly proportional to the dimensionality in both schemes, and the required computational time in the IAMAT was almost double the required time for the $M D A V$. Figures 5.5 and 5.6 show that both the IAMAT and MDAV schemes have similar trends for the $I L$ i.e., they are almost proportional to the dimension of the variables used in the micro-aggregation process for both distributions. As expected, increasing the dimensionality implies increasing the loss in the data utility. This is intuitively appealing because, informally speaking, increasing the dimensionality tends to minimize the similarity between the individual records and, at the same time, reduce the Association and the Interaction between the different multi-variate records.

Table 5.4: Comparison of the percentage of the $I L$ and the computation time between the $M D A V$ and the IAMAT on simulated data involving uniform and normal distributions. The results demonstrate the scalability of the $I A M A T$ with respect to the dimensionality of the micro-data file.

\begin{tabular}{|c|c|c|c|c|c|c|c|c|c|c|}
\hline \multirow{3}{*}{$\begin{array}{l}\text { Number } \\
\text { of } \\
\text { variables }\end{array}$} & \multicolumn{5}{|c|}{ Normal Distribution } & \multicolumn{5}{|c|}{ Uniform Distribution } \\
\hline & \multicolumn{2}{|c|}{$M D A V$} & \multicolumn{2}{|c|}{ IAMAT } & \multirow{2}{*}{$\underset{(\%)}{i m p r o v .}$} & \multicolumn{2}{|c|}{$\overline{M D A V}$} & \multicolumn{2}{|c|}{$I A M A T$} & \multirow{2}{*}{$\begin{array}{c}\text { improv. } \\
(\%)\end{array}$} \\
\hline & IL & Time & $\overline{\mathrm{IL}}$ & Time & & $\overline{\mathrm{IL}}$ & Time & IL & Time & \\
\hline$\overline{10}$ & 10.5383 & 13.53 & 9.4821 & $\overline{30.14}$ & 10.02 & 10.5437 & 13.75 & 9.4400 & 33.87 & 10.47 \\
\hline 20 & 24.3766 & 24.30 & 22.3577 & 41.26 & 8.28 & 24.4692 & 25.0 & 22.2454 & 46.40 & 9.09 \\
\hline 30 & 32.6044 & 35.50 & 29.9938 & 69.79 & 8.01 & 32.5113 & 36.67 & 29.9711 & 60.00 & 7.81 \\
\hline 40 & 37.5679 & 47.00 & 34.8732 & 64.98 & 7.17 & 37.4627 & 47.18 & 34.8052 & 72.68 & 7.09 \\
\hline 50 & 40.8705 & 40.87 & 38.2173 & 67.87 & 6.49 & 40.8427 & 58.78 & 38.2470 & 85.98 & 6.36 \\
\hline 60 & 43.3104 & 70.01 & 40.7761 & 89.88 & 5.85 & 43.3912 & 269.48 & 40.8478 & 98.81 & 5.86 \\
\hline 70 & 45.3212 & 82.78 & 42.7483 & 104.6 & 5.68 & 45.3778 & 81.45 & 42.8263 & 112.48 & 5.62 \\
\hline 80 & 46.8119 & 93.78 & 44.3459 & 113.17 & 5.27 & 46.8533 & 397.10 & 44.4367 & 132.42 & 5.16 \\
\hline
\end{tabular}

- The scalability of the $I A M A T$ with respect to the number of records per group.

The scalability of the IAMAT and the $M D A V$ with regard to the group size 


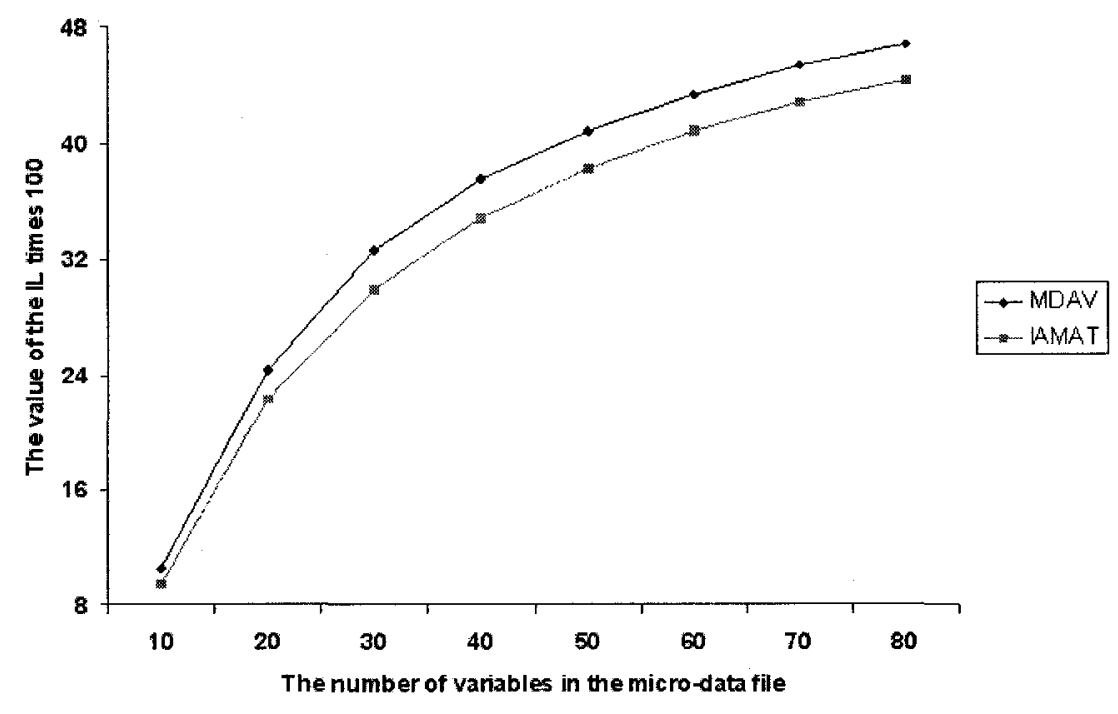

Figure 5.5: The improvement of the IAMAT in reducing the percentage value of the $I L$ as a function of the dimension of the data set using normally distributed data when $k=3$ and $n=10,000$.

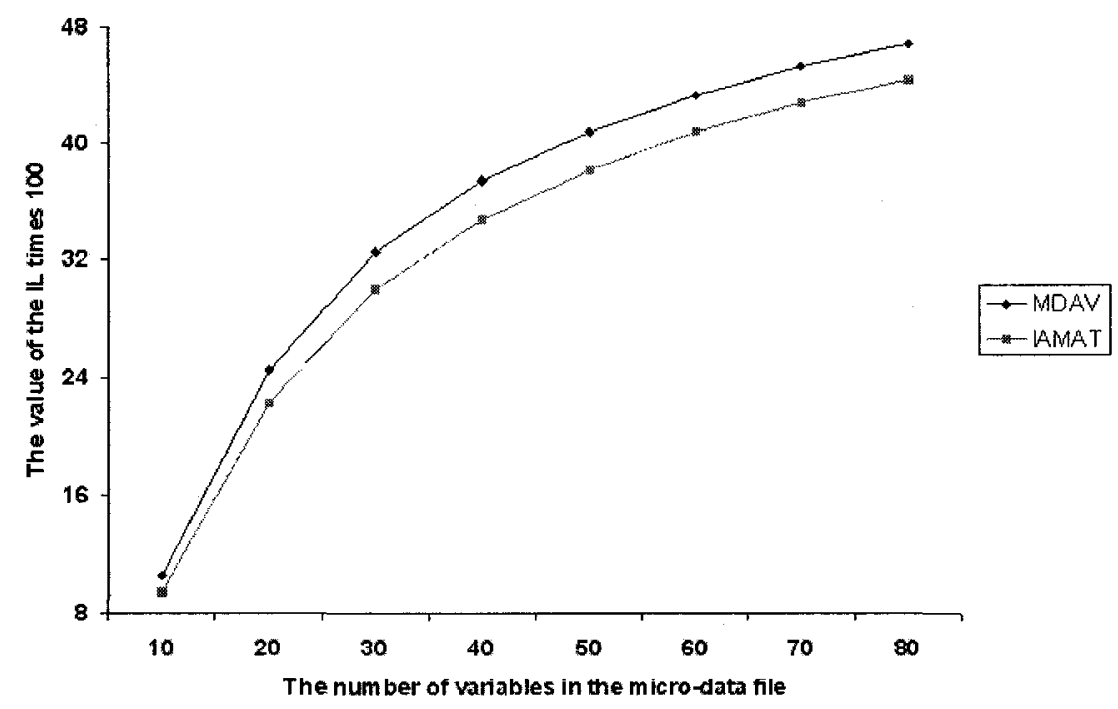

Figure 5.6: The improvement of the IAMAT in reducing the percentage value of the $I L$ as a function of the dimension of the data set using uniformly distributed data when $k=3$ and $n=10,000$. 


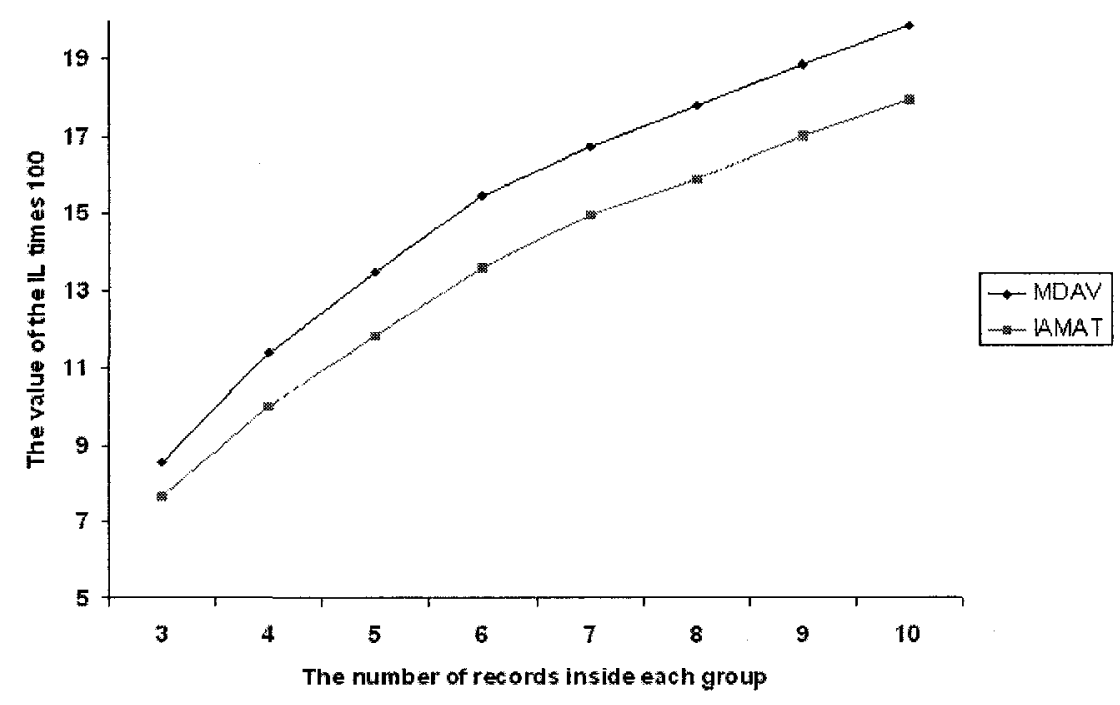

Figure 5.7: The improvement of the IAMAT in reducing the percentage value of the $I L$ as a function of the number of records per group using normally distributed data when $n=10,000$ and $d=10$.

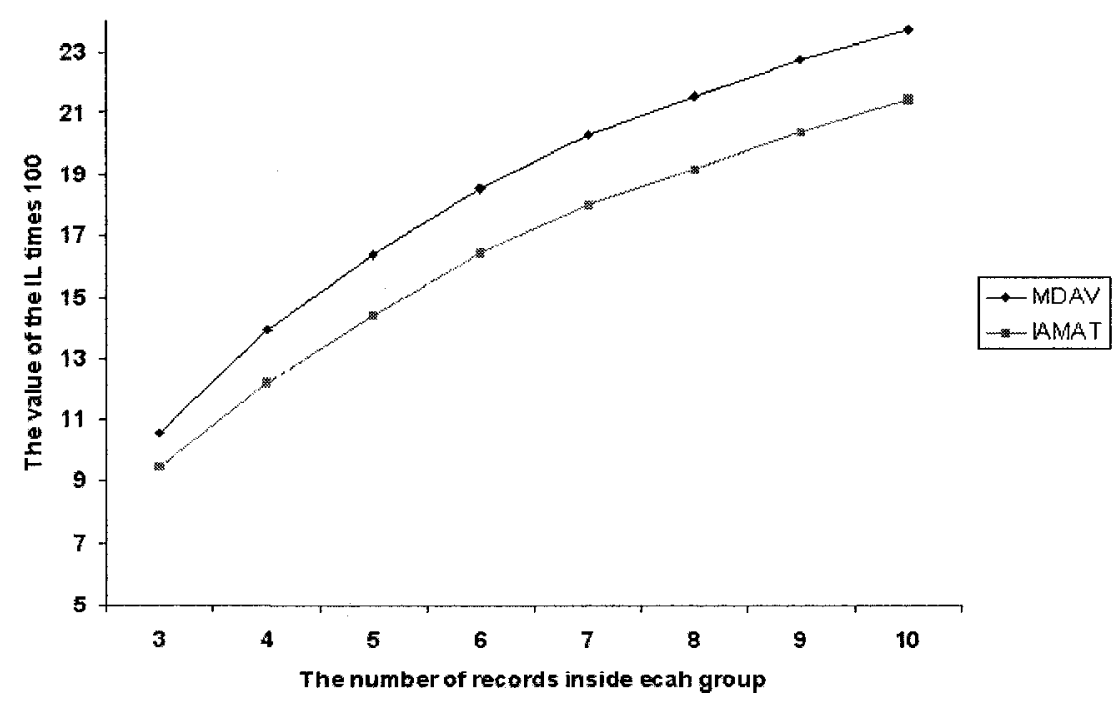

Figure 5.8: The improvement of the IAMAT in reducing the percentage value of the $I L$ as a function of the number of records per group using uniformly distributed data when $n=10,000$ and $d=10$. 
has also been studied for both the uniform and the normal distributions, where the group size ranged from 3 to 10 , and for the cardinality of the data set being 10,000 records, with a dimensionality of 10 variables. Table 5.5 shows the percentage of improvement in reducing the value of the $I L$. This reduction reached $12.74 \%$ for the normal distribution when the group size was 5 , and reached $12.31 \%$ for the uniform distribution when the group size was 4 . Figures 5.7 and 5.8 show that both schemes possess similar trends for the $I L$ as a function of the group size, and that the value of the $I L$ is proportional to the number of records per group. This is, again, a fair observation, because having many records in the group tends to minimize the within-group similarity, and to simultaneously maximize the similarity between the groups. This also tends to increase the value of the $I L$.

Table 5.5: Comparison of the percentage of the $I L$ and the computation time between the $M D A V$ and the $I A M A T$ on simulated data involving uniform and normal distributions. The results demonstrate the scalability of the $I A M A T$ with respect to the number of records per group.

\begin{tabular}{|c|c|c|c|c|c|c|c|c|c|c|}
\hline \multirow{3}{*}{$\begin{array}{c}\mathrm{k} \\
\text { value }\end{array}$} & \multicolumn{5}{|c|}{ Normal Distribution } & \multicolumn{5}{|c|}{ Uniform Distribution } \\
\hline & \multicolumn{2}{|c|}{$M D A V$} & \multicolumn{2}{|c|}{ IAMAT } & \multirow{2}{*}{ improv } & \multicolumn{2}{|c|}{$M \overline{D A V}$} & \multicolumn{2}{|c|}{ IAMAT } & \multirow{2}{*}{ improv } \\
\hline & IL & Time & IL & Time & & IL & Time & $\mathrm{IL}$ & Time & \\
\hline 3 & 10.5383 & 13.5 & 9.4821 & $\mid 32.96$ & 10.02 & 10.543 & 13.7 & 9.4400 & \begin{tabular}{|c|}
33.87 \\
\end{tabular} & 10.47 \\
\hline 4 & 13.9376 & 11.93 & 12.2106 & 28.79 & 12.39 & 13.9202 & 12.24 & 12.2069 & 29.96 & 12.31 \\
\hline 5 & 16.5221 & 11.17 & 14.4167 & 26.67 & 12.74 & 16.4020 & 11.45 & 14.4091 & 29.32 & 12.15 \\
\hline 6 & 18.7797 & 10.68 & 16.4650 & 26.86 & 12.33 & 18.5484 & 10.96 & 16.4152 & 28.78 & 11.50 \\
\hline 7 & 20.3782 & 10.32 & 18.0344 & 25.48 & 11.50 & 20.2887 & 10.56 & 18.0431 & 28.28 & 11.07 \\
\hline 8 & 21.6869 & 10.12 & 19.1562 & 26.67 & 11.67 & 21.5464 & 10.3 & 19.1668 & 26.32 & 11.04 \\
\hline 9 & 22.8931 & 9.87 & 20.4255 & 24.92 & 10.78 & 22.7587 & 10.07 & 20.3952 & 26.02 & 10.39 \\
\hline 10 & 23.8439 & 9.07 & 4969 & 26.06 & 9.84 & 23.6922 & 9.92 & 1.45 & 25.92 & 9.46 \\
\hline
\end{tabular}

\subsection{Conclusions}

In this chapter, we have considered the problem of achieving micro-aggregation in secure statistical databases. The novelty of our method involves enhancing the primitive 
$M A T$ that merely incorporates proximity information. The state-of-the-art $M A T$ recursively reduces the size of the data set by excluding points which were farthest from the centroid and those which were closest to these farthest points. Thus, although the state-of-the-art method was extremely effective, we have argued that it uses only the proximity information, and ignores the mutual Interaction between the records. In this chapter, we have proved that inter-record relationships can be quantified in terms of two entities, namely their "Association" and "Interaction" that can be measured by invoking transitive-closure like operations, and by mapping the problem into a neural setting using the $A C N N$. By repeatedly invoking the inter-record Associations and Interactions, we have shown that the records can be grouped into sizes of cardinality " $k$ ". Our experimental results, which were done on artificial data and on the benchmark data sets for real life data, demonstrate that the newly proposed method is superior to the state-of-the-art by as much as $13 \%$. Thus, we believe that our strategy leads to a very promising tool for solving the $M A P$.

By defining a score, $S I$, as a composite measure involving the $I L$ and $D R$, we see that proposed strategy also obtains a minimum score value when compared to the $M D A V$ method. This indicates that the IAMAT technique is probably the best $M A T$ not only from the $I L$ perspective, but also from the viewpoint of a measure which is as a combination of the $I L$ and $D R$. 


\section{Chapter 6}

\section{Utilizing Dependence-Based Information to Enhance MATs}

\subsection{Introduction}

Although Artificial Intelligent $(A I)$ techniques have been used in various applications, their use in maintaining security in $S D B s$ has not been reported. This chapter ${ }^{1}$ presents results, that to the best of our knowledge is pioneering, by which concepts from causal networks are used to secure SDBs. We consider the MAP in secure SDBs which involves partitioning a set of individual records in a micro-data file into a number of mutually exclusive and exhaustive groups. This problem, which seeks for the best partition of the micro-data file, is known to be NP-hard, and has been tackled using many heuristic solutions. In this chapter, we would like to demonstrate that in the process of developing MATs, it is expedient to incorporate AI-based

\footnotetext{
${ }^{1}$ A preliminary version of some of the results from this chapter appear in the Proceedings of ACISP'08, the Thirteenth Australasian Conference on Information Security and Privacy, in Wollongong, Australia, in July 2008 [108], and in the Proceeding of Al'08, Twenty-First Australasian Joint Conference on Artificial Intelligence, in Auckland, New Zealand in December 2008 [106]. The journal version of these results is currently under review.
} 
causal information about the dependence between the random variables in the microdata file. This can be achieved by pre-processing the micro-data before invoking any $M A T$, in order to extract the useful dependence information from the joint probability distribution of the variables in the micro-data file, and then accomplishing the microaggregation on the "maximally independent" variables. Our results, on real-life and artificial-life data sets, show that including such information will enhance the process of determining how many variables are to be used, and which of them should be used in the micro-aggregation process.

The structure of this chapter is as follows: In Section 6.2 describes how we can enhance micro-aggregation by incorporating dependence, and this is done both informally and algorithmically. Then, in Section 6.3, we present the results of experiments we have carried out for synthetic and real data sets. The chapter finishes in Section 6.4 with some conclusions.

\subsubsection{Contribution of the Chapter}

Central to the study of $S S D S$ are a family of algorithms classified in the literature as being $M A T s$. Apart from being fast and efficient, they are also intuitively appealing because they are akin to the family of clustering methods. This chapter considers how such methods can be enhanced, both with regard to "accuracy" and efficiency, by learning, and thereafter incorporating the information that relates to the dependence between the random variables being analyzed. In all brevity, we are not aware of any other reported method which specifically incorporates such dependence-type information to optimize an $M A T$, or for that matter, to optimize a method which controls the $I L$ and the $D R$ in $S S D s$.

Understanding the presence and structure of dependency between a set of random variables is a fundamental problem in the design and analysis of many types of systems including filtering, pattern recognition etc. As far as we know its application in SDC has been minimal. Utilizing this information is the goal of this chapter. Typically, in 
modern day systems, the data protector has been able to choose the technique and set its parameters without a thorough understanding of the characteristics of the microdata file, and the stochastic dependence of the variables. Although gleaning this information could be particularly difficult and even time-consuming, our hypothesis is that this information is central to the micro-data file, especially when working in a high dimensional space.

In general, the result of the multi-variate $M A T s$ depends on the number of variables used in the micro-aggregation process. In other words, deciding on the number of variables to be taken into account, and on the identity of the variables to be microaggregated, is far from trivial. Domingo-Ferrer and Torra have reported in [54] that multi-variate micro-aggregation on unprojected data taking two or three variables at a time (rather than incorporating the information in all the variables) offers the best trade-off between $I L$ and $D R$. The unanswered question is that of inferring which variables should be used in this process. We believe that a solution to this puzzle lies in the inter-variable "dependence" information.

The authors of [120] have emphasized that the decision about which variables are to be chosen has to be gleaned from a priori "knowledge about the characteristics of each variable from the experts". While this is a feasible approach, we argue that it is subjective, and that a formal objective method is desirable. Indeed, what will happen if the researcher encounters a new project for which there is no prior knowledge? Or how we will proceed if an expert for a specific data domain is not available? Our aim is to minimize the necessity to depend on a human expert, but rather to have the ability to study and estimate the characteristics of each variable objectively. Thus, we seek a systematic process by which we can choose the desired variables automatically and, thereafter, micro-aggregate the file.

This chapter involves $M A T s$, but rather from a perspective different than the ones that have been considered in the literature. We propose a scheme by which we can avoid using the information in all the dimensions (for example, in computing the distance between two records etc.). Furthermore, neither will we resort to projecting 
the micro-data file onto a single axis, nor will we attempt to micro-aggregate it using any specific sorting method [31, 32, 33, 34, 89, 91, 92, 115]. The main contribution of this chapter is to extract useful information from the joint probability distribution of the variables in the file to be micro-aggregated. Then, rather than use all the variables in the micro-data file, we propose to only process the "maximally independent" variables in the subsequent multi-variate micro-aggregation. Indeed, we propose to use such a method as a pre-processing step before any $M A T$ is invoked, and to test the effect of using such a dependency analysis on the micro-aggregation process so as to reduce the computational time, and $I L$.

\subsection{Enhancing Micro-Aggregation with Dependence $(E M A D)$}

It is well-known that the result of the multi-variate MATs depends on the number and the identity of the variables used in the micro-aggregation process. Since multi-variate micro-aggregation using two or three variables at a time offers the best trade-off between the $I L$ and the $D R$ [54], the question we intend to resolve involves understanding why we have to maintain and use vast dimension-dependent resources in the clustering phase in order to compute the distance between the micro-records. We shall also study how we can minimize the computation time needed to evaluate the distance between a single micro-data record and the mean of the group it belongs to. This computation involves evaluating

$$
D(X, Y)=\sqrt{\sum_{i=1}^{d}\left(x_{i}-y_{i}\right)^{2}}
$$

where $X$ and $Y$ are two multi-variate data vectors with their components being $\left\{x_{i}\right\}$ and $\left\{y_{i}\right\}$ respectively, and $d$ represents the dimension of the space. 
We consider the problem of determining the dependencies between the different variables within a micro-data file, and then combining the latter with the MAT in such a way as to reduce the overall required computational time, and/or reduce the corresponding $I L$.

The primary goal of any $M A T$ is to reduce the loss in the data utility by choosing the most suitable sub-set of variables with size equal to three [54] prior to invoking the multi-variate micro-aggregate. Theoretically, to know the best sub-set of variables that has to be used in order to obtain the minimum value of the $I L$, we have to consider all different possibilities of combinations, namely the $\left(\begin{array}{l}S \\ C\end{array}\right)=\frac{S !}{C !(S-C) !}$ combinations, where $S$ is the number of variables in the original micro-data file, and $C$ is the number of chosen variables which are used in projecting and micro-aggregating the data file.

We propose that the key idea in choosing a sub-set of the variables by avoiding the combinatorial solution, should be based on the dependence model of the micro-data file. If the variables are highly-correlated, then using any one of them will somehow reflect the stochastic nature of the others. If we, thus, incorporate this logic into our consideration, we believe that we can reduce the number of variables which will be used to measure either the distance between the micro-unit and the mean of the group it belongs to, or the distance between the micro-units themselves. Thus, in turn, this will reduce the dimensionality of the space to $d^{\prime}<d$. The new distance that will thus be computed will be:

$$
D^{\prime}(X, Y)=\sqrt{\sum_{i=1}^{d^{\prime}}\left(x_{i}-y_{i}\right)^{2}} \quad \text { where } d^{\prime}<d
$$

The reader should observe that our goal is quite distinct from the reported methods of projecting the multi-dimensional space onto a single axis using a particular variable, the sum $z$-scores scheme, or a principal component analysis. The reduction in the dimensionality is not done randomly. Rather it is to be done based on a formal criterion. Our aim is to micro-aggregate the multi-dimensional vector by maximally 
using the information in the "almost-independent" variables, and we plan to do this by finding the best dependence tree. We believe that we can achieve this by evaluating the dependence between the variables in the micro-data file by using either the method due to Chow and Liu [17] or the method due to Valiveti and Oommen $[135,136]$.

We formalize these concepts below. The joint probability distribution of the random vector $\mathbf{V}=\left[V_{1}, V_{2}, \ldots, V_{d}\right]^{T}$ in terms of conditional probabilities is given as

$$
P(\mathbf{V})=P\left(V_{1}\right) P\left(V_{2} \mid V_{1}\right) P\left(V_{3} \mid V_{1}, V_{2}\right) \ldots P\left(V_{d} \mid V_{1}, V_{2}, \ldots, V_{d-1}\right)
$$

where each $V_{i}$ is a random variable.

It is obvious, from the above expression, that each variable is conditioned on an increasing number of other variables. Therefore, estimating the $k^{\text {th }}$ term of this equation requires maintaining the estimates of all the $k^{\text {th }}$ order marginals. Clearly, it is impractical to gather the estimates for the joint density function $P(\mathrm{~V})$ for all the different values which $V$ could assume. We, therefore, simplify the dependency model by restricting ourselves to the lower-order marginals, using the approximation which ignores the conditioning on multiple variables, and retaining only dependencies on at most a single variable at a time. This leads us to the following [135]:

$$
P_{a}(\mathbf{V})=\prod_{i=1}^{d} \operatorname{Pr}\left(V_{i} \mid V_{j(i)}\right)
$$

where $P_{a}(\mathbf{V})$ is the approximated form of $P(\mathbf{V})$, and $V_{i}$ is conditioned on $V_{j(i)}$ for $0 \leq j(i)<i$.

The dependence of the variables can be represented as a graph $\mathbf{G}=(\mathbf{V}, \mathbf{E}, \mathbf{W})$ where $\mathbf{V}=\left\{V_{1}, V_{2}, \ldots, V_{d}\right\}$ is a finite set of vertices, which represents the set of random variables in the micro-data file with $d$ dimensions, $\mathbf{E}$ is a finite set of edges $\left\{\left\langle V_{i}, V_{j}\right\rangle\right\}$, where $\left\langle V_{i}, V_{j}\right\rangle$ represents an edge between the vertices $V_{i}$ and $V_{j}$. Finally, $\mathbf{W}=\left\{w_{i, j}\right\}$ is a finite set of weights, where $w_{i, j}$ is the weight assigned to the edge 
$\left\langle V_{i}, V_{j}\right\rangle$ in the graph. The values of these weights can be calculated based on a number of measures, as will be explained presently.

In $\mathbf{G}$, the edge between any two nodes represents the fact that these variables are statistically dependent [17]. In such a case, the weight, $w_{i, j}$, can be assigned to the edge as being equal to the Expected Mutual Information Measure (EMIM) metric between them. Generally speaking, the EMIM metric between two variables, given by $I^{*}\left(V_{i}, V j\right)$ for discrete distributions, has the form:

$$
I^{*}\left(V_{i}, V_{j}\right)=\sum_{v_{i}, v_{j}} \operatorname{Pr}\left(v_{i}, v_{j}\right) \log \frac{\operatorname{Pr}\left(v_{i}, v_{j}\right)}{\operatorname{Pr}\left(v_{i}\right) \operatorname{Pr}\left(v_{j}\right)}
$$

where the summation above is done over all values of $v_{i}$ and $v_{j}$ which $V_{i}$ and $V_{j}$ can assume.

Observe that any edge, say $\left\langle V_{i}, V_{j}\right\rangle$ with the edge weight $I^{*}\left(V_{i}, V_{j}\right)$ represents the fact that $V_{i}$ is stochastically dependent on $V_{j}$, or that $V_{j}$ is stochastically dependent on $V_{i}$. Although, in the worst case, any variable pair could be dependent, the model expressed by Eq.(6.4) imposes a tree-like dependence. It is easy to see that this graph includes a large number of trees (actually, an $\bigcirc\left(d^{(d-2)}\right)$ of such spanning trees). Each of these trees represents a unique approximated form for the density function $P(\mathbf{V})$. Chow and Liu proved that searching for the best "dependence tree" is exactly equivalent to searching for the Maximum Spanning $\operatorname{Tree}^{2}(M S T)$ of the graph [17]. Further, since the probabilities that are required for computing the edge weights are not known a priori, Valiveti and Oommen showed that this could be achieved by estimating them in a maximum likelihood manner $[135,136]$. They showed that the Maximum Likelihood $(M L)$ estimate for the best dependence tree, can be obtained

\footnotetext{
${ }^{2}$ Two generic greedy algorithms can be used to solve the Minimum Spanning Tree problem, namely, the so-called Kruskal and the so-called Prim algorithms. Both of them run in time $O(E \lg V)$ by using ordinary binary heaps $[18,118,125]$. Since we are attempting to compute the Maximum Spanning tree, it is obvious that we have to order the edges in a decreasing order (as in Kruskal) or to extract the maximum edges weight (as in Prim). We have used the Kruskal algorithm in our experiments.
} 
by computing the $M S T$ of the graph, where the edge weights are computed using the EMIM of the estimated probabilities, as shown in Figure 6.1.

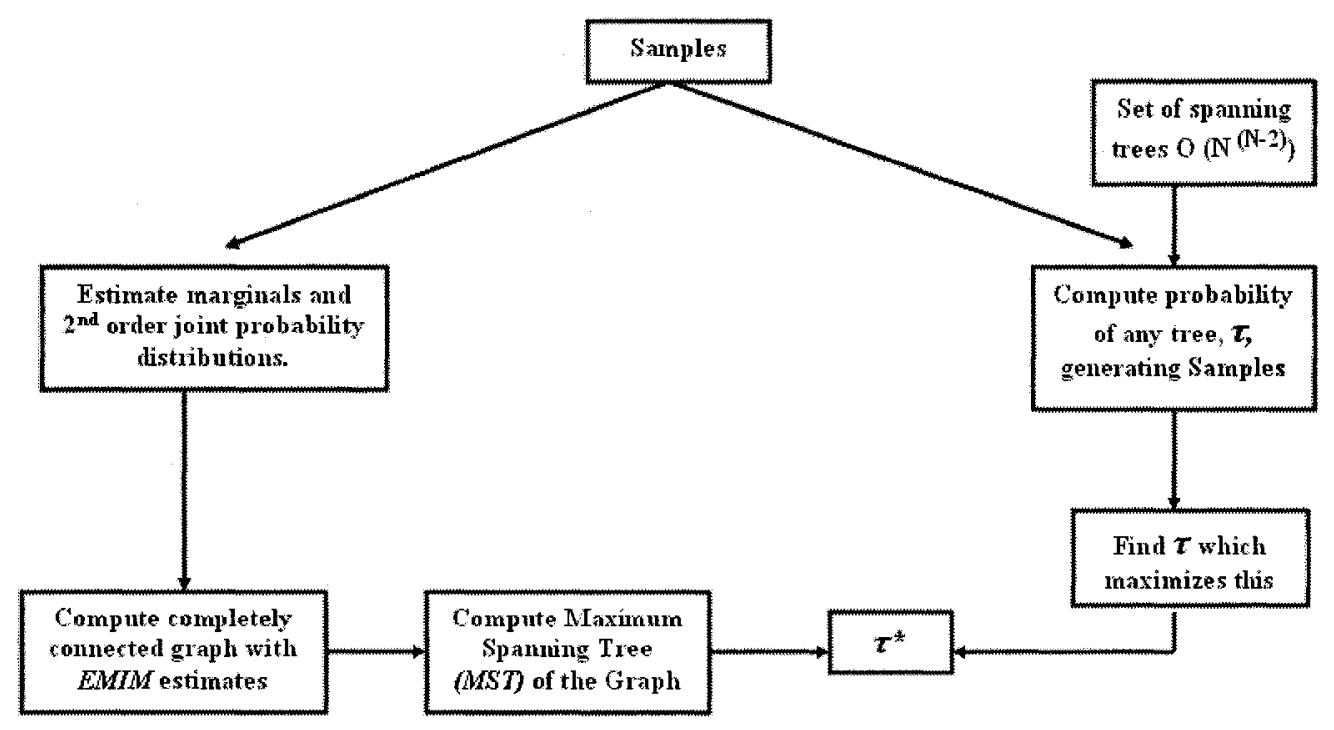

Figure 6.1: Equivalent procedures for finding the Maximum Likelihood Estimate of the tree-based dependence from the samples.

It is worth mentioning that this solution is truly both elegant and efficient. A rigorous $M L$ solution to obtaining the best tree would involve computing it from the set of all possible spanning trees, which (at the enumeration level itself) is a combinatorially explosive problem. To solve the $M L$ problem in a formal manner, one has to first obtain the set of all the graph's spanning trees, and then determine the tree which maximizes the likelihood function evaluated in terms of the dependence described by the tree itself. Observe that the solution obtained by solving for the $M S T$ is many orders of magnitude less complex. It involves estimating the probabilities (and not the structure) of the Binomial (multinomial) distributions using a $M L$ estimate, and then merely computing the $M S T$. The fact that these two processes lead to the same estimate (as shown in Figure 6.1) is far from trivial to prove, but is 
indeed, true.

It should be mentioned here that the weights of the edges in the graph, $\mathbf{G}$, can be computed using either the EMIM metric or the $\chi^{2}$ metric proposed by Valiveti and Oommen [135]. The latter, $I_{\chi}\left(V_{i}, V_{j}\right)$, is an alternative measure that quantifies the dependence information between pairs of random variables, and is computed by:

$$
I_{\chi}\left(V_{i}, V_{j}\right)=\sum_{v_{i}, v_{j}} \frac{\left(\operatorname{Pr}\left(v_{i}, v_{j}\right)-P\left(v_{i}\right) P\left(v_{j}\right)\right)^{2}}{P\left(v_{i}\right) P\left(v_{j}\right)} .
$$

$I_{\chi}$ has the following desirable characteristics relevant to capturing dependence information:

$$
\left\{\begin{array}{cl}
I_{\chi}\left(V_{i}, V_{j}\right)=0 & \text { iff } P\left(v_{i}, v_{j}\right)=P\left(v_{i}\right) P\left(v_{j}\right) \\
I_{\chi}\left(V_{i}, V_{j}\right)>0 & \text { otherwise. }
\end{array}\right.
$$

It turns out that for binary and normally distributed random variables, the $I_{\chi}$ metric is exactly equivalent to the $I^{*}$ metric in finding the dependence tree $[135$, 136]. But, when the underlying dependence is not actually based on a tree structure, both of them estimate the best dependence tree corresponding to their representative measures. Valiveti and Oommen showed the interesting feature that although their estimation for the best dependence tree does not, always match, the total weights are almost always identical.

By way of example, consider a micro-data file which incorporates 6 variables (as in Figure 6.2) and thousands of records. Let us assume that we intend to microaggregate this file using any $M A T$, for example, the $M D A V$ method. In such a case, the prior art will process all the six variables to quantify the relevant distances during the clustering stage. We could choose a sub-set of size three to be used in the micro-aggregation process. In general, we will have to go through the 20 different combinations of size three in order to attain the minimum value of the $I L$. However, if we are able to discover any existing inter-variable dependencies, this could render 


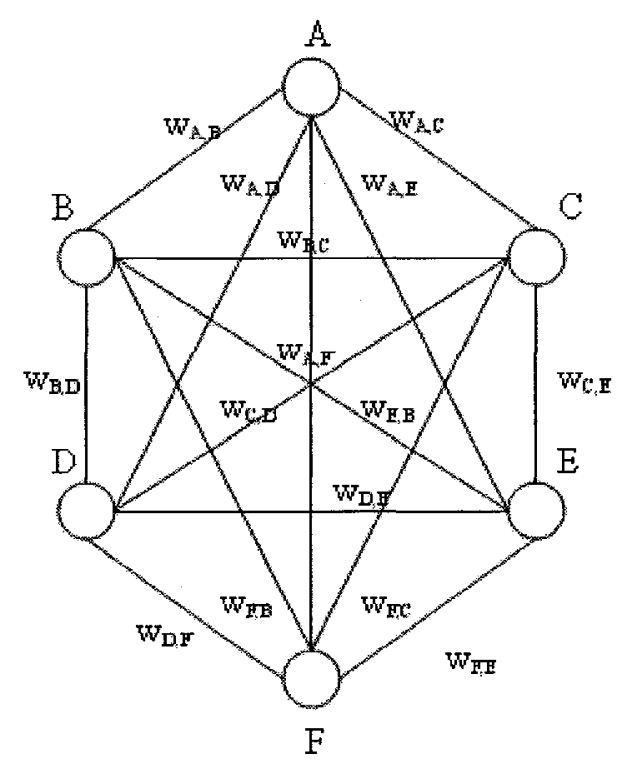

Figure 6.2: The fully-connected undirected graph represents the dependence between six random variables.

the problem simpler. Let us assume that we compute the $E M I M$-based edge weights for all pairs of nodes, and create the fully-connected undirected graph $G$, as in Figure 6.2. By using the strategy alluded to above, we obtain a tree as in Figure 6.3.a, which shows the case when the $M S T$ leads to the $M L$ condition that the variables $B, C$, and $D$ depend on the variable $A$, and that variables $E$ and $F$ depend on variable $D$.

Since these dependent variables are maximally-correlated to the variable that they depend on, we propose to use the vertices that have the maximum number of In/Out edges in the graph to micro-aggregate the micro-file. We believe that the nodes which possess this property are the best candidates to reflect the characteristics of the entire multi-variate data set because they connect to the maximum number of nodes that statistically depend on it, as argued in Conjecture 1.

Conjecture 1. Micro-aggregating the micro-data file can be best achieved if the nodes which possess the maximum number of In/Out edges in the tree obtained as the MST of the underlying undirected connected graph $G$, are used as the input to solve the 
MAT.

\section{Rational for Conjecture}

The existence of an edge between two nodes in the connected undirected graph signifies that these two nodes are statistically correlated to each other, and that a variation of one of these variable is reflected by a corresponding change in the other. Thus, the variables which are connected to each other via edges in the skeletal tree represent nodes which are connected to each other based on the best tree-based dependence, and in turn, reflect the maximal shared characteristics within the variables of the micro-data file. Thus, any node which has a larger number of In/Out edges is one which connects to a larger number of nodes, and is thus capable of individually representing more "other" variables. This implies that the best candidates to be used to represent the other variables in the micro-aggregation are those which have the maximum number of In/Out edges.

In order to invoke this property, we first rank the nodes of the graph based on the number of In/Out edges in a descending order and choose the first $d^{\prime}$ variables, where $d^{\prime}$ is usually determined by the data protector, and is usually equal to 3 or 4 . Thus, for example, based on the above discussion, for the data represented by the variables of Figure 6.3, the micro-aggregation process will be invoked by using two variables instead of using the entire set of six variables in the micro-data file. Figure 6.3(b) shows that the selected sub-set of the variables is $\{A, D\}$, since both of them connect to 3 variables while the other variables in the micro-data file connect to only a single variable. The process outlined above has been formalized in Algorithm 13 which presents an automated way to select a sub-set of the variables to be used in the multi-variate micro-aggregation process. 


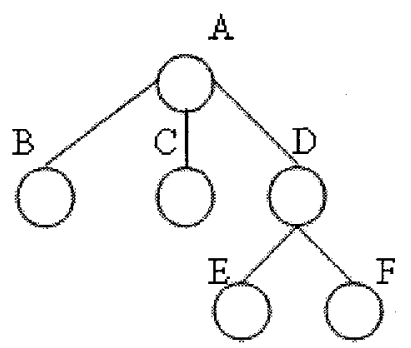

(a)

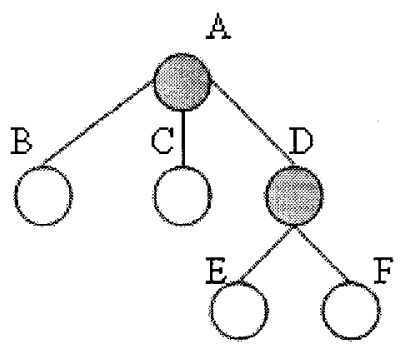

(b)

Figure 6.3: An example of a dependence tree used to micro-aggregate the data file containing 6 variables.

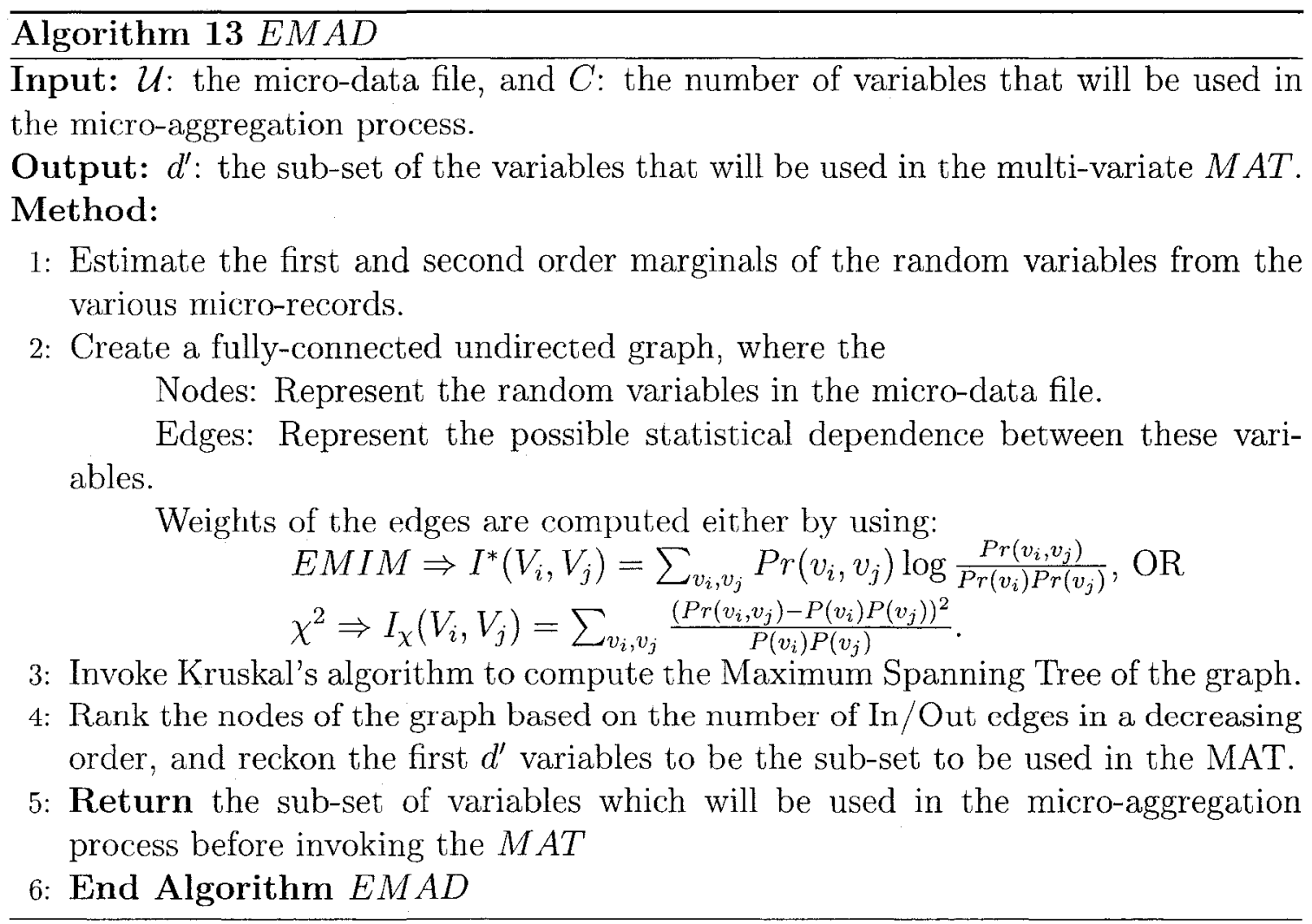




\subsection{Experimental Results}

\subsubsection{Data Sets}

In order to verify the validity of our methodology in projecting the multi-variate data set into a subset of random variables to be used in the micro-aggregation process, two benchmark real-life data sets and three simulated data sets were used in the testing phase. Table 6.1 summarizes the characteristics of each data set by defining its type, dimensionality and cardinality.

Table 6.1: The characteristics of various data sets.

\begin{tabular}{|c|c|c|c|}
\hline Name of the data set & Type & Dimensionality & Cardinality \\
\hline \hline Tarragona & Real & 13 & 834 \\
\hline Census & Real & 13 & 1080 \\
\hline Sim_1 & Simulated & 8 & 5000 \\
\hline Sim_2 & Simulated & 16 & 10,000 \\
\hline Sim_3 & Simulated & 22 & 20,000 \\
\hline
\end{tabular}

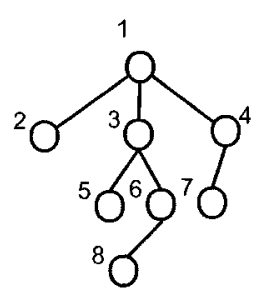

(a) Sim_1

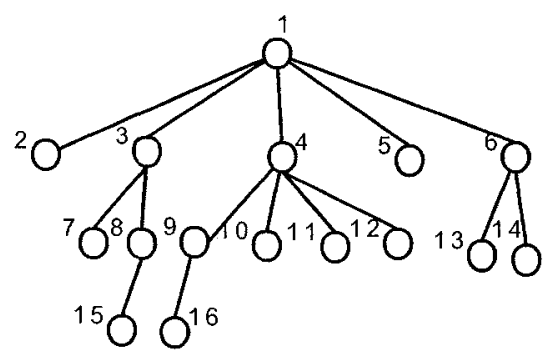

(b) Sim_2

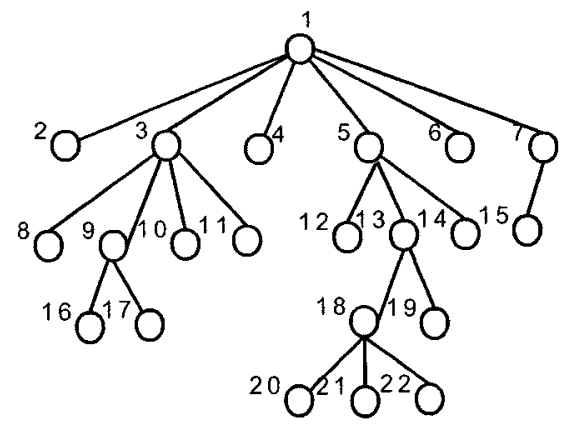

(c) Sim_3

Figure 6.4: The true structures for the simulated data sets

The Tarragona and Census benchmarks are reference data sets used in previous studies for their special statistical properties $[45,53]$. On the other hand, the simulated data were generated or tested for various dimensions of random vectors, as 
follows: First of all, the number of random variables was determined. Thereafter, the "true" structure of the defined dependence tree which imposed the dependence relationships between the variables was selected subjectively, as shown in Figure 6.4. Then the second-order marginal distributions were randomly generated. The procedure by which these were generated was as follows: If we define the entire space of each variable to be between 1 and 1000, this space is sub-divided into a number of subspaces with equal width, say 100 . That means that we limit ourselves to be dealing with 10 events where each event represents a sub-interval of width equal to 100 from the entire domain as follows: $\left\{I_{1}=[1,100], I_{2}=[101,200], \ldots, I_{10}=[901,1000]\right\}$, thus, effectively simulating a multinomial distribution. In the latter, each outcome is a random number belonging to exactly one of the 10 sub-intervals, $I_{j}$, with probability, $P_{j}$, where $j=1,2, \ldots, 10$. If $n_{j}$ represents the number of occurrences of values belonging to $I_{j}$ and $n$ represents the number of independent records, we have

$$
\sum_{i=1}^{10} n_{i}=n, \sum_{i=1}^{10} P_{i}=1
$$

where the probability mass function of the multinomial distribution is

$$
f\left(n_{1}, n_{2}, \ldots, n_{10}\right)=\frac{n^{!}}{n_{1}^{!} n_{2}^{!} \ldots n_{10}^{!}} \prod_{i=1}^{10} P_{i}^{n_{i}}
$$

Observe that prior to assigning the second order marginal distributions for the rest of the tree, we had to also randomly generate 10 different probabilities for the most independent variables (the root variable) when its values belonged to each of the above defined sub-intervals.

To randomly populate the file, we can now randomly assign values to the conditional probability from the joint and marginal distributions as follows: If, as per the assumed tree-based dependence, variable $V_{m}$, depends on variable $V_{n}$, this means we have to define a set of probabilities, $\left\{P_{n m}\right\}$, when the value of $V_{n}$, say $v_{i n}$, belongs 
to any defined sub-interval $I_{j}$ given that the value of variable $V_{m}$, say $v_{i m}$ belongs to any sub-interval $I_{l}$. Thus,

$$
P_{m n}=\operatorname{Pr}\left(v_{i n} \in I_{j} \mid v_{i m} \in I_{l}\right),
$$

where $i$ represents the index of the record in the micro-data file and assumes values in $\{1,2, \ldots, n\}$. The indices $j$ and $l$ represent the indices of the sub-interval where the random variable falls, and which are the result of dividing the entire domain into 10 sub-intervals. Finally, the indices $n$ and $m$ represent the specific dimensions in the micro-data file, and are in the range $\{1, \ldots, d\}, n \neq m$.

The above procedure was implemented for all pairwise combinations of random variables associated with the micro-data file.

\subsubsection{Results}

The experiments conducted were of five categories: In the first set of experiments the intention was primarily focused on testing whether the best dependence tree can be learned (or rather, inferred) from the continuous micro-data file, and if it sufficiently reflected the dependence model. In the second set of experiments, the goal was to investigate whether the algorithm is able to infer the dependence model between the random variables when additional information about the dependency between the sibling in the tree is available. In the third set of experiments, the goal was primarily to validate our strategy for determining the subset of variables (from the entire set of variables) to micro-aggregate the micro-data file, and to study its effect on the value of the $I L$. The fourth set of experiments was designed to determine the most suitable metric to calculate the edge weights of the fully-connected graph so as to minimize the required computation time and maximize the accuracy of estimating the dependence model and its effect on the value of the $I L$. Finally, since we are working with continuous vectors, the last set of experiments focused on understanding the effect 
of assuming normality (i.e, the relevance of the Central Limit Theorem [61]) on the data set in calculating the edges weights.

\section{- Experiment Sets 1:}

The first set of experiments was done on two types of data sets: Simulated data sets with a known structure of the best dependence tree which is to be inferred by the learning algorithm, and the real data sets possessing an unknown dependence model between the variables. It is worth mentioning that we could not approximate the dependence information of the multi-variate data set in its current form due to the inaccurate estimation for the joint and marginal probability distributions for continuous variables. This is a consequence of having a large domain space with only few records (sometimes only one or two) for each region of the corresponding random variable. Consequently, most of the estimated marginal and joint probability values were close to zero. Clearly, in these cases, the estimated probabilities will not reflect the actual dependence relationship between any corresponding variables.

In order to overcome this challenging problem that prevents us from utilizing the dependence information, we were forced to reduce the domain space by categorizing the micro-data file as follows: We first scanned the micro-data file to specify the domain space of each variable in the file, and then divided it into a number of sub-intervals sharing the same width. After that, we achieved a categorization phase by replacing the values belonging to a certain sub-interval in each variable by the corresponding category/code. For example, in the case of the simulated data sets, all the variables shared the same domain space between 1 and 1000, which was divided into 10 subintervals, as explained earlier. Consequently, all values belong to the $[1,100]$ interval were replaced by 1 , all values belong to the $[101,200]$ interval were replaced by 2 and so on. The above procedure was repeated for all the variables so as to generate the categorical micro-data file.

From the above discussion, it is clearly shown that "width" parameter plays a predominant role in controlling the degree of smoothing and estimating the best 
dependence tree. Our experiments indicated that assigning a suitable value to the width parameter guaranteed the convergence of the $M S T$ to the true underlying (unknown) structure of the best dependence tree. The most important point that one has to be aware of in a practical scenario is that a larger value for the width parameter implies a lower variance and a higher bias, because we are essentially assuming a constant value within the sub-interval. Generally speaking, the value of the width parameter should be large enough to generate a sufficient number of sub-intervals from the defined domain space to guarantee a satisfactory level of smoothing. The actual value used is specified in the respective experimental results.

Consider the tree structure given by Sim_1, Sim_2, and Sim_3 as given in Figure 6.4. Approximating the dependence information of the simulated data sets based on the structure of the MST obtained using the EMIM metric succeeded in locating the real structure when the width parameter was set to the values 50, 100, and 150 for Sim_1, 70, 100, and 120 for Sim_2, and 90, 100 and 110 for Sim_3. Figure 6.5 shows the edge weights and the value of $I_{x}$ for each simulated data set when the value of width was equal to 100. Figures 6.6, 6.7 and 6.8 show different snapshots of the convergence to the final structure of the dependence model for various sample sizes for Sim_1, Sim_2 and Sim_3, respectively, when the value of the width parameter was set to 100 .

Approximating the dependence information for the real data sets was a little more "tricky", because of the unknown structure for the best dependence tree. Changing the value of the width parameter has an effect on the structure of the best dependence tree to which the algorithm converged. Figures 6.9 and 6.10 clearly show different structures for the best dependence tree by changing the value of the width for the Tarragona and Census data sets, respectively.

\section{- Experiment Sets 2:}

The second set of experiments involves the so-called Sibling-related Model. The aim here was to see if the algorithms possessed the ability to infer the structure of the dependence model between the random variables if additional information 


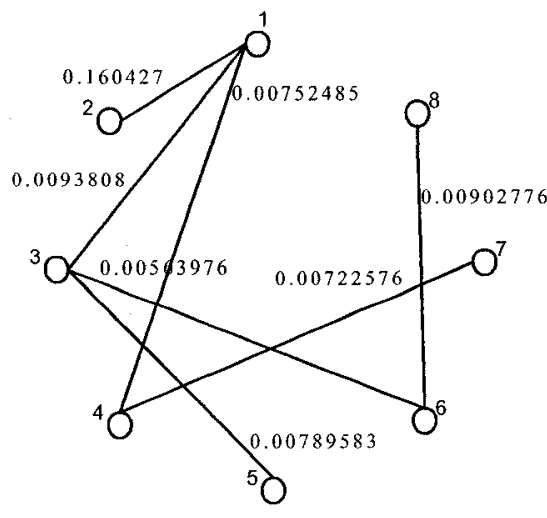

The Total Wcight $=0.207122$

(a) $\operatorname{Sim} 1$

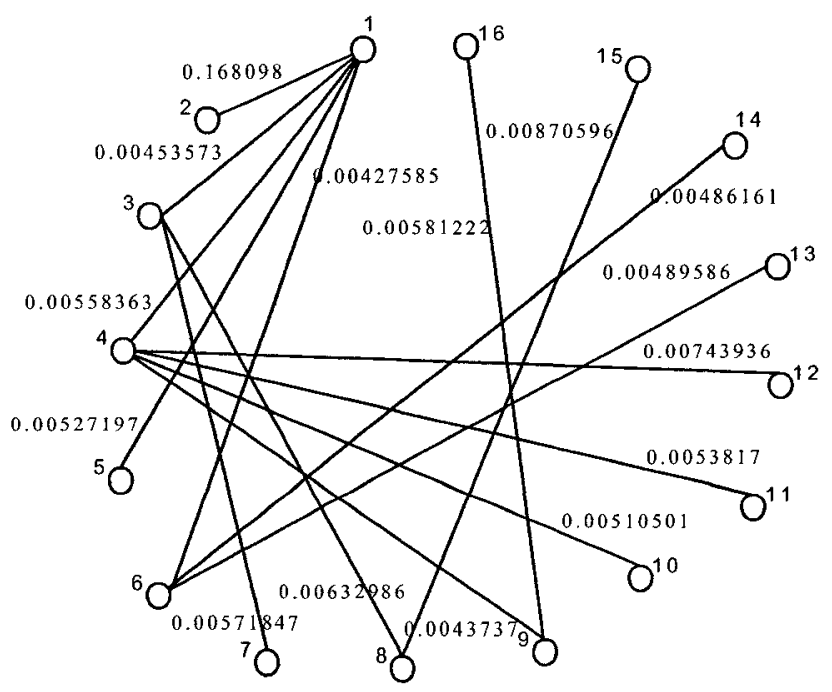

The Total Weight $=0.246389$

(b) Sim_2

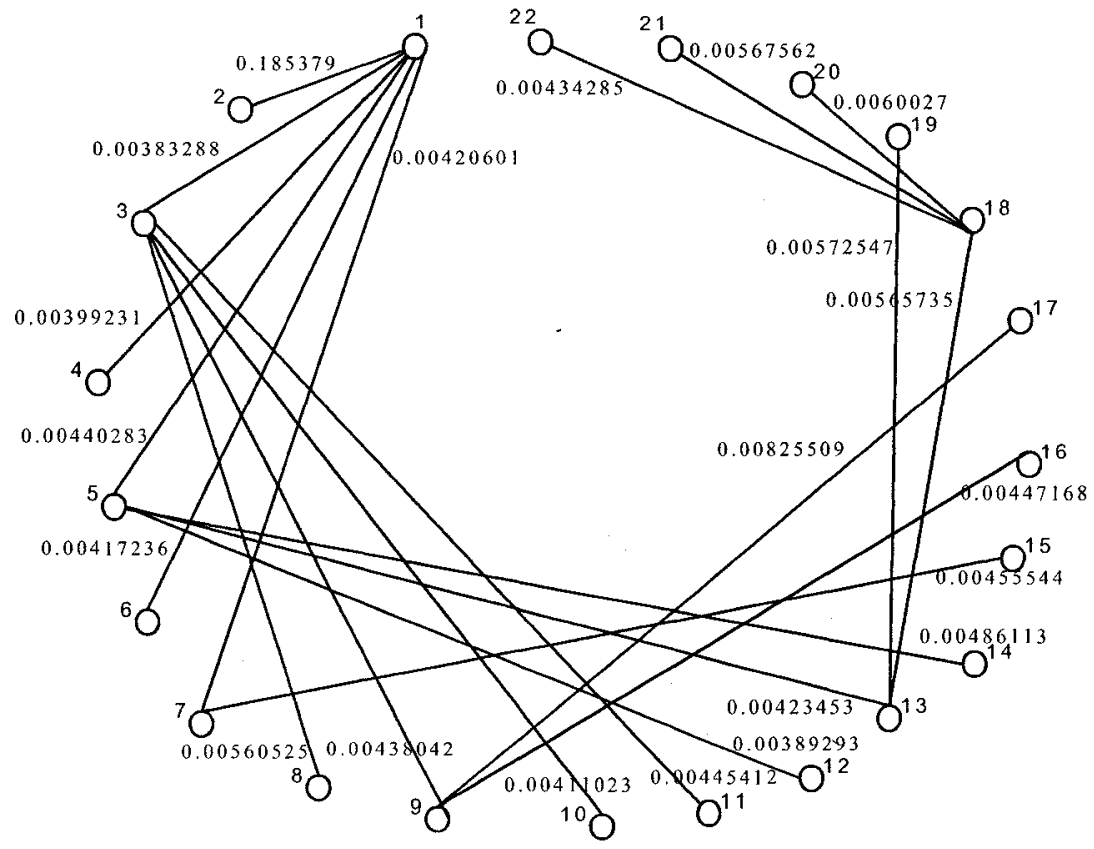

The Total Weight $=0.282211$

(c) Sim_3

Figure 6.5: The best dependence tree for the simulated data sets obtained by using the EMIM metric. 


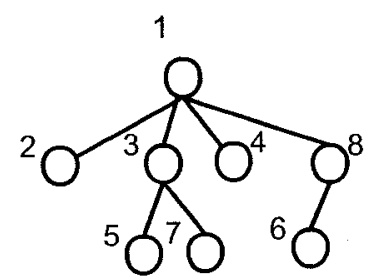

(a) 1000

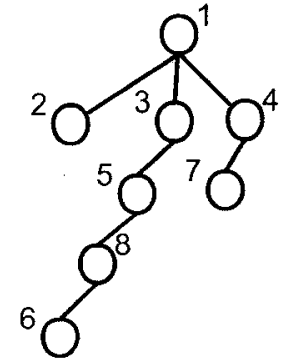

(b) 2500

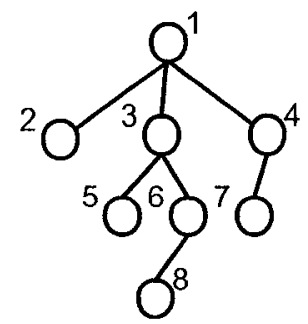

(c) 4000

Figure 6.6: The "inferred" dependence tree for the Sim_1 binary data set as the number of samples increases. The width parameter was set to 100 .

about the dependency between the siblings in the tree is available. The results that we have obtained are quite amazing.

To be more specific, we consider the possibility that after the structure of the underlying tree is determined, the probability values between the siblings in the structural tree are related. For example, thus, if a particular node had index $i$ and its children were nodes $j$ and $k$, the probabilities that could be independently set were:

$$
\begin{aligned}
& \operatorname{Pr}\left[x_{j}=0 \mid x_{i}=0\right] \\
& \operatorname{Pr}\left[x_{j}=0 \mid x_{i}=1\right]
\end{aligned}
$$

Since the probabilities of the siblings were thus determined, the values of $\operatorname{Pr}\left[x_{k}=\right.$ $\left.0 \mid x_{i}=0\right]$ and $\operatorname{Pr}\left[x_{k}=0 \mid x_{i}=1\right]$ were then set to be $1-\operatorname{Pr}\left[x_{j}=0 \mid x_{i}=0\right]$ and $1-\operatorname{Pr}\left[x_{j}=0 \mid x_{i}=1\right]$ respectively. Further, observe that a result of these assignments, the probabilities, $\operatorname{Pr}\left[x_{j}=1 \mid x_{i}=0\right], \operatorname{Pr}\left[x_{j}=1 \mid x_{i}=1\right]$, $\operatorname{Pr}\left[x_{k}=1 \mid x_{i}=0\right]$ and $\operatorname{Pr}\left[x_{k}=1 \mid x_{i}=1\right]$ were automatically assigned, since the sum of these quantities and the values of their counterparts, is unity.

The question we were interested in investigating was to see if our strategy for 


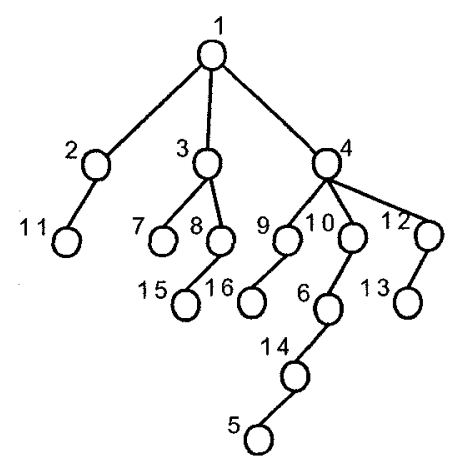

(a) 1000

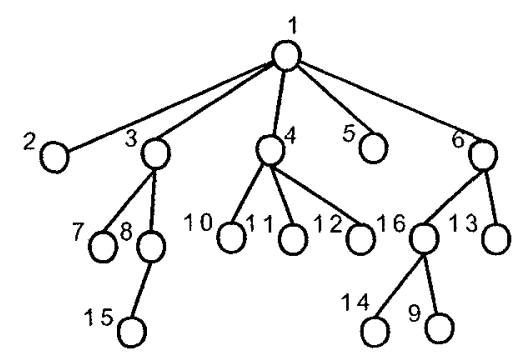

(b) 2500

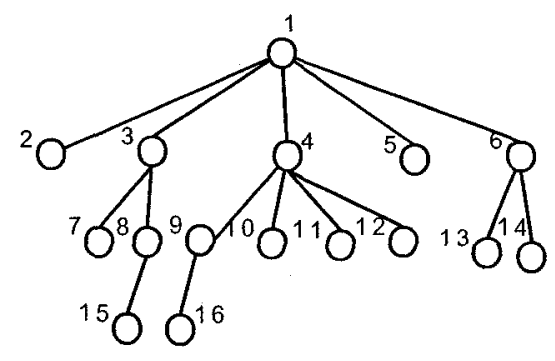

(c) 4000

Figure 6.7: The "inferred" dependence tree for the Sim_2 binary data set as the number of samples increases. The width parameter was set to 100 . 


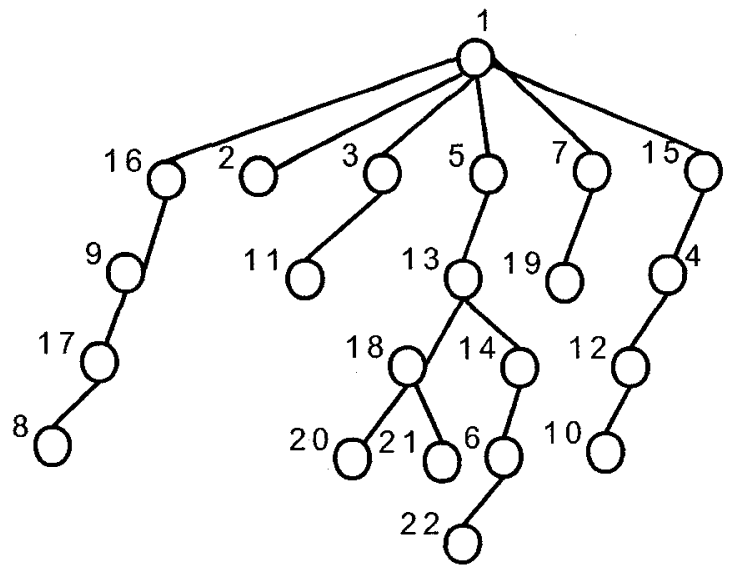

(a) 1000

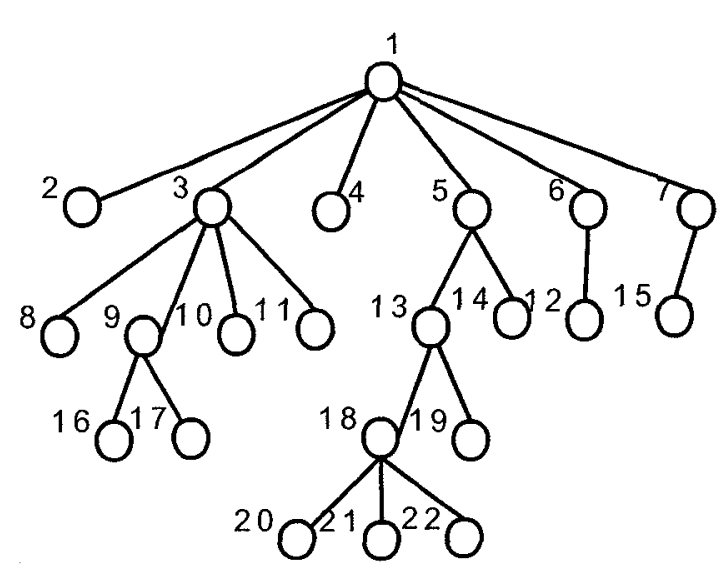

(b) 2500

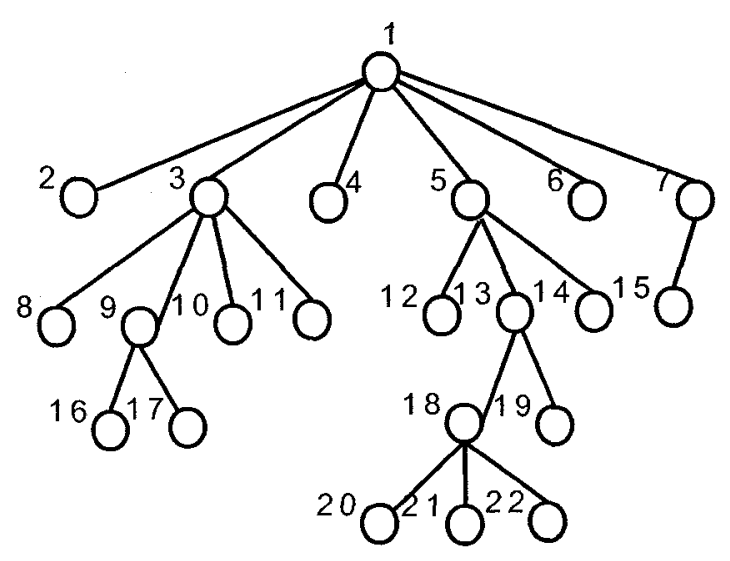

(c) 4000

Figure 6.8: The "inferred" dependence tree for the Sim_3 binary data set as the number of samples increases. The width parameter was set to 100 . 


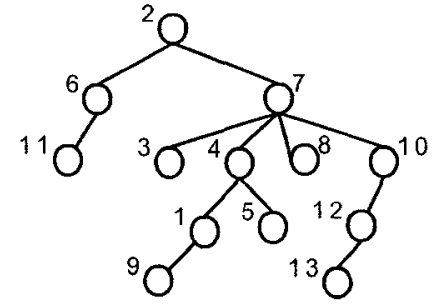

(a) Width $=50,000$

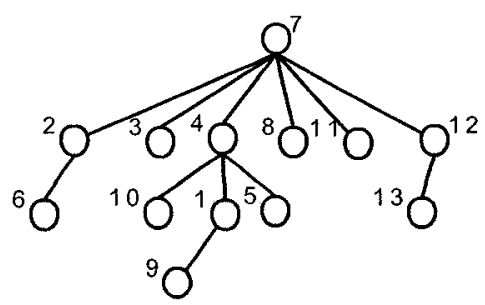

(b) Width $=100,000$

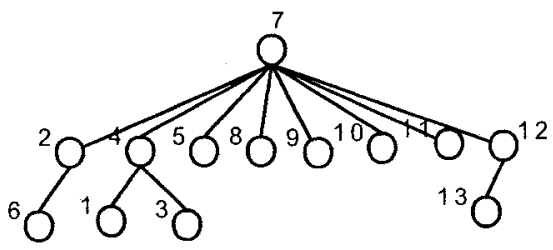

(c) Width $=150,000$

Figure 6.9: The best dependence tree for the Tarragona data Set obtained by using the $E M I M$ metric with various values of the width parameter.

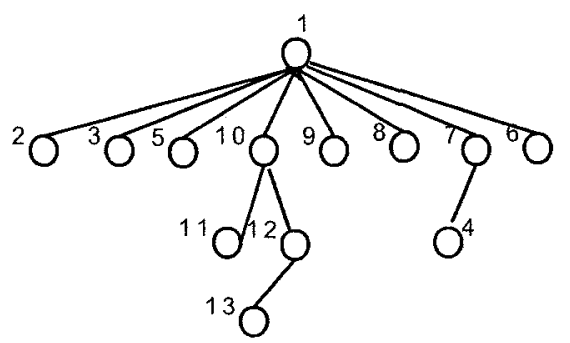

(a) Width $=1,000$

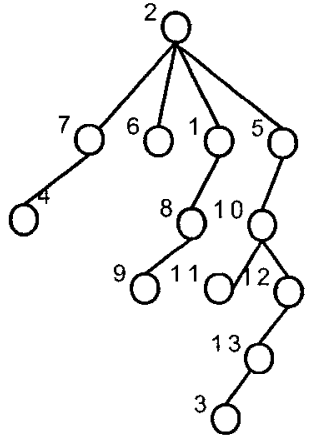

(b) Width $=5,000$

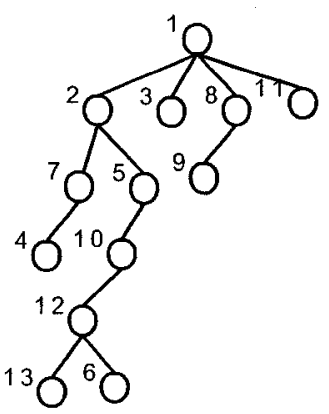

(c) Width $=10,000$

Figure 6.10: The best dependence tree for the Census data set obtained by using the $E M I M$ metric with various values of the width parameter. 
learning the dependence tree using the $M S T$ on the constructed fully-connected graph (where the edges weights are calculated using the EMIM or the $\chi^{2}$ metric) was able to converge to the true (unknown) dependence tree even if this sibling relationship was not known. The answer was always in the affirmative. By way of example, consider two binomial data sets with 6 variables. Both of them share the same dependency model between the variables, as shown in Figure 6.11. The only difference between these two data sets is that the values of the probabilities used to generate the random variables in the true tree structure - which in one case was sibling-related, and in the other was not sibling-related. Tables 6.2 and 6.3 show the values of the random probabilities which were used in generating each variable in the data set. Observe that in the first data set these values are related, while they are independent in the second data set. Figure show different snapshots of the convergence to the dependence model as the number of samples is increased.

The actual trees learnt for the data sets, as the number of samples processed increased, are given in Figures 6.12 and 6.13 respectively (reported at snapshots 50, 150 and 5,000). The decrease in the $E M I M$ and $c h i^{2}$ metrics with time are plotted in Figure 6.14. Observe that the final inferred tree in both cases is exactly the unknown tree - which, again, was correctly inferred, and that the values of both the metrics ultimately converged to the lowest possible values. Thus we conclude that the relationship between the probabilities of generation of the sibling random variables, was not able to "confuse" the algorithm in learning the unknown structure.

It should be mentioned, though, that in the cases in which the sibling probabilities were related, the learning was faster - which we believe is quite remarkable. All these figures assure that although we have exactly identical dependence model, the random probability values between the variables in addition of having dependent or independent probabilities between the sibling variables play predominant role in determining the rate of convergence and the number of records which are used to converge to the real model. 
Table 6.2: The probability values used in generating the corresponding random variables when the corresponding probabilities for the sibling nodes in the structural dependence tree are related.

\begin{tabular}{|c|c|}
\hline Probability & value \\
\hline \hline Prob(x1=0) & 0.40 \\
$\operatorname{Prob}(\mathrm{x} 1=1)$ & 0.60 \\
$\operatorname{Prob}(\mathrm{x} 2=0 \mid \mathrm{x} 1=0)$ & 0.30 \\
$\operatorname{Prob}(\mathrm{x} 2=0 \mid \mathrm{x} 1=1)$ & 0.10 \\
$\operatorname{Prob}(\mathrm{x} 3=0 \mid \mathrm{x} 1=0)$ & 0.70 \\
$\operatorname{Prob}(\mathrm{x} 3=0 \mid \mathrm{x} 1=1)$ & 0.90 \\
$\operatorname{Prob}(\mathrm{x} 4=0 \mid \mathrm{x} 1=0)$ & 0.20 \\
$\operatorname{Prob}(\mathrm{x} 4=0 \mid \mathrm{x} 1=1)$ & 0.60 \\
$\operatorname{Prob}(\mathrm{x} 5=0 \mid \mathrm{x} 3=0)$ & 0.80 \\
$\operatorname{Prob}(\mathrm{x} 5=0 \mid \mathrm{x} 3=1)$ & 0.40 \\
$\operatorname{Prob}(\mathrm{x} 6=0 \mid \times 3=0)$ & 0.15 \\
$\operatorname{Prob}(\mathrm{x} 6=0 \mid \mathrm{x} 3=1)$ & 0.76 \\
\hline
\end{tabular}

Table 6.3: The probability values used in generating the corresponding random variables when the corresponding probabilities for the sibling nodes in the structural dependence tree are unrelated.

\begin{tabular}{|c|c|}
\hline Probability & value \\
\hline \hline Prob $(\mathrm{x} 1=0)$ & 0.40 \\
Prob $(\mathrm{x} 1=1)$ & 0.60 \\
$\operatorname{Prob}(\mathrm{x} 2=0 \mid \mathrm{x} 1=0)$ & 0.30 \\
$\operatorname{Prob}(\mathrm{x} 2=0 \mid \mathrm{x} 1=1)$ & 0.10 \\
$\operatorname{Prob}(\mathrm{x} 3=0 \mid \mathrm{x} 1=0)$ & 0.60 \\
$\operatorname{Prob}(\mathrm{x} 3=0 \mid \mathrm{x} 1=1)$ & 0.70 \\
$\operatorname{Prob}(\mathrm{x} 4=0 \mid \mathrm{x} 1=0)$ & 0.20 \\
Prob $(\mathrm{x} 4=0 \mid \mathrm{x} 1=1)$ & 0.60 \\
$\operatorname{Prob}(\mathrm{x} 5=0 \mid \mathrm{x} 3=0)$ & 0.40 \\
$\operatorname{Prob}(\mathrm{x} 5=0 \mid \mathrm{x} 3=1)$ & 0.50 \\
$\operatorname{Prob}(\mathrm{x} 6=0 \mid \mathrm{x} 3=0)$ & 0.15 \\
$\operatorname{Prob}(\mathrm{x} 6=0 \mid \mathrm{x} 3=1)$ & 0.76 \\
\hline
\end{tabular}




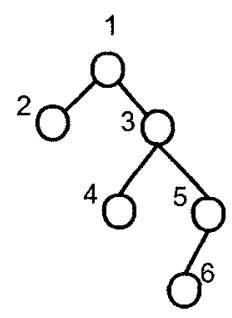

Figure 6.11: The best dependence tree for a binomial data sets with 5,000 records and 6 variables.

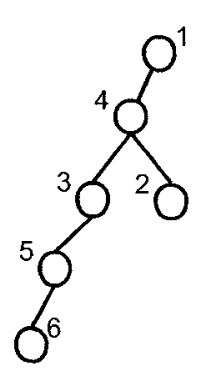

(a) $N=50$

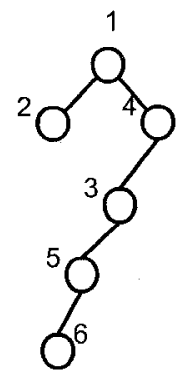

(b) $N=70$

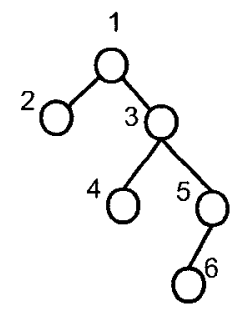

(c) $N=80$

Figure 6.12: The "inferred" dependence tree for the binary data set as the number of samples increases. In this case, the probabilities between the sibling random variables are related.

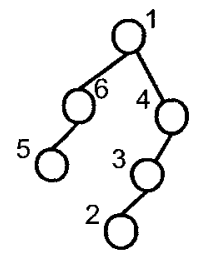

(a) $N=50$

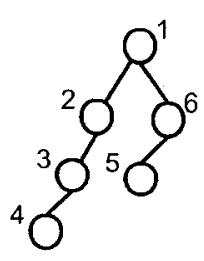

(b) $N=100$

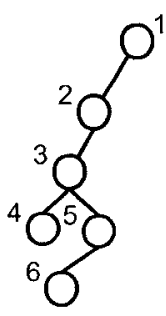

(c) $N=250$

Figure 6.13: The "inferred" dependence tree for the binary data set as the number of samples increases. In this case, the probabilities between the sibling random variables are unrelated. 


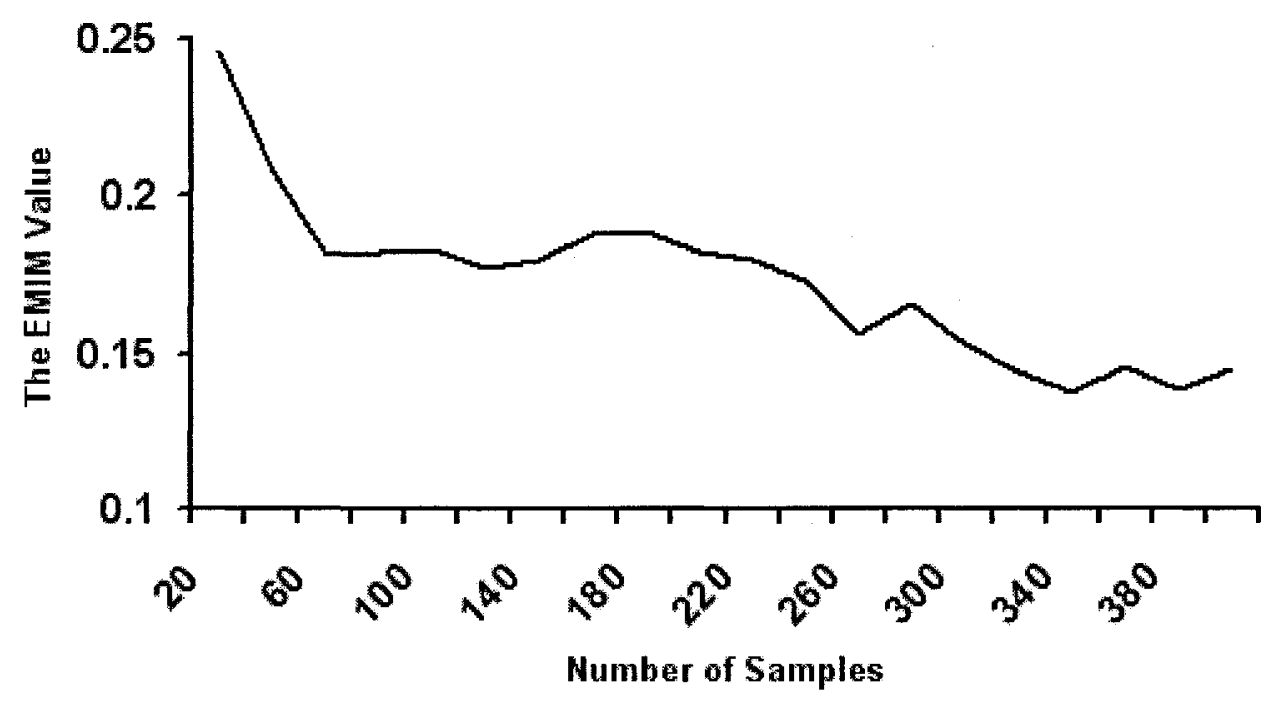

(a)

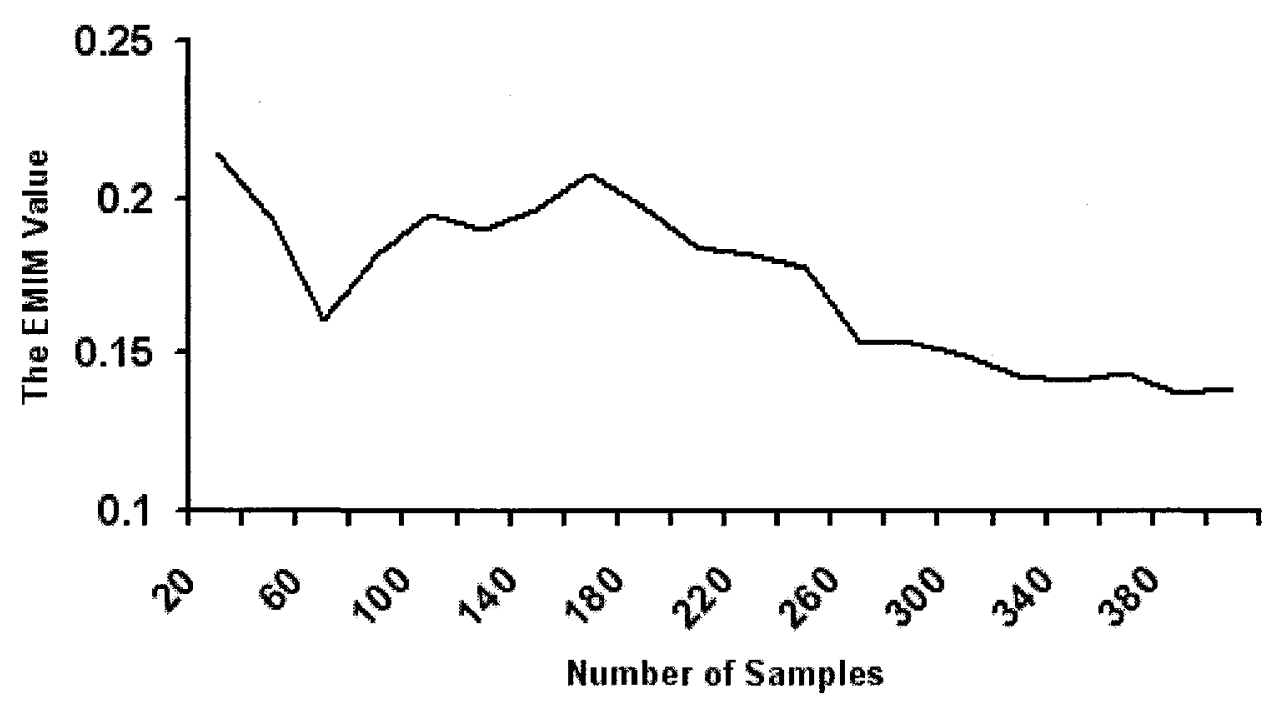

(b)

Figure 6.14: The convergence of the corresponding metric for the Set-Up4 data sets by using (a) the EMIM metric to calculate the edges weights. In (a) the probabilities between the siblings are related, and in (b) these probabilities between the siblings are unrelated. 


\section{- Experiment Sets 3:}

The third set of experiments verified our conjecture that it was expedient to use the sub-set of the variables obtained (from the best dependence tree) by projecting the micro-data file into 3,4 or 5 variables before invoking the multivariate micro-aggregation process.

Since an $M A T$ seeks to reduce the loss in the data utility, it must be pointed out here that the value of the $I L$ depends on the sub-set of variables used to microaggregate the multi-variate data file. As mentioned earlier, to infer the best sub-set of variables to be used in the micro-aggregation, we have to go through all the different projection possibilities. The results (Table 6.4) show that the estimation of the percentage value of the $I L$ for various data sets obtained by projecting the entire data set into specified number of variables prior to invoking the $M D A V$ method. The value of the $I L$ was bounded between the minimum value (in the fourth column) that was obtained by using the variable indices addressed in the third column, and the maximum value (in the sixth column) that was obtained by using the indices addressed in the fifth column. The last column in Table 6.4 represents the average value of the $I L$ over all the different combinations of projected variables in the micro-data file.

The most interesting observation was that the minimum value of the $I L$ obtained by using 3,4 or 5 projected variables in the Tarragona and Census data sets were exactly the same. This implies using the same "most independent variables", which in turn, preserve the same high value for the variance. Therefore, in the case of real-life data sets, we recommend projecting the entire micro-data file using 3 variables, since using a larger number of variables to project the micro-data file requires more time without leading to significant reduction in the $I L$ value.

Practically, due to the exponential number of combinations, we could not cover the entire solution space so as to reach to the best sub-set of the variables to be used in the micro-aggregation ${ }^{3}$. As opposed to this, by involving only

\footnotetext{
${ }^{3}$ On our processor, it took up to a few hours or even days depending on the dimensionality and
} 
Table 6.4: The value of the $I L$ obtained by using the $M D A V$ multi-variate $M A T$ after projecting various data sets into the specific number of variables.

\begin{tabular}{|c|c|c|c|c|c|c|c|}
\hline Data Set & $\begin{array}{c}\text { No. of } \\
\text { projected } \\
\text { variables }\end{array}$ & $\begin{array}{c}\text { No. of } \\
\text { possible } \\
\text { combinations }\end{array}$ & $\begin{array}{l}\text { Indices of the } \\
\text { variables used to } \\
\text { obtain the min. } \\
\text { value of } I L\end{array}$ & $\begin{array}{l}\text { The min. } \\
\text { value of } I L\end{array}$ & $\begin{array}{c}\text { Indices of the } \\
\text { variables used to } \\
\text { obtain the max. } \\
\text { value of } I L\end{array}$ & $\begin{array}{l}\text { The max. } \\
\text { value of } I L\end{array}$ & $\begin{array}{l}\text { The average } \\
\text { value of } I L\end{array}$ \\
\hline \multirow{5}{*}{ Tarragona } & 1 & 13 & 10 & $\overline{37.6374}$ & $\overline{8}$ & 48.1006 & 43.1017 \\
\hline & 2 & 78 & 11,13 & 24.7415 & 5,8 & 45.6925 & 31.6609 \\
\hline & 3 & 286 & $2,3,10$ & 20.7141 & $5,6,11$ & 34.1569 & 25.1587 \\
\hline & 4 & 715 & $2,3,10,11$ & 20.7141 & $5,6,11,12$ & 34.1569 & 25.4997 \\
\hline & 5 & 1287 & $2,3,10,11,12$ & 20.7141 & $5,6,11,12,13$ & 34.1569 & 25.6141 \\
\hline \multirow{5}{*}{ Census } & 1 & $\overline{13}$ & 10 & 38.2133 & 1 & 62.9093 & 45.79787 \\
\hline & 2 & 78 & 4,13 & 22.5795 & 1,8 & 55.634 & 31.618 \\
\hline & 3 & 286 & $7,8,10$ & 15.6043 & $1,8,9$ & 45.815 & 21.2046 \\
\hline & 4 & 715 & $7,8,10,11$ & 15.6043 & $1,8,9,10$ & 45.815 & 22.0308 \\
\hline & 5 & 1287 & $7,8,10,11,12$ & 15.6043 & $1,8,9,10,11$ & 45.815 & 22.8299 \\
\hline \multirow{5}{*}{ Sim_1 } & 1 & 8 & 2 & 56.6216 & 3 & 59.8342 & 58.6944 \\
\hline & 2 & 28 & 1,8 & 46.8576 & 3,5 & 51.3179 & 49.4823 \\
\hline & 3 & 56 & $2,4,7$ & 37.6486 & $4,6,7$ & 42.2961 & 39.7095 \\
\hline & 4 & 70 & $2,4,7,8$ & 37.6486 & $4,6,7,8$ & 42.2961 & 39.5901 \\
\hline & 5 & 56 & $1,3,5,6,7$ & 37.6522 & $3,4,6,7,8$ & 42.1577 & 39.7102 \\
\hline \multirow{4}{*}{$S i m \_2$} & 1 & 16 & 2 & 61.6118 & 11 & 63.0976 & 62.7308 \\
\hline & 2 & 120 & 1,16 & 56.6698 & 9,13 & 59.0037 & 58.2026 \\
\hline & 3 & 560 & $1,8,11$ & 51.6551 & $6,8,9$ & 54.3367 & 53.3249 \\
\hline & 4 & 1820 & $1,8,11,12$ & 51.6551 & $6,8,9,10$ & 54.3367 & 53.18 \\
\hline \multirow{3}{*}{$S i m \_3$} & 1 & 22 & 2 & 62.5849 & 7 & 64.0993 & 63.7251 \\
\hline & 2 & 231 & 2,22 & 59.0211 & 8,11 & 60.9375 & 60.4842 \\
\hline & 3 & 1540 & $2,7,13$ & 55.5274 & $4,6,14$ & 57.7998 & 56.9827 \\
\hline
\end{tabular}


the vertices that have the maximum number of $I / O$ edges in the connected undirected graph to micro-aggregate the micro-data file, we were able to obtain an acceptable value of the $I L$ close to its lower bound, and which is always (in all the cases) superior to the average value. Thus, such an automated strategy for projecting the multi-variate data sets will reduce the solution space to be searched which, in turn, reduces the computation time required to test the candidate variables, and to choose the best sub-set from them.

Tables 6.5 and 6.6 shows the percentage value of the $I L$ obtained by using our strategy in projecting the micro-data file into sub-sets of sizes 3 and 4 , respectively, prior to invoking the $M D A V$ method. When the Census data set was projected onto a number of variables prior to the micro-aggregation, the minimum values of the $I L$ were equal to $17.47 \%$ when the width value was equal to 1,000 and the number of variables was set to 3 or 4 , to $16.23 \%$ when the width value was equal to 5,000 and the number of variables was equal to 3 or 4 . The value of the minimum $I L$ was equal to $18.29 \%$ and to $17.70 \%$ when the width value was equal to 10,000 and the projection was onto 3 and 4 variables, respectively. It is worth mentioning that the values obtained were quite close to the lower bound of the $I L$, i.e., 15.60\%, as shown in Table 6.4, besides being superior to the average values over all the different combinations (i.e., $21.20 \%$ and $22.03 \%$ for 3 and 4 variables, respectively). Similar results were obtained for the Tarragona data set when the minimum value of the $I L$ using 3 or 4 variables was equal to $24.13 \%$ by setting the width value to 50,000 or 100,000 . But, it was equal to $25.05 \%$ when the width was 150,000 . Again, these values were closer to the lower bound of the $I L$ which was $20.71 \%$, and were superior to the average value which was close $25.5 \%$. In Tarragona data set, the minimum values of the $I L$, when the width value was set to 50,000 , 100,000 and 150,000 , were equal to $24.13 \%, 24.13 \%$ and $25.04 \%$, respectively. The values obtained were quite close to the lower bound of the $I L$, i.e., $20.71 \%$, as shown in Table 6.4, besides being superior to the average values over all the different combinations (i.e., $25.16 \%$ ). Finally, we would like to state that the 
simulated data set yielded similar results to those of the real data sets where the minimum values of the $I L$ were equal to $38.11 \%$ for $\operatorname{Sim} 1,51.95 \%$ for $\operatorname{Sim} 2$ and $55.82 \%$ for Sim3. These values were quite close to the lower bound of the $I L$ which were equal to $37.64 \%$ for $\operatorname{Sim} 1,51.65 \%$ for $\operatorname{Sim} 2$ and $55.52 \%$ for $\operatorname{Sim} 3$, respectively.

Table 6.5: The value of the $I L$ obtained by using the $M D A V$ multi-variate $M A T$ after projecting various data sets using 3 variables by using the $E M I M$ metric to calculate the edge weights in the connected undirected graph.

\begin{tabular}{|c|c|c|c|c|}
\hline Data set & Width value & No. of possibilities & Variable indices & $I L$ \\
\hline \multirow{10}{*}{ Tarragona } & \multirow{5}{*}{50,000} & \multirow{5}{*}{5} & $7,4,1$ & 24.1333 \\
\hline & & & $7,4,10$ & 24.1881 \\
\hline & & & $7,4,2$ & 25.0465 \\
\hline & & & $7,4,12$ & 25.6574 \\
\hline & & & $7,4,6$ & 25.6826 \\
\hline & \multirow{3}{*}{100,000} & \multirow{3}{*}{3} & $7,4,1$ & 24.1333 \\
\hline & & & $7,4,2$ & 25.0465 \\
\hline & & & $7,4,12$ & 25.6574 \\
\hline & & & $7,4,2$ & 25.0465 \\
\hline & 150,000 & 2 & $7,4,12$ & 25.6574 \\
\hline \multirow{9}{*}{ Census } & & & $1,10,7$ & 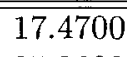 \\
\hline & 1,000 & 2 & $1,10,12$ & 25.3632 \\
\hline & \multirow{6}{*}{5,000} & \multirow{6}{*}{6} & $2,10,8$ & 16.2332 \\
\hline & & & $2,10,5$ & 17.3421 \\
\hline & & & $2,10,1$ & 17.7012 \\
\hline & & & $2,10,13$ & 21.0694 \\
\hline & & & $2,10,7$ & 21.1128 \\
\hline & & & $2,10,12$ & 21.5828 \\
\hline & 10,000 & 1 & $1,2,12$ & 18.2996 \\
\hline \multirow[b]{2}{*}{ Sim_1 } & \multirow[b]{2}{*}{100} & \multirow[b]{2}{*}{2} & $\overline{1,3,4}$ & 51.9684 \\
\hline & & & $1,3,6$ & 52.1126 \\
\hline \multirow[b]{2}{*}{ Sim_2 } & \multirow[b]{2}{*}{100} & \multirow[b]{2}{*}{2} & $1,3,4$ & 51.9684 \\
\hline & & & $1,3,6$ & 52.1126 \\
\hline \multirow[b]{2}{*}{$\operatorname{Sim} 3$} & & \multirow[b]{2}{*}{2} & $1,3,5$ & 56.1318 \\
\hline & 100 & & $1,3,18$ & 55.8246 \\
\hline
\end{tabular}

\section{- Experiment Sets 4:}

The fourth set of experiments compares the EMIM and $\chi^{2}$ metrics in calculating the edge weights in the connected undirected graph. Generally speaking, 
Table 6.6: The value of the $I L$ obtained by using the $M D A V$ multi-variate $M A T$ after projecting various data sets using 4 variables by using the EMIM metric to calculate the edge weights in the connected undirected graph.

\begin{tabular}{|c|c|c|c|c|}
\hline Data set & Width value & No. of possibilities & Variable indices & $I L$ \\
\hline \multirow{14}{*}{ Tarragona } & \multirow{10}{*}{50,000} & \multirow{10}{*}{10} & $7,4,1,10$ & 24.1333 \\
\hline & & & $7,4,1,12$ & 24.1333 \\
\hline & & & $7,4,10,12$ & 24.1881 \\
\hline & & & $7,4,1,6$ & 24.2114 \\
\hline & & & $7,4,1,2$ & 24.9648 \\
\hline & & & $7,4,2,10$ & 25.0465 \\
\hline & & & $7,4,2,12$ & 25.0465 \\
\hline & & & $7,4,6,10$ & 25.6826 \\
\hline & & & $7,4,6,12$ & 25.6826 \\
\hline & & & $7,4,2,6$ & 26.0992 \\
\hline & \multirow{3}{*}{100,000} & \multirow{3}{*}{3} & $7,4,1,12$ & 24.1333 \\
\hline & & & $7,4,1,2$ & 24.9648 \\
\hline & & & $7,4,2,12$ & 25.0465 \\
\hline & 150,000 & 1 & $7,4,2,12$ & 25.0465 \\
\hline \multirow{20}{*}{ Census } & 1,000 & 1 & $1,10,7,12$ & 17.4700 \\
\hline & \multirow{15}{*}{5,000} & \multirow{15}{*}{15} & $2,10,8,12$ & 16.2322 \\
\hline & & & $2,10,8,13$ & 16.2322 \\
\hline & & & $2,10,5,8$ & 17.0012 \\
\hline & & & $2,10,7,8$ & 17.0012 \\
\hline & & & $2,10,5,12$ & 17.3421 \\
\hline & & & $2,10,5,13$ & 17.3421 \\
\hline & & & $2,10,1,12$ & 17.7012 \\
\hline & & & $2,10,1,13$ & 17.7012 \\
\hline & & & $2,10,1,5$ & 19.4846 \\
\hline & & & $2,10,7,12$ & 21.1128 \\
\hline & & & $2,10,7,13$ & 21.1128 \\
\hline & & & $2,10,12,13$ & 21.5828 \\
\hline & & & $2,10,1,8$ & 21.9116 \\
\hline & & & $2,10,7,5$ & 23.0105 \\
\hline & & & $2,10,1,7$ & 26.4757 \\
\hline & \multirow{4}{*}{10,000} & \multirow{4}{*}{4} & $1,2,12,10$ & 17.7012 \\
\hline & & & $1,2,12.5$ & 19.4846 \\
\hline & & & $1,2,12,8$ & 21.9116 \\
\hline & & & $1,2,12,7$ & 26.4757 \\
\hline Sim_1 & 100 & 1 & $1, \overline{4,4,6}$ & 38.1105 \\
\hline$\overline{\text { Sim_2 }}$ & 100 & 1 & $\overline{1,3,4,6}$ & 51.9684 \\
\hline Sim_3 & 100 & 1 & $1,3,5,18$ & 56.1318 \\
\hline
\end{tabular}


the $\chi^{2}$ is faster in leading to a convergence to the best dependence tree than the EMIM metric since it required a smaller number of observations or records to converge. It is worth mentioning, though, that both metrics converged to the same true structure of the dependence model for the simulated data sets by setting the value of the width parameter to 100 . The scenario is completely different for the real data sets, as seen in Figures 6.15 and 6.16 which display different structures for the best dependence tree for the Tarragona and Census data sets, respectively, using various values for the width parameter. Table 6.7 shows the value of the $I L$ obtained by invoking the $M D A V$ method after projecting various data sets into three variables by using the $\chi^{2}$ metric to calculate the edges weights in the connected undirected graph. In the simulated sets, the $\chi^{2}$ metric led to the same value of the $I L$ which was obtained by using the EMIM metric because they converged to the same dependence tree, implying that they used the same set of variables to micro-aggregate the micro-data file. As opposed to this, in the real data sets, the $\chi^{2}$ converged to a different "best" dependence tree compared to the one obtained by using the $E M I M$ metric, thus leading to a different value of the $I L$. In general, the value of the $I L$ obtained by using the $\chi^{2}$ metric was lower than the corresponding value obtained by using the EMIM metric for the Census data sets, but it was higher than the value obtained by using the EMIM metric in Tarragona data set. Table 6.7 shows that the values of the $I L$ for the Tarragona data set, when the width value was set to $50,000,100,000$ and 150,000 , were equal to $25.1 \%$, $24.8 \%$ and $25.7 \%$, respectively, and for the Census data set the minimum values of the $I L$ were equal to $17.47 \%$ when the width value was set to $1,000,16.23 \%$ when the width value was set to 5,000 , and to $18.16 \%$ when the width value was set to 10,000 . In general, the $\chi^{2}$-based solution space was superior to the EMIM-based solution.

\section{- Experiment Sets 5:}

The distribution of the average of a set of random variables tends to be Normal, even when the distribution of the individual random variable is decidedly 


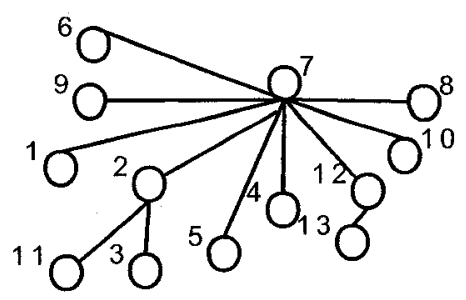

(a) Width $=50,000$

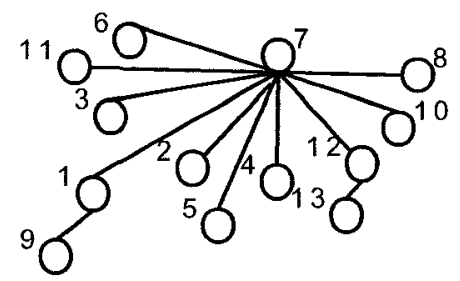

(b) Width $=100,000$

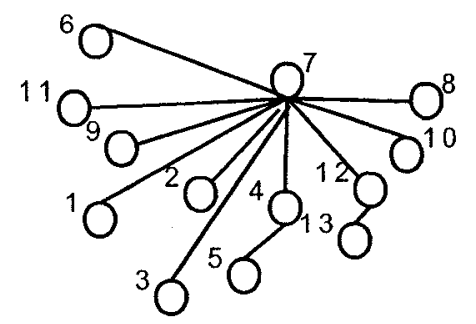

(c) Width $=150,000$

Figure 6.15: The best dependence tree for the Tarragona data set obtained by using the $\chi^{2}$ metric with various values of the width parameter.

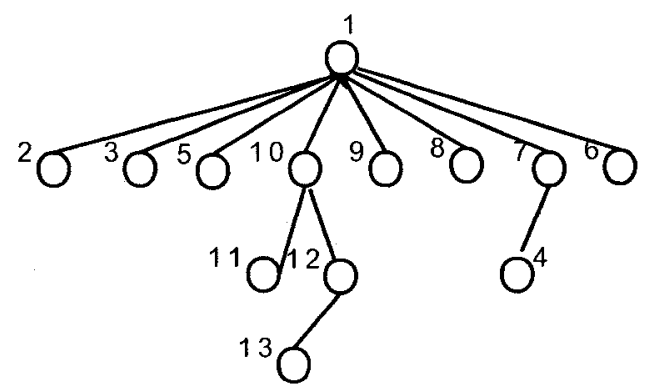

(a) Width $=1,000$

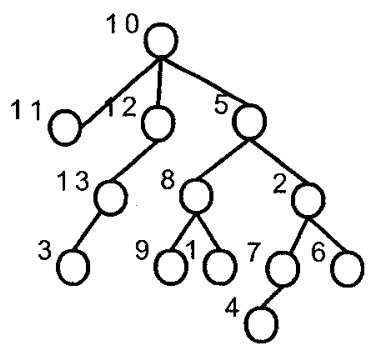

(b) Width $=5,000$

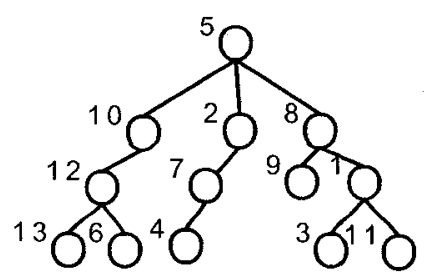

(c) Width $=10,000$

Figure 6.16: The best dependence tree for the Census data set obtained by using the $\chi^{2}$ metric with various values of the width parameter. 
Table 6.7: The value of the $I L$ obtained by using the $M D A V$ multi-variate $M A T$ after projecting various data sets using 3 variables by using the $\chi^{2}$ metric to calculate the edge weights in the connected undirected graph.

\begin{tabular}{|c|c|c|c|c|}
\hline Data set & Width value & No. of possibilities & Variable indices & $I L$ \\
\hline \multirow{3}{*}{ Tarragona } & 50,000 & 1 & $7,2,12$ & 25.0923 \\
\hline & 100,000 & 1 & $7,1,12$ & 24.8137 \\
\hline & 150,000 & 1 & $7,4,12$ & 25.6574 \\
\hline \multirow{9}{*}{ Census } & 1000 & $?$ & $1,10,7$ & 17.4700 \\
\hline & \multirow{4}{*}{5,000} & \multirow{4}{*}{4} & $\frac{\frac{1,10,12}{10,2,8}}{10,8}$ & 16.2322 \\
\hline & & & $5,8,2$ & 17.0012 \\
\hline & & & $10,5,2$ & 17.3421 \\
\hline & & & $10,5,8$ & 22.5430 \\
\hline & \multirow{4}{*}{10,000} & \multirow{4}{*}{4} & $1,5,8$ & 18.1627 \\
\hline & & & $1,8,12$ & 18.8725 \\
\hline & & & $1,5,12$ & 20.2778 \\
\hline & & & $5,8,12$ & 21.9324 \\
\hline \multirow[b]{2}{*}{ Sim_1 } & \multirow[b]{2}{*}{100} & \multirow[b]{2}{*}{2} & $1,3,4$ & 51.9684 \\
\hline & & & $1,3,6$ & 52.1126 \\
\hline \multirow[b]{2}{*}{ Sim_2 } & \multirow[b]{2}{*}{100} & \multirow[b]{2}{*}{2} & $\overline{11,3,4}$ & 51.9684 \\
\hline & & & $1,3,6$ & 52.1126 \\
\hline \multirow[b]{2}{*}{ Sim_3 } & \multirow[b]{2}{*}{100} & \multirow[b]{2}{*}{2} & $1,3,5$ & 56.1318 \\
\hline & & & $1,3,18$ & 55.8246 \\
\hline
\end{tabular}


non-Normal. This is a consequence of the Central Limit Theorem, which is the foundation for many statistical procedures, because the distribution of the phenomenon under study does not necessarily have to be Normal. Therefore, the last set of experiments assumes the Normality of the micro-data file to quickly compute the first and second order marginals, and to thus lead to the MST for computing the best dependence tree. Subsequently, we applied our strategy to choose the subset of random variables to project the file before invoking the $M D A V$ method.

Under an assumption of normality, the edge weight, $w_{i j}$, between two variables $V_{i}$ and $V_{j}$ in the connected undirected graph can be calculated by [136]:

$$
w_{i j}=-\frac{1}{2} \log \left(1-\rho_{i j}^{2}\right)
$$

where $\rho_{i j}^{2}$ represents the correlation coefficient between the two variables $V_{i}$ and $V_{j}$ in the graph.

The beauty of estimating the dependence model assuming normality is that it does not depend on any parametric value. Therefore, it leads to a unique $M S T$ if the edges weight are unique. Figure 6.17 shows the best dependence tree for the simulated and real data sets. It is worth mentioning that using the correlation between two random variables in calculating the edges weights of the graph does not lead to convergence to the "true" underlying dependence model in the case of the simulated data sets. However, generally the overall process yielded a value of $I L$ close to the minimum value of the $I L$ after projecting the entire data set into 3 variables although the search space was greater than the search space that resulted from using the $\chi^{2}$ or the EMIM metrics. The minimum value of the $I L$ was equal to $23.10 \%$ for Tarragona data set, $16.34 \%$ for Census data set, $37.8 \%$ for Sim $1,51.96 \%$ for Sim2, and $55.64 \%$ for Sim 3 .

Finally, we conclude by stating that each method of calculating the edges weights has its own advantages and disadvantages. We believe that, in practice, the user is the only one who is capable of deciding which is the most suitable metric for the specific 
Table 6.8: The value of the $I L$ obtained by using the $M D A V$ multi-variate $M A T$ after projecting various data sets into 3 variables assuming normality.

\begin{tabular}{|c|c|c|c|}
\hline Data set & No. of possibilities & Variable indices & $I L$ \\
\hline \multirow{5}{*}{ Tarragona } & \multirow{5}{*}{5} & $2,4,10$ & 23.1068 \\
\hline & & $2,4,1$ & 24.9648 \\
\hline & & $2,4,7$ & 25.0465 \\
\hline & & $2,4,12$ & 25.9818 \\
\hline & & $2,4,6$ & 26.0992 \\
\hline \multirow{5}{*}{ Census } & \multirow{5}{*}{5} & $4,10,5$ & 16.3416 \\
\hline & & $4,10,9$ & 16.8352 \\
\hline & & $4,10,6$ & 19.6712 \\
\hline & & $4,10,7$ & 20.2959 \\
\hline & & $4,10,12$ & 20.9725 \\
\hline \multirow{6}{*}{ Sim_1 } & \multirow{6}{*}{6} & $\overline{7,2,3}$ & 37.8147 \\
\hline & & $7,1,3$ & 37.8676 \\
\hline & & $7,1,6$ & 38.1610 \\
\hline & & $7,2,6$ & 38.1704 \\
\hline & & $7,3,6$ & 41.4843 \\
\hline & & $7,1,2$ & 42.0634 \\
\hline \multirow{3}{*}{$S i m \_2$} & \multirow{3}{*}{3} & $\overline{4,1,3}$ & 51.9684 \\
\hline & & $4,1,12$ & 52.0159 \\
\hline & & $4,3,12$ & 53.9267 \\
\hline \multirow{10}{*}{ Sim_3 } & \multirow{10}{*}{10} & $20,1,5$ & $\overline{55.6419}$ \\
\hline & & $20,2,13$ & 55.6631 \\
\hline & & $20,2,5$ & 55.8020 \\
\hline & & $20,2,3$ & 55.8722 \\
\hline & & $20,1,3$ & 55.9010 \\
\hline & & $20,1,13$ & 55.9474 \\
\hline & & $20,5,13$ & 57.1943 \\
\hline & & $20,3,5$ & 57.3903 \\
\hline & & $20,3,13$ & 57.4192 \\
\hline & & $20,1,2$ & 57.4770 \\
\hline
\end{tabular}




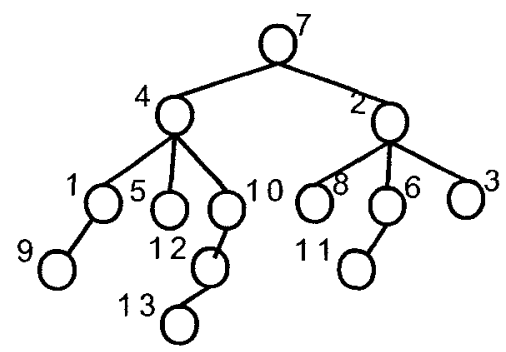

(a) Tarragona

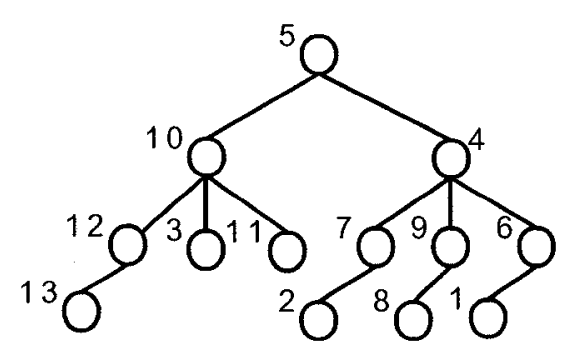

(b) Census

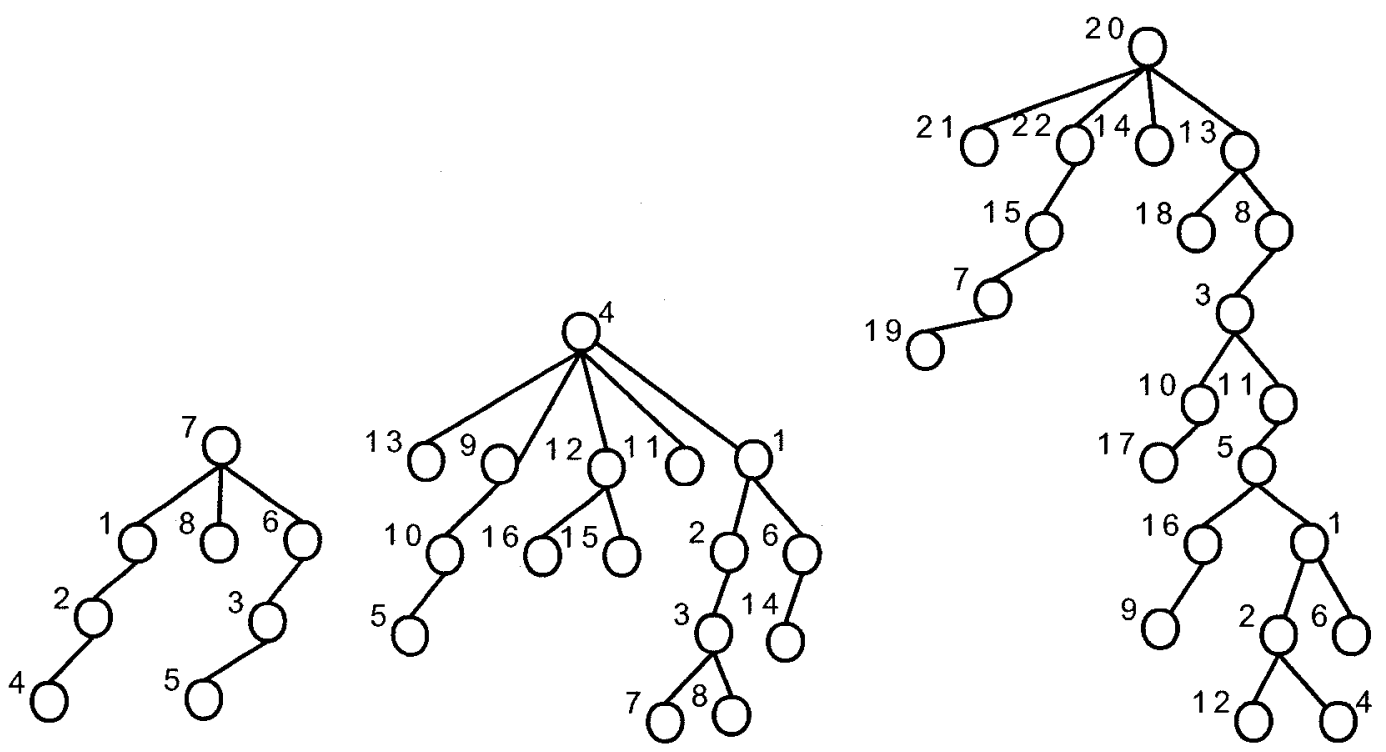

(c) Sim_1

(d) Sim_2

(e) Sim_3

Figure 6.17: The best dependence tree for the real and simulated data sets assuming normality. 
data sets. Table 6.9 summarizes the characteristics of each metric in calculating the edge weights of the connected undirected graph.

Table 6.9: Characteristics of the EMIM, $\chi^{2}$ and Correlation metrics in calculating the edges weights of the connected undirected graph.

\begin{tabular}{|c|c|c|c|}
\hline & $\overline{E M I M}$ & $\chi^{2}$ & Correlation \\
\hline Width parameter & Sensitive & Sensitive & Not sensitive \\
\hline $\begin{array}{l}\text { No. of Combinations } \\
\text { in search space }\end{array}$ & Medium & Small & Large \\
\hline $\begin{array}{l}\text { Convergence to the best } \\
\text { dependence tree structure }\end{array}$ & Converge & Converge & $\begin{array}{c}\text { Does not } \\
\text { always converge }\end{array}$ \\
\hline Convergence speed & $\begin{array}{c}\text { Slower than } \\
\chi^{2} \text { metric }\end{array}$ & $\begin{array}{c}\text { Slower than } \\
\text { assuming Normality }\end{array}$ & $\begin{array}{l}\text { Faster than } \\
\text { both metrics }\end{array}$ \\
\hline
\end{tabular}

\subsection{Conclusions}

In this chapter we have shown how the information about the structure of the dependence between the variables in the micro-data file can be used as a fundamental indicator before invoking any $M A T$. By using this information, we have proposed a new automated scheme as a pre-processing phase to determine the number and the identity of the variables that are to be used to micro-aggregate the micro-data file. This is achieved by constructing a connected undirected graph whose nodes represent the random variables in the micro-data file, edges represent the statistically dependencies, and the edges weights are computed either by using the EMIM, $\chi^{2}$ or the correlation values when a normality constraint is assumed. The experimental results show that such a methodology involving projecting the multi-variate data sets reduces the solution space, which further directly reduces the computation time required to search the entire space combinatorially. In spite of this, this methodology leads to a solution whose $I L$ values are close to the minimum value of the $I L$ that can be obtained by exhaustively searching over the entire search space. 


\section{Chapter 7}

\section{Conclusion and Future Research}

\subsection{Overall Contributions}

With the increasing ability of users to access data and information, privacy and security have become primary concerns. Simultaneously, the need to access detailed statistical information is becoming mandatory for various governmental and private companies. This Thesis dealt with the issue of security and the efficient utility of data in the protection of micro-data files in statistical agencies. We specifically considered the problem of preventing the disclosure of confidential information concerning particular individuals without significantly harming the utility of the data being protected. In this context, we argued that the $D R$ and $I L$, and a composite newly-devised metric are among the most important metrics for protecting the statistical data.

The research in this Doctoral Thesis focused on the family of $M A T s$ which include recent well-known techniques in the area of $S D C$ for micro-data. The rationale behind these techniques is the confidentiality rules of dissemination that allow publication if, and only if, each record in the published file corresponds to a group of a size equal to $k$ or more individuals, where no individual in the original file dominates the group excessively. In general, data aggregation is not only the central concept in 
data security and privacy, but also in many applications in computer science, such as artificial intelligence and data mining.

This section summarizes the work presented in this Thesis, and gives the overall conclusions of each chapter, and the lists of areas where further research can be conducted. In all brevity we mention that while Chapter 1 introduced the overall Thesis, and listed its main motivation and objectives, Chapter 2 embarked on a fairly comprehensive survey of the field of $S D C$ techniques in general and, more specifically, on the family of MATs. In addition to covering the different approaches to measure the $I L$ and $D R$, Chapter 2 described the existing methods available to resolve their conflicting goals.

The main contributions of the Thesis involve:

1. Enhancing the Heuristic $k$-Ward MAT.

2. Using Fixed Structure LA to Solve the MAP.

3. Using NN Methods to Solve the MAP.

4. Utilizing Dependence-Based Information for MATs.

We shall visit each of these in the next section.

\subsection{Enhancing the Heuristic $k$-Ward MAT}

\subsubsection{Contributions}

This chapter presented an enhancement to the $k$-ward MAT with respect to the required computational time. This was achieved by, first of all, minimizing the computational burden involved in evaluating the between-class distance matrix, and also by recursively partitioning the entire data set before invoking the standard $k$-Ward 
method. The latter optimization partitioned the data set using either a fixed threshold or a dynamic one. In this chapter we showed that invoking these two optimization strategies simultaneously could reduce the computational time up to $90 \%$. The various optimization strategies were applied to uni-variate data sets, and also on multi-variate data sets projected using the first principal component or the sum of $Z$-scores.

\subsubsection{Future work}

We foresee the following avenues for future research.

1. The question of investigating how these methods can be used for multi-variate online statistical databases is open.

2. Clearly, a shortest path algorithm will lead to an optimal solution. We submit that the philosophy that we have introduced in Chapter 3 is also valid for shortest-path-based algorithms. Indeed, the question of whether the shortest path for the graph can be approximately computed quickly using recursive invocations of shortest paths for its sub-graphs is a very interesting problem. Such a study remains open.

3. We believe that our recursive strategy can be rendered applicable to a "multivariate k-Ward" heuristic that does not rely on such a uni-variate projection. Although we are currently considering how our matrix optimizing operations can be applied to the scheme proposed in [45], it does not seem to be straightforward. This work is still open.

4. It is plausible that our proposed time-based optimization could induce some statistical bias in the way the data is aggregated. It would be interesting to see what impact this has with respect to the privacy issue. However, we add in passing, that such a bias-based analysis will be data and distribution dependent and, is thus, far from trivial. 
5. Problems related to statistical inference in databases have been solved with a variety of tools $[2,12,35,36,37,97,114]$. Incorporating these tools into our present framework would help to describe the limitations of our approach and/or the types of data sets that are, or are not, well suited for such an aggregation technique. Interestingly, this could involve the extension of our mechanism for discrete or ordinal data elements. All of these are open problems, warranting research "projects" in their own right!

\subsection{Using Fixed Structure LA to Solve the MAP}

\subsubsection{Contribution}

This chapter proposed the use of a sophisticated learning algorithm, namely the $O M M A$, to solve the $M A P$ and optimize the $M A T$. The method was based on the notion of specifying various levels of uncertainties for pairs of individuals belonging to the same group. The $O M M A$ increased or decreased these uncertainty levels depending on whether a known similar pair is "correctly" or "incorrectly" clustered by the algorithm. The new algorithm was compared extensively to another algorithm that was developed and implemented in the $\mu$-Argus software package $(M D A V)$. It yielded the same results as the latter for the uni-variate case, and showed improvement for multi-variate micro-aggregation. The improvement in the value of the $I L$ measure was about $10 \%$ on real data sets, and up to $13 \%$ on simulated data sets. Preliminary tests showed that the proposed strategy scales well with respect to the size of the data set, the dimensionality, and the group size. By defining a composite measure involving the $I L$ and $D R$, we proved that proposed strategy also obtains a minimum score value when compared to the $M D A V$ method. This indicates that the $O M M A$ technique is probably the best $M A T$ not only from the $I L$ perspective, but also from the viewpoint of a measure which was a combination of the $I L$ and $D R$. 


\subsubsection{Future Work}

We foresee the following avenues for future research.

1. It will be interesting to see how we can extend the $O M M A$ for the data-oriented micro-aggregation, where the group size, $n_{i}$, satisfies $k \leq n_{i}<2 k$.

2. The question of studying whether the minimum group size constraint can be treated as a "soft constraint" associated with a certain preference level is open.

3. The problem of incorporating other families of learning automata, especially those of continuous variable structure types, with a fixed-size or variable-size group constraints, is also unsolved.

4. The question of applying the concepts of hierarchical $O M M A$ for a large data set is also an interesting avenue for future work.

5. Finally, it would be good if some studies were conducted to compare the $O M M A$ to the $M D A V$ with respect to the computational time for large real data sets, typically containing more than 100,000 records.

\subsection{Using NN Methods to Solve the MAP.}

\subsubsection{Contribution}

This chapter provided a practical methodology (for micro-aggregating the micro-data file) that is based on the theory of $N N s$ and their novel clustering properties. Our newly-proposed scheme, referred to as the $I A M A T$, introduced the Association and the Interaction between records as a new metric for measuring the similarity. The $I A M A T$ strategy preserved the meaning of the closeness between the records so as to coalesce them into groups, and to also maximize the actual Interaction among the records inside each group. In general, incorporating the concepts of the Interaction 
among the records and their mutual Association, had been shown to be advantageous to solving the $M A P$, because they have the effect of minimizing the $I L$. Our experiments clearly showed a significant improvement to the state-of-the-art $M D A V$. The improvement in the value of the $I L$ measure was about $8 \%$ on real data sets and up to $14 \%$ on simulated data sets. This indicated that the resulting clusteres results in better grouping compared to the classical Euclidean distance approach. Preliminary tests showed that the new strategy scales well with respect to the cardinality of the data set, the dimensionality, and the group size. Again, the method also yields a superior $S I$ value when compared to the $M D A V$ method. This indicated that the $I A M A T$ is probably one of the best MATs not only from the $I L$ perspective, but also from the viewpoint of a measure which is a combination of the $I L$ and $D R$.

\subsubsection{Future Work}

We foresee two avenues for future research.

1. It would be fascinating to study how we could extend the IAMAT towards data-oriented $M A T$, where the group size, $n_{i}$, satisfies $k \leq n_{i}<2 k$.

2. The question of investigating the effect of having a dynamic value of $\alpha$ on the compactness of each group and on the value of the $I L$ remains unsolved. We believe that we can compute the dynamic value of $\alpha$ in any iteration based on the remaining unassigned records at the end of every iteration in the clustering phase. As opposed to this, the fixed value is computed once before invoking the clustering phase, and is based on the entire data set. 


\subsection{Utilizing Dependence-Based Information for MATs}

\subsubsection{Contribution}

This chapter discussed how the presence and structure of dependency between a set of random variables in the micro-data file is valuable information that can be used as a fundamental indicator before invoking any $M A T$. We proposed a new automated scheme as a pre-processing phase to determine the number and the identity of the variables that are to be used in the micro-aggregation phase. This is achieved by constructing a completely connected, undirected graph whose nodes represent the random variables in the micro-data file, whose edges represent the statistically dependencies, and whose edge weights are computed by using either an information theoretic, $\chi^{2}$ or correlation-based measure. The advantageous and disadvantages of these respective measures were investigated. The experimental results showed that such a methodology involving projecting the multi-variate data sets reduces the solution space, which further directly reduces the computation time required to search the entire space combinatorially. Additionally, this methodology leads to a solution whose $I L$ values were close to the minimum value of the $I L$ obtained by exhaustively searching over the entire search space. In general, this chapter presented an entirely new approach to tackling a tough problem for which no prior solution was available.

\subsubsection{Future Work}

We foresee three avenues for future research.

1. We believe that our newly automated scheme can be rendered applicable to any known $M A T$. Therefore, it will be interesting to see how it can be applied to the $O M M A$, and the $I A M A T$.

2. An investigation on the effect of this methodology on the value of the $D R$ would also be interesting. 
3. As argued above, the use of dependence information can help to reduce the required computational time in the aggregation stage, so as to effectively measure the distance between the records. We believe that capturing such dependence information is not only important to $M A T s$, but also to other $S D C$ techniques, such as data swapping. However, since data swapping annihilates multi-variate relationships - such as the regression and the correlations between two or more variables [96] - the question of considering how the swapping can be used on a selected set of dependent variables without disturbing or removing any mutual relationships, remains open.

\subsection{Future Research Avenues}

From the literature we have reviewed, it appears as if there is a scarcity of good practical methods for MATs which are based on perturbative techniques. We do not imply that there are no methods available, or that none of them perform well. On the contrary, as mentioned in Chapter 2, numerous methods have been proposed and studied, especially in the recent past, where novel schemes for specific applications have been developed. But based on the fact that this field is relatively new, none of these methods have attained to such a degree of maturity so as to grant it the right to be called a "standard benchmark technique" worthy to be imbedded as a built-in function and labeled as being the "optimal" strategy. However, some of the available methods are very promising, and, generally speaking, using an adaptive clustering method in an MAT represents a very positive approach. This is because it will reduce the area of the search space significantly by enforcing the group size constraint $[15,79,130,138]$. Additionally, combining data mining tools with the $M A T$ can be very beneficial since a resulting scheme can yield a good trade-off between the $D R$ and the $I L$ criteria $[59,131,137]$.

Several overall research directions for further investigation arise from this Thesis. They are discussed below. 
- The need to further merge the areas of $L A$ and micro-aggregation so as to devise a novel $M A T$, which can provide variable-size groups for a multi-variate data set, is open. We know that the main challenge in micro-aggregation is to design good heuristics which minimize the $I L$ and the $D R$. This can be achieved by providing a variable-group size which, in turn, implies a lower loss in the data utility because it generates more homogeneous groups. This, of-course, also leads to the preservation of the natural data aggregation by allowing the group sizes to be between $k$ and $2 k-1$. In addition, a variable-group size will result in decreasing the likelihood of the information being compromised. However, such a variable-group size constraint would directly imply a more complex implementation than the schemes which constrain a fixed-size, as explained in Chapter 4.

The primary disadvantage of the current $O M M A$ scheme is that the microdata file is to be equi- partitioned. With a little insight it is not trivial to translate the fixed- size constraint into the variable-size constraint. Hence, this improvement will decrease the $I L$ and the $D R$. Generalizing the $O M M A$ to handle the variable group size constraint will increase the difficulty of the problem, since we have no formal method of determining how to characterize the optimal solution. The main concept utilized in the $O M M A$ version was the limit imposed on the number of records in each group. The purpose of setting this limit was to prevent having any interleaving groups. But if variable-group size is used, this limit is no longer valid since each class may or may not have exactly the same number of groups. In other words, in the current version we assumed a knowledge of the number of the groups. In contrast, in a future study, we would seek a solution, which requires the number of groups to be bounded between $n /(2 k-1)$ and $n / k$.

Due to the fact that each group may have a different number of records (i.e., between $k$ and $2 k-1$ ), the religious invocation of the $O M M A$ is not applicable. With a little insight, it is easy to see that the scenario encountered for a reward does not pose any explicit problem (see Section 4.3). This is because enforcing 
the reward condition does not alter the basic constitution of the groups. On the other hand, the problems encountered on enforcing a penalty are numerous which deserve further investigation.

- Numerous government organizations, such as Statistics Canada, aim to release uscful statistical summaries for public use and for analysis, without violating the confidentiality requirements. An extremely fascinating avenue for future work is to focus on applying our newly-developed techniques to such real-life data sets. The main goal of such studies will be to test the applicability of the applied methodology in a real scenario, and to assess their ability to overcome the relevant limitations and handicaps of the currently-used methods. Besides this, it would be beneficial to analyze the effect of the new micro-aggregation schemes from the perspectives of regression, dependency, and linear models. Such an investigation would also attempt to extensively study the effect of the micro-aggregation process on the $I L, D R$, and the $S I$.

- The wavelet transformation possesses the characteristic of providing spatial and frequency domain information, which play a predominant rule in many applications such as image compression, watermarking, and clustering. An integration between the fields involving wavelet transformation and micro-aggregation would prove to be extremely rewarding. To the best of our knowledge, we are not aware of any MAT that utilizes the characteristics of wavelets during the micro-aggregation process. We believe that such a transformation can guide the process of micro-aggregation by operating in the transformed feature space, and by thus identifying clusters at different levels of the transformed space. The goal of such a research endeavor would be to understand how this integration can be achieved while simultaneously preserving almost the same computational time and minimizing the loss in the data utility and the risk of disclosing the confidentiality.

- The processes of updating, deleting, or inserting individual records in the microdata file causes a serious problem in the problem of micro-aggregation. This, in turn, implies a large burden on the time and storage required to re-compute 
the micro-aggregated file after the change. The question of devising MATs in the presence of time varying data files remain open, and would definitely lead to many future research projects and theses. 


\section{Bibliography}

[1] M. Adachi and K. Aihara. Associative Dynamics in a Chaotic Neural Network. Neural Networks, 10(1):83-98, 1997.

[2] N. Adam and J. Wortmann. Security-Control Methods for Statistical Databases: A Comparative Study. ACM Computing Surveys, 21(4):515-556, 1989.

[3] M. Agache and B. Oommen. Generalized Pursuit Learning Schemes: New Families of Continuous and Discretized Learning Automata. IEEE Transactions on Systems, Man, and Cybernetics, Part B, 32(6):738-749, 2002.

[4] C. Aggarwal and P. Yu. Privacy-Preserving Data Mining. Springer, 2008.

[5] C. Aggarwal and P. Yu. Privacy-Preserving Data Mining: Models and Algorithms (Advances in Database Systems). Springer Science and Business Media L.L.C., 2008.

[6] R. Agrawal, T. Imielinski, and A. Swami. Mining Association Rules Between Sets of Items in Large Databases. In In Proccedings of ACM SIGMOD, pages 207-216, USA: Washington DC, 1993.

[7] R. Agrawal, H. Mannila, H. Srikant, R. Toivonen, and I. Verkamo. Fast Discovery of Association Rules. In Advances in Knowledge Discovery and Data Mining, pages 307-328, 1996.

[8] N. Anwar. Micro-Aggregation - The Small Aggregates Method. Technical report, Luxembourg: Eurostat, 1993. 
[9] F .Bacao, V .Lobo, and M. Painho. Self-organizing Maps as Substitutes for K-Means Clustering. In International Conference on Computational Science, pages 476-483, 2005.

[10] Y. Baeyens and D. Defays. Estimation of Variance Loss Following Microaggregation by the Individual Ranking Method. In Proceedings of Statistical Data Protection'98, pages 101-108, Luxembourg: Office for Official Publications of the European Communities, 1999.

[11] L. Bechet and P. Loosveldt. Development of Micro-aggregation Software. Technical report, Ariane II,275 route d'Arlon, L-8011 Strassen, Luxembourg, 2000.

[12] L. Beck. A Security Mechanism for Statistical Database. ACM Trans. Database Syst., 5(3):316-3338, 1980.

[13] R. Brand. Microdata Protection through Noise Addition. In Inference Control in Statistical Databases, From Theory to Practice, pages 97-116, London, UK, 2002. Springer-Verlag.

[14] R. Brand, J. Domingo-Ferrer, and J. Mateo-Sanz. Reference Data Sets to Test and Compare SDC Methods for Protection of Numerical Microdata. Technical report, CASC PROJECT, Computational Aspects of Statistical Confidentiality, 2002.

[15] P. Brucker. On the Complexity of Clustering Problems. In R. Hehn, B. Korte, and W. Oettli, editors, Optimization and Operations Research, pages 45-54. Berlin: Springer-Verlag, 1977.

[16] S. Castano, M. Fugini, G. Martella, and P. Samarati. Database security. Addison-Wesley, 1994.

[17] C. Chow and C. Liu. Approximating Discrete Probability Distributions with Dependence Trees. IEEE Trans. Information Theory, 14(11):462-467, 1968.

[18] T. Cormen, C. Leiserson, and R. Rivest. Introduction to Algorithms. MIT Press, McGraw-Hill, 1990. 
[19] G. Crises. Additive Noise for Microdata Privacy Protection in Statistical Databases. Technical report, 2004.

[20] G. Crises. An Introduction to Microdata Protection for Database Privacy. Technical report, 2004.

[21] G. Crises. Information Loss Measures for Microdata in Database Privacy Protection. Technical report, 2004.

[22] G. Crises. Microaggregation for Privacy Protection in Statistical Databases. Technical report, 2004.

[23] G. Crises. Microdata Disclosure Risk in Database Privacy Protection. Technical report, 2004.

[24] G. Crises. Non-Perturbative Methods for Microdata Privacy in Statistical Databases. Technical report, 2004.

[25] G. Crises. Perturbative Masking for Microdata Privacy Protection in Statistical Databases. Technical report, 2004.

[26] G. Crises. Synthetic Microdata Generation for Database Privacy Protection. Technical report, 2004.

[27] G. Crises. Trading off Information Loss and Disclosure Risk in Database Privacy Protection. Technical report, 2004.

[28] M. Cuppen. Secure Data Perturbation in Statistical Disclosure Control. PhD thesis, Statistics Netherlands, 2000.

[29] R. Dandekar, M. Cohen, and N. Kirkendall. Sensitive Micro Data Protection Using Latin Hypercube Sampling Technique. In Inference Control in Statistical Databases, From Theory to Practice, pages 117-125, London, UK, 2002. Springer-Verlag.

[30] C. Date. An Introduction to Database Systems. Addison-Wesely, 2000. 
[31] D. Defays. Protecting Microdata by Microaggregation: the Experience in Eurostat. Questiio, 21:221-231, 1997.

[32] D. Defays and M. Anwar. Masking Micro-data Using Micro-Aggregation. Journal of Official Statistics, 14(4):449-461, 1998.

[33] D. Defays and N. Anwar. Micro-Aggregation: A Generic Method. In Proceedings of the 2nd International Symposium on Statistical Confidentiality, pages 69-78, Luxembourg: Office for Official Publications of the European Communities, 1995.

[34] D. Defays and P. Nanopoulos. Panels of Enterprises and Confidentiality: the Small Aggregates Method. In Proceedings of 92 Symposium on Design and Analysis of Longitudinal Surveys, pages 195-204, Ottawa: Statistics Canada, 1993.

[35] D. Denning. Secure Statistical Databases with Random Sample Queries. ACM Trans. Database Syst., 5(3):291-315, 1980.

[36] D. Denning and P. Denning. Data Security. ACM Comput. Surv., 11(3):227249,1979 .

[37] D. Denning and P. Denning. The Tracker: A Threat to Statistical Database Security. ACM Trans. Database Syst., 4(1):76-96, 1979.

[38] J. Domingo-Ferrer. Statistical Disclosure Control in Catalonia and the CRISES Group. Technical report, 1999.

[39] J. Domingo-Ferrer. Microaggregation: Achieving k-Anonymity with QuasiOptimal Data Quality. In Proceedings of Q2006: European Conference on Qulity in Survey Statistics, 2006.

[40] J. Domingo-Ferrer. Microaggregation for Database and Location Privacy. volume 4032, pages 106-116, 2006. 
[41] J. Domingo-Ferrer. Microaggregation for Database and Location Privacy. In Next Generation Information Technologies and Systems-NGITS'2006, pages $106-116,2006$.

[42] J. Domingo-Ferrer, A. Martínez-Ballesté, J. Mateo-Sanz, and F. Sebé. Efficient Multivariate Data-Oriented Micro-Aggregation. The International Journal on Very Large DataBases, (VLDB), 15(4):355-369, Sep. 2006.

[43] J. Domingo-Ferrer and J. Mateo-Sanz. On Resampling for Statistical Confidentiality in Contingency Tables. Computers and Mathematics with Applications, 38:13-32, 1999.

[44] J. Domingo-Ferrer and J. Mateo-Sanz. An Empirical Comparison of SDC Methods for Continuous Micro-data in Terms of Information Loss and Disclosure Risk. In Joint ECE/Eurostat Work Session on Statistical Data Confidntiality, Conference of European Satisticians, Statistical Commision and Economic Commision for Europe, 2001.

[45] J. Domingo-Ferrer and J. Matco-Sanz. Practical Data-Oriented Microaggregation for Statistical Disclosure Control. IEEE Transactions on Knowledge and Data Engineering, 14(1):189-201, 2002.

[46] J. Domingo-Ferrer, J. Mateo-Sanz, A. Oganian, V. Torra, and A. Torres. On The Security of Microaggregation with Individual Ranking: Analytical Attacks. International Journal of Uncertainty, Fuzziness and Knowledge-Based Systems, 10(5):477-491, 2002.

[47] J. Domingo-Ferrer, J. Mateo-Sanz, and F. Sebe. Information Loss in Continuous Hybrid Microdata: Subdomain-Level Probabilistic Measures. In Soft Computing for Information Retrieval on the Web: Models and Applications, Studies in Fuzziness, pages 287-298, 2006.

[48] J. Domingo-Ferrer, J. Mateo-Sanz, and V. Torra. Comparing SDC Methods for Microdata on the Basis of Information Loss and Disclosure Risk. In Proc. of 
ETK-NTTSŠ2001, volume 2, pages 807-825, Luxembourg: Office for Official Publications of the European Communities, 2001.

[49] J. Domingo-Ferrer and F. Sebé. Optimal multivariate 2-microaggregation for microdata protection: a 2-approximation. In Privacy Statistical Databases, pages 129-138, Italy: Rome, 2006.

[50] J. Domingo-Ferrer, F. Sebé, and J. Castellà-Roca. On the Security of Noise Addition for Privacy in Statistical Databases. pages 149-161, Spain: Barcelona, 2004. Berlin: Springer-Verlag.

[51] J. Domingo-Ferrer, F. Sebe, and A. Solanas. A Polynomial-Time Approximation to Optimal Multivariate Microaggregation. Computers and Mathematics with Applications, 55:714-732, 2008.

[52] J. Domingo-Ferrer, A. Solanas, and A. Martínez-Balleste. Privacy in Statistical Databases: k-Anonymity Through Microaggregation. In Proceedings of IEEE Granular Computing 2006, pages 774-777, 2006.

[53] J. Domingo-Ferrer and V. Torra. A Quantitative Comparison of Disclosure Control Methods for Microdata. In P. Doyle, J. Lane, J. Theeuwes, and L. Zayatz, editors, Confidentiality, Disclosure and Data Access: Theory and Practical Applications for Statistical Agencies, pages 113-134, Amesterdam: North-Holland, 2002. Berlin: Springer-Verlag.

[54] J. Domingo-Ferrer and V. Torra. Aggregation Techniques for Statistical confidentiality. In Aggregation operators: new trends and applications, pages 260271, Germany: Heidelberg, 2002. Physica-Verlag GmbH.

[55] J. Domingo-Ferrer and V. Torra. Disclosure Control Methods and Information Loss for Microdata. In Confidentiality, Disclosure and Data Access: Theory and Practical Applications for Statistical Agencies, pages 93-112, Amsterdam: North-Holland, 2002. 
[56] J. Domingo-Ferrer and V. Torra. Extending Microaggregation Procedures Using Defuzzification Methods for Categorical Variables. In IEEE Intelligent Systems'2002, pages 44-49, Bulgaria: Varna, 2002.

[57] J. Domingo-Ferrer and V. Torra. Fuzzy Microaggregation for Microdata Protection. Journal of Advanced Computational Intelligence and Intelligent Informatics (JACIII), 7(2):153-159, 2003.

[58] J. Domingo-Ferrer and V. Torra. Median-Based aggregation operators for Prototype Construction in Ordinal Scales. International Journal of Intelligent Systems, 18(6):633-655, 2003.

[59] J. Domingo-Ferrer and V. Torra. On the Connections Between Statistical Disclosure Control for Microdata and Some Artificial Intelligence Tools. Information Sciences, 151:153-170, 2003.

[60] J. Domingo-Ferrer and V. Torra. Ordinal, Continuous and Heterogeneous kAnonymity Through Microaggregation. Data Mining and Knowledge Discovery, 11(2):195-212, 2005.

[61] R. Duda, P. Hart, and D. Stork. Pattern Classification. A Wiley-Interscience Publication, 2000.

[62] A. El-Hamdouchi and P. Willet. Hierarchic Document Clustering Using Ward's Mcthod. In In proceedings of the Ninth Annual International ACM SIGIR Conference on Research and Development in Information Retrieval, pages 149$156,1986$.

[63] M. Elliot. Integrating File and Record Level Disclosure Risk Assessment. In Inference Control in Statistical Databases, From Theory to Practice, pages 126134, London, UK, 2002. Springer-Verlag.

[64] E. Fayyoumi and B. Oommen. A Fixed Structure Learning Automaton MicroAggregation Technique for Secure Statistical Databases. In Privacy Statistical Databases, pages 114-128, Italy: Rome, 2006. 
[65] E. Fayyoumi and B. Oommen. On Optimizing the $k$-Ward Micro-Aggregation Technique for Secure Statistical Databases. In 11th Austratasian Conference on Information Security and Privacy Proceeding, pages 324-335, Australia: Melbourne, 2006.

[66] E. Feige and H. Watts. Protection of Privacy Through Microaggregation. In Data bases, Computers and the Social Sciences, pages 261-272. Wiley, 1970.

[67] F. Felso, J. Theeuwes, and G. Wagner. Disclosure Limitation Methods in Use: Results of A Survey. In Confidentiality, Disclosure and Data Access, pages 17-42, Amsterdam: North-Holland, 2001.

[68] L. Feng, T. Dillon, H. Weigana, and E. Chang. An XML-Enabled Association Rule Framework. In In Proc. of DEXA'03, pages 88-97, Prague: Czech Republic, 2003.

[69] E. Fernandez, R. Summers, and C. Wood. Databases Security and Integrity. Addison-Wesley, 1980.

[70] W. Fuller. Masking Procedures for Microdata Disclosure Limitation. Journal of Official Statistics, 9(2):383-406, 1993.

[71] W. Gale, S. Das, and C. Yu. Improvements to an Algorithm for Equipartitioning. IEEE Trans. Comput., 39(5):706-710, 1990.

[72] S. Giessing and A. Hundepool. The CASC Project: Integrating Best Practice Methods for Statistical Confidentiality. Technical report, 2003.

[73] J. Gouweleeuw, P. Kooiman, L. Willenborg, and P. Wolf. Post Randomization for Statistical Disclosure Control: Theory and Implementation. Technical report, 1997.

[74] S. Hansen and S. Mukherjee. A Polynomial Algorithm for Univariate Optimal Microaggregation. IEEE Transactions on Knowledge and Data Engineering, 15(4):1043-1044, 2003. 
[75] G. Horn and B. Oommen. A Fixed-Structure Learning Automaton Solution to the Stochastic Static Mapping Problem. In 19th IEEE International Parallel and Distributed Processing Symposium (IPDPS'05), pages 297-304, 2005.

[76] A. Hundepool, J. Domingo-Ferrer, L. Franconi, S. Giessing, R. Lenz, J. Longhurst, E. Nordholt, G. Seri, and P. Wolf. Handbook on Statistical Disclosure Control. a CENtre of EXcellence for Statistical Disclosure Control CENEX SDC, 2006.

[77] A. Hundepool, A. Wetering, R. Ramaswamy, L. Franconi, A. Capobianchi, P. Wolf, J. Domingo-Ferrer, V. Torra, R. Brand, and S. Giessing. M-ARGUS Version 4.0 Software and User's Manual, 2004.

[78] A. Hundepool, L. Wetering, L. Gemerden, A. Wessels, M. Fischetti, J. Salazar, and A. Caprara. t-ARGUS User's Manual, 1998.

[79] A. Jain, M. Murty; and P. Flynn. Data Clustering: A Review. ACM Computing Surveys, 31(3):264-323, 1999.

[80] W. Jonge. Compromising statistical Databases Responding to Queries About Means. ACM Trans. Database Syst., 8(1):60-80, 1983.

[81] K. Kenthapadi. DIMACS Working Group on Challenges for Cryptographers in Health Data Privacy. Technical report, 2004.

[82] J. Kim and W. Winkler. Masking Microdata Files. In Proceedings of the Section on Survey Research Methods, pages 114-119, 1995.

[83] P. Kooiman, L. Willenborg, and J. Gouweleeuw. PRAM: A Method for Disclosure Limitation of Microdata. Technical report, 1998.

[84] M. Laszlo and S. Mukherjee. Minimum Spanning Tree Partitioning Algorithm for Microaggregation. IEEE Transactions on Knowledge and Data Engineering, 17(7):902-911, 2005. 
[85] Y. Li, S. Zhu, L. Wang, and S. Jajodia. A Privacy-Enhanced Microaggregation Method. In FoIKS '02: Proceedings of the Second International Symposium on Foundations of Information and Knowledge Systems, pages 148-159, London, UK, 2002. Springer-Verlag.

[86] S. Mandujano. Inference attacks to statistical databases: Data suppression, concealing controls and other security trends. Technical report, Purdue University, Department of Computer Science, 2001.

[87] M. Markey, J. Lo, G. Tourassi, and C. Floyd Jr. Self-Organizing Map for Cluster Analysis of A Breast Cancer Database. Artificial Intelligence in Medicine, 27:113-127, 2003.

[88] A. Martinez-Balleste, A. Solanas, J. Domingo-Ferrer, and J. Mateo-Sanz. A Genetic Approach to Multivariate Microaggregation for Database Privacy. In ICDE Workshops, pages 180-185, 2007.

[89] M. Mas. Statistical Data Protection Techniques. Technical report, Eustat: Euskal Estatistika Erakundea,Instituto Vasco De Estadistica, 2006.

[90] J. Mateo, J. Domingo-Ferrer, F. Sebé, A. Martinez-Balleste, A.Torres, and N.Macia. New Microaggregation Algorithms Software Documentation and Related Papers. Technical report, CASC PROJECT, Computational Aspects of Statistical Confidentiality, 2002.

[91] J. Mateo-Sanz and J. Domingo-Ferrer. A Comparative Study of Microaggregation Methods. Questiio, 22(3):511-526, 1998.

[92] J. Mateo-Sanz and J. Domingo-Ferrer. A Method for Data-Oriented Multivariate Microaggregation. In Proceedings of Statistical Data Protection'98, pages 89-99, Luxembourg: Office for Official Publications of the European Communities, 1999.

[93] J. Mateo-Sanz, J. Domingo-Ferrer, and F. Sebé. Probabilistic Information Loss Measures in Confidentiality Protection of Continuous Microdata. Data Mining and Knowledge Discovery, 11(2):181-193, 2005. 
[94] J. Mateo-Sanz, F. Sebé, and J. Domingo-Ferrer. Outlier Protection in Continuous Microdata Masking. pages 201-215, Spain: Barcelona, 2004. Berlin: Springer-Verlag.

[95] S. Misra and B. Oommen. Dynamic Algorithms for the Shortest Path Routing Problcm: Learning Automata-Based Solutions. IEEE Trans Syst Man Cybern B Cybern, 35(6):1179-1192, 2005.

[96] R. Moore. Controlled Data-Swapping Techniques for Masking Public Use Microdata Sets. Technical report, Statistical Research Division Report Series, Washington D. C., 1996.

[97] K. Muralidhar and R. Sarathy. Security of Random Data Perturbation Methods. ACM Trans. Database Syst., 24(4):487-493, 1999.

[98] K. Narendra and M. Thathachar. Learning Automata: An Introduction. New Jersey: Prenticc-Hall, 1989.

[99] K. Narendra, E. Wright, and L. Mason. Application of Learning Automata to Telephone Traffic Routing and Control. IEEE Trans Syst Man Cybern B Cybern, SMC-7(11):785-792, 1977.

[100] J. Nin and V. Torra. Modeling Projections in Microaggregation. In proceedings of the $12^{\text {th }}$ International Conference on Information Processing and Management of Uncertainty in Knowledge-Based System (IPMU), pages 138-145, 2008.

[101] E. Nordholt. Statistical Disclosure Control from A Users' Point of View. Technical report, Statistics Netherlands, 2001.

[102] A. Oganian. Security and Information Loss in Statistical Data Protection. PhD thesis, University URV Catalunya, 2002.

[103] A. Oganian and J. Domingo-Ferrer. On The Complexity of Optimal Microaggregation for Statistical Disclosure Control. Statistical Journal of the United Nations Economic Comission for Europe, 18(4):345-354, 2001. 
[104] A. Oganian and F. Karr. Combinations of SDC Methods for Microdata Protection. In Privacy Statistical Databases, pages 102-113, Italy: Rome, 2006.

[105] B. Oommen and S. Croix. String Taxonomy Using Learning Automata. IEEETSMC: IEEE Transactions on Systems, Man, and Cybernetics, 27(2):354$365,1997$.

[106] B. Oommen and E. Fayyoumi. An AI-Based Causal Strategy for Securing Statistical Databases Using Micro-Aggregation. In 21st Austratasian Joint Conference on Artificial Intelligence, To appear.

[107] B. Oommen and E. Fayyoumi. A Novel Method for Micro-Aggregation in Secure Statistical Databases Using Association and Interaction. In Information and Communications Security, 9th International Conference on Information and Communications Security, LNCS 4861, Springer Verlag, pages 126-140, 2007.

[108] B. Oommen and E. Fayyoumi. Enhancing Micro-Aggregation Technique by Utilizing Dependence-Based Information in Secure Statistical Databases. In Information Security and Privacy, 13 th Austratasian Conference on Information Security and Privacy Proceeding, LNCS 5107, Springer Verlag, pages 404-418, 2008.

[109] B. Oommen and C. Fothergill. Fast Learning Automaton-Based Image Examination and Retrieval. The Computer Journal, 36(6):542-553, 1993.

[110] B. Oommen and J. Lanctot. Discretized Pursuit Learning Automata. Systems, Man and Cybernetics, IEEE Transactions on, 20:931-938, 1990.

[111] B. Oommen and D. Ma. Fast Object Partitioning Using Stochastic Learning Automata. In SIGIR '87: Proceedings of the 10th annual international ACM SIGIR conference on Research and development in information retrieval, pages 111-122, New York, NY, USA, 1987. ACM Press.

[112] B. Oommen and D. Ma. Deterministic Learning Automata Solutions to the Equipartitioning Problem. IEEE Transaction Computer, 37(1):2-13, 1988. 
[113] B. Oommen and T. Roberts. Continuous Learning Automata Solutions to the Capacity Assignment Problem. IEEE Transactions on Computers, 49(6):608$620,2000$.

[114] M. Palley and J. Simonoff. The Use of Regression Methodology for the Compromise of Confidential Information in Statistical Databases. ACM Trans. Database Syst., 12(4):593-608, 1987.

[115] J. Panaretos and N. Tzyvidis. Aspects of Estimation Procedures at Eurostat with Some Emphasis on Over-Space Harmonization. In HERCMA 2001 CONFERENCE, 2001.

[116] J. Park, S. Chen, and P. Yu. Using A Hash-Based Method with Transaction Trimming for Mining Association Rules. IEEE Transactions on Knowledge and Data Engineering, 9:813-826, 1997.

[117] B. Reddy. Aspects of Database Security and Program Security. Technical report, Kanwal Rekhi School of Information Technology, Indian Institute of Technology-Bombay, 2003.

[118] S. Sahni. Data Structure, Algorithms, and Applications in C++. McGraw-Hill, 1998.

[119] J. Sanchez, J. Urrutia, and E. Ripoll. Test Report on Multivariate Microaggregation in m-Argus 3.2. Technical report, CASC PROJECT, Computational Aspects of Statistical Confidentiality, 2003.

[120] J. Sanchez, J. Urrutia, and E. Ripoll. Trade-Off between Disclosure Risk and Information Loss Using Multivariate Microaggregation: A Case Study on Business Data. In J. Domingo-Ferrer and V. Torra, editors, Privacy in Statistical Databases: CASC Project International Workshop, PSD 2004 Proceedings, pages 307-322, Spain: Barcelon, 2004. Berlin: Springer-Verlag.

[121] G. Sande. Methods for Data Directed Micro-aggregation in One Dimension. In Proceedings of NTTS and ETK: New Techniques and Technologies for Statistics and Exchange for Technology and Know-how, Crete: 18-22 June. 
[122] G. Sande. Exact and Approximate Methods for Data Directed Microaggregation in One or More Dimensions. Int. J. Uncertain. Fuzziness Knowl.-Based Syst., 10(5):459-476, 2002.

[123] J. Schatz. Survey of Techniques for Securing Statistical Databases. Technical report, University of California at Davis, 1997.

[124] F. Sebé, J. Domingo-Ferrer, J. Mateo-Sanz, and V. Torra. Post-Masking Optimization of the Tradeoff between Information Loss and Disclosure Risk in Masked Microdata Sets. In Inference Control in Statistical Databases, From Theory to Practice, pages 163-171, London, UK, 2002. Springer-Verlag.

[125] S. Skiena. The Algorithm Design Manual. Springer-Verlag, 1998.

[126] A. Solanas and A. Martínez-Ballestéé. V-MDAV: A Multivariate Microaggregation With Variable Group Size. In 17th COMPSTAT Symposium of the IASC, Rome, 2006.

[127] A. Solanas, A. Martiínez-Ballestéé, J. Domingo-Ferrer, and J. Mateo-Sanz. A 2d-Tree-Based Blocking Method for Microaggregating Very Large Data Sets. In The First International Conference on Availability, Reliability and Security. The International Dependability Conference Bridging Theory and Practice, 2006 .

[128] A. Solanas, A. Martinez-Balleste, J. Mateo-Sanz, and J. Domingo-Ferrer. Multivariate Microaggregation Based Genetic Algorithms. In 3rd International IEEE Conference on Intelligent Systems, pages 65--70, 2006.

[129] G. Sullivan and W. Fuller. The Use of Measurement Error To Avoid Disclosure. In The Section on Survey Research Methods, pages 802-807, 1989.

[130] D. Tasoulis and M. Vrahatis. Unsupervised Clustering on Dynamic Databases. Pattern Recognition Letters, 26:2116-2127, 2005. 
[131] V. Torra. Microaggregation for Categorical Variables: A Median Based Approach. In J. Domingo-Ferrer and V. Torra, editors, Privacy in Statistical Databases: CASC Project International Workshop, PSD 2004 Proceedings, pages 162-174, Spain: Barcelon, 2004. Berlin: Springer-Verlag.

[132] V. Torra, J. Abowd, and J. Domingo-Ferrer. Using mahalanobis distance-based record linkage for disclosure risk assessment. In Privacy in Statistical DatabasesPSD 2006, pages 233-242, 2006.

[133] V. Torra and J. Domingo-Ferrer. Towards Fuzzy C-Means Based Microaggregation. In P. Grzegorzewski, O. Hryniewicz, and M. Gil, editors, Advances in Soft Computing: Soft Methods in Probability, Statistics and Data Analysis, pages 289-294, Germany: Heidelberg, 2002. Physica-Verlag.

[134] V. Torra and S. Miyamoto. Evaluating Fuzzy Clustering Algorithms for Microdata Protection. In J. Domingo-Ferrer and V. Torra, editors, Privacy in Statistical Databases: CASC Project International Workshop, PSD 2004 Proceedings, pages 175-186, Spain: Barcelona, 2004. Berlin: Springer-Verlag.

[135] R. Valiveti and B. Oommen. On Using the Chi-Squared Metric for Determining Stochastic Dependence. Pattern Recognition, 25(11):1389-1400, 1992.

[136] R. Valiveti and B. Oommen. Determining Stochastic Dependence for Normally Distributed Vectors Using the Chi-squared Metric. Pattern Recognition, 26(6):975-987, 1993.

[137] A. Valls, V. Torra, and J. Domingo-Ferrer. Aggregation Methods to Evaluate Multiple Protected Versions of the Same Confidential Data Set. In P. Grzegorzewski, O. Hryniewicz, and M. Gil, editors, Soft Methods in Probability, Statistics and Data Analysis (Warsaw, 2002), pages 355-362, Germany: Heidelberg, 2002. Physica-Verlag.

[138] M. Vrahatis, B. Boutsinas, P. Alevizos, and G. Pavlides. The New k-Windows Algorithm for Improving the k-Means Clustering Algorithm. Journal of Complexity, 18:375-391, 2002. 
[139] J. Ward. Hierarchical Grouping to Optimize an Objective Function. J. American Statistical Association, 58:236-245, 1963.

[140] T. Wende. Different Grades of Statistical Disclosure Control Correlated with German Statistics Law. pages 336-342, Spain: Barcelona, 2004. Berlin: Springer-Verlag.

[141] L. Willenborg and T. Waal. Statistical Disclosure Control in Practice. Lecture Notes in Statistics 111, 1996.

[142] L. Willenborg and T. Waal. Elements of Statistical Disclosure Control. New York: Springer-Verlag, 2001. ILL Number: 2132712.

[143] R. Wilson and P. Rosen. Protecting Data through Perturbation Techniques: The Impact on Knowledge Discovery in Databases. Journal of Database Management, 14(2):14-26, 2003.

[144] W. Winkler. Re-identification Methods for Masked Microdata. In J. DomingoFerrer and V. Torra, editors, Privacy in Statistical Databases: CASC Project International Workshop, PSD 2004, pages 216-223, Spain: Barcelona, 2004. Berlin: Springer-Verlag.

[145] P. Wolf, J. Gouweleeuw, P. Kooiman, and L. Willenborg. Reflections on PRAM. Technical report, 1997.

[146] W. Yancey, W. Winkler, and R. Creecy. Disclosure Risk Assessment in Perturbative Microdata Protection. In Inference Control in Statistical Databases, From Theory to Practice, pages 135-152, London, UK, 2002. Springer-Verlag.

[147] Y. Yang, J. Carbonell, R. Brown, T. Pierce, B. Archibald, and X. Liu. Learning approaches for detecting and tracking news events. IEEE Intelligent Systems, $14(4): 32-43,1999$.

[148] Y. Yao, L. Chen, and Y. Chen. Associative Clustering for Clusters of Arbitrary Distribution Shapes. Neural Processing Letters, 14:169-177, 2001. 
[149] Y. Yao, L. Chen, A. Goh, and A.Wong. Clustering Gene Data via Associative Clustering Neural Network. In the 9th International Conference on Neural Information Processing (ICONIP'02), volume 5, pages 2228-2232, 2002.

[150] A. Zaslavsky and N. Horton. Balancing Disclosure Risk Against the Loss of Nonpublication. Journal of Official Statistics, pages 411-419, 1998. 